\begin{tabular}{|l|l|}
\hline $\begin{array}{l}\text { 2. To: (Receiving Organization) } \\
\text { Distribution }\end{array}$ & $\begin{array}{l}\text { 3. From: (Originating Organization) } \\
\text { Nuclear Safety and Licensing }\end{array}$ \\
\hline $\begin{array}{l}\text { 5. Proj./Prog./Dept./Div.: } \\
\text { Nuclear Safety and Licensing }\end{array}$ & $\begin{array}{l}\text { 6. Design Authority/Design Agent/Cog. Engr.: } \\
\text { D. R. Bratzel }\end{array}$ \\
\hline
\end{tabular}

8. Originator Remarks:

This document is being released into the supporting document system for retrievability purposes

11. Receiver Remarks:

For release

\section{A. Design Baseline Document? $\square$ Yes $\bigotimes$ No}

4. Related EDT No.:
N/A
7. Purchase Order No.:
N/A
9. Equip./Component No.:

$\mathrm{N} / \mathrm{A}$

10. System/Bldg./Facility:

N/A

12. Major Assm. Dwg. No.:

$N / A$

13. Permit/Permit Application No.:

$\mathrm{N} / \mathrm{A}$

14. Required Response Date

$N / A$

\begin{tabular}{|l|l|}
\hline \multicolumn{1}{|l|}{\begin{tabular}{|} 
(A) \\
Item \\
No.
\end{tabular}} & (B) Document/Drawing No. \\
\hline & RPP -6888 \\
\hline & \\
\hline & \\
\hline & \\
\hline & \\
\hline & \\
\hline
\end{tabular}

DATA TRANSMITTED

\begin{tabular}{|c|c|}
\hline $\begin{array}{c}\text { (C) Sheet } \\
\text { No. }\end{array}$ & $\begin{array}{c}\text { (D) Rev. } \\
\text { No. }\end{array}$ \\
\hline & 0 \\
\hline & \\
\hline & \\
\hline
\end{tabular}

16.

\begin{tabular}{|c|}
\hline Approval Designator (F) \\
\hline E, S, Q, D OR N/A \\
(See WHC-CM-3-5, \\
Sec. 12.7)
\end{tabular}

17.

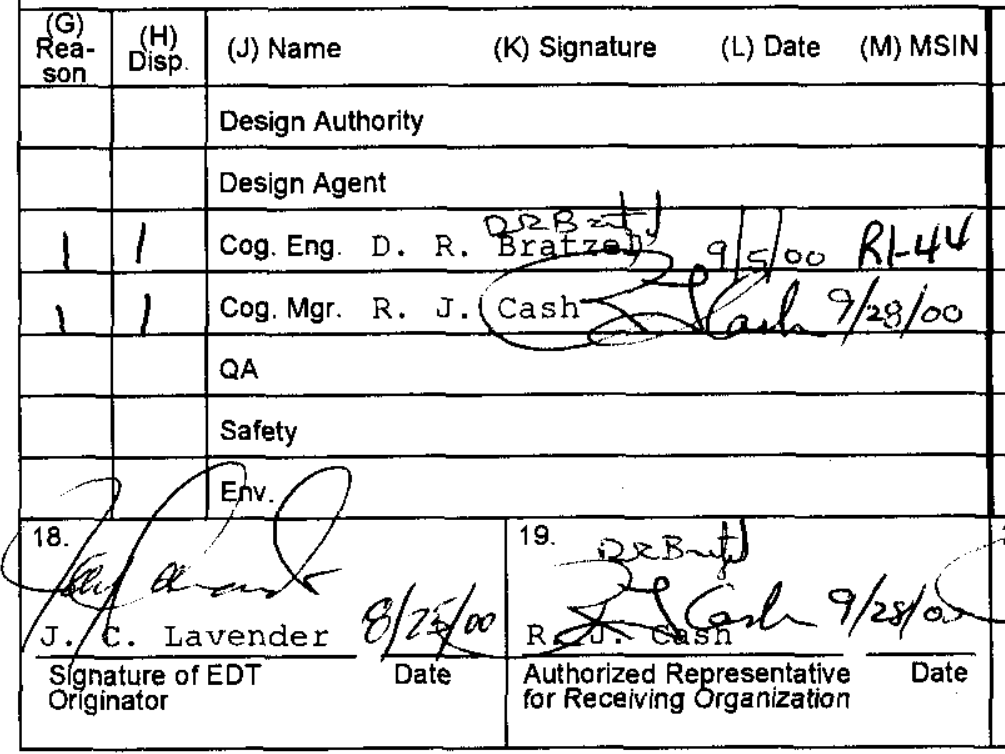

(E) Title or Description of Data Transmitted

RESOLVE! Version 2.5:

Flammable Gas Accident Test Plan and Test Results
Analysis Tool Acceptance

\begin{tabular}{|c|c|c|c|}
\hline$(F)$ & $(G)$ & $(H)$ & $(I)$ \\
\hline $\begin{array}{c}\text { Approval } \\
\text { Desig- } \\
\text { nator }\end{array}$ & $\begin{array}{c}\text { Reason } \\
\text { for Trans- } \\
\text { mittal }\end{array}$ & $\begin{array}{c}\text { Origi- } \\
\text { nator } \\
\text { Dispo- } \\
\text { stion }\end{array}$ & $\begin{array}{c}\text { Receiv- } \\
\text { er } \\
\text { Dispo- } \\
\text { sition }\end{array}$ \\
\hline N/A & 2 & 1 & 1 \\
\hline & & & \\
\hline & & & \\
\hline & & & \\
\hline
\end{tabular}

KEY
Reason for Transmittal (G)
6. Dist. (Receipt Acknow. Required)
4. Review
5. Post-Review

SIGNATURE/DISTRIBUTION

(See Approval Designator for required signatures)
Disposition (H) \& (I)

$\begin{array}{ll}\text { 1. Approved } & \text { 4. Reviewed no/comment } \\ \text { 2. Approved w/comment } & \text { 5. Reviewed w/comment } \\ \text { 3. Disapproved w/comment } & \text { 6. Receipt acknowledged }\end{array}$

(J)
(K) Signature

(L) Date

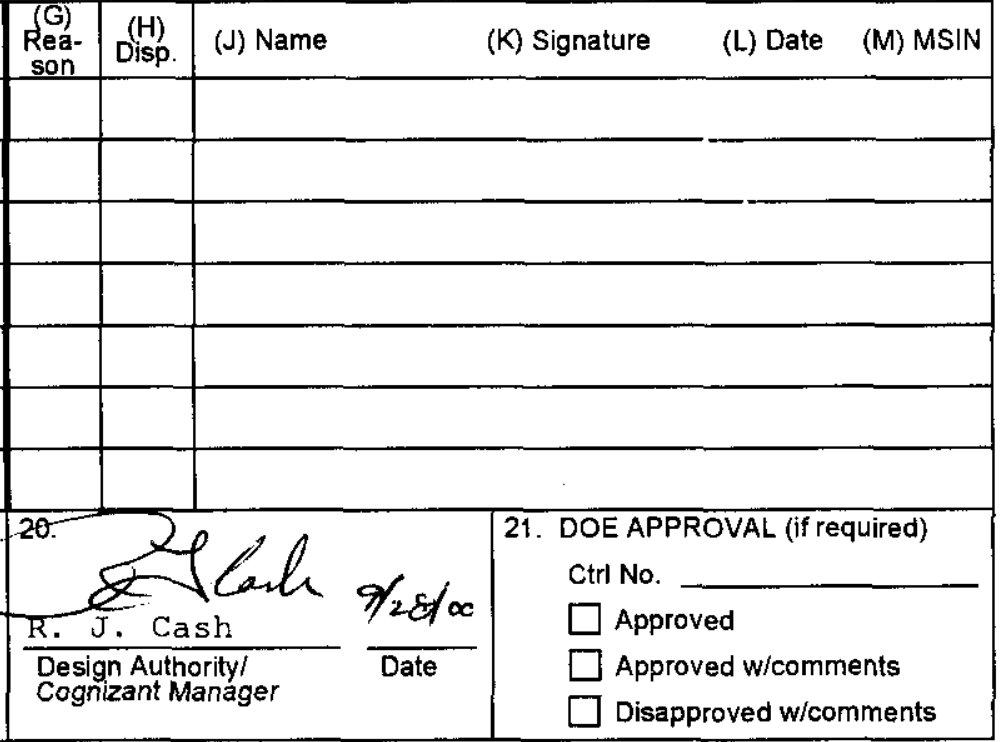

(M) MSIN 


\section{DISTRIBUTION SHEET}

To

Distribution

Project Title $N$ Work Order

RESOLVE! Version 2.5: Flammable Gas Accident Analysis Tool

Acceptance Test Plan and Test Results

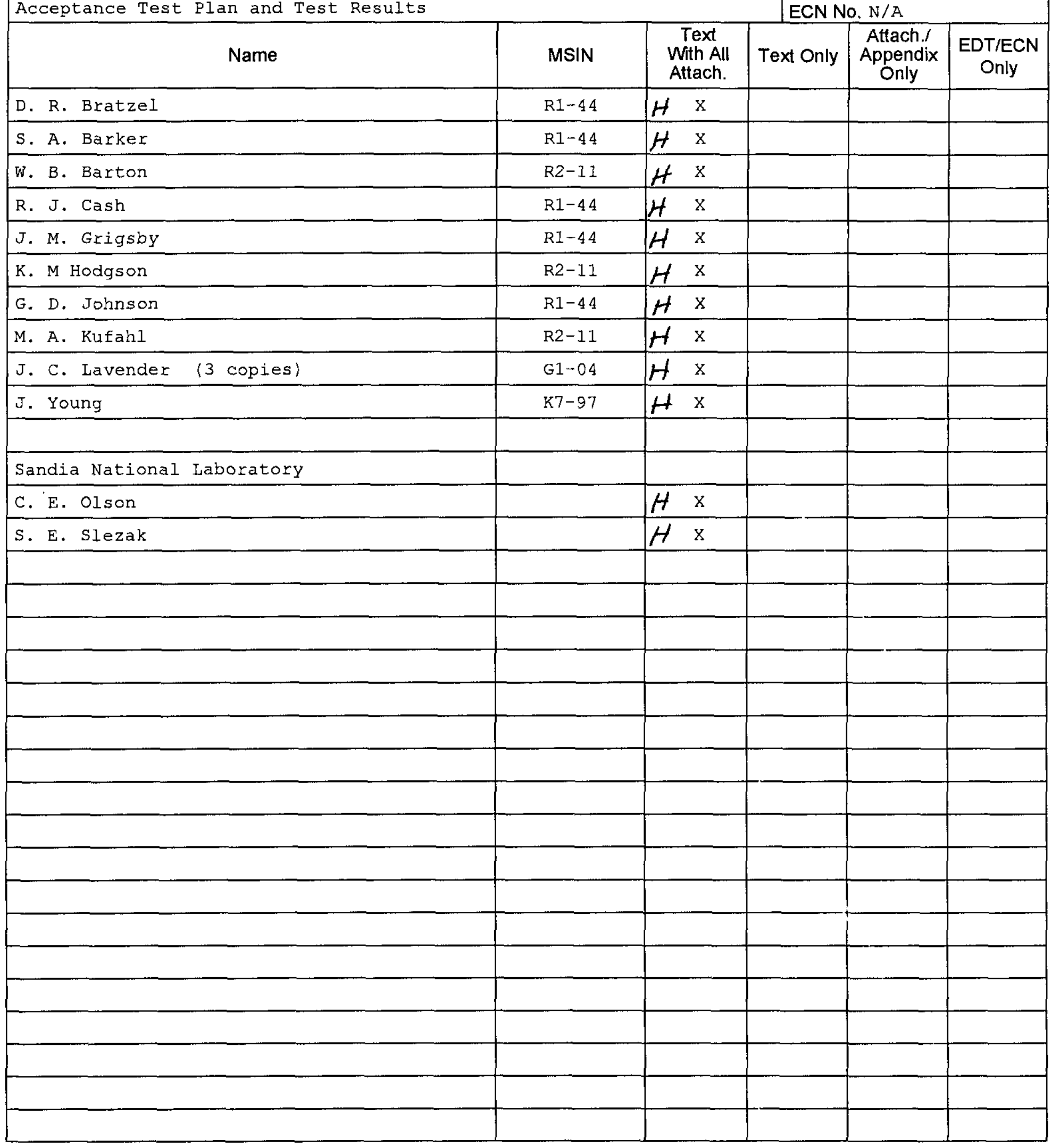

From

Nuclear Safety and Licensing

ECN No, N/A 


\section{RESOLVE! Version 2.5: Flammable Gas Accident Analysis Tool Acceptance Test Plan and Test Results}

J. C. Lavender

M. H. Chew \& Associates, Inc.

Richland, WA 99352

U.S. Department of Energy Contract DE-AC06 $99 R 214047$

EDT/ECN: 625133

Org Code: $74 \mathrm{~F} 00$

B\&R Code: EWP/2J2020
UC: $N / A$

Charge Code: 112892

Total Pages:
D. R. Bratzel

CH2M HILI Hanford Group, Inc.

Key Words: RESOLVE, flammable gas, analysis tool, computer model, gaseous release events, accident anlaysis, acceptnce testing

Abstract: RESOLVE! Version 2.5 is designed to quantify the risk and uncertainty of combustion accidents in DST's and SST's. The purpose of the acceptance testing is to ensure that all of the options and features of the computer code run; to verify that the calculated results are consistent with each other; and to evaluate the effects of the changes to the parameter values on the frequency and consequence trends associated with flammable gas deflagrations or detonations.

TRADEMARK DISCLAIMER. Reference herein to any specific commercial product, process, or service by trade name, trademark, manufacturer, or otherwise, does not necessarily constitute or imply its endorsement, recommendation, or favoring by the United States Government or any agency thereof or its contractors or subcontractors.

Printed in the United States of America. To obtain copies of this document, contact: Document Control Services, P.O. Box 950, Mailstop H6-08, Richland WA 99352, Phone (509) 372-2420; Fax (509) 376-4989.

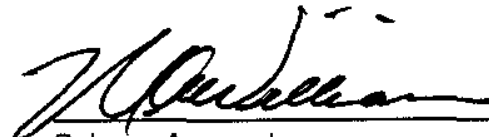

Release Approval

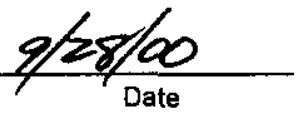

Date

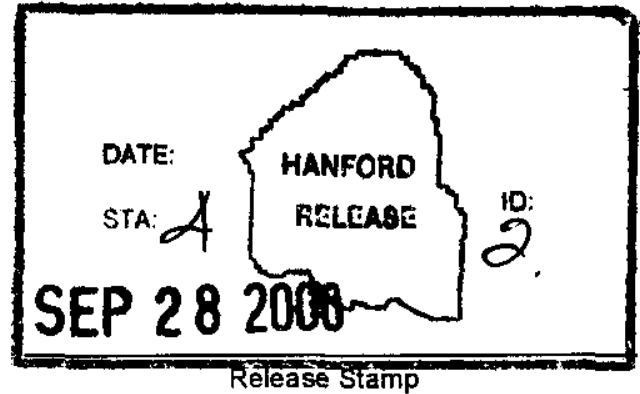

Rêlease stánp 
RPP-6888

Revision 0

\section{Resolve! Version 2.5:}

Flammable Gas Accident Analysis Tool Acceptance Test Plan and Test Results

Prepared for the U.S. Department of Energy

Assistant Secretary for Environmental Management

\section{CH2MHILL Hanford Group, Inc.}

Richland, Washington

Contractor for the U.S. Department of Energy

Office of River Protection under Contract DE-AC06-99RL14047 
LEGAL DISCLAIMER

This report was prepared as an account of work sponsored by an agency of the United States Government. Neither the United States Government nor any agency thereof, nor any of their employees, nor any of their contractors,

subcontractors or their employees, makes any warranty, express or implied, or assumes any legal liability or

responsibility for the accuracy, completeness, or any third party's use or the results of such use of any information, apparatus, product, or process disclosed, or represents that its use would not infringe privately owned rights. Reference herein to any specific commercial product, process, or service by trade name, trademark, manufacturer, or otherwise, does not necessarily constitute or imply its endorsement, recommendation, or favoring by the United States Government or any agency thereof or its contractors or subcontractors. The views and opinions of authors expressed herein do not necessarily state or reflect those of the United States Government or any agency thereof.

This report has been reproduced from the best available copy.

Available in paper copy and microfiche.

Available electronically at http://www doe.gov/bridge. Available for a processing fee to the U.S. Department of Energy and its contractors, in paper, from:

U.S. Department of Energy

Office of Scientific and Technical Information

P.O. Box 62

Oak Ridge, TN 37831-0062

phone: $865-576-8401$

fax: $865-576-5728$

email: reports@adonis.osti.gov(423) 576-8401

Available for sale to the public, in paper, from:

U.S. Department of Commerce

National Technical Information Service

5285 Port Royal Road

Springfield, VA 22161

Phone: 800-553-6847

fax: 703-605-6900

email: orders@ntis.fedworld.gov

online ordering:

http://www.ntis.gov/ordering.htm 


\title{
RESOLVE! VERSION 2.5: FLAMMABLE GAS ACCIDENT ANALYSIS TOOL ACCEPTANCE TEST PLAN AND TEST RESULTS
}

\author{
J. C. Lavender \\ M.H. Chew \& Associates, Inc. \\ Richland, Washington \\ M. A. Kufahl \\ Meier Enterprises, Inc. \\ Richland, Washington \\ S. A. Barker, D. R. Bratzel \\ CH2M HILL Hanford Group, Inc \\ Richland, Washington \\ J. Young \\ Pacific Northwest National Laboratory \\ Richland, Washington
}

Issued by

CH2M HILL Hanford Group, Inc.

Richland, Washington

For the

U.S. DEPARTMENT OF ENERGY

OFFICE OF RIVER PROTECTION

RICHLAND, WASHINGTON 


\section{RPP-6888 REV 0}

This page intentionally left blank. 


\section{RPP-6888 REV 0}

\section{EXECUTIVE SUMMARY}

To assist in quantifying the potential risk of flammable gas accidents in the Hanford waste tanks an Analysis Tool (AT) has been developed using commercially available software and tank characterization data. The AT is a computer code called RESOLVE!. The AT is designed to quantify the risk and uncertainty of combustion accidents in actual or representative tanks and the change in risk that would result from using different control strategies. RESOLVE! is used to help identify optimal controls for each tank and analyze the implementation of the controls prior to actual implementation in the field.

The acceptance and validation testing of a developmental version, RESOLVE! Version 2.13 Acceptance Testing: Test Plan and Test Results is documented in Attachment 1. Following independent reviews of the AT Version 2.13 additional changes were identified and have been incorporated. The new revision, RESOLVE! Version 2.5 has been issued. The changes incorporated include revised waste classifications based on the characteristics of the waste, incorporation of empirical or observed tank waste behavior data, and graphical user interface upgrades.

Each Acceptance Test Plan is divided into three primary areas. The three areas are: "Features Testing," "Characteristics Testing," and "Trend Testing." The purpose of the "Features Testing" is to ensure that all of the options and features of the computer code run. The purpose of the "Characteristics Testing" is to verify that the calculated results are consistent with each other and that the results are repeatable and to quantify the baseline stability or behavior of the computer code. "Trend Testing" is performed to evaluate the effects of the changes to the parameter values on the frequency and consequence trends (i.e. increase, decrease or no change) associated with flammable gas deflagrations or detonations.

The results of the Acceptance Testing indicate that the majority of the parameters modified for both double-shell tanks (DSTs) and single-shell tanks (SSTs) did not significantly affect the benchmark results. This is the same conclusion reached during acceptance testing of Resolve Version 2.13. The parameter modifications that did significantly impact the results 


\section{RPP-6888 REV 0}

include waste volume reduction, an increase or decrease in ventilation flow rate and changes in the waste and waste gas generation characteristics ( for DSTs only). In each of these cases the results trend as expected.

As a result of testing there were two limitations identified. One resulted from "Characteristics Testing" and determined that the calculated mean toxicological sum of fractions exceeded the acceptance relative standard deviation criteria. It is recommended that prior to reporting toxicological consequences, multiple runs should be performed using different statistical sampling parameters and the results evaluated for appropriateness. The other limitation associated with "Waste Intrusive Equipment" is based on a lack of actual or observed data to validate the results. 


\section{TABLE OF CONTENTS}

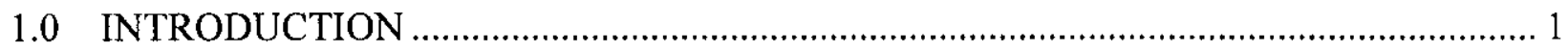

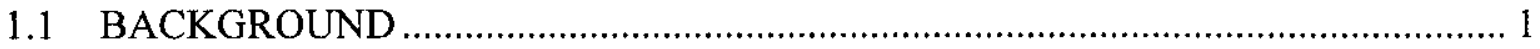

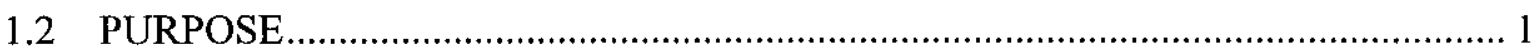

2.0 ACCEPTANCE TEST PLAN

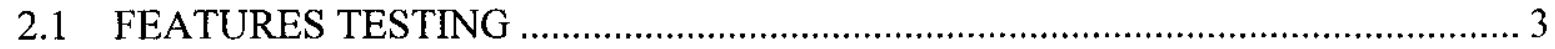

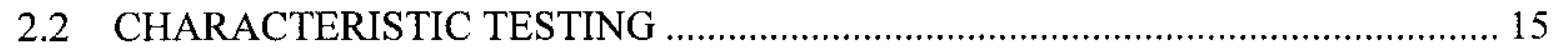

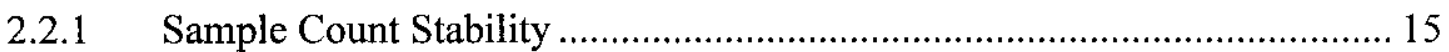

2.2.2 Sample Seed Stability ………………………..................................... 16

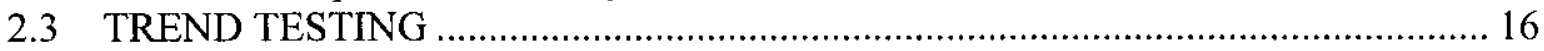

2.3.1 Saltwell Pumping ............................................................................. 29

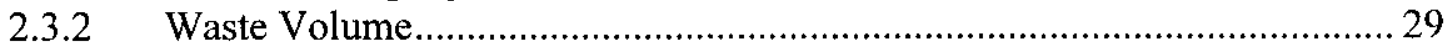

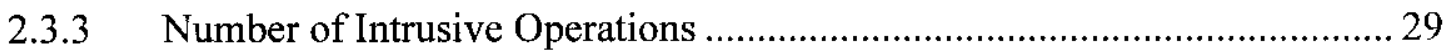

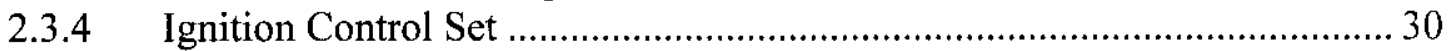

2.3.5 Ventilation Rate ……………………………...................................... 30

2.3.6 Inerting the Tank Headspace with Nitrogen ................................................. 30

2.3.7 Tank Failure (Cracking) Pressure …………................................................ 31

2.3.8 Tank Failure (Collapse) Pressure ............................................................. 31

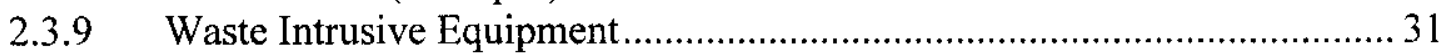

2.3.10 Increase in Bulk Density Ratio of Solids to Liquids .................................. 32

2.3.11 Increase in Waste Gas Generation Rate .................................................... 32

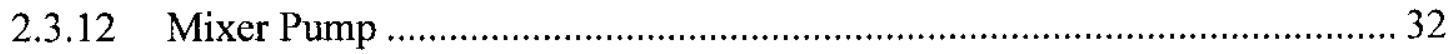

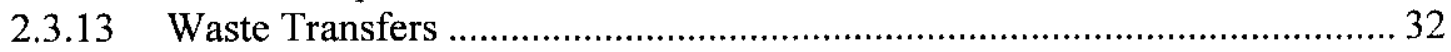

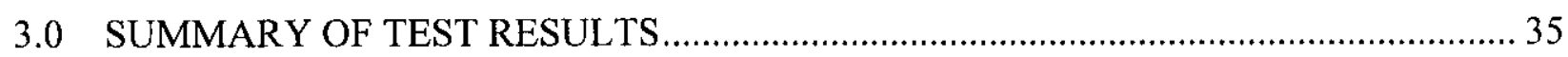

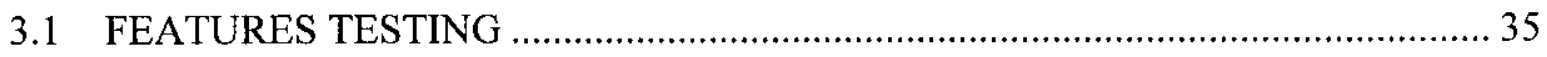

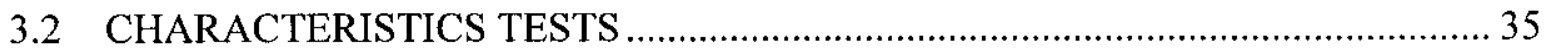

3.3 SUMMARY OF TREND TESTING RESULTS …………………..........................35

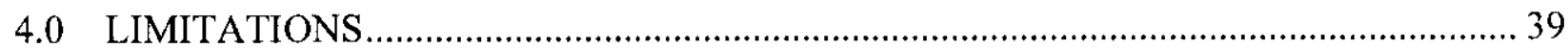

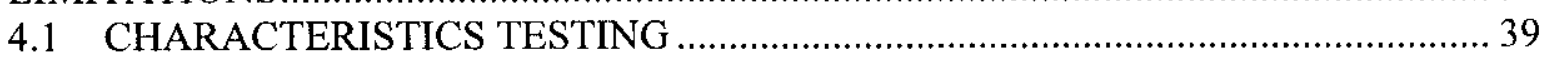

4.2 WASTE INTRUSIVE EQUIPMENT ……………….......................................... 39

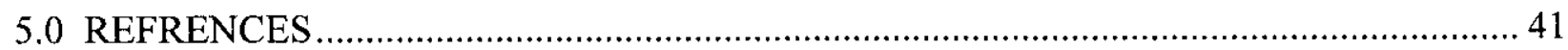

\section{APPENDICES}

A SST ACCEPTANCE TEST RESULTS ..................................................................... A-

B DST ACCEPTANCE TEST RESULTS ....................................................................... 


\section{RPP-6888 REV 0}

\section{LIST OF TABLES}

Table 1. Single-Shell Tanks: Acceptance Tests and Input Variables and Parameters ............................................................................. 11

Table 2. Double-Shell Tanks: Acceptance Tests and Input Variables and Parameters ...............................................................................15

Table 3. Summary of Single Shell Tanks Used in Trend Testing...............................................23

Table 4. Summary of Double Shell Tanks Used in Trend Testing .............................................24

Table 5. Single Shell Tanks: Expected Results from Acceptance …….................................... 25

Table 6. Double Shell Tanks: Expected Results from Acceptance Testing.................................27

Table 7. Waste Transfer Input Parameters............................................................................

Table 8. Summary Results of the Characteristics Tests Performed ..............................................37 


\section{RPP-6888 REV 0}

\section{LIST OF FIGURES}

Figure1. Comparison of A-102 Benchmark Events, Deflagrations and Detonations to Modified Conditions

Figure 2. Comparison of A-102 Benchmark GRE Frequency to Modified Conditions

Figure 3. Comparison of B-111 Benchmark Events, Deflagrations and Detonations to Modified Conditions

Figure 4. Comparison of B-111 Benchmark GRE Frequency to Modified Conditions $\mathrm{F}-4$

Figure 5. Comparison of S-102 Benchmark Events, Deflagrations and Detonations to Modified Conditions

Figure 6. Comparison of S-102 Benchmark GRE Frequency to Modified Conditions F-6

Figure 7. Comparison of T-203 Benchmark Events, Deflagrations and Detonations to Modified Conditions

Figure 8. Comparison of T-203 Benchmark GRE Frequency to Modified Conditions F-8

Figure 9. Comparison of A-102 Benchmark Accident Mean Frequency to Modified Conditions F-9

Figure 10. Comparison of A-102 Benchmark Accident Onsite Consequences to Modified Conditions F-10

Figure 11. Comparison of A-102 Benchmark Accident Expected Risk to Modified Conditions F-11

Figure 12. Comparison of B-111 Benchmark Accident Mean Frequency to Modified Conditions

Figure 13. Comparison of B-111 Benchmark Accident Onsite Consequences to Modified Conditions F-13

Figure 14. Comparison of B-111 Benchmark Accident Expected Risk to Modified Conditions 
RPP-6888 REV 0

\section{LIST OF FIGURES}

Figure 15. Comparison of S-102 Benchmark Accident Mean Frequency to Modified Conditions

Figure 16. Comparison of S-102 Benchmark Accident Onsite Consequences to Modified Conditions

Figure 17. Comparison of S-102 Benchmark Accident Expected Risk to Modified Conditions.

Figure 18. Comparison of AN-107 Benchmark Events, Deflagrations and Detonations to Modified Conditions

Figure 19. Comparison of AN-107 Benchmark GRE Frequency to Modified Conditions

Figure 20. Comparison of AW-106 Benchmark Events, Deflagrations and Detonations to Modified Conditions.

Figure 21. Comparison of AW-106 Benchmark GRE Frequency to Modified Conditions

Figure 22. Comparison of SY-101 Benchmark Events, Deflagrations and Detonations to Modified Conditions.

Figure 23. Comparison of SY-101 Benchmark GRE Frequency to Modified Conditions

Figure 24. Comparison of AN-107 Benchmark Accident Mean Frequency to Modified Conditions

Figure 25. Comparison of AN-107 Benchmark Accident Onsite Consequences to Modified Conditions F-25

Figure 26. Comparison of AN-107 Benchmark Accident Expected Risk to Modified Conditions F-26

Figure 27. Comparison of AW-106 Benchmark Accident Mean Frequency to Modified Conditions F-27

Figure 28. Comparison of AW-106 Benchmark Accident Onsite Consequences to Modified Conditions F-28 


\section{RPP-6888 REV 0}

\section{LIST OF FIGURES}

Figure 29. Comparison of AW-106 Benchmark Accident Expected Risk to Modified Conditions

Figure 30. Comparison of SY-101 Benchmark Accident Mean Frequency to Modified Conditions

Figure 31. Comparison of SY-101 Benchmark Accident Onsite Consequences to Modified Conditions F-31

Figure 32. Comparison of SY-101 Benchmark Accident Expected Risk to Modified Conditions 


\section{RPP-6888 REV 0}

This page intentionally left blank. 


\subsection{INTRODUCTION}

\subsection{BACKGROUND}

To assist in quantifying the potential risk of flammable gas accidents in the Hanford waste tanks, an Analysis Tool (AT) has been developed using commercially available software and tank characterization data. In addition to using available data and analysis results, the process also uses formal systematic expert elicitation on flammable gas technical parameters for which no data exists. The AT is a computer code called RESOLVE!. The AT is designed to quantify the risk and uncertainty of combustion accidents in actual or representative tanks and the change in risk that would result from using different control strategies. RESOLVE! is used to help identify optimal controls for each tank and analyze the implementation of the controls prior to actual implementation in the field.

The acceptance and validation testing of the developmental versions, RESOLVE! Version 1.51, is documented in RESOLVE! Version 1.51 Acceptance Testing: Test Plan and Test Results (Lavender et al. 1998) and RESOLVE! Version 2.13 Acceptance Testing: Test Plan and Test Results (see Attachment 1). RESOLVE! Version 1.51 was modified primarily to include the analysis and evaluation of Double-Shell Tanks (DSTs). Additional changes to the refined safety AT (Version 1.51) included a buoyant displacement model, revised mass balance, waste intrusive equipment burns, mixer pump, waste transfer and the graphical user interface (GUI). The modified version was identified as RESOLVE! Version 2.13. The impacts associated with these changes have been previously determined and are documented (Slezak et al. 1999, Slezak and Bratzel 1997). The acceptability of RESOLVE! Version 2.13 is documented in Attachment 1.

Following independent reviews of the AT Version 2.13 additional changes were identified and have been incorporated. The new revision, RESOLVE! Version 2.5 has been issued. The changes incorporated include revised waste classifications based on the characteristics of the waste, incorporation of empirical or observed tank waste behavior data, and the graphical user interface (GUI) upgrades. The impacts associated with these changes have been previously determined and are documented (Slezak et al. 2000). This report documents the acceptance testing performed on Version 2.5.

\subsection{PURPOSE}

The acceptance test is part of the software certification process for RESOLVE! Version 2.5. This testing has been performed to enhance the understanding of the analysis framework (AF) and implication of the AT results. The results of acceptance testing are used to do the following:

Confirm that the results of RESOLVE! Version 2.5 trend logically

- $\quad$ Ensure that RESOLVE! Version 2.5 performs within the predefined parameters 
- Determine the status of previously identified AT user interface issues and calculational errors

- Identify any limitations for application of Version 2.5.

Whenever an area of acceptance testing raises an issue, two approaches are used to evaluate the condition. Unless it is evident that an error (e.g., computational or GUI) exists, the code developers and the Tank Farm Contractor team convene to determine if the suspected error is correct (i.e., whether the tool behaved as expected based on the parameter modified and the correct calculational formulae) or whether a deficiency exists (i.e., results are unexpected based on parameter modification and the calculational formulae). If a deficiency has been noted, a code limitation (see Section 4.0) is described. Section 2.0 identifies the tests performed, Section 3.0 summarizes testing results, Section 5.0 lists references and Appendix A and B provide detailed trend test analysis results. 


\subsection{ACCEPTANCE TEST PLAN}

The acceptance testing performed using Version 2.13 is documented in Attachment 1. This was an extensive test of all features of the AT and GUI and trended the calculated results for each change in control strategy, operation, and waste modification using multiple single-shell tanks (SSTs) and double-shell tanks (DSTs). The acceptance test plan for Version 2.5 is designed to evaluate the same changes in control strategies, operations, and waste modifications to corroborate the results reached during acceptance testing of Version 2.13. That is, the results are not trended at the conclusion of Version 2.5 acceptance testing.

Two acceptance test plans (ATPs) have been developed, one addresses the SSTs and the other addresses the DSTs. To provide a better understanding of the behavior of the AT the two test plans evaluate the same parameter changes. Where appropriate additional tests have been identified based on the characteristics of the waste contained in the tanks. For example, buoyant displacement gaseous release events are only evaluated with DSTs. Tables 1 and 2 identify the tests and input parameters, and variables modified for each test that will be performed, for SSTs and DSTs, respectively.

Each ATP is divided into three primary areas. The three areas are: "Features Testing," "Characteristics Testing," and "Trend Testing." The purpose of the "Features Testing" (see Section 2.1) is to ensure that all of the options and features of the computer code run. The purpose of the "Characteristics Testing" (see Section 2.2) is to verify that the calculated results are consistent with each other and repeatable and to quantify the baseline stability or behavior of the computer code. "Trend Testing" (see Section 2.3) is performed to evaluate the effects of the changes to the parameter values on the frequency and consequence trends (i.e. increase, decrease or no change) associated with gas deflagrations or detonations.

Code limitations and issues identified during testing are discussed and summarized in Section 4.0.

\subsection{FEATURES TESTING}

Testing the features of RESOLVE! 2.5 ensures that it runs correctly and produces the proper outputs when various available options are selected. An extensive test of the AT and GUI features is discussed in Attachment 1. The "Features Testing" performed in this ATP is limited to the options selected during characteristics and trend testing. This limited set of features tests based on the parameter modifications, will also verify that the code and data libraries are consistent with the changes in the baseline documentation.

As shown in Table 2 and Attachment 1, additional features tests are identified for DSTs. The additional DST features test include turning on the mixer pump (normally off) and creating buoyant displacement gaseous release events (GREs) in non-buoyant tanks by modifying the characteristics of the waste. 


\section{RPP-6888 REV 0}

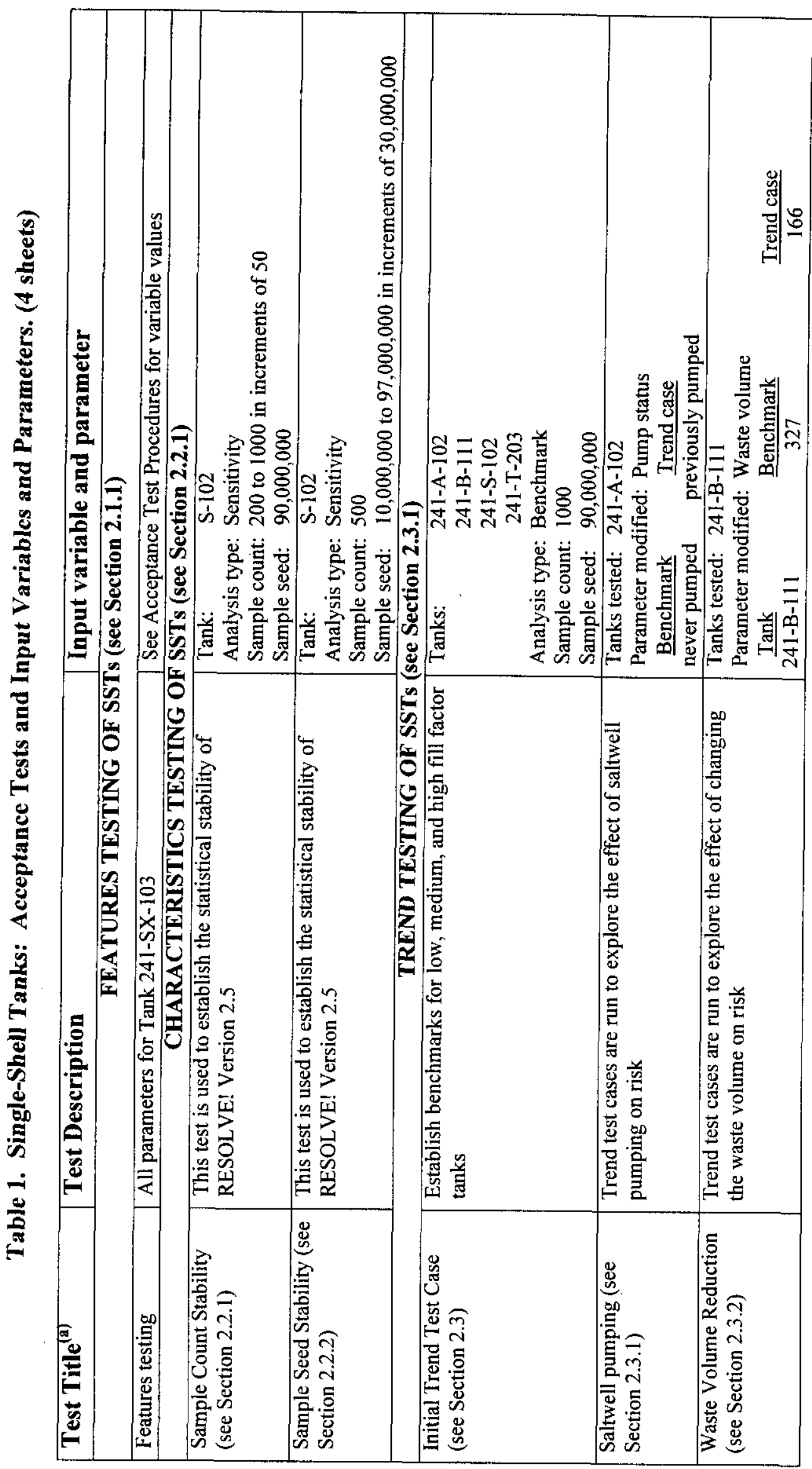




\section{RPP-6888 REV 0}

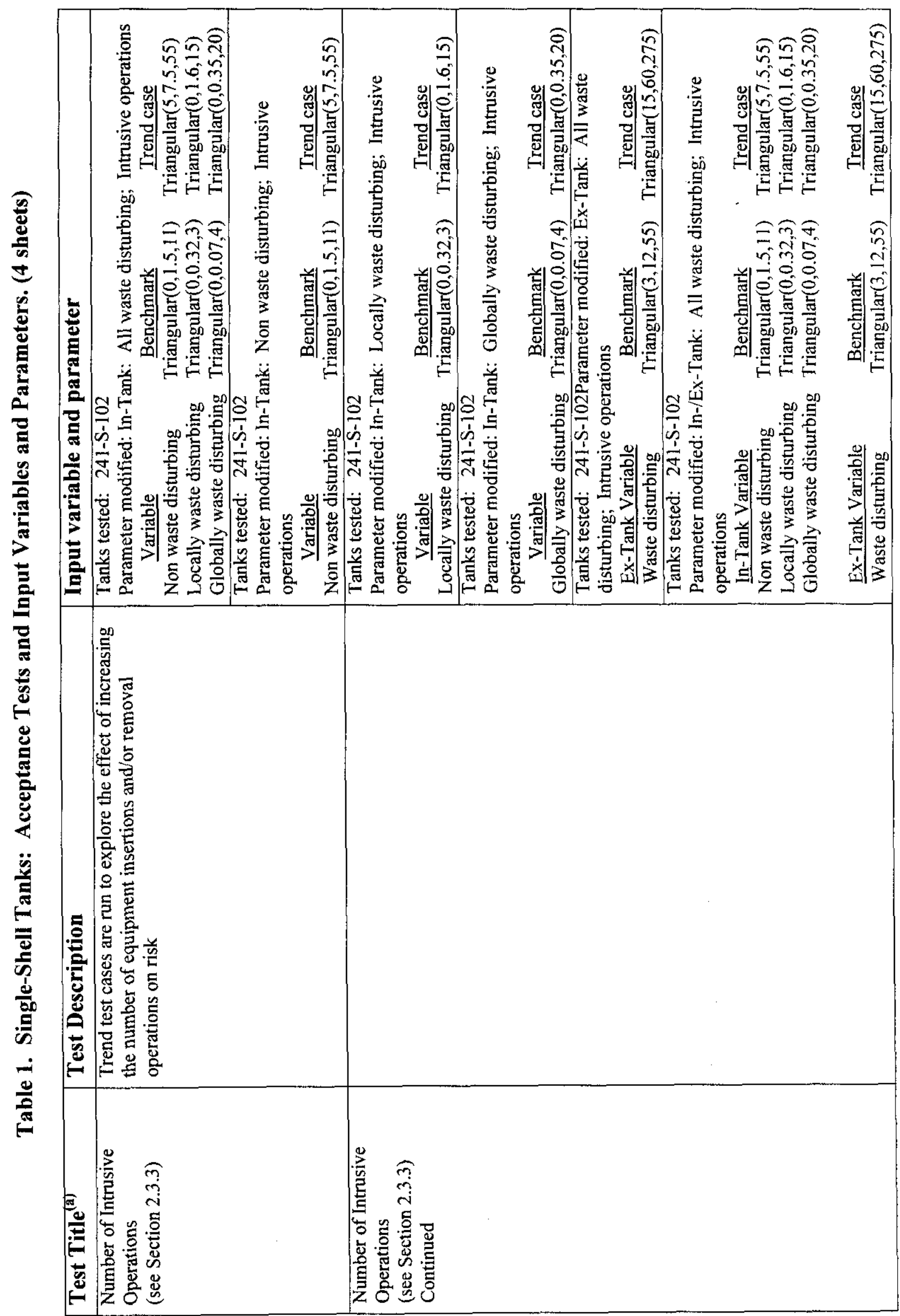


RPP-6888 REV 0

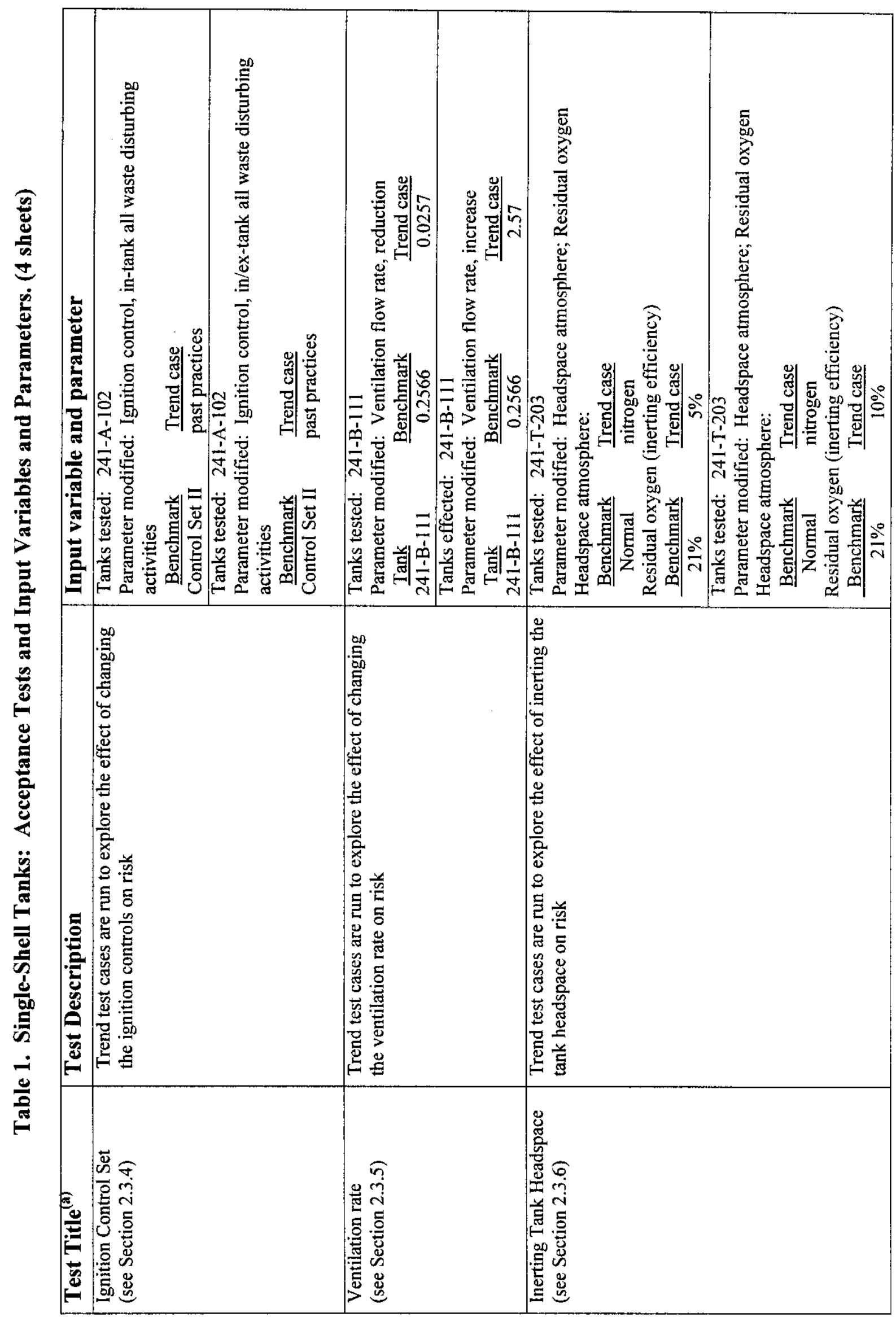


RPP-6888 REV 0

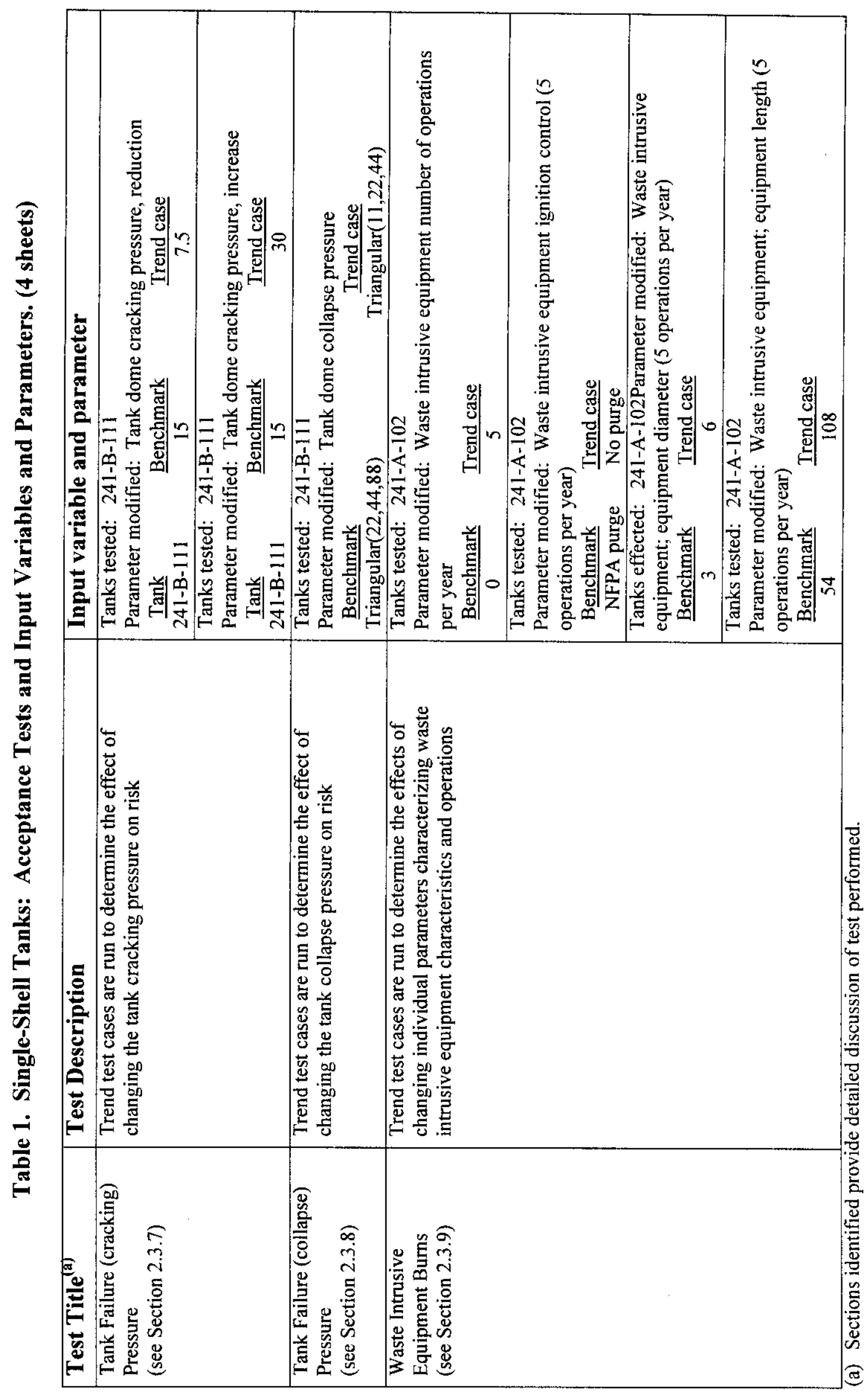




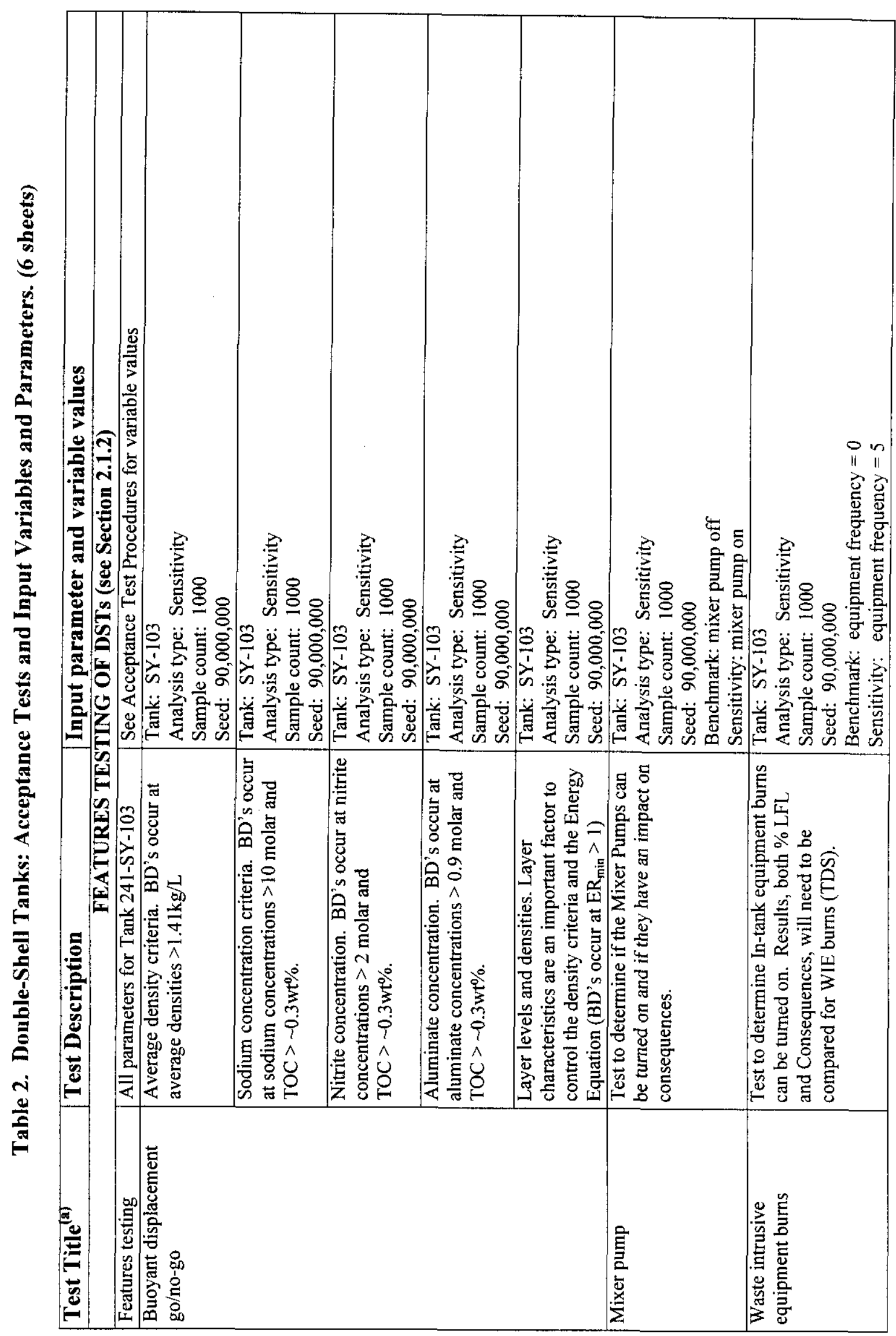


RPP-6888 REV 0

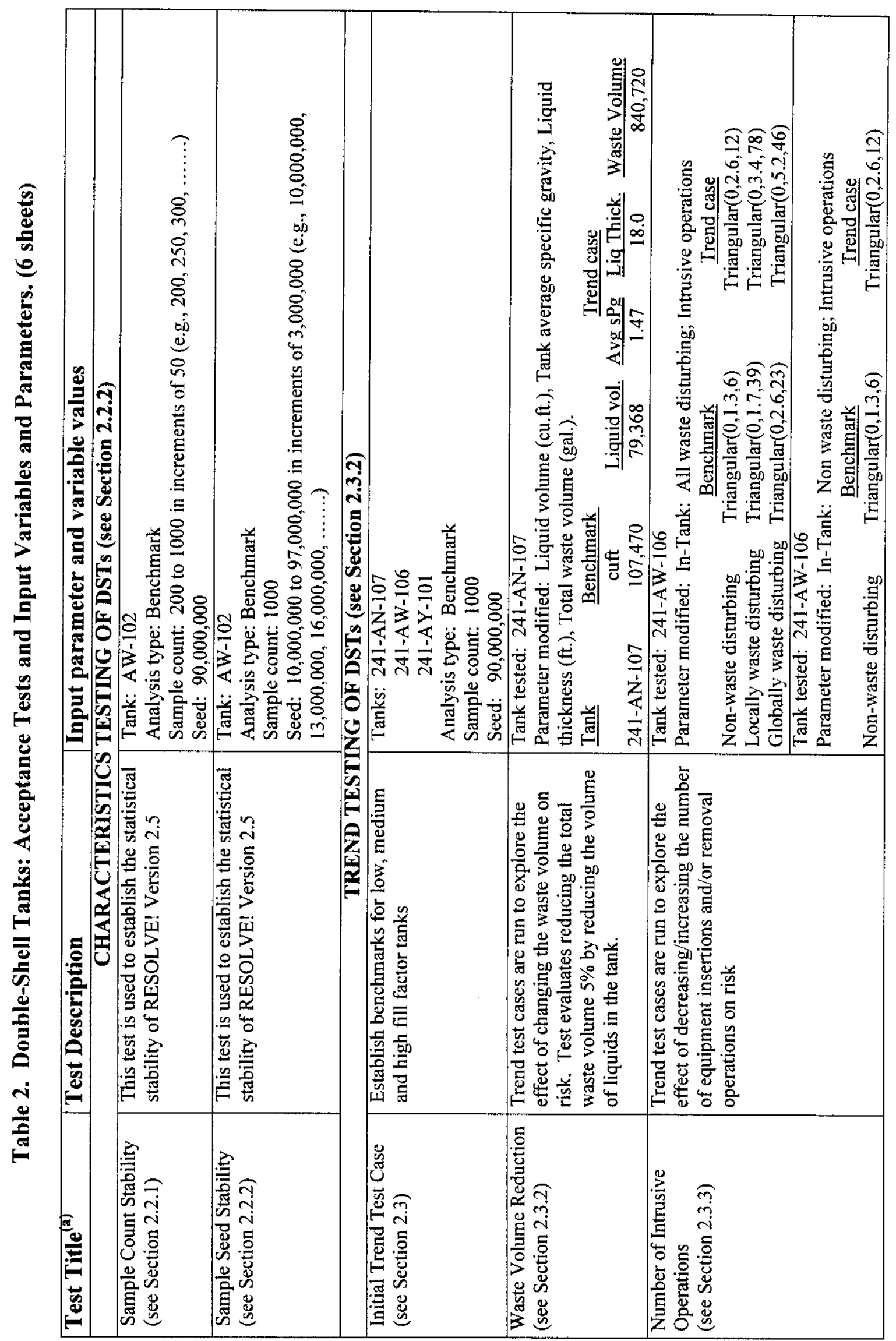


RPP-6888 REV 0

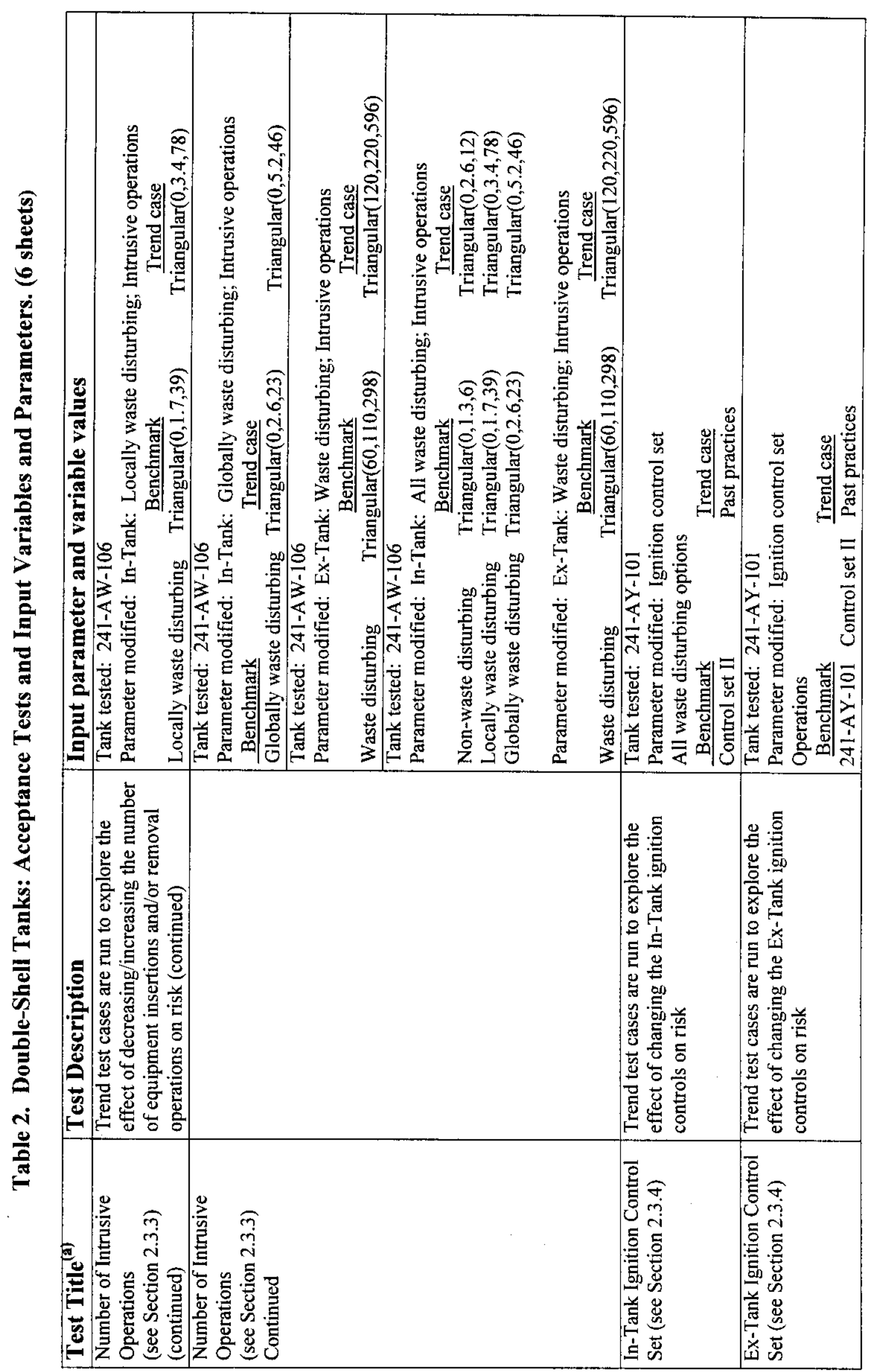


RPP-6888 REV 0

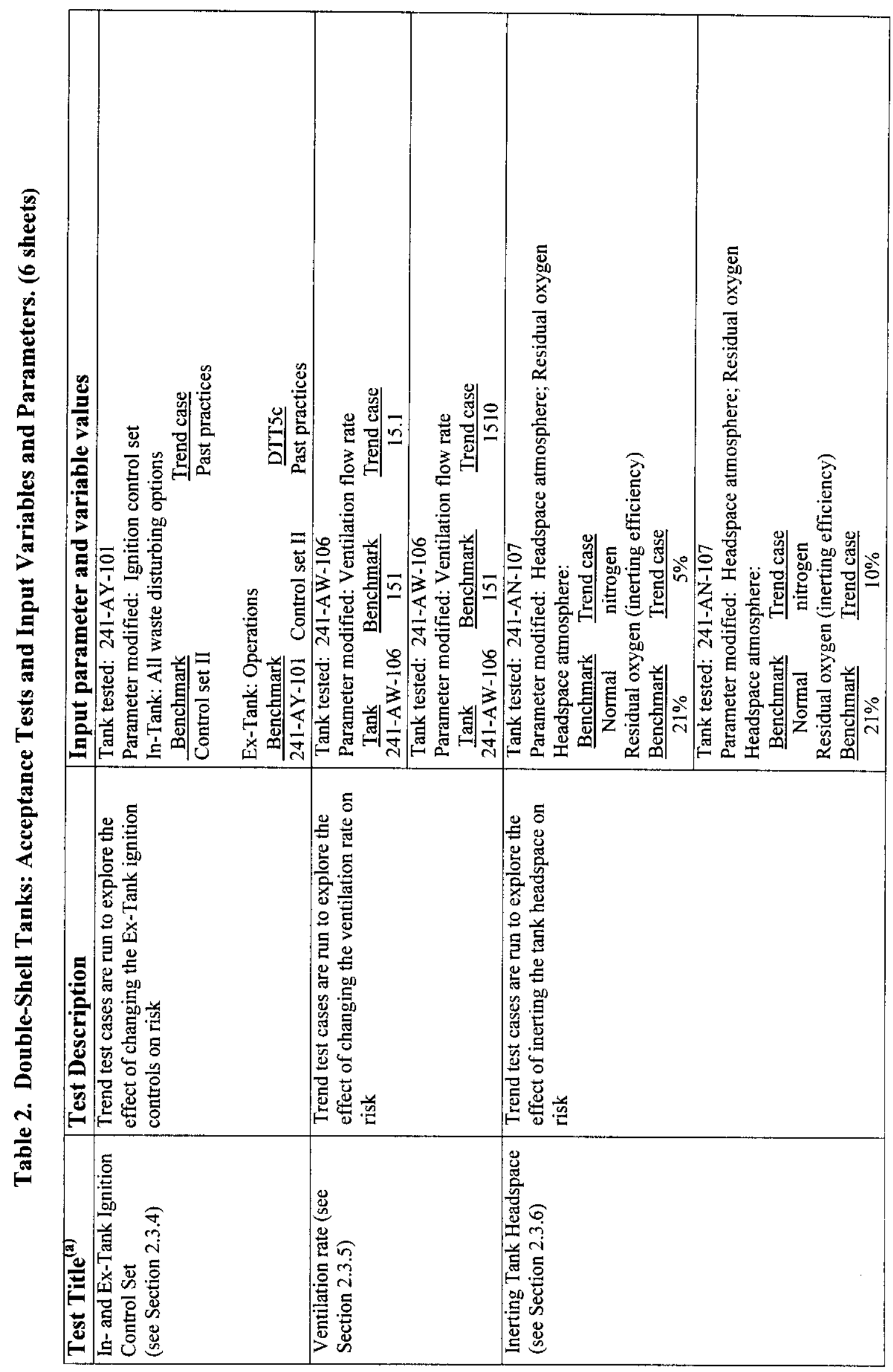


RPP-6888 REV 0

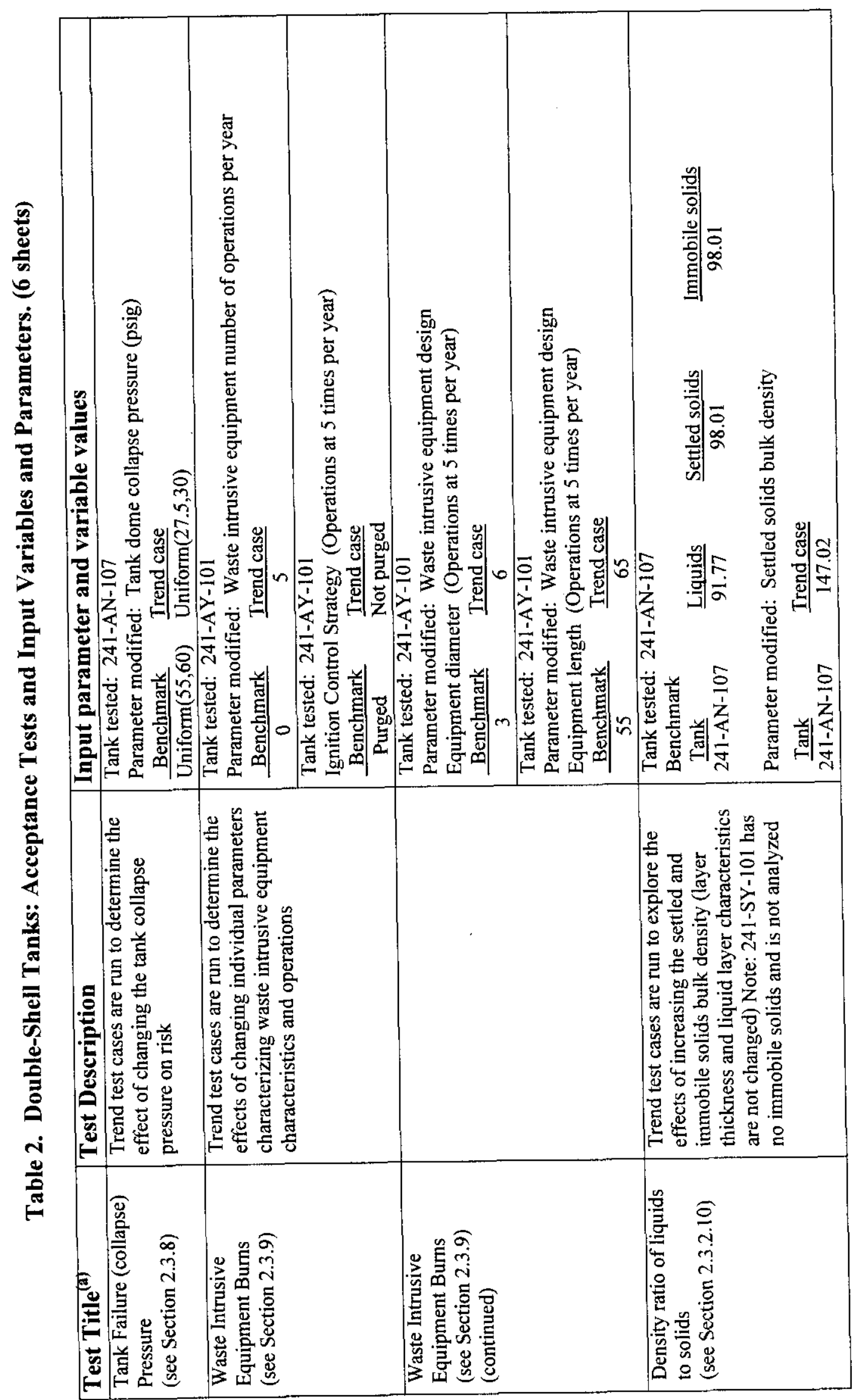


RPP-6888 REV 0

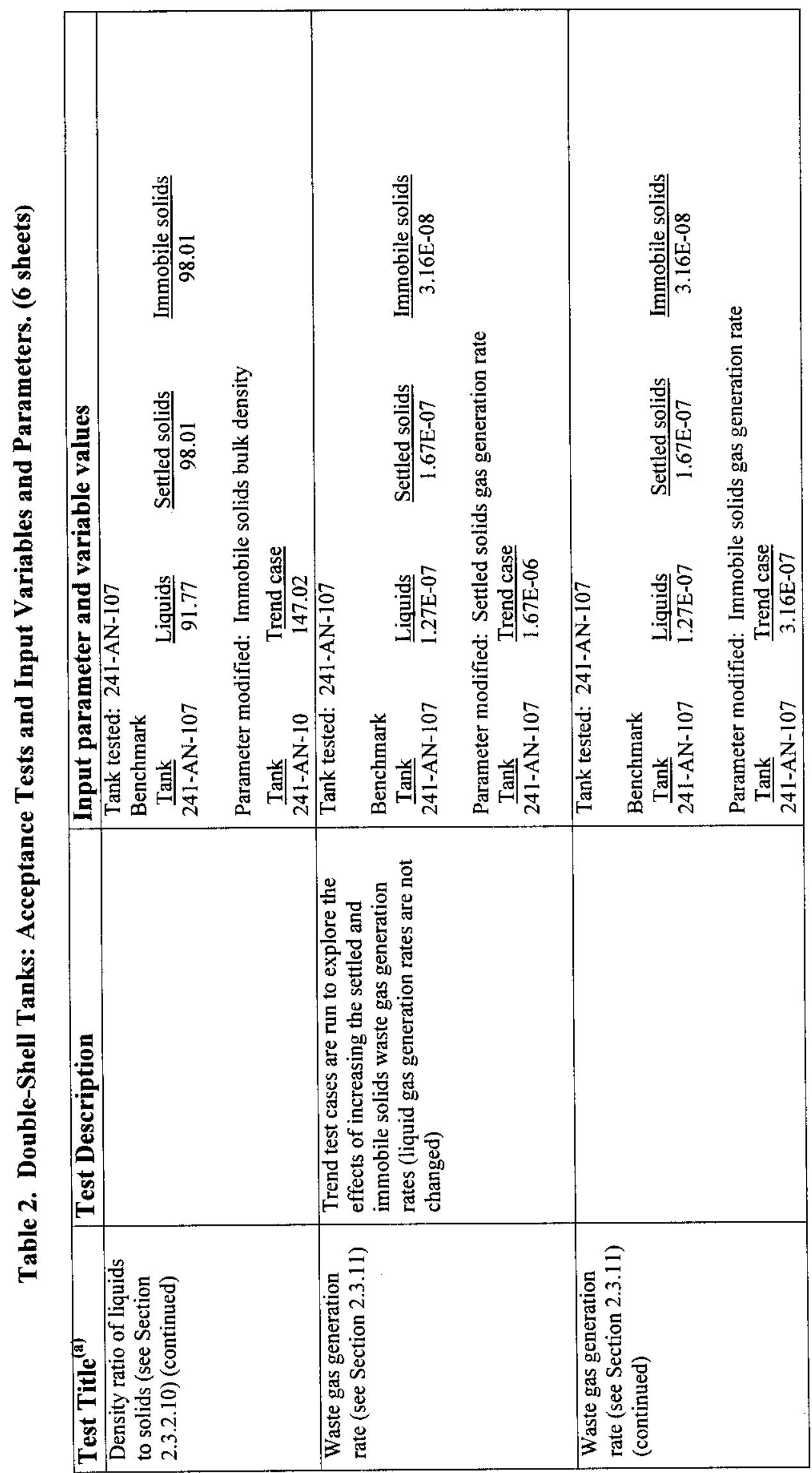


RPP-6888 REV 0

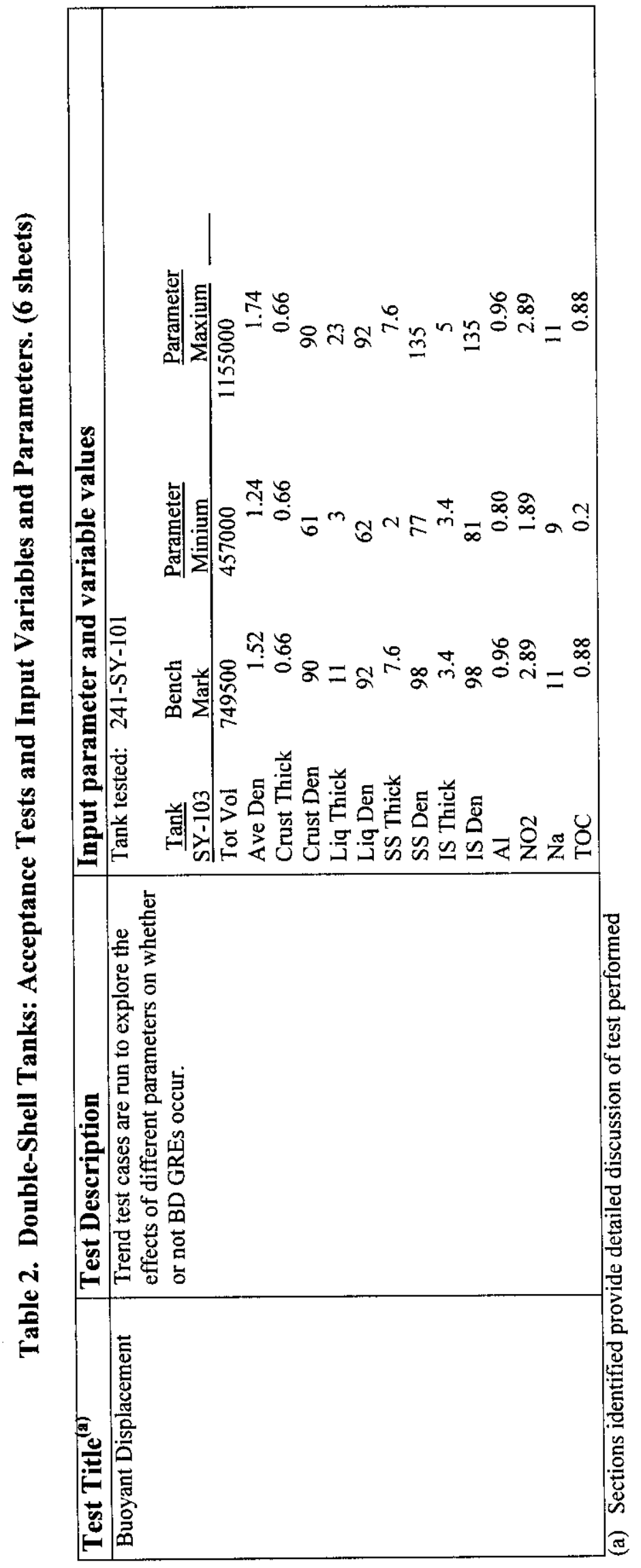


Acceptable behavior is that all features perform the function intended by the programmers and that no feature causes the program to "crash" or result in an error message. The baseline code and the data libraries must also be consistent with the documentation. Results of "Feature Testing" are summarized in Section 3.1. Any code limitations generated as a result of this testing are presented in Section 4.0.

\subsection{CHARACTERISTIC TESTING}

Characteristic Testing is performed following the successful completion of the "Features Testing." The purpose of the "Characteristic Testing" is to verify that the calculated results are stable and repeatable and to determine the number of trials (or samples) required to achieve stable and repeatable results. These tests are performed at different workstations using the identical statistical analysis parameters. Characteristics Testing is also used to establish system baseline stability or behavior due to changes in statistical analysis parameters. Typically, it would be necessary to test the AT as an integrated tool; however, DSTs, are analyzed using a different set of algorithms. Therefore, it is necessary to test both the SST and DST stability. The following subsections describe the characteristics testing, which is common to both SSTs and DSTs and the expected changes to the results caused by the testing. Results of Characteristic Testing are summarized in Section 3.2.

To test system stability, the variance, standard deviation and the relative standard deviation values are calculated. The relative standard deviation is calculated using two methods:

1) dividing the square root of the variance by the average and 2) dividing the standard deviation by the average. Simply stated, the standard deviation equals the square root of the variance. In order to comply, the values must not vary by more than a factor or two. Translated to the percentile notation common with relative standard deviations, the values cannot be over $200 \%$.

\subsubsection{Sample Count Stability}

This test is used to establish the stability of RESOLVE! 2.5 . That is, changing the number of samples analyzed will impact the statistical analysis and the reported results. It is expected that the reported results for each case based on the number of samples analyzed will vary; however, the variance should not be significant. An analyst performs benchmark runs using a single tank and the same sample seed value. For each run, the sample count is iterated from 200 to 1000 in increments of 50 .

The mean of the onsite radiological and toxicological results, as shown on the GUI, are compared, for the benchmark runs. The relative standard deviation should be less than $200 \%$. See Tables 1 and 2 for a description of the tests performed. 


\section{RPP-6888 REV 0}

\subsubsection{Sample Seed Stability}

Similar to the Sample Count Sensitivity test discussed above, changing the seed value will impact the statistical analysis and the reported results. It is expected that the reported results for each sample seed value analyzed will vary; however, the variance should not be significant. An analyst performs benchmark runs using a single tank and the same sample count. For each run, the sample seed is increased from 10,000,000 to $61,000,000$ in increments of 300,000 .

The mean of the onsite radiological and toxicological results as shown on the GUI for all runs are compared. The relative standard deviation should be less than $200 \%$. See Tables 1 and 2 for a description of the tests performed.

\subsection{TREND TESTING}

Trend testing is performed to evaluate the effects of changes in the pedigreed data values and the predefined controls on the frequency and consequences of a flammable gaseous release. The trend testing is performed by comparing the Benchmark case to the modified (i.e., changed, pedigreed data values and predefined controls) case. As discussed previously in Section 1.1, the major improvement of the refined safety AT is revision of the tank waste characteristics. Four representative SSTs and three DTSs were selected for trend analysis. Tables 3 and 4 for SSTs and DSTs, respectively, identify the tanks and the parameters selected for trend testing.

For this test a series of tanks was selected based on the classification of the waste. That is, prior versions of RESOLVE! have classified tanks using Facility Groups (HNF 1999). The waste characteristics; i.e., composition and GRE behavior, have been revised in the relational database (Barker et al. 2000) to reflect actual waste conditions. The four SSTs and three DSTs selected for testing have different waste characteristics, thus the results due to changes in the waste parameters, tank operations, and controls are reviewed to determine if the results are as expected.

The general approach followed in trend testing is to develop a Benchmark case for each tank maximizing the sensitivity of the program and to perform multiple analyses or sensitivity case runs for each tank changing only one parameter (i.e., relational database data value or predefined control) per analysis. The output from each run or analysis (e.g., frequency and/or consequences) are tabulated and compared. This approach will test the effects of significantly increasing or decreasing a parameter.

Tables 1 and 2 for SSTs and DSTs, respectively, provide a listing of the input parameters and values that are changed for each test. Each trend test uses the Benchmark Case as a template. That is, the Benchmark Case file is "opened" and modified. For all trend tests the Analysis Type is changed from "Benchmark" to "Sensitivity" and the appropriate input parameters and values are changed to the values specified for each test.

The following subsections describe the trend testing. The expected results due to the parameter changes are shown in Tables 5 and 6 for SSTs and DSTs, respectively. Based on the expected, 
Table 3. Summary of Single Shell Tanks Used in Trend Testing.

\begin{tabular}{|c|c|c|c|c|c|c|c|}
\hline Tank & $\begin{array}{c}\text { Tank } \\
\text { Volume } \\
(\mathbf{a})\end{array}$ & $\begin{array}{c}\text { Waste } \\
\text { Volume } \\
(\mathbf{a})\end{array}$ & $\begin{array}{c}\text { Waste } \\
\text { Classification } \\
\text { (b) }\end{array}$ & $\begin{array}{c}\text { Fill } \\
\text { Factor }\end{array}$ & $\begin{array}{c}\text { Saltwell } \\
\text { Pump } \\
\text { Status }\end{array}$ & $\begin{array}{c}\text { Ventilation } \\
\text { Flow Rate } \\
\text { (cfm) }\end{array}$ & $\begin{array}{c}\text { GRE } \\
\text { Behavior } \\
\text { (c) }\end{array}$ \\
\hline B-111 & 530,000 & 237,000 & SL-NL & $\begin{array}{c}0.3 \\
\text { (medium) }\end{array}$ & $\begin{array}{c}\text { never } \\
\text { pumped }\end{array}$ & 0.3 & NBD \\
\hline S-102 & 758,000 & 549,000 & SC/SS-NL & $\begin{array}{c}0.5 \\
\text { (medium) }\end{array}$ & $\begin{array}{c}\text { never } \\
\text { pumped }\end{array}$ & 0.2 & NBD \\
\hline U-111 & 530,000 & 329,000 & MIX-NL & $\begin{array}{c}0.4 \\
\text { (medium) }\end{array}$ & $\begin{array}{c}\text { never } \\
\text { pumped }\end{array}$ & 0.2 & NBD \\
\hline T-203 & 38,000 & 35,000 & SL-NL & $\begin{array}{c}0.9 \\
\text { (high) }\end{array}$ & $\begin{array}{c}\text { never } \\
\text { pumped }\end{array}$ & 0.4 & NBD \\
\hline
\end{tabular}
(a) Volumes shown are approximate.
(b) Taken from RESOLVE! Version 2.5 database (Barton et al. 1998).
(c) SL - slurry
$\mathrm{NL}$ - no liquid
$\mathrm{SC}$ - salt cake
SS - salt sludge
(d) GRE - Gas release event
NBD - Non-buoyant displacement 
RPP-6888 REV 0

Table 4. Summary of Double Shell Tanks Used in Trend Testing.

\begin{tabular}{|c|c|c|c|c|c|c|c|}
\hline \multirow[t]{2}{*}{ Tank } & \multirow{2}{*}{$\begin{array}{c}\text { Waste } \\
\text { Volume }^{(a)}\end{array}$} & \multirow{2}{*}{$\begin{array}{c}\text { Waste } \\
\text { Classification }^{(b)}\end{array}$} & \multicolumn{3}{|c|}{ Waste Characteristics $^{(c)}$} & \multirow{2}{*}{$\begin{array}{c}\text { Ventilation } \\
\text { Flow Rate } \\
\text { (cfm) }\end{array}$} & \multirow{2}{*}{$\begin{array}{c}\text { GRE } \\
\text { Behavior }^{(c)}\end{array}$} \\
\hline & & & $\begin{array}{c}\text { Volume } \\
\left(\mathrm{ft}^{3}\right)\end{array}$ & $\begin{array}{c}\text { Gas } \\
\text { Generation } \\
\text { Rate } \\
\text { (moles } / \mathrm{m}^{3} \\
\text { sec) }\end{array}$ & $\begin{array}{c}\text { Bulk } \\
\text { Density } \\
\left(\mathbf{l b m} / \mathrm{ft}^{3}\right)\end{array}$ & & \\
\hline \multirow[t]{4}{*}{$\mathrm{AW}-106$} & \multirow[t]{4}{*}{579,300} & \multirow[t]{4}{*}{ SC/SS-NL } & $\mathrm{C}-0$ & C-NA & C-NA & \multirow[t]{4}{*}{151} & \multirow[t]{4}{*}{ NBD } \\
\hline & & & $\mathrm{L}-46,960$ & $\overline{\mathrm{L}}-3.17 \mathrm{E}-09$ & L-69.3 & & \\
\hline & & & SS-30,480 & SS-1.13E-8 & SS-98.01 & & \\
\hline & & & IS-0 & IS-NA & IS-NA & & \\
\hline \multirow[t]{4}{*}{ AN-107 } & \multirow[t]{4}{*}{$1,050,900$} & \multirow[t]{4}{*}{ SC/SL-LIQ } & C-0 & C-NA & C-NA & \multirow[t]{4}{*}{126} & \multirow[t]{4}{*}{ NBD } \\
\hline & & & L- 107,490 & L-1.27E-07 & $\mathrm{L}-86.77$ & & \\
\hline & & & SS-33,000 & SS-1.67E-07 & SS-91.77 & & \\
\hline & & & IS-0 & IS-NA & IS-NA & & \\
\hline \multirow[t]{4}{*}{$\mathrm{AY}-101$} & \multirow[t]{4}{*}{$1,107,110$} & \multirow[t]{4}{*}{ SL-NL } & C- 14,710 & C-8.78E-08 & C-84.3 & \multirow[t]{4}{*}{485} & \multirow[t]{4}{*}{$\overline{B D}$} \\
\hline & & & L-76,560 & $\mathrm{L}-1.36 \mathrm{E}-07$ & L-98.01 & & \\
\hline & & & SS-56,680 & SS-1.49E-07 & SS-106.13 & & \\
\hline & & & IS-0 & IS-NA & IS-NA & & \\
\hline
\end{tabular}

NA - Not applicable

(a) Volumes shown are approximate.

(b)Taken from RESOLVE! Version 2.5 database (Barton et al. 1998)

SL - slurry

$\mathrm{NL}$ - no liquid

$\mathrm{SC}$ - salt cake

SS - salt sludge

LIQ - liquid

(c) Volumes shown are approximate. $\mathrm{lbm} / \mathrm{cuft}=$ pound moles per cubic foot

C - crust layer

$\mathrm{L}$ - liquid layer

SS - settled solids layer

IS - immobile solids layer

(d)GRE - Gas release event

BD - Buoyant displacement

NBD - Non-buoyant displacement 


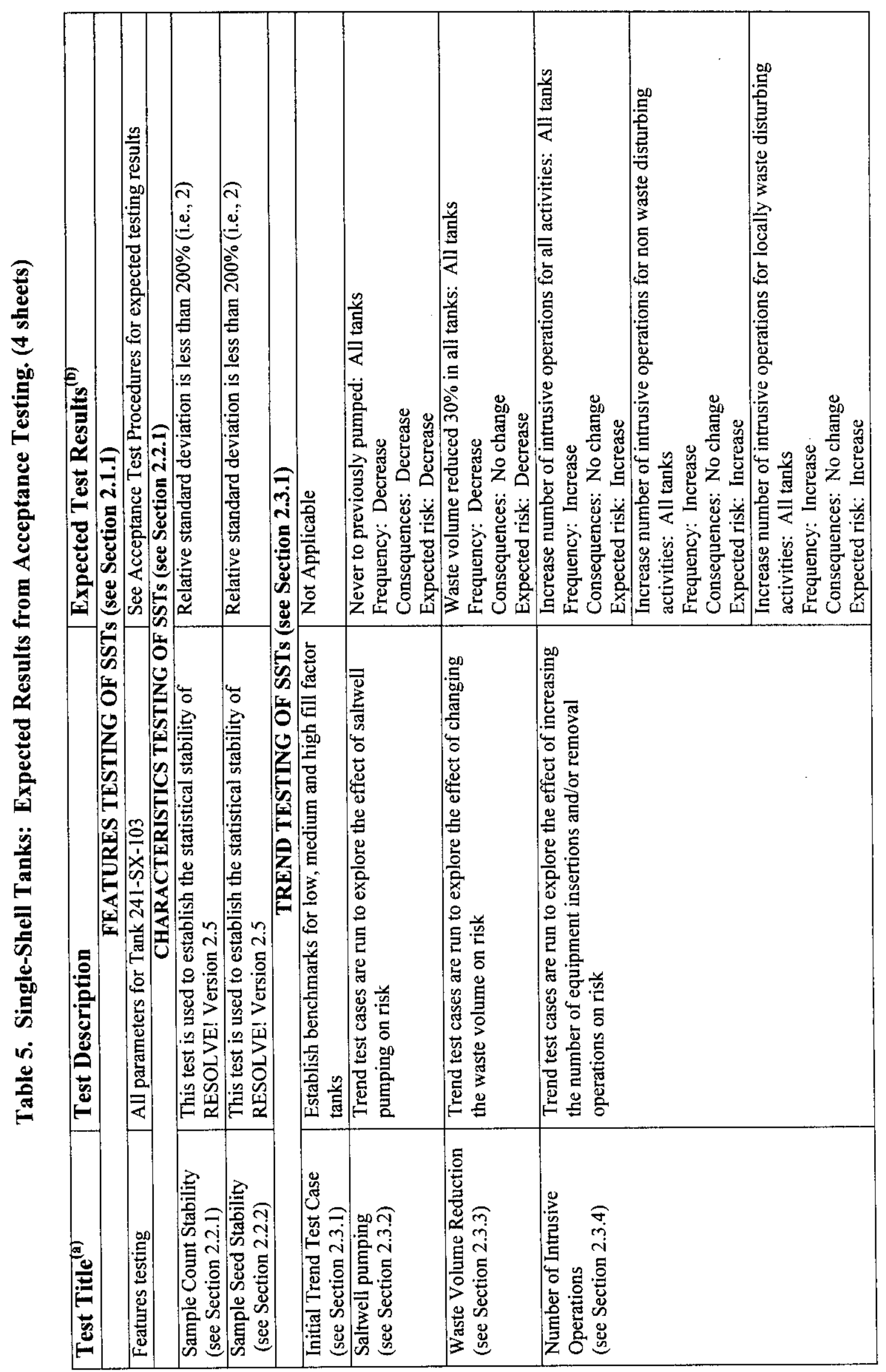


RPP-6888 REV 0

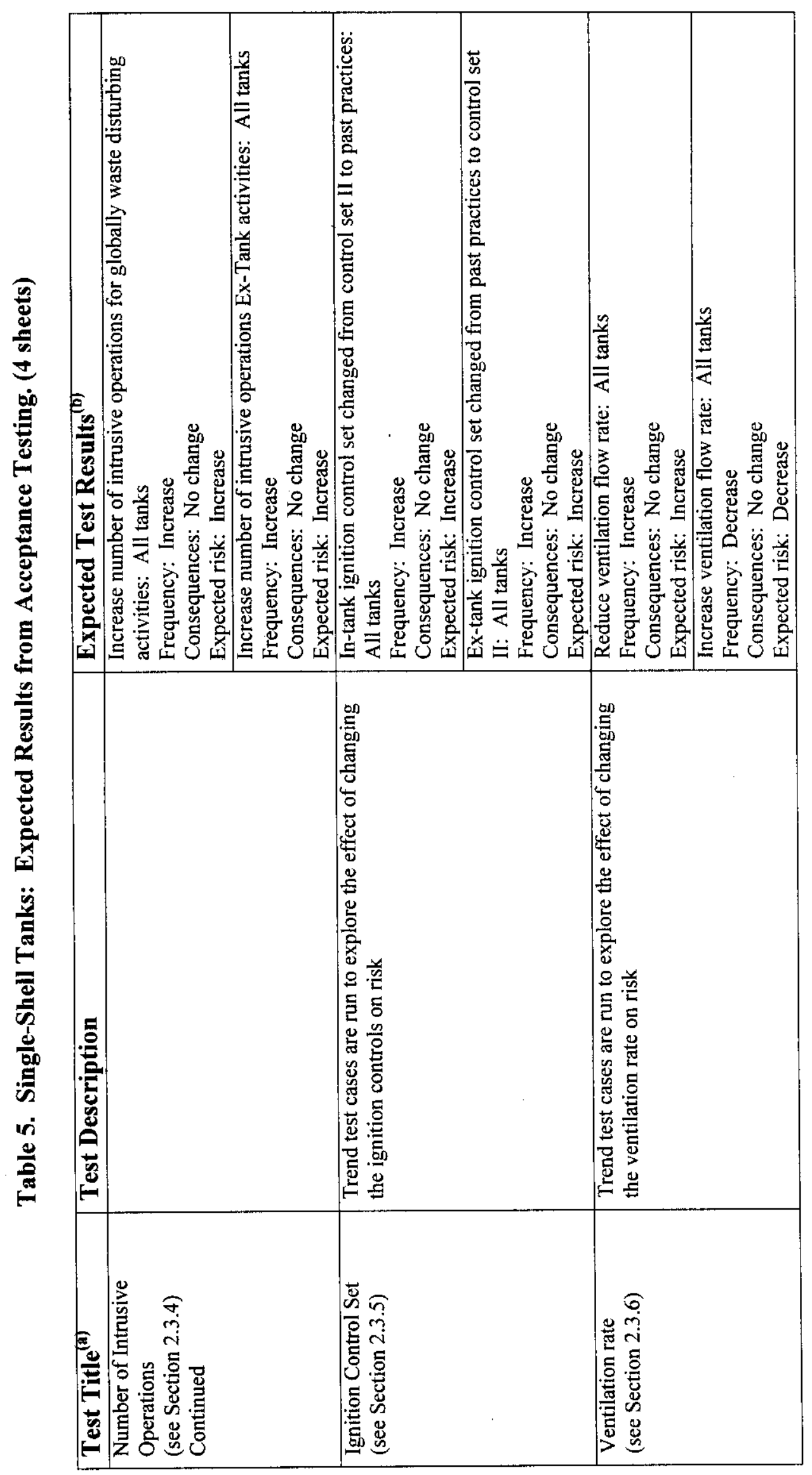


RPP-6888 REV 0

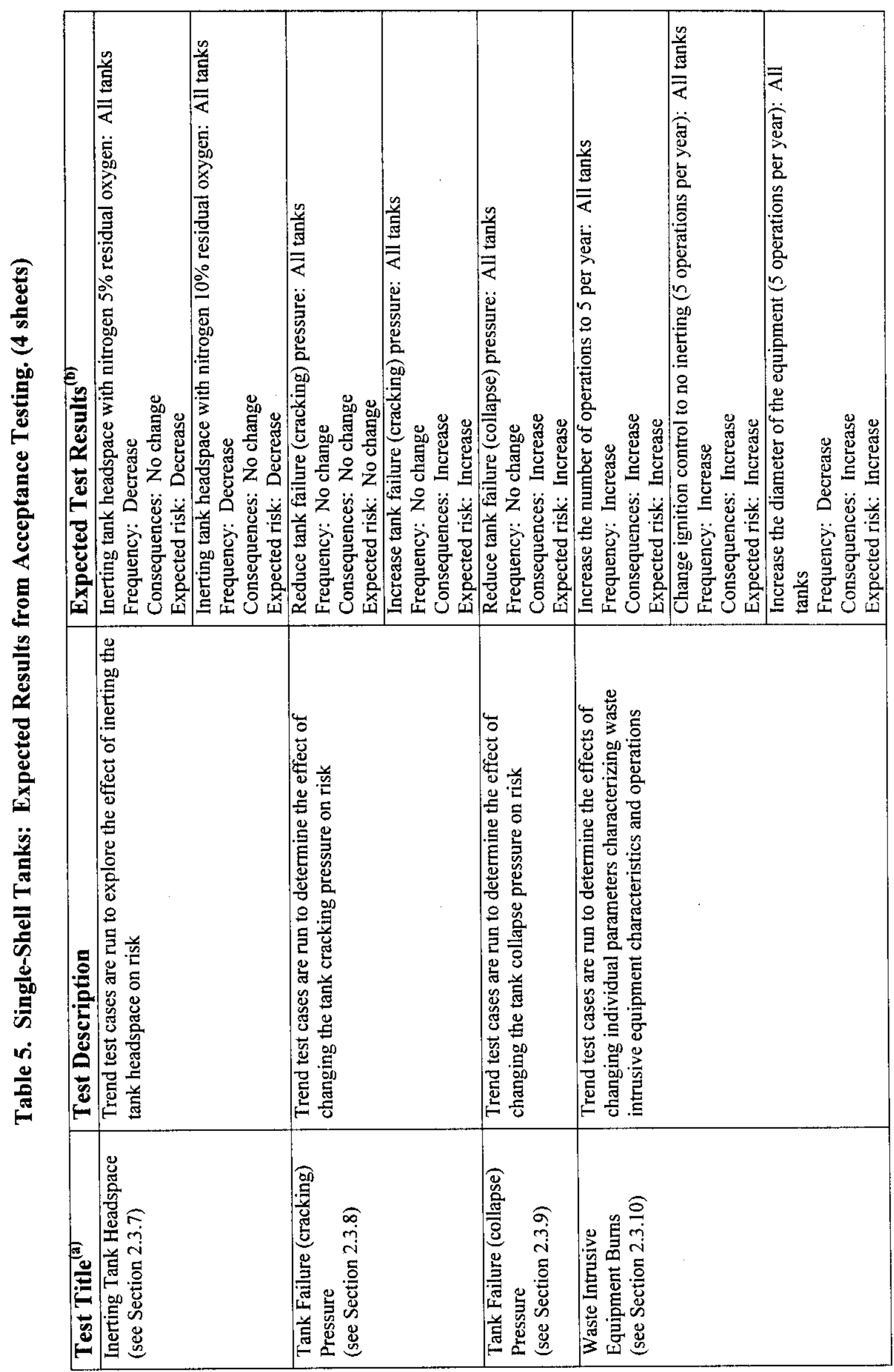


RPP-6888 REV 0

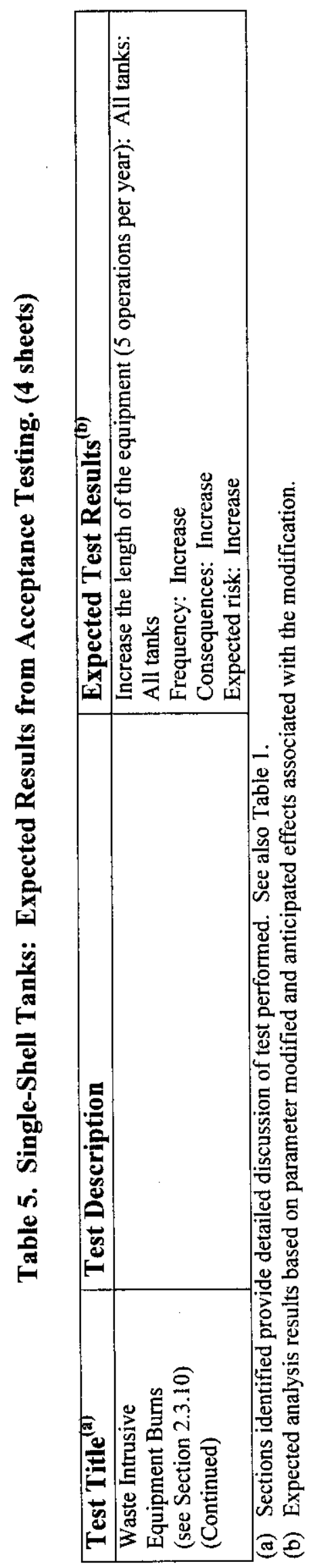


RPP-6888 REV 0

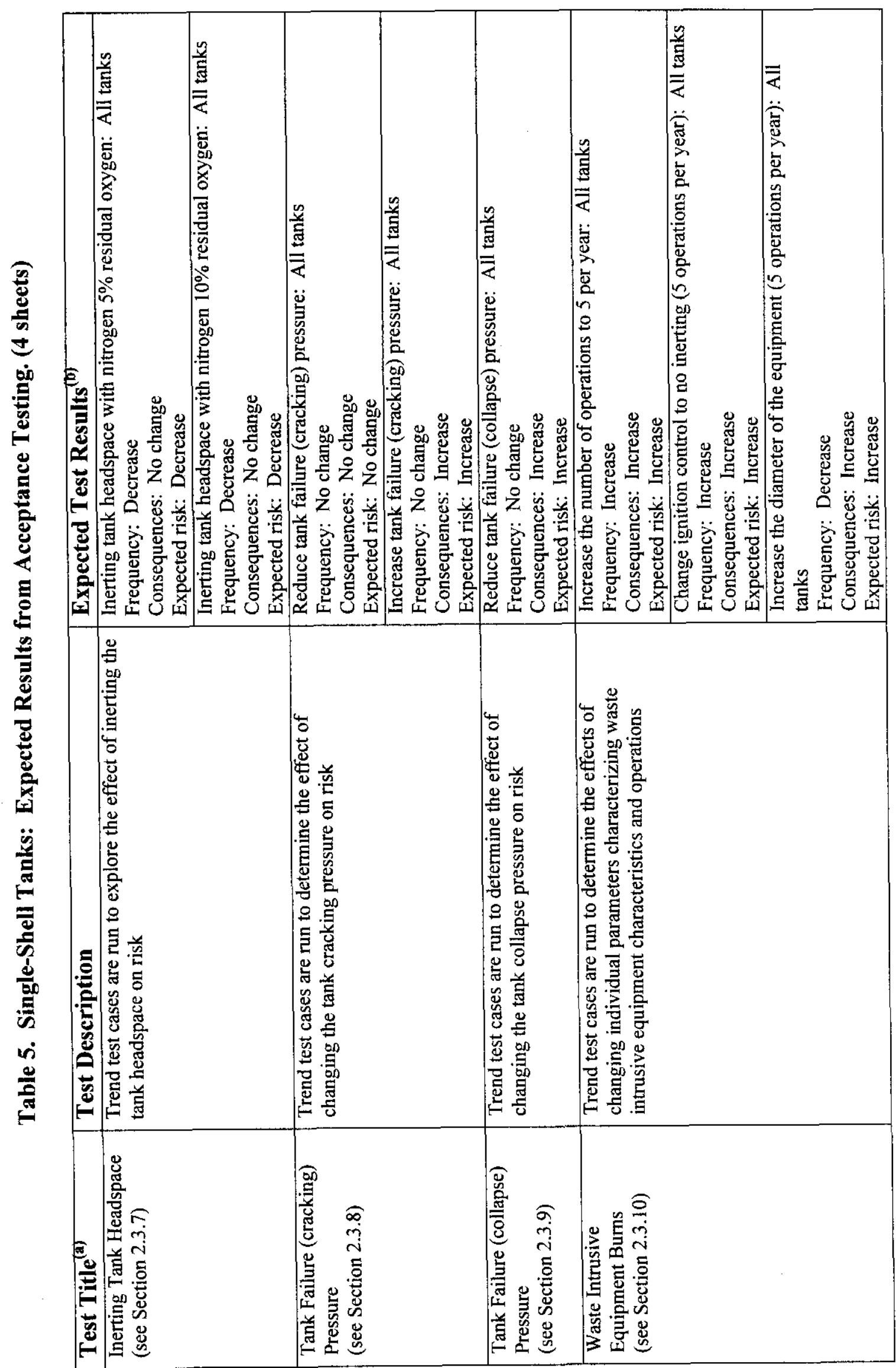


RPP-6888 REV 0

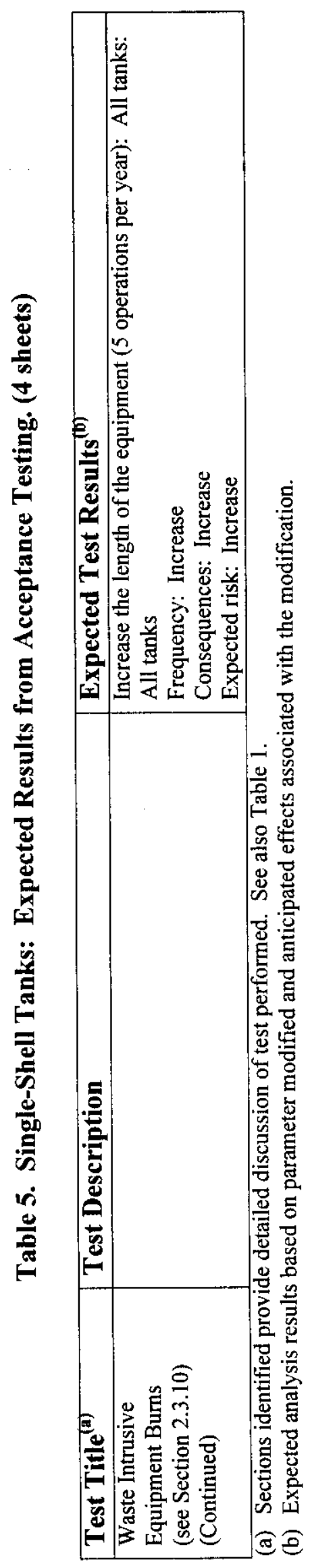




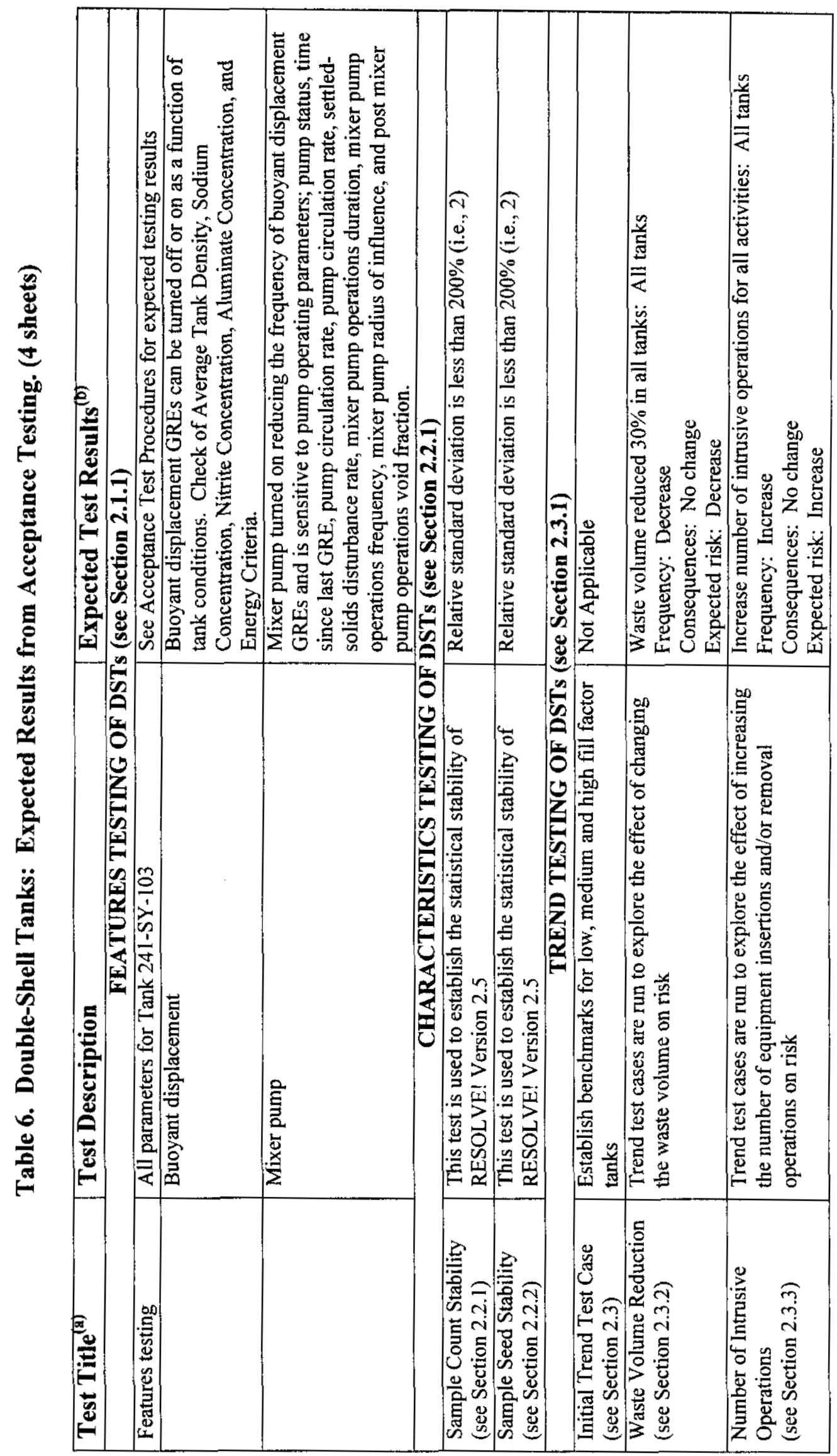


RPP-6888 REV 0

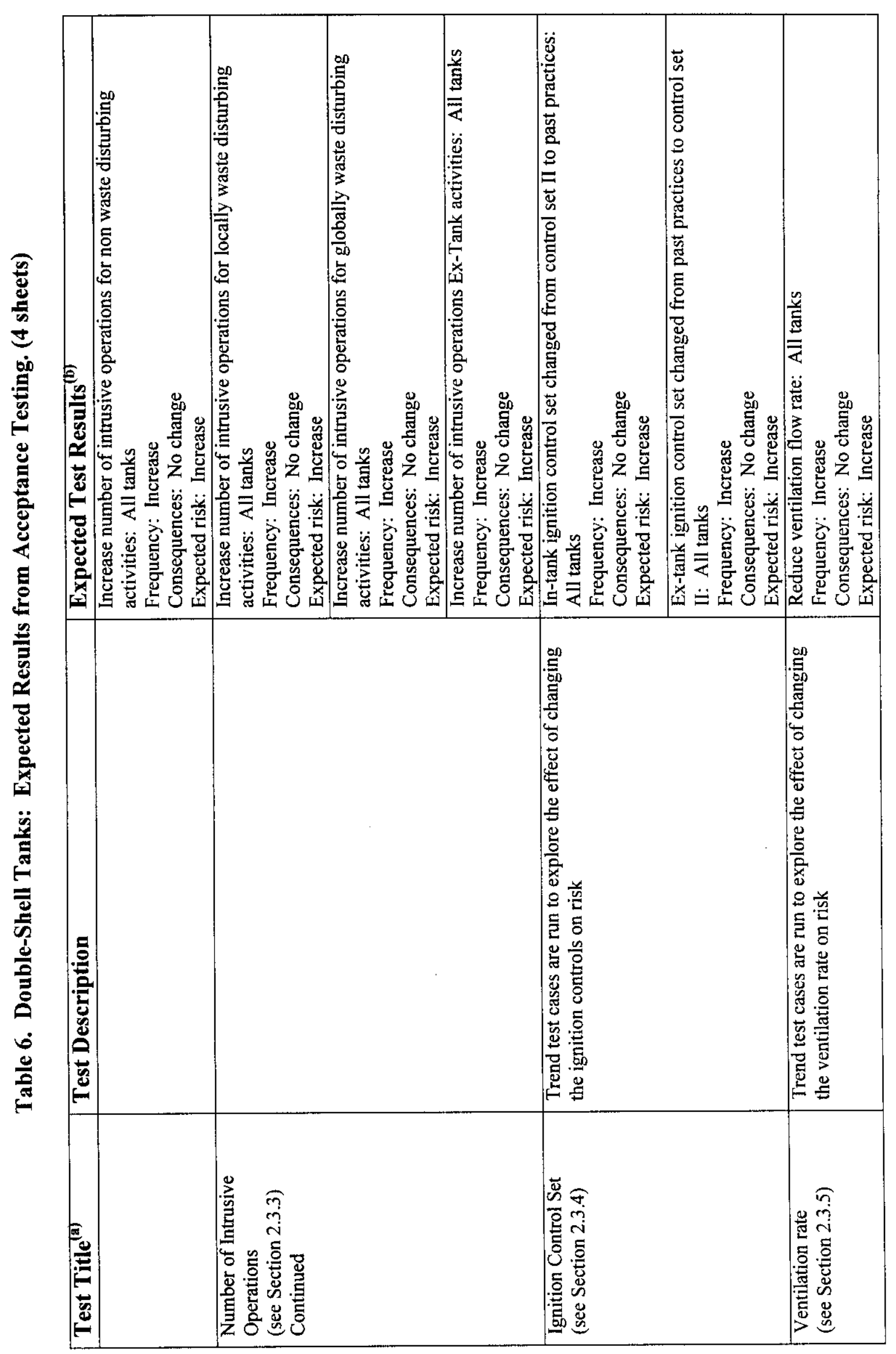


RPP-6888 REV 0

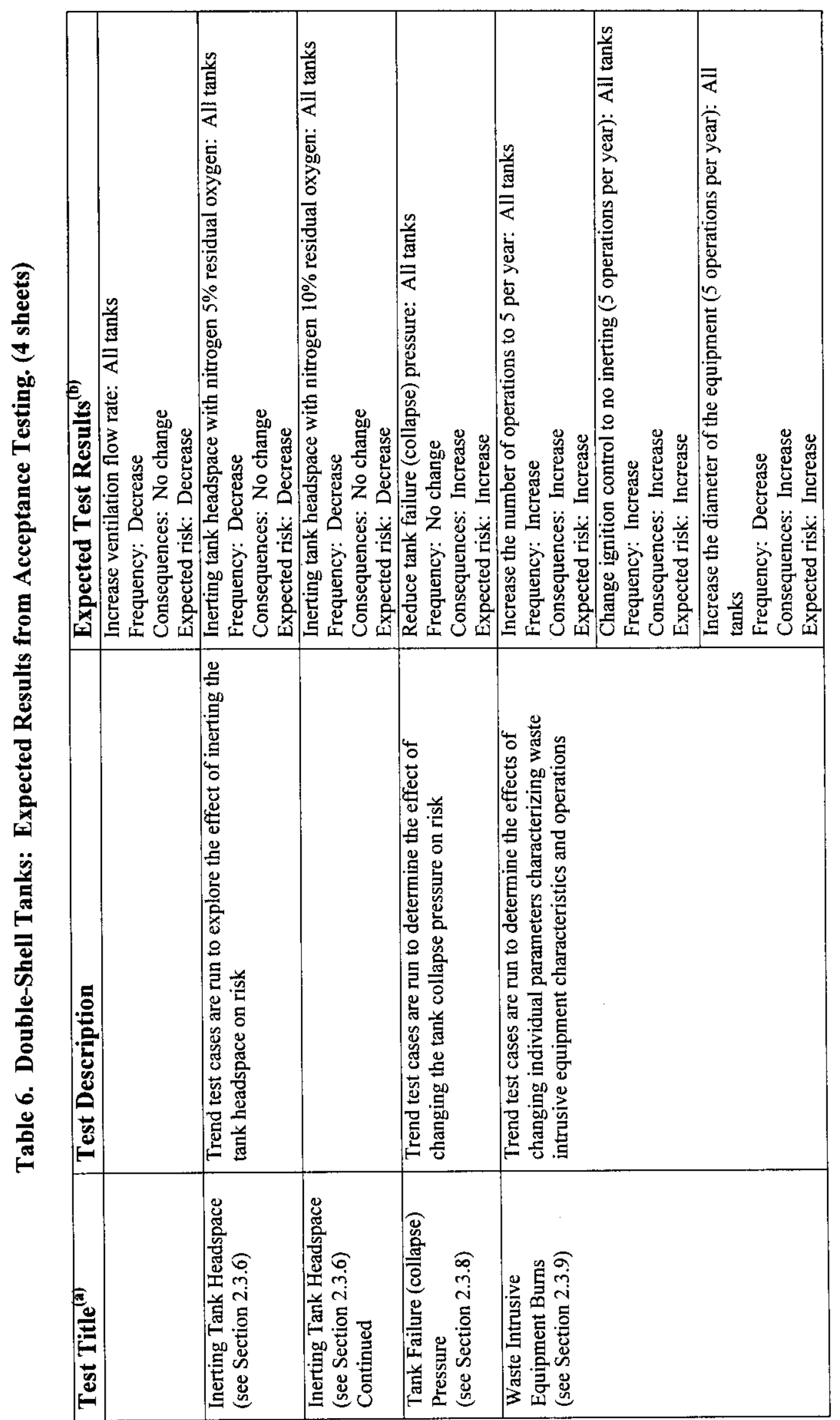


RPP-6888 REV 0

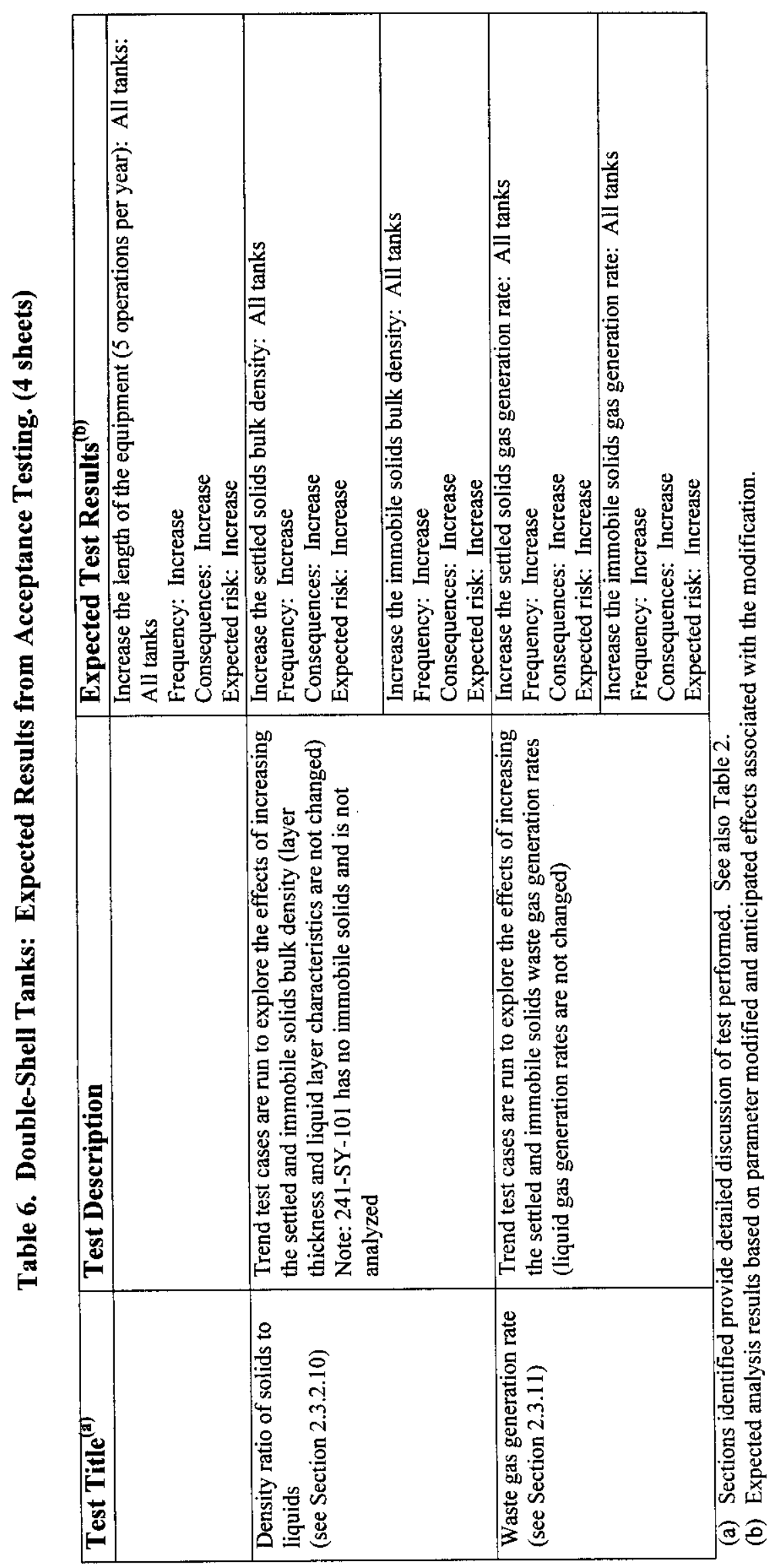


results specific results (e.g., frequency, deflagrations, detonations) are compared for each trend test to the benchmark case results.

\subsubsection{Saltwell Pumping}

This test applies to SSTs only, since only SSTs are saltwell pumped. Trend testing cases are run to explore the effect of saltwell pumping on risk. Only the saltwell pumping status is varied. The waste volume, which would change if a tank were actually saltwell pumped, is left unchanged. The influence of saltwell pumping on gas retention characteristics is an elicited parameter (Slezak and Bratzel 1997). Tanks identified as "never pumped" are changed to "previously pumped" and vice versa. Changing the saltwell pumping status from "never pumped" to "previously pumped" is expected to decrease risk. Changing the status to "previously pumped" changes the waste void fraction and GRE frequency elicitation. The end result should be a reduction in the number of flammable events, frequency, and magnitude of radiological and toxicological consequences. Table 1 provides a listing of the input parameters and values that are changed for each test.

\subsubsection{Waste Volume}

This test applies to SSTs and DSTs. Trend testing cases were run to explore the effect of changing the waste volume on risk. The waste volume is changed significantly. A reduction in the waste volume will increase the headspace volume. This effect may impact the ability of the headspace gases to reach the lower flammability limit. To test the effect of reducing waste volume, 30 percent of the existing waste was removed. Reducing the waste volume decreases the retained gas volume by providing less waste in which the gas can be stored. In addition, reducing the waste volume increases the headspace volume in which to dilute the GRE gases. Therefore, the size of the GREs and the fraction of the GREs that produce flammable conditions would decrease which would result in a decrease in the number of events. Due to the properties of the waste the impacts on the frequency and consequences are indeterminate. See Tables 1 and 2 for SSTs and DSTs respectively, for test input parameters. The total DST volume is reduced but the reduction is reflected in liquids only.

\subsubsection{Number of Intrusive Operations}

This test applies to SSTs and DSTs. Trend testing cases are run to explore the effect of increasing the number of equipment insertion and/or removal operations on risk. The number of operations is increased by a factor of five over the default value. It is expected that the increase in the number of operations increases the risk by increasing the frequency of induced GREs and the frequency of ignition sources. Only the frequency is expected to increase. There should be no change in the number of flammable events and the radiological and toxicological consequences. See Tables 1 and 2 for SSTs and DSTs, respectively, for test input parameters. 


\subsubsection{Ignition Control Set}

This test applies to SSTs and DSTs. Trend testing cases were run to explore the effect of changing the ignition controls on risk. It is expected that frequency increases as the level of ignition control is decreased from "Control Set 1" or "Control Set 2" (HNF-1999) to "Past Practices" (i.e., no controls). The ignition control sets, "Control Set 1", "Control Set 2" and "Past Practices" are described in Appendix B of Slezak and Bratzel (1997). Because the controls do affect the GREs, the controls are expected to affect the frequency but have little effect on the radiological and toxicological consequences. It is expected that changing controls from Ignition Control Set 2 to Past Practices should not change the number of flammable events or consequences; however, the frequency should increase. See Tables 1 and 2 for SST and DST, respectively, for test input parameters.

\subsubsection{Ventilation Rate}

This test applies to SSTs and DSTs. Trend testing cases were run to explore the effect on risk due to changes the ventilation flow rate (i.e., increasing or decreasing). This test involves reducing or increasing the default ventilation flow rate by a factor of 10 . It is expected that actively ventilating a tank, compared to passive ventilation could reduce the time at risk and the computed burn pressures, and perhaps even eliminate flammable conditions (Slezak and Bratzel 1997). Conversely reducing the flow rate could increase the time at risk and the computed burn pressure. Therefore, the expected result of increasing the ventilation rate is a decrease in risk, and decreasing the ventilation rate is an increase in risk. As the ventilation flow rate is modified, gases may be swept out of the tank (increased flow rate) or allowed to accumulate in the headspace (decreased flow rate). See Tables 1 and 2 for SSTs and DSTs, respectively, for test input parameters

\subsubsection{Inerting the Tank Headspace with Nitrogen}

This test applies to SSTs and DSTs. Trend testing cases were run to determine the impact of inerting the headspace. Sufficient inerting of the headspace will increase the size of GREs needed to reach flammability and can prevent combustion of mixtures that are flammable before release (Slezak and Bratzel 1997). Inerting the headspace reduces the oxidizer for the burn typically furnished by air. Inerting the tank headspace should reduce GRE flammable event frequencies and should result in less damage to the tank as well as reducing the dose consequences. Risk is expected to decrease as inerting is applied. The number of hits, as well as the frequency of burns, should decrease with a small reduction in the consequences. See Tables 1 and 2 for SSTs and DSTs, respectively, for test input parameters. 


\subsubsection{Tank Failure (Cracking) Pressure}

This test applies to SSTs only. Trend testing cases were run to explore the effect on risk of decreasing or increasing the dome cracking pressure. For this test, the dome cracking pressure is decreased or increased by $50 \%$. Structural capacity and failure evaluations are discussed in Slezak and Bratzel 1997. It is expected that risk will increase as failure pressure is reduced, and conversely decrease as failure pressure is increased. A decrease or increase in the cracking pressure from the default value should, respectively, increase or decrease the consequences but should not affect the frequency. Additionally, the number of flammable events for an increase or decrease in the cracking pressure should not change. For a decrease in the cracking pressure, more material would be released to the environment for lower combustion pressures. Conversely for the same combustion pressures, less material would be released to the environment, thus resulting in lower consequences. See Table 1 for test input parameters.

\subsubsection{Tank Failure (Collapse) Pressure}

This test applies to SSTs and DSTs. Trend testing cases were run to explore the effect on risk of decreasing the dome collapse pressure. The dome collapse pressure is reduced to one-half of the default value. Structural capacities and failure evaluations are discussed in Slezak and Bratzel 1997. It is expected that risk will increase as failure pressure is reduced. Reducing the dome collapse pressure should result in the same conclusions reached in the Tank Failure (Cracking) pressure test (see Section 2.3.7); i.e., the consequences should increase but the frequency should not be affected. Additionally, the number of flammable events for an increase or decrease in the cracking pressure should not change. The risk would therefore increase somewhat. More material would be released to the environment for lower combustion pressures. See Tables 1 and 2 for SSTs and DSTs, respectively, for test input parameters.

\subsubsection{Waste Intrusive Equipment}

This test applies to SSTs and DSTs. Trend testing cases were run to explore the effect on risk of waste intrusive equipment flammable events. A series of four tests are performed: 1) increase the number of operations per year; 2) change the ignition control from purged to not purged; 3) increase the diameter of the equipment; and 4) increase the length of the equipment. There are no waste intrusive operations in the benchmark case or initial condition. Thus increasing the number of operations will increase risk. The current ignition control requires purging waste intrusive equipment in accordance with the National Fire Prevention Association (NFPA); therefore, not purging the equipment (maintaining operations at five per year) will increase the risk over benchmark conditions and Test 1 in this series. Tests 3 and 4 of the series should affect the detonation cell size, thus an impact is expected on the number of flammable events. However, realistic changes in the equipment design (e.g., diameter and length) may be insufficient to impact the results of Test 1 in this series. Therefore the expected changes at this time are indeterminate. 


\subsubsection{Increase in Bulk Density Ratio of Solids to Liquids}

This test applies to DSTs only. Two tests are performed to determine the relative impacts of increasing the bulk density of the settled solids and immobile solids with respect to GRE behavior. These tests, unlike the previous tests, do not model operations or potential controls that would be implemented in the tank farms. However, based on the behavior of the waste it is anticipated that GRE behavior will be affected. That is, the higher the densities the more gas that will be retained in the waste and released spontaneously or due to some initiating event such as a seismic event or intrusive operation. Thus it is anticipated that the risk will increase.

\subsubsection{Increase in Waste Gas Generation Rate}

This test applies to DSTs only. Two tests are performed to determine the relative impacts of increasing the gas generation rate in the settled solids and immobile solids with respect to GRE behavior. These tests, unlike the previous tests, do not model operations or potential controls that would be implemented in the tank farms. It is anticipated that the risk will increase relatively proportional to the increased rates.

\subsubsection{Mixer Pump}

This test applies to DSTs only. The tests, in addition to the Features Tests, were performed to determine the impacts of a mixer pump on consequences. Based on operational experience it is well understood that the mixer pump will reduce the efficiency of the GREs, thus the consequences should decrease. Similarly, the frequency of buoyant displacement GREs will also decrease.

\subsubsection{Waste Transfers}

This test was performed to evaluate the AT's ability to characterize the risk associated with waste transfer operations. Both cases studied were SST to DST waste transfers. The first case modeled was a direct liquid waste transfer while the second was a water-diluted solid waste transfer. For this testing it is assumed that blending is complete and values for the layers are uniform throughout the layers, all liquids are saturated and the dilution water has no contribution to the waste compositions. Table 7 lists the waste transfer input parameters that were tested. 


\section{RPP-6888 REV 0}

Table 7. Waste Transfer Input Parameters.

\begin{tabular}{|c|c|c|c|c|c|c|c|c|}
\hline & \multicolumn{4}{|c|}{ Case 1 } & \multicolumn{4}{c|}{ Case 2 } \\
\cline { 2 - 9 } & Sending Tank A-101 & \multicolumn{2}{|c|}{$\begin{array}{c}\text { Receiving Tank } \\
\text { AN-101 }\end{array}$} & \multicolumn{2}{|c|}{$\begin{array}{c}\text { Sending Tank } \\
\text { A-101 }\end{array}$} & \multicolumn{2}{|c|}{$\begin{array}{c}\text { Receiving Tank } \\
\text { AY-101 }\end{array}$} \\
\cline { 2 - 9 } & gal & $\left.\mathbf{( f t}^{3}\right)$ & gal & $\left.\mathbf{( f t}^{3}\right)$ & gal & $\left.\mathbf{( f t}^{3}\right)$ & gal & $\left.\mathbf{( f t}^{3}\right)$ \\
\hline $\begin{array}{c}\text { Total volume } \\
\text { initial }\end{array}$ & 953,133 & $(127,415)$ & 160,088 & $(21,401)$ & 295,080 & $(39,446)$ & 154,012 & $(20,588)$ \\
\hline $\begin{array}{c}\text { Crust Volume } \\
\text { Transferred }\end{array}$ & - & - & - & - & - & - & - & - \\
\hline $\begin{array}{c}\text { Liquid } \\
\text { Volume } \\
\text { Transferred }\end{array}$ & 200,000 & $(26,736)$ & 200,000 & $(26,736)$ & - & - & - & - \\
\hline $\begin{array}{c}\text { Solids } \\
\text { Volume } \\
\text { Transferred }\end{array}$ & - & - & - & - & 270,000 & $(36,094)$ & 270,000 & $(36,094)$ \\
\hline $\begin{array}{c}\text { Immobile } \\
\text { Solids } \\
\text { Volume } \\
\text { Transferred }\end{array}$ & - & - & - & - & - & - & - & - \\
\hline $\begin{array}{c}\text { Dilution } \\
\text { Volume }\end{array}$ & - & - & - & - & - & - & 270,000 & $(36,094)$ \\
\hline $\begin{array}{c}\text { Total volume } \\
\text { final }\end{array}$ & 753,133 & $(100,679)$ & 360,088 & $(48,137)$ & 25,080 & $(3,353)$ & 694,012 & $(92,776)$ \\
\hline $\begin{array}{c}\text { Pumping Rate } \\
\text { (per min) }\end{array}$ & 72.94 & $(9.75)$ & 72.94 & $(9.75)$ & 72.94 & $(9.75)$ & 72.94 & $(9.75)$ \\
\hline
\end{tabular}




\section{RPP-6888 REV 0}

This page intentionally left blank. 


\subsection{SUMMARY OF TEST RESULTS}

The results and conclusions reached regarding the acceptability of RESOLVE! Version 2.5 and the insights gained during testing are summarized in the following Sections. Appendices A and B provide the detailed results. Section 4.0 discusses any limitations identified during testing.

\subsection{FEATURES TESTING}

As discussed in Section 2.1, an extensive test of the AT and GUI features is discussed in Attachment 1. The features testing performed in this acceptance test was limited to the options selected during characteristics and trend testing. This limited set of features tests, based on the parameter modifications, will also verify that the code and data libraries are consistent with the changes in the baseline documentation due to comment incorporation from the independent review.

Additional Features Tests were performed for the DSTs, including turning on and off the mixer pump, buoyant displacement GRE go/no-go tests, waste transfers, and waste intrusive equipment impacts. As stated previously stated, significant developmental testing was performed. As a result of this testing all features performed as expected.

\subsection{CHARACTERISTICS TESTS}

The following table (Table 8) summarizes the results of the Characteristics Tests performed. As can be seen, with the exception of the DST sample count test, all results meet the established criteria discussed in Section 2.2.

The calculated mean toxicological sum of fractions for a sample count of 950 was approximately eight times the highest calculated sum of fractions for the other tests (i.e., $247 \mathrm{vs} \mathrm{31).} \mathrm{All} \mathrm{other}$ calculated values, as well as the $95^{\text {th }}$ percentile value for sample count equal to 950 , are within the minimum and maximum values. Therefore, prior to reporting toxicological consequences multiple runs should be performed using different seeds and sample sizes (or counts) and evaluated for appropriateness.

\subsection{SUMMARY OF TREND TESTING RESULTS}

Figures 1 through 32 provide a surnmary of the trend testing results by tank for each of the parameters modified. Appendices A and B provide the detailed data and quantitative comparisons of the calculated results for the parameter modifications to the benchmark case. Consequences were not calculated for tank Tank 241-T-203, thus no graphical representations are provided for the inerting cases. 
RPP-6888 REV 0

Table 8. Summary of the Results of the Characteristics Tests Performed.

\begin{tabular}{|c|c|c|c|c|c|}
\hline \multirow{2}{*}{$\begin{array}{c}\text { Characteristic } \\
\text { Test }\end{array}$} & \multicolumn{5}{|c|}{ Test Results - Relative Standard Deviation (\%) } \\
\hline & $\begin{array}{c}\text { Number of } \\
\text { Events } \\
\text { Modeled }\end{array}$ & $\begin{array}{l}\text { Accident } \\
\text { Frequency }\end{array}$ & $\begin{array}{c}\text { Radiological } \\
\text { Consequences }\end{array}$ & $\begin{array}{l}\text { Toxicological } \\
\text { Consequences }\end{array}$ & $\begin{array}{l}\text { Expected } \\
\text { Risk }\end{array}$ \\
\hline \multicolumn{6}{|c|}{ SSTs $-241-S-102$} \\
\hline $\begin{array}{l}\text { Sample Count } \\
\text { Sensitivity }\end{array}$ & 43.49 & 6.5 & 59.78 & 19.99 & 96.63 \\
\hline $\begin{array}{l}\text { Sample Seed } \\
\text { Sensitivity }\end{array}$ & 4.98 & 7.26 & 44.94 & 19.03 & 51.68 \\
\hline \multicolumn{6}{|c|}{ DST - 241-AW-102 (Non-BD) } \\
\hline $\begin{array}{l}\text { Sample Count } \\
\text { Sensitivity }\end{array}$ & 42.12 & 62.76 & 102.24 & 251.55 & 161.43 \\
\hline $\begin{array}{l}\text { Sample Seed } \\
\text { Sensitivity }\end{array}$ & 0.18 & 70.57 & 127.30 & 96.02 & 143.39 \\
\hline \multicolumn{6}{|c|}{ DST - 241-AN-103 (BD) } \\
\hline $\begin{array}{l}\text { Sample Count } \\
\text { Sensitivity }\end{array}$ & 42.24 & 27.55 & 103.43 & 68.31 & 158.89 \\
\hline $\begin{array}{l}\text { Sample Sed } \\
\text { Sensitivity }\end{array}$ & 1.39 & 20.55 & 99.37 & 68.55 & 126.62 \\
\hline
\end{tabular}

BD - Buoyant displacement

As can be seen from the figures the majority of the parameters modified for both DSTs and SSTs did not significantly affect the benchmark results. This is the same conclusion reached during acceptance testing of Version 2.13 (see Attachment 1). The parameter modifications that did significantly impact the results include waste volume reduction, an increase or decrease in ventilation flow rate, and changes in the waste and waste gas generation characteristics (DST only). In each of these cases the results trend as expected.

With respect to waste intrusive equipment, as expected for both DSTs and SSTs, increasing the number of waste intrusive activities increased the number of potential deflagrations and detonations when compared to the benchmark case. Additionally as expected, removing the purge from the waste intrusive equipment significantly increased the number of potential deflagrations and detonations when compared to the base case or five operations per year. Increasing the equipment diameter or length had no impact on the base case results. Because there are no or limited observed data regarding waste intrusive equipment, the results cannot be evaluated for appropriateness.

Figure 18 graphically shows the results obtained due to modifications in the characteristics of the settled solids and the immobile solids (hard pan) in Tank 241-AN-107. This represents the greatest change form benchmark conditions for all tests performed. As can be seen increasing the ratio of the settled solids to the liquids increased the number of deflagrations in Tank 241-AN-107. Additionally, the change in waste characteristics increased the BD GRE frequency in Tank 241-AN-107. 
Not shown graphically are the results obtained from buoyant displacement, mixer pump, and waste transfers testing. As expected changes in the five criteria identified in Table 6 (DST Features Testing) created buoyant displacement GREs in non-buoyant displacement tanks and vice versa. Similarly as expected, the calculated results from turning on the mixer pump and changing selected parameters to model existing conditions in Tank 241-SY-101 were validated to current conditions or observed GRE behavior in the tank. The waste transfer test described in Section 2.1.13 performed as expected;s, post transfer sending and receiving tank analysis results were comparable to tank analyses. 
RPP-6888 REV 0

This page intentionally left blank. 


\subsection{LIMITATIONS}

This section discusses the limitations identified as a result of acceptance testing. There were two limitations identified. One resulted from "Characteristics Testing" and the other, "Waste Intrusive Equipment" was identified based on a lack of actual or observed data to validate the results. Each of the limitations is discussed in the following.

\subsection{CHARACTERISTICS TESTING}

As discussed in Section 3.2, with the exception of the DST sample count test, all results met the established criteria discussed in Section 2.2. The calculated mean toxicological sum of fractions exceeded the acceptance relative standard deviation criteria. The calculated sum of fractions for one sample count, 950, was approximately 8 times the highest calculated sum of fractions for the other tests (i.e., 247 vs 31 ). All other calculated values, as well as the $95^{\text {th }}$ percentile value for sample count equal to 950 , are within the minimum and maximum values. Therefore, prior to reporting toxicological consequences multiple runs should be performed using different seeds and sample sizes (or counts) and evaluated for appropriatness.

\subsection{WASTE INTRUSIVE EQUIPMENT}

Based on a lack of actual or observed data, the results from testing parameter modifications in the operations of waste intrusive equipment and equipment design could not be validated. However, the results from the modifications trended as expected (see Section 3.3). This limitation was also identified during verification and validation testing of Version 2.5 (Cheng et al. 2000). Therefore, when reporting the results associated with waste intrusive equipment, it should be noted that the results cannot be validated and should be used for comparison purposes only. 


\section{RPP-6888 REV 0}

This page intentionally left blank 


\section{RPP-6888 REV 0}

\subsection{REFRENCES}

Barton, W. B., 1998, Gas Release Event Safety Analysis Tool Pedigree Database for Hanford Tanks, HNF-SD-WM-TI-806, Rev. 3, Lockheed Martin Hanford Corporation, Richland, Washington.

Cheng, W., B. Lewis, A. L. Hodges, S. E. Slezak, P. A. Trellue, S. A. Barker, J. C. Lavender, S. G. Ashbaugh, W. M. Guerra, J. Kingson, L. F. McGlinchy, and A. Riddle, 2000, Refined Safety Analysis Tool Software Verification and Validation Report: Resolve Version 2.5, RPP-6873, Rev. 0, prepared by Sandia National Laboratory for the Hanford River Protection Project, CH2M HILL Hanford Group, Inc., Richland, Washington.

HNF, 1999, Tank Waste Remediation System Final Safety Analysis Report,

HNF-SD-WM-SAR-067, Rev. 0, Fluor Daniel Hanford, Richland, Washington.

Lavender, J. C., B. D. Lacey, and A. B. Webb, 1998, Letter Report: RESOLVE! Version 1.51 Acceptance Testing: Test Plan and Test Results, Pacific Northwest National Laboratory, Richland, Washington.

Slezak, S. E., F. Gelbard, and W. Cheng, 1999, Implementation Details of the Flammable Gas Refined Safety Analysis Methodology, Sandia National Laboratories, Albuquerque, New Mexico.

Slezak, S. E., D. C. Williams, W. Cheng, and D. R. Bratzel, 2000, Refined Safety Analysis Methodology for Flammable Gas Risk Assessment in Hanford Site Tanks, HNF-SD-WM-ES-410, Rev. 3, prepared by Sandia National Laboratory for CH2M HILL Hanford Group, Inc., Richland, Washington. 
RPP-6888 REV 0

This page intentionally left blank. 
RPP-6888 REV 0

APPENDIX A

\section{SST ACCEPTANCE TEST RESULTS}


RPP-6888 REV 0

This page left intentionally blank. 


\section{APPENDIX A}

This Appendix provides the results of the SST acceptance testing. A strict comparison of the benchmark results to the trend analysis results does not consider the magnitude of the difference. For example, if the benchmark mean consequence value is $4.567 \mathrm{E}-05 \mathrm{~Sv}$ and the mean trend test analysis result is $4.566 \mathrm{E}-05 \mathrm{~Sv}$, a comparison of this type would indicate that by modifying a specific parameter, the consequences decreased; however, based on the conservatisms and uncertainties incorporated in the Analysis Tool, a better conclusion would be that there is no change. 


\section{RPP-6888 REV 0}

This page intentionally left blank. 
Table A-1. SST Acceptance Test Results: GRE Behavior. (Seed: 90,000,000, Sample Count: 1000)

\begin{tabular}{|c|c|c|c|c|c|c|c|c|c|c|c|c|c|}
\hline \multirow[t]{2}{*}{ Tank } & \multirow{2}{*}{$\begin{array}{c}\text { GRE } \\
\text { Behavior }\end{array}$} & \multirow{2}{*}{$\begin{array}{c}\text { Number of } \\
\text { Events }\end{array}$} & \multirow{2}{*}{\multicolumn{3}{|c|}{$\begin{array}{c}\text { Number of } \\
\text { Deflagrations }\end{array}$}} & \multirow{2}{*}{$\begin{array}{l}\text { Number of } \\
\text { Detonations }\end{array}$} & & \multicolumn{4}{|c|}{ \%LFL } & \multirow{2}{*}{\multicolumn{2}{|c|}{$\begin{array}{c}\text { Mean } \\
\text { Number of } \\
\text { Events/year }\end{array}$}} \\
\hline & & & & & & & & $\begin{array}{c}\text { Mean } \\
\text { Frequency }\end{array}$ & & $\begin{array}{c}\text { Mean } \\
\text { Concentration }\end{array}$ & & & \\
\hline \multicolumn{14}{|c|}{ BENCHMARK } \\
\hline \multirow[t]{4}{*}{$A-102$} & all sizes & 11962 & & 272 & & 0 & & $9.28 \mathrm{E}-03$ & & 18.70 & & $7.91 \mathrm{E}-02$ & \\
\hline & small & 3986 & & 0 & & 0 & & $2.21 \mathrm{E}-02$ & & 0.07 & & $6.68 \mathrm{E}-02$ & \\
\hline & medium & 3988 & & 272 & & 0 & & $4.42 \mathrm{E}-03$ & & 54.40 & & $9.37 \mathrm{E}-03$ & \\
\hline & large & 3988 & & 0 & & 0 & & $1.3 \mathrm{IE}-03$ & & 1.72 & & $2.69 \mathrm{E}-03$ & \\
\hline \multirow[t]{4}{*}{$\mathrm{B}-111$} & all sizes & 11828 & & 116 & & 0 & & $6.44 \mathrm{E}-02$ & & 10,80 & & $5.93 \mathrm{E}-01$ & \\
\hline & small & 3940 & & 0 & & 0 & & I.62E-01 & & 0.12 & & $5.22 \mathrm{E}-01$ & \\
\hline & medium & 3944 & & 116 & & 0 & & $2.44 \mathrm{E}-02$ & & 29.20 & & $5.44 \mathrm{E}-02$ & \\
\hline & large & 3944 & & 0 & & 0 & & $7.08 \mathrm{E}-03$ & & 3.08 & & $1.50 \mathrm{E}-02$ & \\
\hline \multirow[t]{4}{*}{$S-102$} & all sizes & 12945 & & 1752 & & 0 & & $6.50 \mathrm{E}-03$ & & 154.00 & & $5.53 \mathrm{E}-02$ & \\
\hline & small & 4313 & & 0 & & 0 & & $1.52 \mathrm{E}-02$ & & 2.52 & & $4.60 \mathrm{E}-02$ & \\
\hline & medium & 4316 & & 1386 & & 0 & & $3.13 \mathrm{E}-03$ & & 397.00 & & $6.80 \mathrm{E}-03$ & \\
\hline & large & 4316 & & 366 & & 0 & & $1.18 \mathrm{E}-03$ & & 61.70 & & $2.40 \mathrm{E}-03$ & \\
\hline \multirow[t]{4}{*}{$T-203$} & all sizes & 11628 & & 110 & & 0 & & $8.95 \mathrm{E}-03$ & & 8.22 & & $7.71 \mathrm{E}-02$ & \\
\hline & small & 3876 & & 0 & & 0 & & $2.15 \mathrm{E}-02$ & & 0.66 & & $6.55 \mathrm{E}-02$ & \\
\hline & medium & 3876 & & 22 & & 0 & & $4.07 \mathrm{E}-03$ & & 7.02 & & $8.69 \mathrm{E}-03$ & \\
\hline & large & 3876 & & 88 & & 0 & & $1.32 \mathrm{E}-03$ & & 17.00 & & $2.74 \mathrm{E}-03$ & \\
\hline \multicolumn{14}{|c|}{ SALT WELL PUMPING } \\
\hline $\mathrm{A}-102$ & all sizes & 11887 & $<1$ & 264 & $<1$ & 0 & $=$ & $9.56 \mathrm{E}-03$ & $>1$ & 17.80 & $<1$ & $8.24 \mathrm{E}-02$ & $\geq$ \\
\hline \multicolumn{14}{|c|}{ WASTE VOLUME REDUCTION } \\
\hline B-111 & all sizes & 11888 & $>$ & 76 & $<$ & 0 & $=$ & $6.65 \mathrm{E}-02$ & $>1$ & 6.53 & $<$ & $6.14 \mathrm{E}-01$ & $>$ \\
\hline \multicolumn{14}{|c|}{ INTRUSIVE OPERATIONS INCREASE ALL } \\
\hline S-102 & all sizes & 12129 & $<$ & 1672 & $<$ & 0 & $=$ & $6.64 \mathrm{E}-03$ & $>1$ & 156.00 & $>$ & $4.83 \mathrm{E}-02$ & $<$ \\
\hline & small & 4041 & $<$ & 2 & $>1$ & 0 & $=$ & $1.46 \mathrm{E}-02$ & $<$ & 3.13 & $>$ & $3.73 \mathrm{E}-02$ & $<$ \\
\hline & medium & 4044 & $<$ & 1320 & $<$ & 0 & $=$ & $4.2 \mathrm{IE}-03$ & $>$ & 403.00 & $>$ & $8.74 \mathrm{E}-03$ & $>$ \\
\hline & large & 4044 & $<$ & 350 & $<1$ & 0 & $=$ & $1.08 \mathrm{E}-03$ & $<1$ & 60.70 & $<$ & $2.18 \mathrm{E}-03$ & $<$ \\
\hline & & INTRUSIVI & $\mathbf{O P}$ & ERATIONS I & $\mathbf{N C}$ & REASE NON & WA & STE DISTU & RBI! & NG & & & \\
\hline S-102 & all sizes & 12945 & $=$ & 1752 & $\equiv$ & 0 & $=$ & $6.50 \mathrm{E}-03$ & $=$ & 154.00 & $=$ & $5.53 \mathrm{E}-02$ & $=$ \\
\hline & small & 4313 & $=$ & 0 & $=$ & 0 & $=$ & $1.52 \mathrm{E}-02$ & $=$ & 2.52 & $=$ & $4.60 \mathrm{E}-02$ & $=$ \\
\hline & medium & 4316 & $=$ & 1386 & $=$ & 0 & $=$ & $3.13 \mathrm{E}-03$ & $=1$ & 397.00 & $=$ & $6.80 \mathrm{E}-03$ & $=$ \\
\hline & large & 4316 & $=$ & 366 & $=$ & 0 & $=$ & $1.18 \mathrm{E}-03$ & $=1$ & 61.70 & $=$ & $2.40 \mathrm{E}-03$ & $=$ \\
\hline & & RUSIVE 0 & $\mathbf{E n}$ & ATIONS INC & $\mathbf{R E}$ & ASE LOCAL & LY & WASTE DIS & TUR & RBING & & & \\
\hline S-102 & all sizes & 12213 & $<1$ & 1710 & $<1$ & 0 & $=$ & $8.15 \mathrm{E}-03$ & $>1$ & 156.00 & $>$ & $6.49 \mathrm{E}-02$ & $>$ \\
\hline & small & 4069 & $<$ & 0 & $=$ & 0 & $=$ & $1.99 \mathrm{E}-02$ & $>$ & 2.55 & $>$ & $5.51 \mathrm{E}-02$ & $>$ \\
\hline & medium & 4072 & $<$ & 1338 & $<$ & 0 & $=$ & $3.64 \mathrm{E}-03$ & $>$ & 406.00 & $>$ & $7.97 \mathrm{E}-03$ & $>$ \\
\hline & large & 4072 & $<$ & 372 & $>$ & 0 & $=$ & $8.70 \mathrm{E}-04$ & $<1<+$ & 61.10 & $<$ & $1.79 \mathrm{E}-03$ & $<$ \\
\hline & & RUSIVE OI & ER & ATIONS INCI & $\mathbf{R E} t$ & SE GLOBA & $\mathbf{L Y}$ & WASTE DI & TUU & RBING & & & \\
\hline $\mathrm{S}-102$ & all sizes & 11938 & $<$ & 1648 & $<1$ & 0 & $=$ & $7.3 \mathrm{IE}-03$ & $>1$ & 153.00 & $<$ & $5.31 \mathrm{E}-02$ & $<$ \\
\hline & small & 3978 & $<$ & 4 & $>$ & 0 & $=$ & $1.68 \mathrm{E}-02$ & $>$ & 3.78 & $>$ & $4.23 \mathrm{E}-02$ & $<$ \\
\hline & medium & 3980 & $<$ & 1270 & $<$ & 0 & $=1$ & $4.28 \mathrm{E}-03$ & $>$ & 394.00 & $<$ & $8.96 \mathrm{E}-03$ & $>$ \\
\hline & large & 3980 & $<$ & 374 & $>$ & 0 & $=$ & $8.73 \mathrm{E}-04$ & $<$ & 60.90 & $<$ & $1.77 \mathrm{E}-03$ & $<$ \\
\hline & & & $\mathbf{R} \mathbf{T}$ & JSIVE OPERA & TI & ONS INCREA & ISE & EX-TANK & & & & & \\
\hline S-102 & all sizes & 12945 & $=1$ & 1752 & $=$ & 0 & $=$ & $6.50 \mathrm{E}-03$ & $\Longrightarrow$ & 154.00 & $=$ & $5.53 \mathrm{E}-02$ & $=$ \\
\hline & small & 4313 & $=$ & 0 & $=$ & 0 & $=$ & $1.52 \mathrm{E}-02$ & $=$ & 2.52 & $=$ & $4.60 \mathrm{E}-02$ & $=$ \\
\hline & medium & $\overline{4316}$ & $=$ & 1386 & $=$ & 0 & $=$ & $3.13 \mathrm{E}-03$ & $=1$ & 397.00 & $=$ & $6.80 \mathrm{E}-03$ & $=$ \\
\hline & large & 4316 & $=$ & 366 & $=$ & 0 & $=$ & $1.18 \mathrm{E}-03$ & $=$ & 61.70 & $=$ & $2.40 \mathrm{E}-03$ & $=$ \\
\hline & & $\overline{\text { INT }}$ & US & IVE OPERAT & 10 & NS INCREAS & EE & V/EX-TANK & & & & & \\
\hline S-102 & all sizes & 12129 & $<$ & 1672 & $<1$ & 0 & $=$ & $6.64 \mathrm{E}-03$ & $>$ & 156.00 & $>$ & $4.83 \mathrm{E}-02$ & < \\
\hline & small & 4041 & $<1$ & 2 & $>$ & 0 & $=$ & $1.46 \mathrm{E}-(02$ & $<+$ & 3.13 & $>$ & $3.73 \mathrm{E}-02$ & $<$ \\
\hline & medium & 4044 & $<$ & 1320 & $<$ & 0 & $=$ & $4.21 \mathrm{E}-03$ & $>$ & 403.00 & $>$ & $8.74 \mathrm{E}-03$ & $>$ \\
\hline & large & 4044 & $<$ & 350 & $<$ & 0 & $=$ & $1.08 \mathrm{E}-03$ & $<$ & 60.70 & $<$ & $2.18 \mathrm{E}-03$ & $<$ \\
\hline & & & & IGNITION C & JN & TROL SET IT & $8-1$ & INK & & & & & \\
\hline A-102 & all sizes & 11962 & $=$ & 272 & $<$ & 0 & $=$ & $9 . \overline{28 \mathrm{E}-03}$ & $>$ & 18.70 & $<$ & $7.91 \mathrm{E}-02$ & 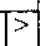 \\
\hline
\end{tabular}


Table A-1. SST Acceptance Test Results: GRE Behavior. (Seed: 90,000,000, Sample Count: 1000)

\begin{tabular}{|c|c|c|c|c|c|c|c|c|c|c|c|c|c|}
\hline \multirow[t]{2}{*}{ Tank } & \multirow{2}{*}{$\begin{array}{c}\text { GRE } \\
\text { Behavior }\end{array}$} & \multirow{2}{*}{$\begin{array}{l}\text { Number of } \\
\text { Events }\end{array}$} & \multirow{2}{*}{\multicolumn{3}{|c|}{$\begin{array}{c}\text { Number of } \\
\text { Deflagrations }\end{array}$}} & \multirow{2}{*}{$\begin{array}{l}\text { Number of } \\
\text { Detonations }\end{array}$} & \multicolumn{5}{|c|}{ \%LFL } & \multirow{2}{*}{\multicolumn{2}{|c|}{$\begin{array}{c}\text { Mean } \\
\text { Number of } \\
\text { Events/year }\end{array}$}} \\
\hline & & & & & & & \multicolumn{3}{|c|}{$\begin{array}{c}\text { Mean } \\
\text { Frequency }\end{array}$} & \multicolumn{2}{|c|}{$\begin{array}{c}\text { Mean } \\
\text { Concentration }\end{array}$} & & \\
\hline \multicolumn{14}{|c|}{ IGNITION CONTROL SET IN/EX-TANK } \\
\hline A-102 & all sizes & 11962 & $=$ & 272 & $<$ & 0 & $=$ & $9.28 \mathrm{E}-03$ & $>$ & 18.70 & $<$ & $7.91 \mathrm{E}-02$ & \\
\hline \multicolumn{14}{|c|}{ VENTILATION FLOW RATE REDUCTION } \\
\hline \multirow[t]{4}{*}{$\mathrm{B}-11 !$} & all sizes & 11828 & $=$ & 116 & $=$ & 0 & $=$ & $6.44 \mathrm{E}-02$ & $=$ & 10.80 & $=$ & $5.93 \mathrm{E}-01$ & $=$ \\
\hline & small & 3940 & $=$ & 0 & $=$ & $\overline{0}$ & $=$ & $1.62 \mathrm{E}-01$ & $=$ & 0.14 & $>$ & $5.22 \mathrm{E}-01$ & \\
\hline & medium & 3944 & $=$ & $\overline{116}$ & $=$ & 0 & $=$ & $2.44 \mathrm{E}-02$ & $=$ & 29.20 & $=1$ & $5.44 \mathrm{E}-02$ & $=$ \\
\hline & large & 3944 & $=$ & 0 & $=$ & 0 & $=$ & $7.08 \mathrm{E}-03$ & $=$ & 3.14 & $>$ & $1.50 \mathrm{E}-02$ & $=$ \\
\hline \multicolumn{14}{|c|}{ VENTILATION FLOW RATE INCREASE } \\
\hline \multirow[t]{4}{*}{$\mathrm{B}-111$} & all sizes & 11828 & $=$ & \begin{tabular}{|l|}
116 \\
\end{tabular} & $\Rightarrow$ & 0 & $=1$ & $6.44 \mathrm{E}-02$ & $\Rightarrow$ & 10.70 & $<$ & $5.93 \mathrm{E}-01$ & $=$ \\
\hline & small & 3940 & $=$ & 0 & $=$ & 0 & $=$ & $1.62 \mathrm{E}-0 \mathrm{I}$ & $=$ & 0.10 & $<$ & $5.22 \mathrm{E}-01$ & $=$ \\
\hline & medium & 3944 & 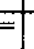 & 116 & $=$ & 0 & $=$ & $2.44 \mathrm{E}-02$ & $=$ & 29.10 & $<$ & $5.44 \mathrm{E}-02$ & $=$ \\
\hline & large & 3944 & $=$ & 0 & $\Rightarrow$ & 0 & $=1$ & $7.08 \mathrm{E}-03$ & $=$ & $2 . \overline{75}$ & $<$ & $1.50 \mathrm{E}-02$ & $=$ \\
\hline \multicolumn{14}{|c|}{ INERTING NITROGEN $5 \%$ OXYGEN } \\
\hline $\mathrm{T}-203$ & all sizes & 11628 & $=1$ & \begin{tabular}{l|}
0 \\
\end{tabular} & $<$ & 0 & $=$ & $8.95 \mathrm{E}-03$ & $=$ & 6.17 & $<$ & $7.7 I \mathrm{E}-02$ & $x$ \\
\hline \multicolumn{14}{|c|}{ INERTING NITROGEN $10 \%$ OXYGEN } \\
\hline $\mathrm{T}-203$ & all sizes & 11628 & $=$ & $\begin{array}{l} \\
\end{array}$ & $<$ & 0 & $=$ & $8.95 \mathrm{E}-03$ & $=$ & 6.17 & $<$ & $7.71 \mathrm{E}-02$ & $=$ \\
\hline \multicolumn{14}{|c|}{ TANK CRACKING PRESSURE REDUCTION } \\
\hline B-111 & all sizes & 11828 & $=$ & 116 & $=1$ & 0 & $=$ & $6.44 \mathrm{E}-02$ & $=$ & 10.80 & $=1$ & $5.93 \mathrm{E}-01$ & $=$ \\
\hline \multicolumn{14}{|c|}{ TANK CRACKING PRESSURE INCREASE } \\
\hline B-111 & all sizes & 11828 & $=1$ & 116 & $\Rightarrow$ & 0 & $=$ & $6.44 \mathrm{E}-02$ & $=$ & 10.80 & $=$ & $5.93 \mathrm{E}-0 \mathrm{I}$ & $=$ \\
\hline \multicolumn{14}{|c|}{ TANK COLLAPSE PRESSURE REDUCTION } \\
\hline B-111 & all sizes & 11828 & $=$ & 116 & $=$ & 0 & $=$ & $6.44 \mathrm{E}-02$ & $=$ & 10.80 & $=$ & $5.93 \mathrm{E}-01$ & \\
\hline \multicolumn{14}{|c|}{ WASTE INTRUSIVE EQUIPMENT - INCREASE OPERATIONS } \\
\hline \multirow[t]{2}{*}{ A-102 } & all sizes & 11963 & $>$ & 272 & $>$ & 1 & $P$ & $9.63 \mathrm{E}-03$ & $>$ & 18.80 & $>$ & $7.91 \mathrm{E}-02$ & \\
\hline & WIE all sizes & 1 & $>$ & 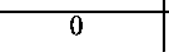 & $=$ & 1 & $>$ & $1.00 \mathrm{E}+00$ & $>$ & 667.00 & $>$ & $1.00 \mathrm{E}+00$ & \\
\hline \multicolumn{14}{|c|}{ WASTE INTRUSIVE EQUIPMENT - INCREASE OPERATIONS - NO PURGE } \\
\hline \multirow[t]{2}{*}{ A-102 } & all sizes & 12670 & $>1$ & 584 & $>1$ & 396 & $>$ & $6.46 \mathrm{E}-02$ & $>$ & 43.80 & $>$ & $7.91 \mathrm{E}-02$ & $=$ \\
\hline & WIE all sizes & 708 & $>$ & 312 & $>$ & 396 & $>$ & $1.00 \mathrm{E}+00$ & $=$ & 467.00 & $<$ & $1.00 \mathrm{E}+00$ & $=$ \\
\hline \multicolumn{14}{|c|}{ WASTE INTRUSIVE EQUIPMENT - INCREASE OPERATIONS - IINCREASE EQUIPMENT DIAMETER } \\
\hline$\overline{A-102}$ & all sizes & 11963 & $=$ & 272 & $=$ & 1 & $=$ & $9.63 \mathrm{E}-03$ & $=$ & 18.80 & $\because$ & $7.91 \mathrm{E}-02$ & $=$ \\
\hline & WIE all sizes & 1 & $=$ & 0 & $=$ & 1 & $=1$ & $1.00 \mathrm{E}+00$ & $=$ & 667.00 & $=$ & $1.00 \mathrm{E}+00$ & $=$ \\
\hline & STE INTRU & & & & & & & UNCREASE & & LIPMENT I & & & \\
\hline$A-102$ & all sizes & 11963 & $=$ & 272 & $=$ & 1 & $=$ & $9.63 \mathrm{E}-03$ & $=$ & 18.80 & $=$ & $7.91 \mathrm{E}-02$ & $=$ \\
\hline & WIE all sizes & 1 & $=$ & 0 & $=$ & I & $=$ & $1.00 \mathrm{E}+00$ & $=$ & 667.00 & $=$ & $1.00 \mathrm{E}+00$ & \\
\hline
\end{tabular}


RPP-6888 REV 0

APPENDIX B

DST ACCEPTANCE TEST RESULTS

B-1 
RPP-6888 REV 0

This page left intentionally blank.

B-2 


\title{
RPP-6888 REV 0
}

\begin{abstract}
APPENDIX B
This Appendix provides the results of the DST acceptance testing. A strict comparison of the benchmark results to the trend analysis results does not consider the magnitude of the difference. For example, if the benchmark mean consequence value is $4.567 \mathrm{E}-05 \mathrm{~Sv}$ and the mean trend test analysis result is $4.566 \mathrm{E}-05 \mathrm{~Sv}$, a comparison of this type would indicate that by modifying a specific parameter, the consequences decreased; however, based on the conservatisms and uncertainties incorporated in the Analysis Tool, a better conclusion would be that there is no change.
\end{abstract}


RPP-6888 REV 0

This page left intentionally blank

B-4 


\section{RPP-6888 REV 0}

Table B-1. DST Acceptance Test Results: GRE Behavior. (Seed: 90,000,000, Sample Count: 1000)

\begin{tabular}{|c|c|c|c|c|c|c|c|c|c|c|c|c|c|}
\hline \multirow[t]{2}{*}{ Tank } & \multirow{2}{*}{$\begin{array}{c}\text { GRE } \\
\text { Behavior }\end{array}$} & \multirow{2}{*}{$\begin{array}{l}\text { Number of } \\
\text { Events }\end{array}$} & \multirow{2}{*}{\multicolumn{3}{|c|}{\begin{tabular}{c|} 
Number of \\
Deflagrations
\end{tabular}}} & \multirow{2}{*}{$\begin{array}{l}\text { Number of } \\
\text { Detonations }\end{array}$} & \multicolumn{5}{|c|}{$\% \mathrm{LFL}$} & \multirow{2}{*}{$\begin{array}{c}\text { Mean } \\
\text { Number of } \\
\text { Events/year }\end{array}$} & \\
\hline & & & & & & & & $\begin{array}{c}\text { Mean } \\
\text { Frequency }\end{array}$ & & $\begin{array}{c}\text { Mean } \\
\text { Concentration }\end{array}$ & & & \\
\hline \multicolumn{14}{|c|}{ BENCHMARK } \\
\hline \multirow[t]{6}{*}{$\overline{A N}-107$} & all sizes & 4235 & & 505 & & 0 & & $7.07 \mathrm{E}+00$ & & $1.26 \mathrm{E}+02$ & & $3.33 \mathrm{E}+01$ & \\
\hline & small & 1221 & & 0 & & 0 & & $5.31 \mathrm{E}+00$ & & $1.81 \mathrm{E}+00$ & & N/A & \\
\hline & medium & 2014 & & 492 & & 0 & & $1.16 \mathrm{E}+01$ & & $2.47 \mathrm{E}+02$ & & $\mathrm{~N} / \mathrm{A}$ & \\
\hline & large & 1000 & & 13 & & 0 & & $2.4 \overline{\mathrm{E}}-03$ & & $3.54 \mathrm{E}+01$ & & $2.43 \mathrm{E}-03$ & \\
\hline & BD & 1241 & & 0 & & 0 & & $2.41 \mathrm{E}+0 \mathrm{I}$ & & $6.79 \mathrm{E}+00$ & & $3.33 \mathrm{E}+01$ & \\
\hline & Non-BD & 2994 & & 505 & & 0 & & $2.91 \mathrm{E}-03$ & & $1.76 E+02$ & & $8.73 \mathrm{E}-03$ & \\
\hline \multirow[t]{6}{*}{$\mathrm{AW}-106$} & all sizes & 12017 & & 936 & & 0 & & $1.95 \mathrm{E}-01$ & & $6.08 \mathrm{E}+01$ & & $1.28 \mathrm{E}+00$ & \\
\hline & small & 4001 & & 0 & & 0 & & $4.42 \mathrm{E}-01$ & & $5.83 \mathrm{E}-01$ & & $9.86 \mathrm{E}-0 \mathrm{I}$ & \\
\hline & medium & 4008 & & 930 & & 0 & & $1.18 \mathrm{E}-0 \mathrm{I}$ & & $1.65 \overline{\mathrm{E}}+02$ & & $2.39 \mathrm{E}-01$ & \\
\hline & large & 4008 & & 6 & & 0 & & $2.42 \mathrm{E}-02$ & & $1.71 \mathrm{E}+01$ & & $4,90 \mathrm{E}-02$ & \\
\hline & $\mathrm{BD}$ & 0 & & 0 & & 0 & & $0.00 \mathrm{E}+00$ & & $0.00 \mathrm{E}+00$ & & $0.00 \mathrm{E}+00$ & \\
\hline & Non-BD & 12017 & & 936 & & 0 & & $1.95 \mathrm{E}-01$ & & $6.08 \mathrm{E}+01$ & & $1.27 \mathrm{E}+00$ & \\
\hline \multirow[t]{6}{*}{$\mathrm{AY}-\overline{101}$} & all sizes & 11997 & & 80 & & 0 & & $8.03 \mathrm{E}-01$ & & $7.66 \mathrm{E}+00$ & & $5 . \overline{56 \mathrm{E}+00}$ & \\
\hline & sinall & 3997 & & 0 & & 0 & & $1.52 \mathrm{E}+00$ & & $2.61 \mathrm{E}-01$ & & $3.76 \mathrm{E}+00$ & \\
\hline & medium & 4000 & & 80 & & 0 & & $4.75 \mathrm{E}-0 \mathrm{I}$ & & $2.18 \mathrm{E}+01$ & & $9.73 \mathrm{E}-01$ & \\
\hline & large & 4000 & & 0 & & 0 & & $4.11 \mathrm{E}-01$ & & $9.33 \mathrm{E}-01$ & & $8.24 \mathrm{E}-01$ & \\
\hline & $\mathrm{BD}$ & 0 & & 0 & & 0 & & $0.00 \mathrm{E}+00$ & & $0.00 \mathrm{E}+00$ & & $0.00 \mathrm{E}+00$ & \\
\hline & Non-BD & 11997 & & 80 & & 0 & & $8.03 \mathrm{E}-0 \mathrm{I}$ & & $7.66 \mathrm{E}+00$ & & $5.56 \mathrm{E}+00$ & \\
\hline \multicolumn{14}{|c|}{ INERTING NITROGEN $5 \%$ OXYGEN } \\
\hline AN-107 & all sizes & 4235 & $=1$ & 133 & $<$ & 0 & $=$ & $7.07 \mathrm{E}+00$ & $=$ & $5.48 \mathrm{E}+01$ & $<$ & $3 . \overline{33 E+01}$ & $=$ \\
\hline \multicolumn{14}{|c|}{ INERTING NITROGEN $10 \%$ OXYGEN } \\
\hline$A N-107$ & all sizes & 4235 & $=$ & 133 & $<$ & 0 & $=$ & $7.07 \mathrm{E}+00$ & $=$ & $5.48 \mathrm{E}+01$ & $<$ & $3.33 \mathrm{E}+01$ & $=$ \\
\hline \multicolumn{14}{|c|}{ WASTE VOLUME REDUCTION } \\
\hline \multirow[t]{3}{*}{ AN-107 } & all sizes & 4355 & $>$ & 463 & $<$ & 0 & $=$ & $8.88 \mathrm{E}+00$ & $>$ & $1.01 \mathrm{E}+02$ & $<$ & $1.01 \mathrm{E}+02$ & $>$ \\
\hline & $\mathrm{BD}$ & 1361 & $>$ & 0 & $=$ & 0 & $=$ & $2.84 \overline{\mathrm{E}}+01$ & $>$ & $3.66 \mathrm{E}+00$ & $<$ & $3.66 \mathrm{E}+00$ & $<$ \\
\hline & Non-BD & 2994 & $=$ & 463 & $<$ & 0 & $=$ & $2.91 \mathrm{E}-03$ & $=$ & $1 . \overline{46} \overline{\mathrm{E}+02}$ & $<$ & $1.46 \mathrm{E}+02$ & $>$ \\
\hline \multicolumn{14}{|c|}{ VENTILATION FLOW RATE REDUCTION } \\
\hline \multirow[t]{6}{*}{ AW-106 } & all sizes & 12017 & $=1$ & 940 & $>$ & 0 & $=$ & $1.95 \mathrm{E}-01$ & $=$ & $6.26 \mathrm{E}+01$ & $>$ & $1.28 \mathrm{E}+00$ & $=$ \\
\hline & small & 4001 & $=$ & 0 & $=$ & 0 & $=$ & $4.42 \mathrm{E}-01$ & $=$ & $8.74 \mathrm{E}-01$ & $>$ & $9.86 \mathrm{E}-01$ & $=$ \\
\hline & medium & 4008 & $=$ & $\overline{932}$ & $>$ & 0 & $=$ & $1.18 \mathrm{E}-01$ & $=$ & $1.65 \mathrm{E}+02$ & $=$ & $2 . \overline{39 \mathrm{E}-01}$ & $=$ \\
\hline & large & 4008 & $=$ & 8 & $>$ & 0 & $=$ & $2.42 \mathrm{E}-02$ & $=$ & $2.16 \mathrm{E}+01$ & $>$ & $4.90 \mathrm{E}-02$ & $=$ \\
\hline & $\overline{B D}$ & 0 & $=$ & 0 & $=$ & 0 & $=$ & $0.00 \overline{\mathrm{E}}+00$ & $=$ & $0.00 \mathrm{E}+00$ & $=$ & $0.00 \mathrm{E}+00$ & $=$ \\
\hline & Non-BD & 12017 & $=$ & 940 & $>$ & 0 & $=$ & $1.95 \mathrm{E}-01$ & $=$ & $6.26 \mathrm{E}+01$ & $>$ & $1.27 \mathrm{E}+00$ & $=$ \\
\hline \multicolumn{14}{|c|}{ VENTILATION FLOW RATE INCREASE } \\
\hline $\mathrm{AW}-106$ & all sizes & 12017 & $=$ & 932 & $<$ & 0 & $=$ & $1.95 \mathrm{E}-01$ & $=$ & $5.85 \mathrm{E}+01$ & $<$ & $1.28 \mathrm{E}+00$ & $=$ \\
\hline & small & $400 !$ & $=$ & 0 & $=$ & 0 & $=$ & $4.42 \mathrm{E}-01$ & $=$ & $3.80 \mathrm{E}-0 \mathrm{I}$ & $<$ & $9 . \overline{86 E-01}$ & $=$ \\
\hline & medium & 4008 & $=$ & 930 & $=$ & 0 & $=$ & $1.18 \mathrm{E}-01$ & $=$ & $1.64 \mathrm{E}+02$ & $<$ & $2.39 \mathrm{E}-01$ & $=$ \\
\hline & large & 4008 & $=$ & 2 & $<$ & 0 & $=$ & $2.42 \mathrm{E}-02$ & $=$ & $1.13 \mathrm{E}+0 \mid$ & $<$ & $4.90 \mathrm{E}-02$ & $=$ \\
\hline & $\mathrm{BD}$ & 0 & $=$ & 0 & $=$ & 0 & $=$ & $0.00 \mathrm{E}+00$ & $=$ & $0.00 \mathrm{E}+00$ & $=$ & $0.00 \mathrm{E}+00$ & $=$ \\
\hline & Non-BD & 12017 & $=$ & 932 & $<$ & 0 & $=$ & $1.95 \mathrm{E}-01$ & $=$ & $5.85 \mathrm{E}+01$ & $<$ & $1.27 \mathrm{E}+00$ & $=$ \\
\hline & & & & RUSIVE OPE & $R A$ & ITIONS INCR & EA & SE ALL & & & & & \\
\hline AW-106 & all sizes & 12017 & $=$ & 936 & $=$ & 0 & $=$ & $1.95 \mathrm{E}-0 \mathrm{l}$ & $=$ & $6.08 \mathrm{E}+01$ & $=$ & $1.28 \mathrm{E}+00$ & $=$ \\
\hline & small & 4001 & $=$ & 0 & $=$ & 0 & $=$ & $4.42 \mathrm{E}-01$ & $=$ & $5.83 \mathrm{E}-01$ & $=$ & $9.86 \mathrm{E}-01$ & $=$ \\
\hline
\end{tabular}




\section{RPP-6888 REV 0}

Table B-1. DST Acceptance Test Results: GRE Behavior. (Seed: 90,000,000, Sample Count: 1000)

\begin{tabular}{|c|c|c|c|c|c|c|c|c|c|c|c|c|c|}
\hline \multirow[t]{2}{*}{ Tank } & \multirow{2}{*}{$\begin{array}{c}\text { GRE } \\
\text { Behavior }\end{array}$} & \multirow{2}{*}{$\begin{array}{l}\text { Number of } \\
\text { Events }\end{array}$} & \multirow{2}{*}{\multicolumn{2}{|c|}{$\begin{array}{c}\text { Number of } \\
\text { Deflagrations }\end{array}$}} & \multirow[b]{3}{*}{$=$} & \multirow{2}{*}{$\begin{array}{l}\text { Number of } \\
\text { Detonations }\end{array}$} & \multicolumn{5}{|c|}{$\%$ LFL } & \multirow{2}{*}{$\begin{array}{c}\text { Mean } \\
\text { Number of } \\
\text { Events/year }\end{array}$} & \\
\hline & & & & & & & \multicolumn{3}{|c|}{$\begin{array}{c}\text { Mean } \\
\text { Frequency }\end{array}$} & \multicolumn{2}{|c|}{$\begin{array}{c}\text { Mean } \\
\text { Concentration }\end{array}$} & & \\
\hline & medium & 4008 & $=$ & 930 & & 0 & $=$ & $1.18 \mathrm{E}-01$ & $=$ & $1.65 E+02$ & $=$ & $2.39 \mathrm{E}-0 \mathrm{I}$ & $=$ \\
\hline & Targe & 4008 & $=$ & 6 & $=$ & 0 & $=$ & $2.42 \mathrm{E}-02$ & $=$ & $1.71 \mathrm{E}+01$ & $=$ & $4.90 \mathrm{E}-02$ & $=$ \\
\hline & $\mathrm{BD}$ & 0 & $=$ & 0 & $=$ & 0 & $=$ & $0.00 \mathrm{E}+00$ & $=$ & $0.00 \mathrm{E}+00$ & $=1$ & $0.00 \mathrm{E}+00$ & $=$ \\
\hline & Non-BD & 12017 & $=$ & 936 & $=$ & 0 & $=$ & $1.95 \mathrm{E}-01$ & $=$ & $6.08 \mathrm{E}+01$ & $=$ & $1.27 \mathrm{E}+00$ & $=$ \\
\hline \multicolumn{14}{|c|}{ INTRUSIVE OPERATIONS INCREASE NON WASTE DISTURBING } \\
\hline \multirow[t]{6}{*}{$\mathrm{AW}-306$} & all sizes & 12017 & $=$ & 936 & $=$ & 0 & $=1$ & $1.95 \mathrm{E}-01$ & $=$ & $6.08 \mathrm{E}+01$ & $=$ & $1.28 \mathrm{E}+00$ & $=$ \\
\hline & small & 4001 & $=$ & 0 & $=$ & 0 & $=1$ & $4.42 \mathrm{E}-01$ & $=$ & $5.83 \mathrm{E}-01$ & $=$ & $9.86 \mathrm{E}-01$ & $=$ \\
\hline & medium & 4008 & $=$ & 930 & $=$ & 0 & $=$ & $1.18 \mathrm{E}-0 \mathrm{I}$ & $=$ & $1.65 \mathrm{E}+02$ & $=$ & $2.39 \mathrm{E}-01$ & $=$ \\
\hline & large & 4008 & $=$ & 6 & $=$ & 0 & $=$ & $2.42 \mathrm{E}-02$ & $=$ & $1.71 \mathrm{E}+01$ & $=$ & $4.90 \mathrm{E}-02$ & $=$ \\
\hline & $\mathrm{BD}$ & 0 & $=$ & 0 & $=$ & 0 & $=$ & $\overline{0.00 \mathrm{E}}+00$ & $=1$ & $0.00 \mathrm{E}+00$ & $=$ & $0.00 \mathrm{E}+00$ & $=$ \\
\hline & Non-BD & 12017 & $=$ & 936 & $=$ & 0 & $=$ & $1.95 \mathrm{E}-01$ & $=$ & $6.08 \mathrm{E}+01$ & $=$ & $1.27 \mathrm{E}+00$ & $=$ \\
\hline \multicolumn{14}{|c|}{ INTRUSIVE OPERATIONS INCREASE LOCALLY WASTE DISTURBING } \\
\hline \multirow[t]{6}{*}{ AW-106 } & all sizes & 12147 & $>$ & 954 & $>$ & 0 & $=$ & $1.85 \mathrm{E}-01$ & $<$ & $6.18 \mathrm{E}+01$ & $>$ & $1.22 \mathrm{E}+00$ & $<$ \\
\hline & small & 4043 & $>$ & 0 & $=1$ & 0 & $=$ & $4.10 \mathrm{E}-01$ & $<$ & $5.84 \mathrm{E}-0 \mathrm{I}$ & $>$ & $9.24 \mathrm{E}-01$ & $<$ \\
\hline & medium & 4052 & $>$ & 952 & $>$ & 0 & $=$ & $1.22 \mathrm{E}-01$ & $>$ & $1.68 \mathrm{E}+02$ & $>$ & $2.46 \mathrm{E}-01$ & $>$ \\
\hline & large & 4052 & $>$ & 2 & $<$ & 0 & $=$ & $2.53 \mathrm{E}-02$ & $>$ & $1.71 \mathrm{E}+01$ & $=$ & $5.12 \mathrm{E}-02$ & $>$ \\
\hline & $\overline{\mathrm{BD}}$ & 0 & $=$ & 0 & $=$ & 0 & $=$ & $0.00 \mathrm{E}+00$ & $=$ & $0.00 \mathrm{E}+00$ & $=$ & $0.00 \mathrm{E}+00$ & $=$ \\
\hline & Non-BD & 12147 & $>$ & 954 & $>$ & 0 & $=$ & $1.85 \mathrm{E}-01$ & $<$ & $6.18 \mathrm{E}+01$ & $>$ & $1.22 \mathrm{E}+00$ & $<$ \\
\hline \multicolumn{14}{|c|}{ INTRUSIVE OPERATIONS INCREASE GLOBALLY WASTE DISTURBING } \\
\hline \multirow[t]{6}{*}{ AW-106 } & all sizes & 12125 & $>$ & 936 & $=1$ & 0 & $=1$ & $2.09 \mathrm{E}-01$ & $>$ & $6.09 \mathrm{E}+0 \mathrm{I}$ & $>$ & $1.36 \mathrm{E}+00$ & $>$ \\
\hline & small & 4037 & $>$ & 0 & $=$ & 0 & $=$ & $4.82 \mathrm{E}-01$ & $>$ & $5.80 \mathrm{E}-0 \mathrm{I}$ & $<$ & $1.06 \mathrm{E}+00$ & $>$ \\
\hline & medium & 4044 & $>$ & 930 & $\Rightarrow$ & 0 & $=$ & $1.22 \mathrm{E}-01$ & $>$ & $1.65 \mathrm{E}+02$ & $=$ & $2.46 \mathrm{E}-01$ & $>$ \\
\hline & large & 4044 & $>$ & 6 & $=$ & 0 & $=$ & $2.41 \mathrm{E}-02$ & $<$ & $1.71 \mathrm{E}+01$ & $=$ & $4.88 \mathrm{E}-02$ & $<$ \\
\hline & $\mathrm{BD}$ & 0 & $=$ & 0 & $=$ & 0 & $=$ & $0.00 \mathrm{E}+00$ & $=$ & $0.00 \mathrm{E}+00$ & $=$ & $0.00 \mathrm{E}+00$ & $=$ \\
\hline & Non-BD & 12125 & $>$ & 936 & $=$ & 0 & $=$ & $2.09 \mathrm{E}-01$ & $>$ & $6.09 \mathrm{E}+01$ & $>$ & $1.35 \mathrm{E}+00$ & $>$ \\
\hline \multicolumn{14}{|c|}{ INTRUSIVE OPERATIONS INCREASE EX-TANK } \\
\hline \multirow[t]{6}{*}{$\mathrm{AW}-106$} & all sizes & 12039 & $>$ & 936 & $=$ & 0 & $=$ & $1.83 \mathrm{E}-01$ & $<$ & $5.78 \mathrm{E}+01$ & $<1$ & $1.23 \mathrm{E}+00$ & $<$ \\
\hline & small & 4007 & $>$ & 0 & $=$ & 0 & $=$ & $4.19 \mathrm{E}-01$ & $<$ & $5.92 \bar{E}-01$ & $>$ & $9.62 \mathrm{E}-01$ & $<$ \\
\hline & medium & 4016 & $>$ & 936 & $>$ & 0 & $=$ & $1.08 \mathrm{E}-01$ & $<$ & $1.55 \mathrm{E}+02$ & $<$ & $2.19 \mathrm{E}-01$ & $<$ \\
\hline & large & 4016 & $>$ & 0 & $<$ & 0 & $=$ & $2.33 \mathrm{E}-02$ & $<$ & $1.72 \mathrm{E}+0 \mathrm{I}$ & $>$ & $4.74 \mathrm{E}-02$ & $<$ \\
\hline & $\mathrm{BD}$ & 0 & $=$ & 0 & $=$ & 0 & $=$ & $0.00 \mathrm{E}+00$ & $=$ & $0.00 \mathrm{E}+00$ & $=$ & $0.00 \mathrm{E}+00$ & $=$ \\
\hline & Non-BD & 12039 & $>$ & 936 & $=$ & $\overline{0}$ & $=$ & $1.83 \mathrm{E}-0 \mathrm{I}$ & $<\mid$ & $5.78 \mathrm{E}+01$ & $<$ & $1.23 \mathrm{E}+00$ & $<$ \\
\hline \multicolumn{14}{|c|}{ INTRUSIVE OPERATIONS INCREASE IN/EX-TANK } \\
\hline$A W-106$ & all sizes & 12063 & $>$ & 918 & $<$ & 0 & $=$ & $2.01 \mathrm{E}-01$ & $>$ & $5.97 \mathrm{E}+01$ & $<$ & $1.29 \mathrm{E}+00$ & $>$ \\
\hline & small & 4015 & $>$ & 0 & $=$ & 0 & $=$ & $4.51 \mathrm{E}-0 \mathrm{I}$ & $>$ & $5.88 \mathrm{E}-01$ & $>1$ & $9.81 \mathrm{E}-01$ & $<$ \\
\hline & medium & 4024 & $>$ & 914 & $<$ & 0 & $=$ & $1.26 \mathrm{E}-01$ & $>$ & $1.61 \mathrm{E}+02$ & $<$ & $2.53 \mathrm{E}-01$ & $>$ \\
\hline & large & 4024 & $>$ & 4 & $<$ & 0 & $=$ & $2.51 \mathrm{E}-02$ & $>$ & $1.71 E+01$ & $=$ & $5.07 \mathrm{E}-02$ & $>$ \\
\hline & $\mathrm{BD}$ & 0 & $=$ & 0 & $=$ & 0 & $=$ & $0.00 \mathrm{E}+00$ & $=$ & $0.00 \mathrm{E}+00$ & $=$ & $0.00 \mathrm{E}+00$ & $=$ \\
\hline & Non-BD & 12063 & $>$ & 918 & $<$ & 0 & $=$ & $2.01 \mathrm{E}-01$ & $>$ & $5 . \overline{97 \mathrm{E}+01}$ & $<$ & $1.29 \mathrm{E}+00$ & $>$ \\
\hline & & & & IGNITION CC & $\mathbf{D N}$ & TROL SET IN & 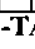 & NK & & & & & \\
\hline $\mathrm{AY}-101$ & all sizes & 11997 & $=$ & 80 & $\Rightarrow$ & 0 & $=$ & 8.03E-01 & $=1$ & $7.66 \mathrm{E}+00$ & $=$ & $5.56 \mathrm{E}+00$ & $=$ \\
\hline & & & & IGNITION CO & $\bar{N}$ & TROL SET EX & $-T$ & NK & & & & & \\
\hline $\mathrm{AY}-101$ & all sizes & 11997 & $=$ & 80 & $=$ & 0 & $=$ & $8.03 \mathrm{E}-01$ & $=1$ & $7.66 \mathrm{E}+00$ & $=$ & $5.56 \mathrm{E}+00$ & $=$ \\
\hline & & & & GNITION CON & si & ROL SET IN/H & $\lambda-$ & ANK & & & & & \\
\hline$A Y-101$ & all sizes & 11997 & $=$ & 80 & $=1$ & 0 & $=$ & $8.03 \bar{E}-01$ & $=$ & $7.66 \mathrm{E}+00$ & $=$ & $5.56 \mathrm{E}+00$ & $=$ \\
\hline
\end{tabular}




\section{RPP-6888 REV 0}

Table B-1. DST Acceptance Test Results: GRE Behavior. (Seed: 90,000,000, Sample Count: 1000)

\begin{tabular}{|c|c|c|c|c|c|c|c|c|c|c|c|c|c|}
\hline \multirow[t]{2}{*}{ Tank } & \multirow{2}{*}{$\begin{array}{c}\text { GRE } \\
\text { Behavior }\end{array}$} & \multirow{2}{*}{$\begin{array}{l}\text { Number of } \\
\text { Events }\end{array}$} & \multirow{2}{*}{\multicolumn{2}{|c|}{$\begin{array}{c}\text { Number of } \\
\text { Deflagrations }\end{array}$}} & \multirow[b]{3}{*}{$>$} & \multirow{2}{*}{$\begin{array}{l}\text { Number of } \\
\text { Detonations }\end{array}$} & \multicolumn{5}{|c|}{$\% \mathbf{L F L}$} & \multirow{2}{*}{$\begin{array}{c}\text { Mean } \\
\text { Number of } \\
\text { Events/year }\end{array}$} & \\
\hline & & & & & & & \multicolumn{3}{|c|}{$\begin{array}{c}\text { Mean } \\
\text { Frequency }\end{array}$} & \multicolumn{2}{|c|}{$\begin{array}{c}\text { Mean } \\
\text { Concentration }\end{array}$} & & \\
\hline \multirow[t]{3}{*}{ AN-107 } & all sizes & 4230 & $<$ & 1781 & & 45 & $>$ & $8.94 \mathrm{E}-01$ & $<$ & $2.42 \mathrm{E}+02$ & $P$ & $5.94 \mathrm{E}+00$ & $<$ \\
\hline & BD & 1253 & $>$ & 124 & $>$ & 0 & $=1$ & $3.01 \mathrm{E}+00$ & $<$ & $5.67 \mathrm{E}+01$ & $>$ & $5.92 \mathrm{E}+00$ & $<$ \\
\hline & Non-BD & 2977 & $<$ & 1657 & $>$ & $\overline{45}$ & $>$ & $2.92 \mathrm{E}-03$ & $>$ & $3.21 \mathrm{E}+02$ & $>$ & $8.77 \mathrm{E}-03$ & $>$ \\
\hline \multicolumn{14}{|c|}{ DENSITY RATIO LIQUIDS TO IMMOBILE SOLIDS } \\
\hline \multirow[t]{3}{*}{ AN-107 } & all sizes & 4235 & $=$ & 505 & $=$ & 0 & $=1$ & $7.07 \mathrm{E}+00$ & $=1$ & $1.26 \mathrm{E}+02$ & $=$ & $3.33 \mathrm{E}+01$ & $=$ \\
\hline & $\mathrm{BD}$ & 1241 & $=$ & 0 & $=$ & 0 & $=$ & $\overline{2.41 \mathrm{E}} \overline{+01}$ & $=$ & $6.79 \mathrm{E}+00$ & $=$ & $3.33 \mathrm{E}+01$ & $=$ \\
\hline & Non-BD & 2994 & $=$ & 505 & $=$ & 0 & $=$ & $2.91 \mathrm{E}-03$ & $=$ & $1.76 \mathrm{E}+02$ & $=$ & $8.73 \mathrm{E}-03$ & $=$ \\
\hline \multicolumn{14}{|c|}{ GAS GENERATION RATE INCREASE SETTLED SOLIDS } \\
\hline \multirow[t]{6}{*}{ AN-107 } & all sizes & 4235 & $=$ & \begin{tabular}{|l|l|}
505 &
\end{tabular} & $=$ & 0 & $=$ & $7.06 \mathrm{E}+0 \mathrm{I}$ & $>$ & $1.26 \mathrm{E}+02$ & $=$ & $3.02 \mathrm{E}+02$ & 7 \\
\hline & small & 1221 & $=$ & 0 & $=$ & 0 & $=$ & $5.23 \mathrm{E}+01$ & $>$ & $1.81 \mathrm{E}+00$ & $=$ & $\mathrm{N} / \mathrm{A}$ & $=$ \\
\hline & medium & 2014 & $=$ & 492 & $=$ & 0 & $=$ & $1.17 \overline{\mathrm{E}}+02$ & $>$ & $2.47 \mathrm{E}+02$ & $=$ & N/A & $=$ \\
\hline & large & 1000 & $=$ & 13 & $=$ & 0 & $=$ & $2.43 \mathrm{E}-03$ & $=$ & $3.54 \mathrm{E}+01$ & $=$ & $2.43 \mathrm{E}-03$ & $=$ \\
\hline & $\overline{\mathrm{BD}}$ & 1241 & $=$ & 0 & $=$ & 0 & $=$ & $2.41 \mathrm{E}+02$ & $>$ & $6.79 E+00$ & $=$ & $3.02 \mathrm{E}+02$ & $>$ \\
\hline & Non-BD & 2994 & $=$ & 505 & $=$ & 0 & $=$ & $2.91 \mathrm{E}-03$ & $=$ & $1.76 \mathrm{E}+02$ & $=$ & $8.73 E-03$ & $=$ \\
\hline \multicolumn{14}{|c|}{ GAS GENERATION RATE INCREASE IMMOBILE SOLIDS } \\
\hline \multirow[t]{6}{*}{ AN-107 } & all sizes & 4235 & $=$ & 505 & $=1$ & 0 & $\Rightarrow$ & $2.25 \mathrm{E}-05$ & $<$ & $4.11 \mathrm{E}-04$ & $<$ & $3.65 \mathrm{E}-04$ & $<$ \\
\hline & small & 1221 & $=$ & 0 & $=$ & 0 & $=$ & $2.92 \mathrm{E}-05$ & $<$ & $7.48 \mathrm{E}-04$ & $<$ & $2.92 \mathrm{E}-05$ & $<$ \\
\hline & medium & 2014 & $=$ & 492 & $=$ & 0 & $=$ & $0.00 \mathrm{E}+00$ & $<$ & $0.00 \mathrm{E}+00$ & $<$ & $0.00 \mathrm{E}+00$ & $<$ \\
\hline & large & 1000 & $=$ & 13 & $=$ & 0 & $\Rightarrow$ & $3.37 \mathrm{E}-05$ & $<$ & $1.77 \mathrm{E}+01$ & $<$ & $1.50 \mathrm{E}-03$ & $<$ \\
\hline & $\mathrm{BD}$ & 1241 & $=$ & 0 & $=$ & 0 & $=$ & $4.77 \mathrm{E}-05$ & $<$ & $7.27 \mathrm{E}-04$ & $<$ & $5.80 \mathrm{E}-04$ & $<$ \\
\hline & Non-BD & 2994 & $=$ & 505 & $=$ & 0 & $=$ & $2.25 \mathrm{E}-05$ & $<$ & $4.63 \mathrm{E}+00$ & $<$ & $3.65 \mathrm{E}-04$ & $<$ \\
\hline \multicolumn{14}{|c|}{ TANK FAILURE PRESSURE REDUCTION } \\
\hline AN-107 & all sizes & 4235 & $=$ & 505 & $=$ & 505 & $>1$ & $0.00 \overline{\mathrm{E}}+00$ & $<$ & $1.26 \mathrm{E}+02$ & $=$ & $3 . \overline{33 \mathrm{E}+01}$ & $=$ \\
\hline \multicolumn{14}{|c|}{ WASTE INTRUSIVE EQUIPMENT - INCREASE OPERATIONS } \\
\hline \multirow[t]{2}{*}{ AY-101 } & all sizes & 11998 & $>$ & 80 & $=$ & 1 & $>$ & $8.03 \mathrm{E}-01$ & $=$ & $7.79 \mathrm{E}+00$ & $>$ & $5.56 \mathrm{E}+00$ & $=$ \\
\hline & WIE all sizes & 1 & $>$ & \begin{tabular}{|l|}
0 \\
\end{tabular} & $>$ & 1 & $>$ & $1.00 \mathrm{E}+00$ & $>$ & $1.55 \mathrm{E}+03$ & $>$ & $1.00 \mathrm{E}+00$ & $>$ \\
\hline \multicolumn{14}{|c|}{ WASTE INTRUSIVE EQUIPMENT - INCREASE OPERATIONS - NO PURGE } \\
\hline \multirow[t]{2}{*}{ AY-101 } & all sizes & 12678 & $>$ & 380 & $>$ & 381 & $>$ & $8.14 \mathrm{E}-01$ & $>$ & $3.29 \mathrm{E}+01$ & $>$ & $5.56 \mathrm{E}+00$ & $=$ \\
\hline & WIE all sizes & 681 & $>$ & 300 & $>$ & 381 & $>$ & $1.00 \overline{\mathrm{E}}+00$ & $=$ & $4.78 \mathrm{E}+02$ & $<$ & $1.00 \mathrm{E}+00$ & $=$ \\
\hline \multicolumn{14}{|c|}{ WASTE INTRUSIVE EQUIPMENT - JNCREASE OPERATIONS - INCREASE EQUIPMENT DIAMETER } \\
\hline \multirow[t]{2}{*}{ AY-101 } & all sizes & 11998 & $=$ & 80 & $=$ & 80 & $>$ & $1.00 \mathrm{E}+00$ & $>$ & $7.79 \mathrm{E}+00$ & $=$ & $5.56 \mathrm{E}+00$ & $=$ \\
\hline & WIE all sizes & 1 & $=$ & 0 & $=$ & 0 & $<$ & $1.00 \mathrm{E}+00$ & $=$ & $1.55 \mathrm{E}+03$ & $=$ & $1.00 \mathrm{E}+00$ & $=$ \\
\hline & STE INTR & IVE EQI & & TT-INCRE & SE: & OPERATION & & NCREASE & $\overrightarrow{Q 1}$ & IIPMENT LF & & & \\
\hline AY-101 & all sizes & 11998 & $F$ & 80 & $=$ & 80 & $>$ & $1.00 \mathrm{E}+00$ & $>$ & $7.79 \mathrm{E}+00$ & $=$ & $5.56 \mathrm{E}+00$ & $=$ \\
\hline & WIE all sizes & 1 & $=$ & 0 & $=$ & 0 & $<$ & $1.00 \mathrm{E}+00$ & $=$ & $1.55 \mathrm{E}+03$ & $=$ & $1.00 \mathrm{E}+00$ & $=$ \\
\hline
\end{tabular}


RPP-6888 REV 0

This page intentionally left blank

B-8 
RPP-6888, Rev. 0

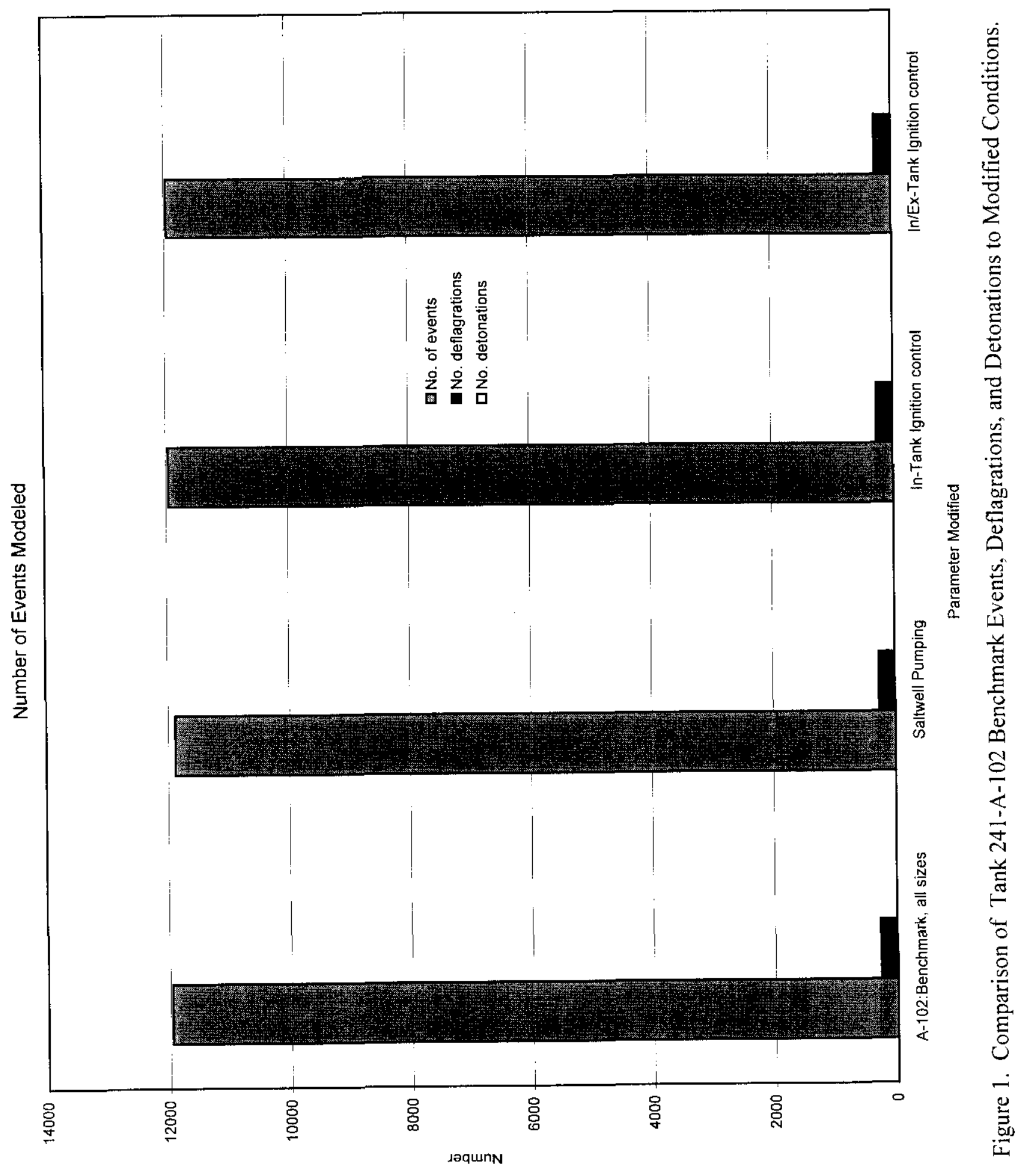

F-1 
RPP-6888, Rev. 0

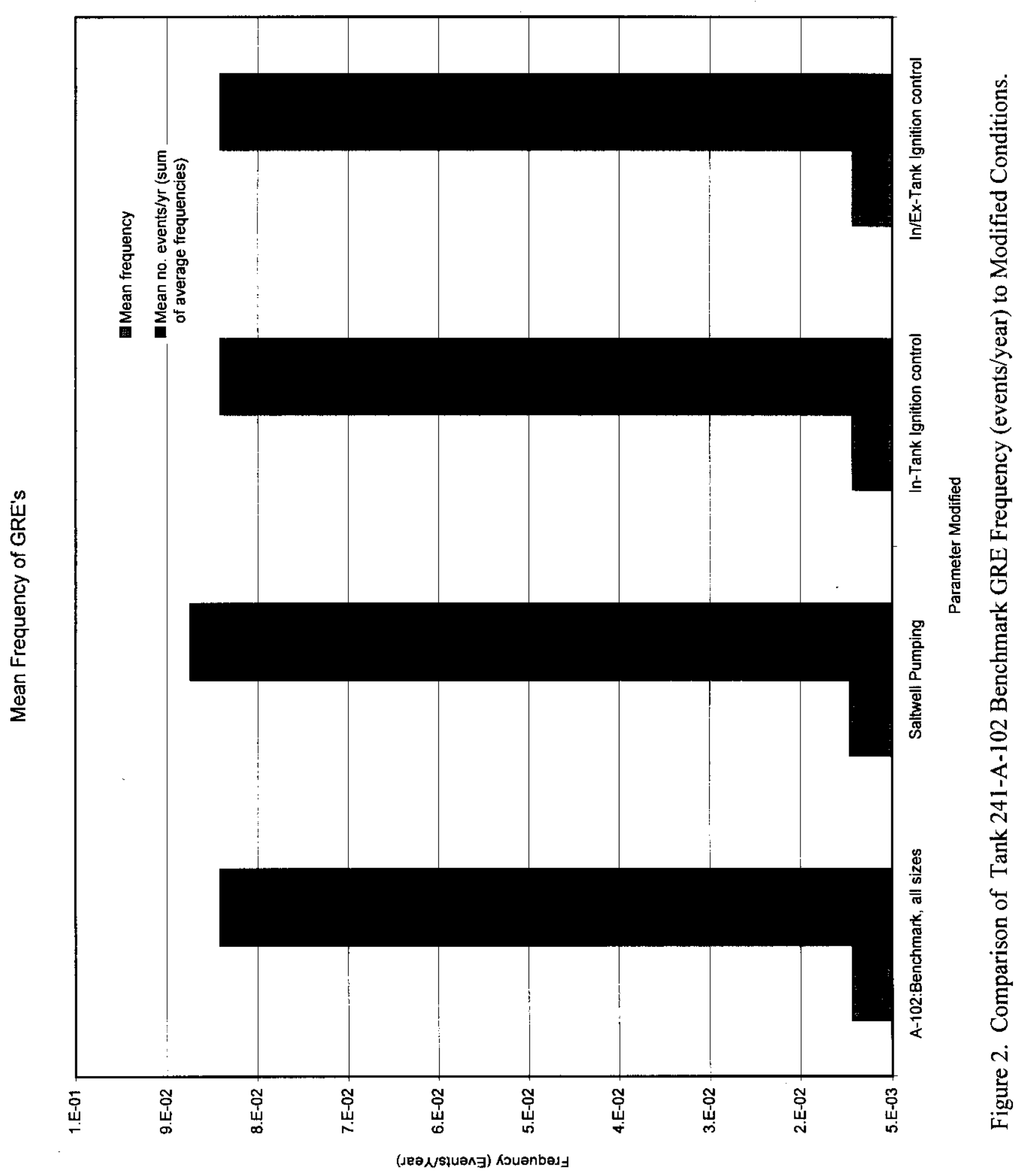


RPP-6888, Rev. 0
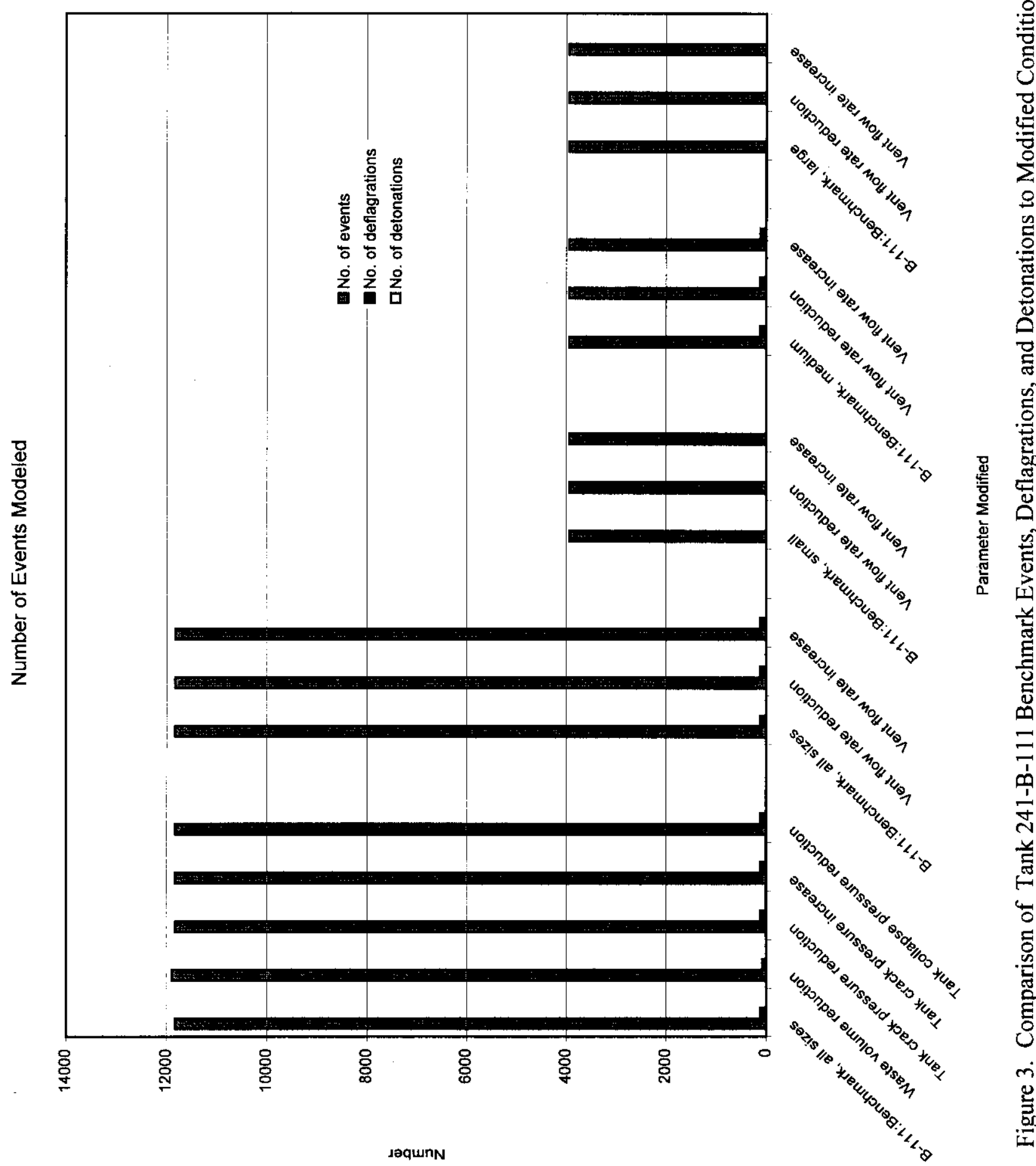

F-3 
RPP-6888, Rev. 0

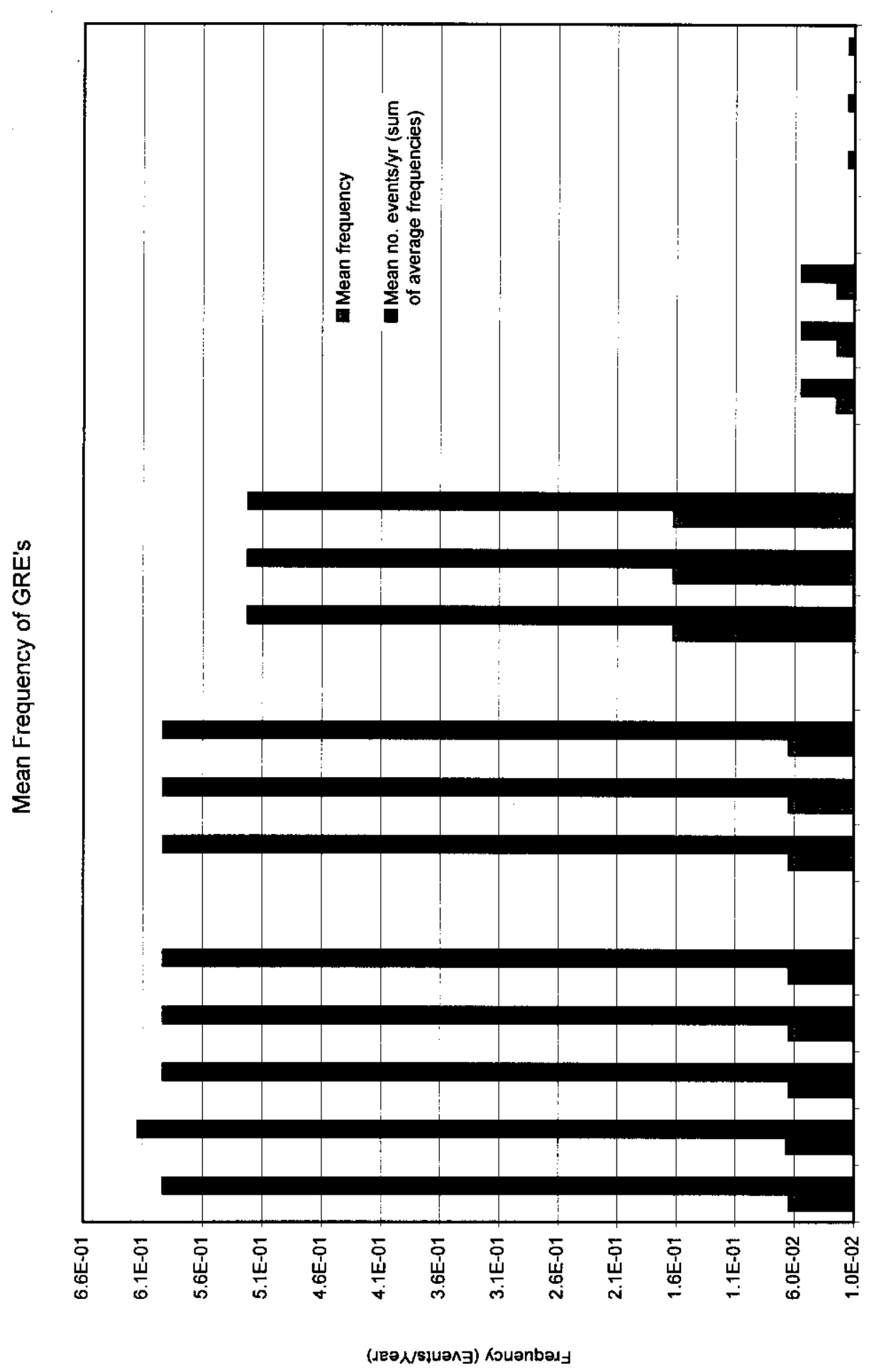

竞 
RPP-6888, Rev. 0

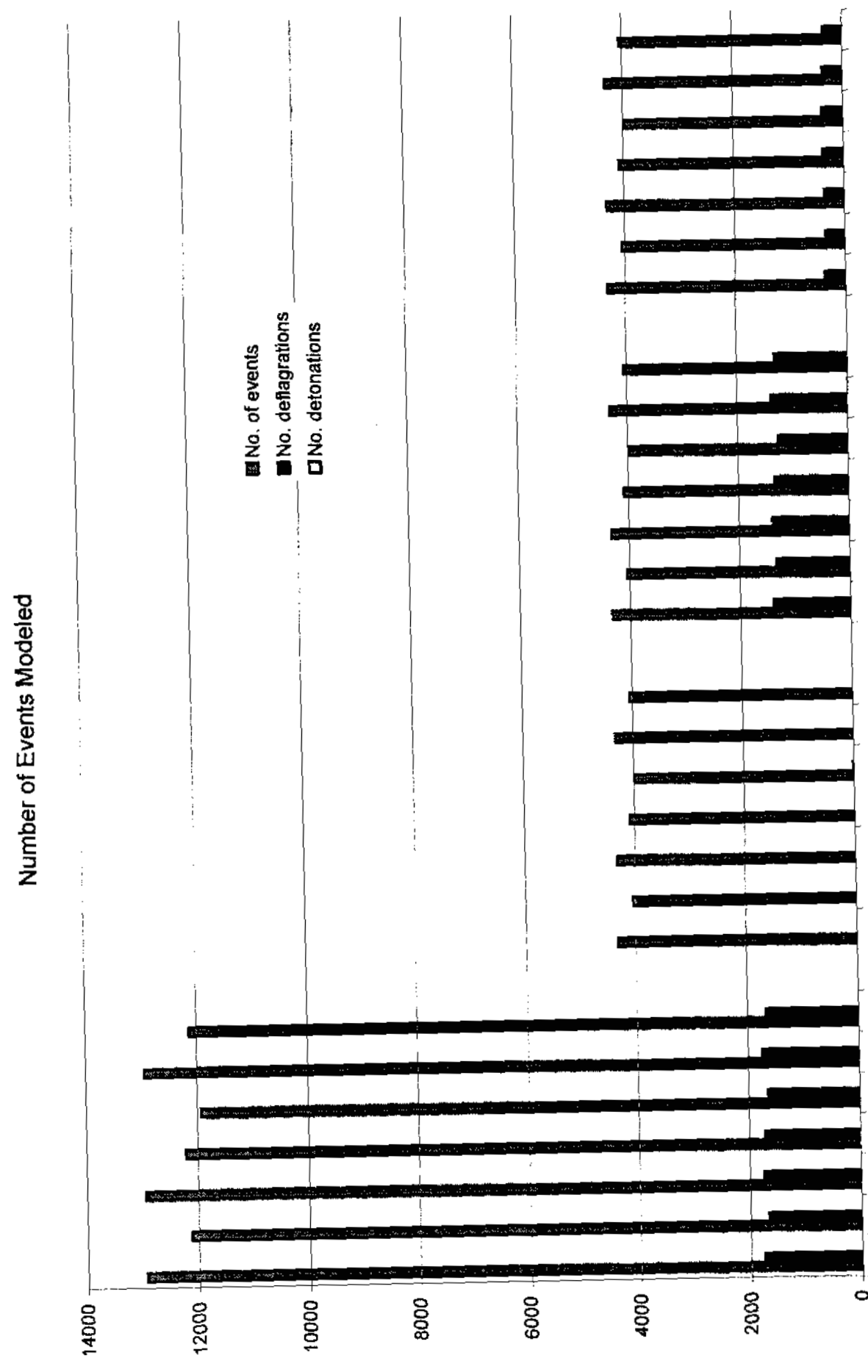

JəqunN

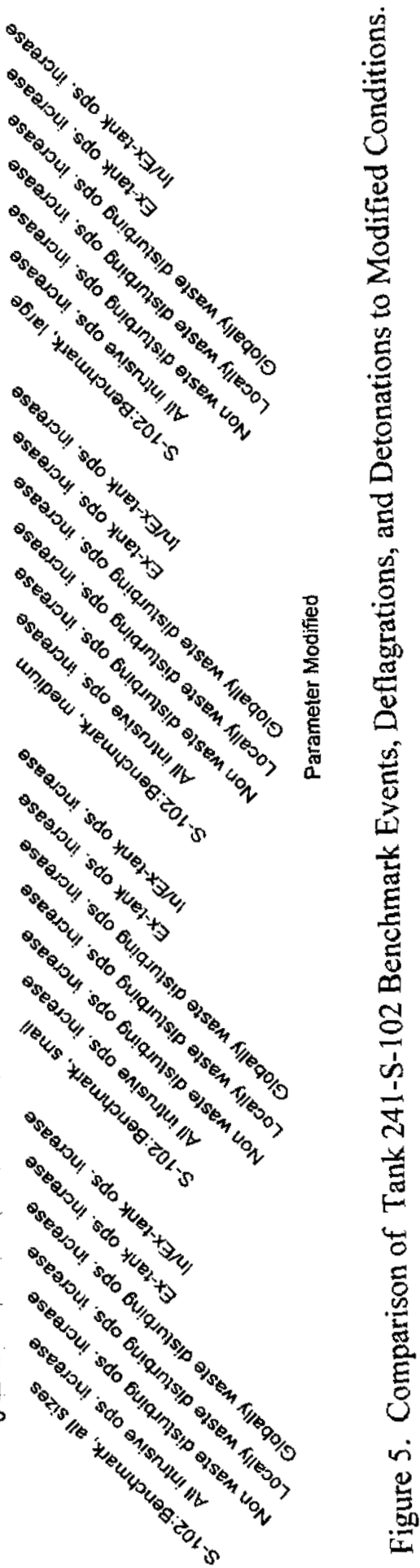


RPP-6888, Rev. 0

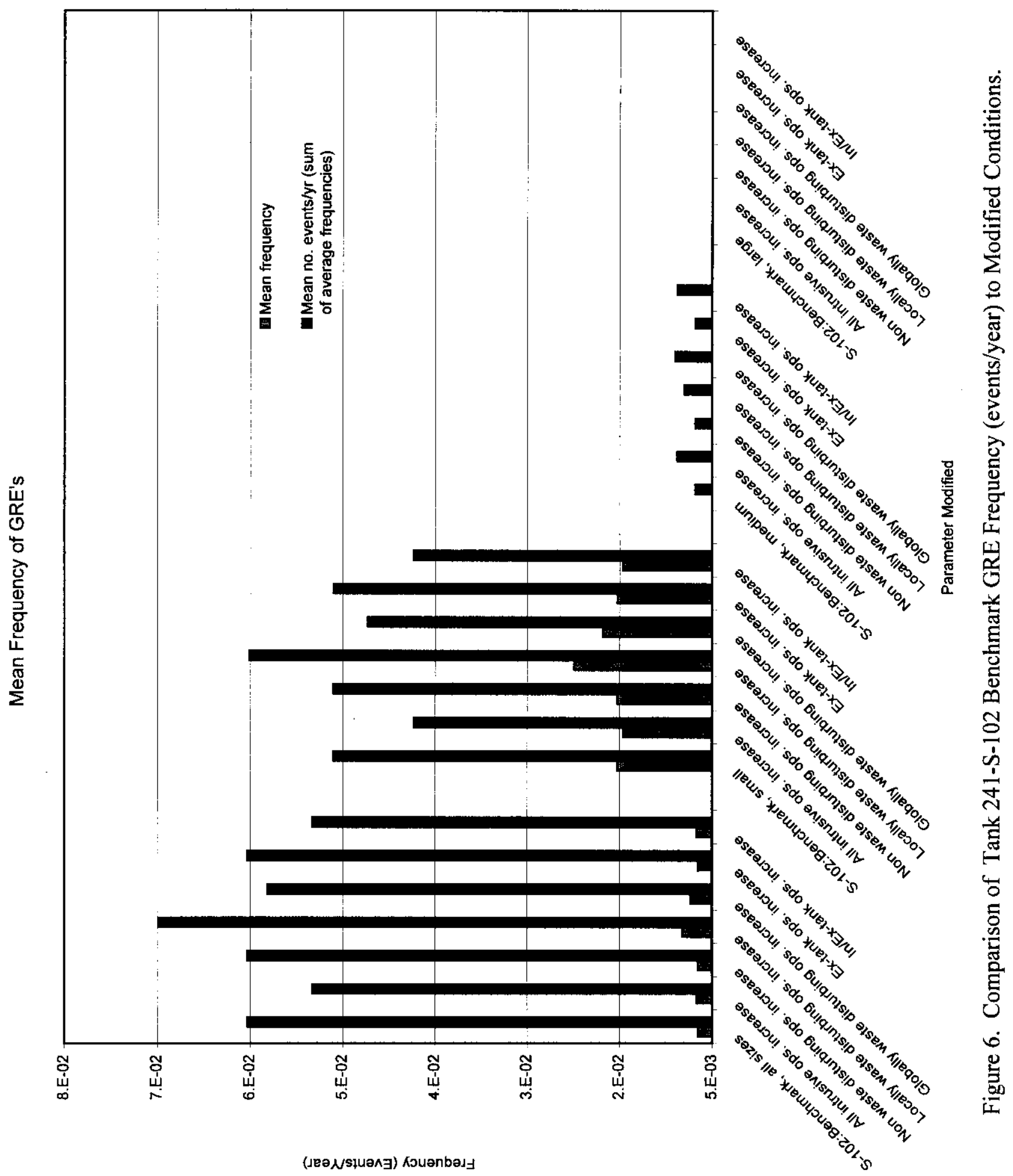


RPP-6888, Rev. 0

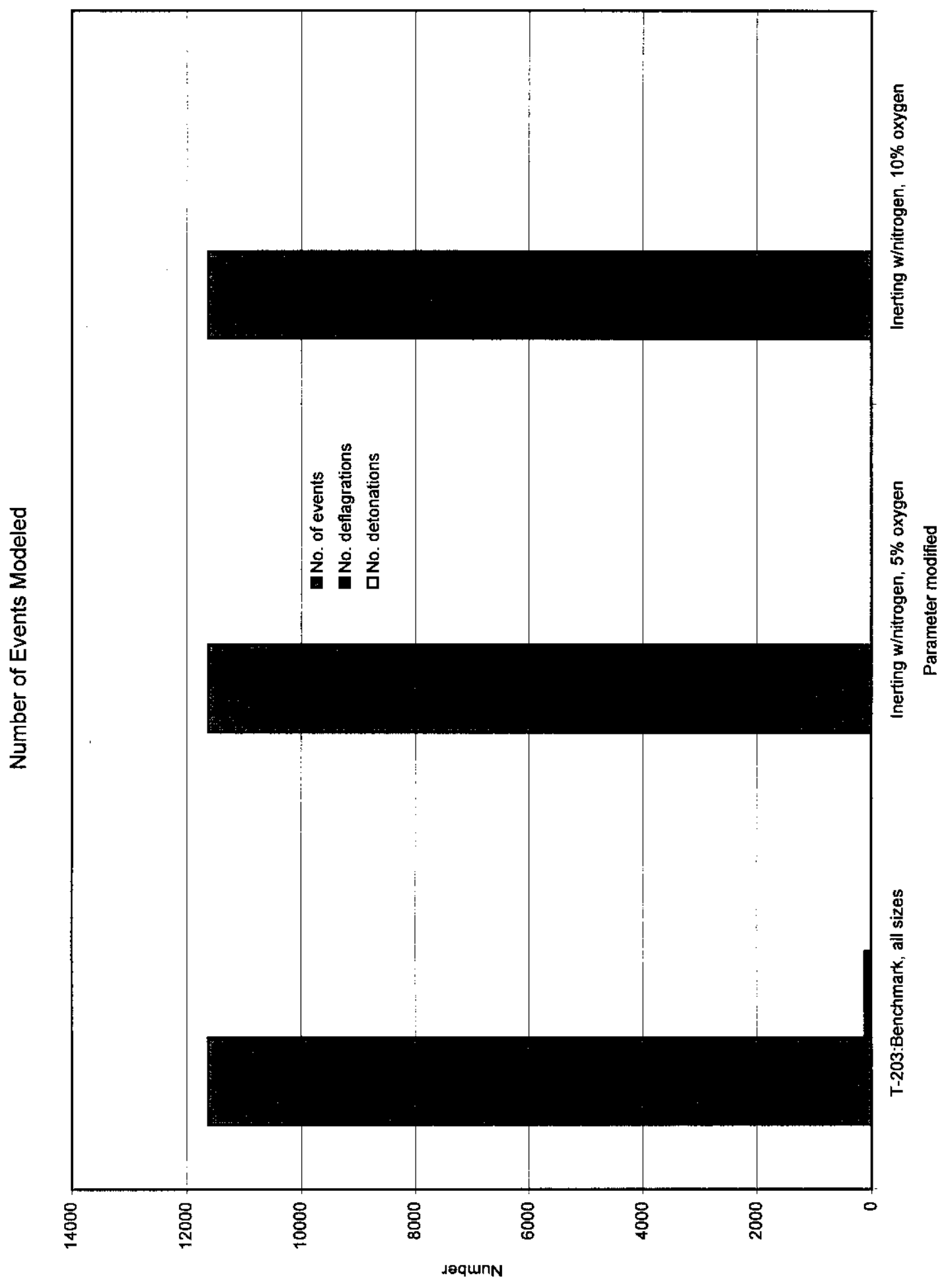

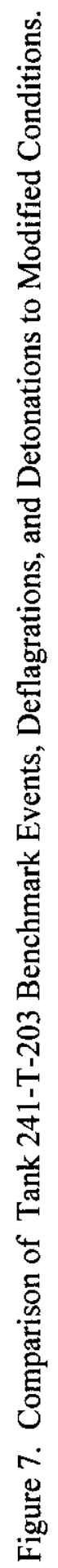


RPP-6888, Rev. 0

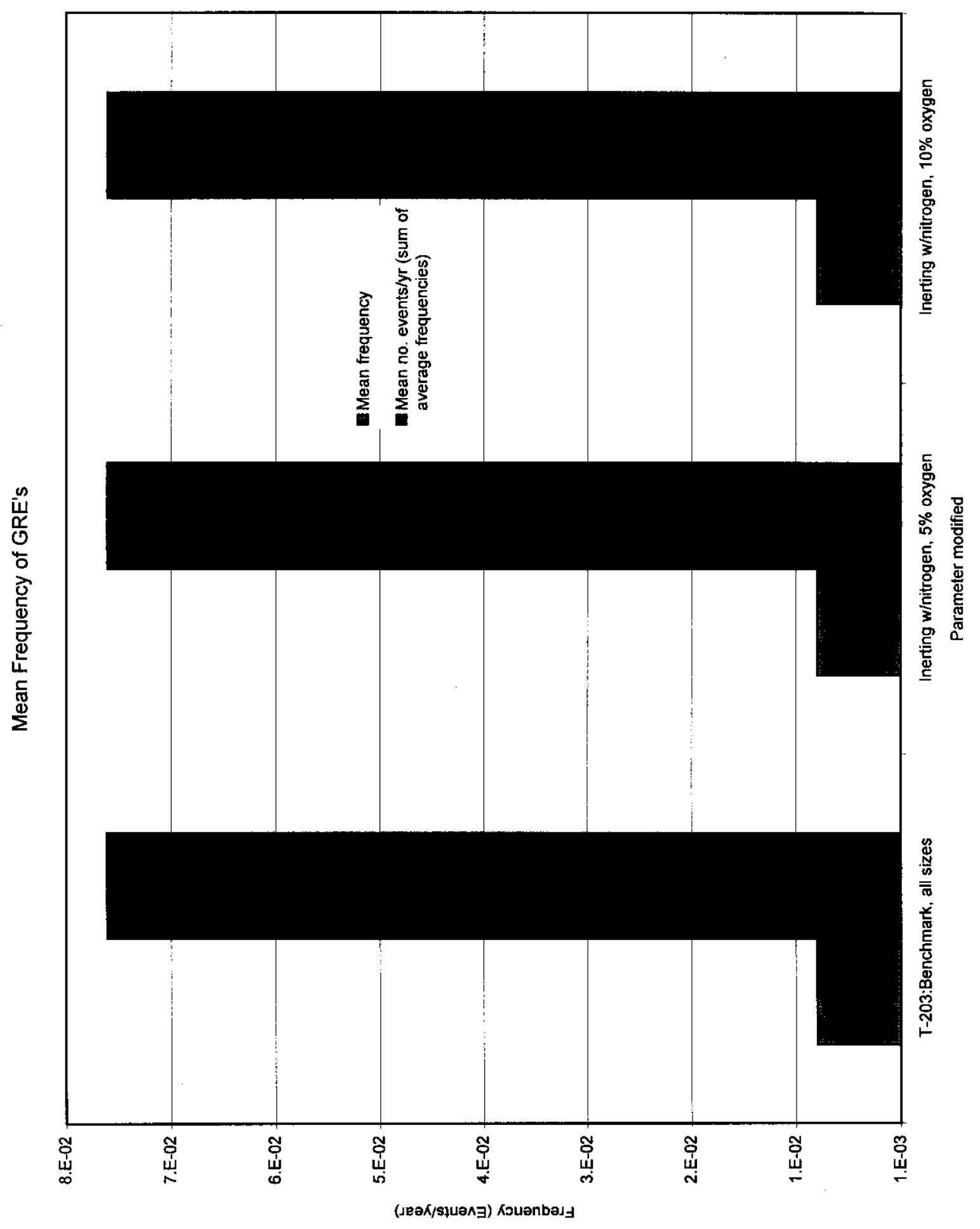

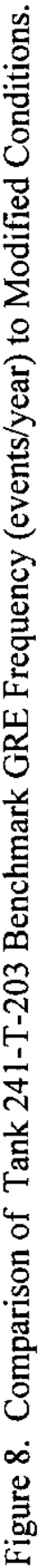


RPP-6888, Rev. 0

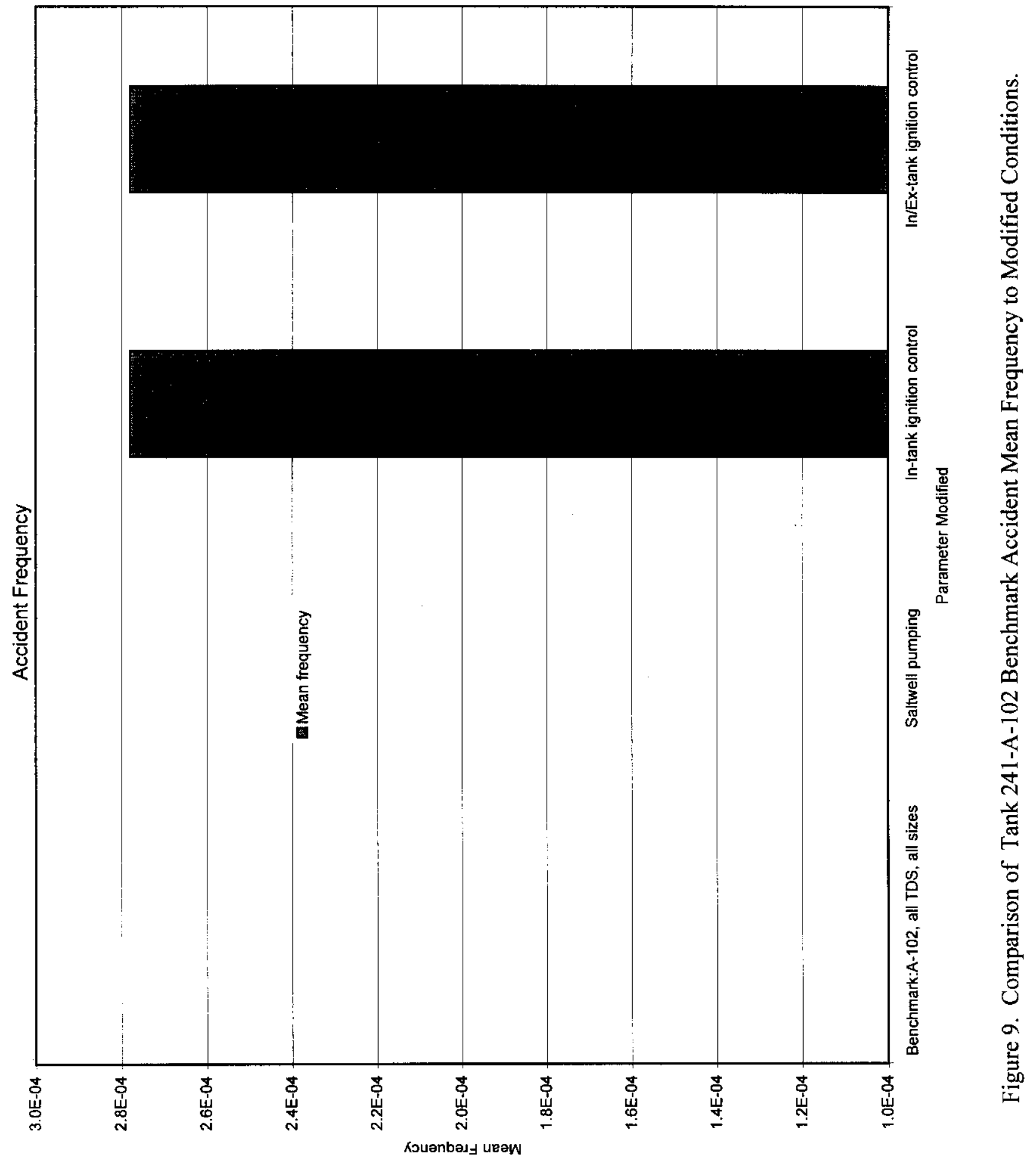

F-9 
RPP-6888, Rev. 0

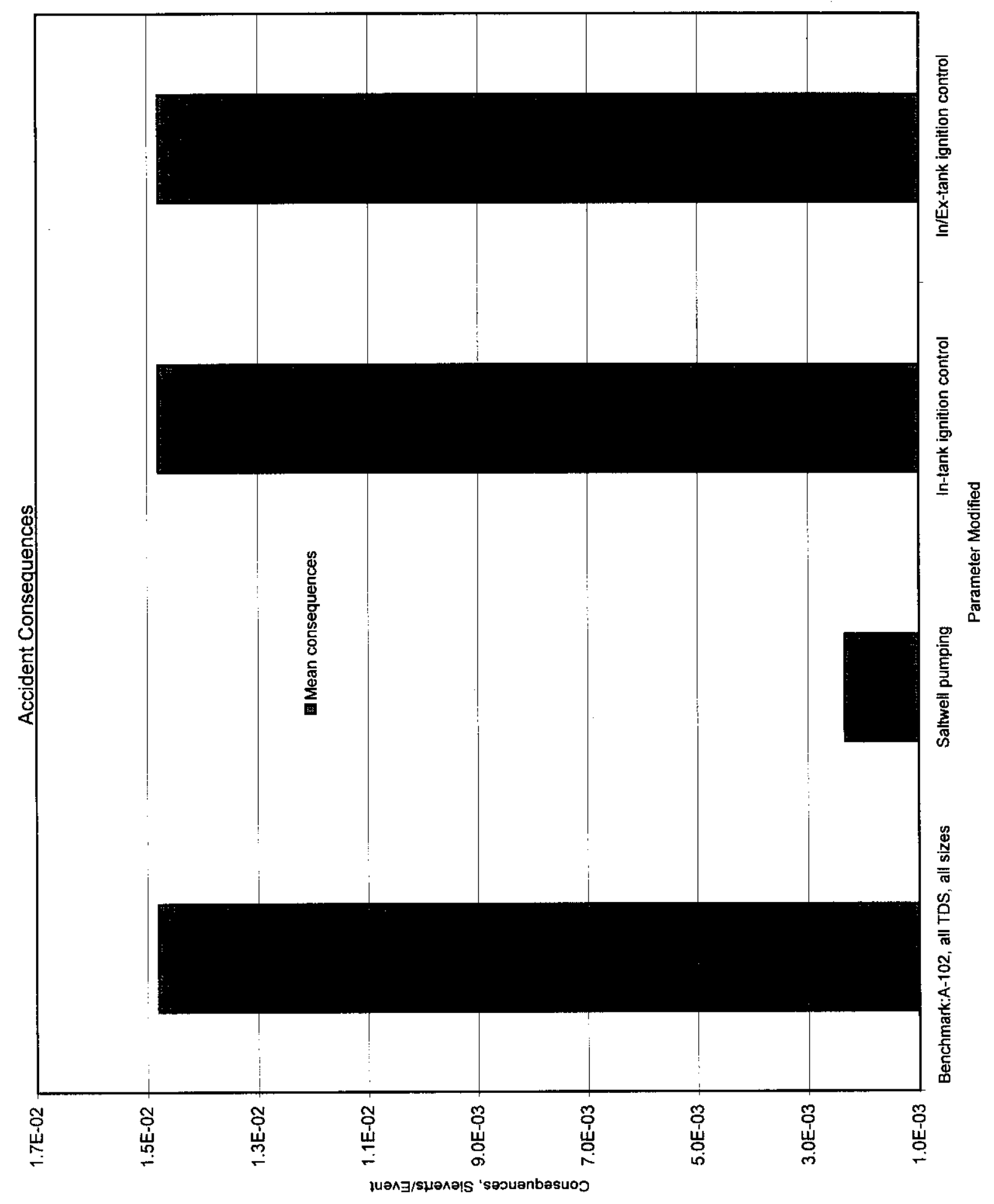

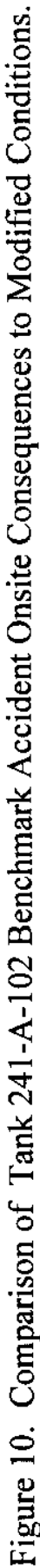

F-10 
RPP-6888, Rev. 0

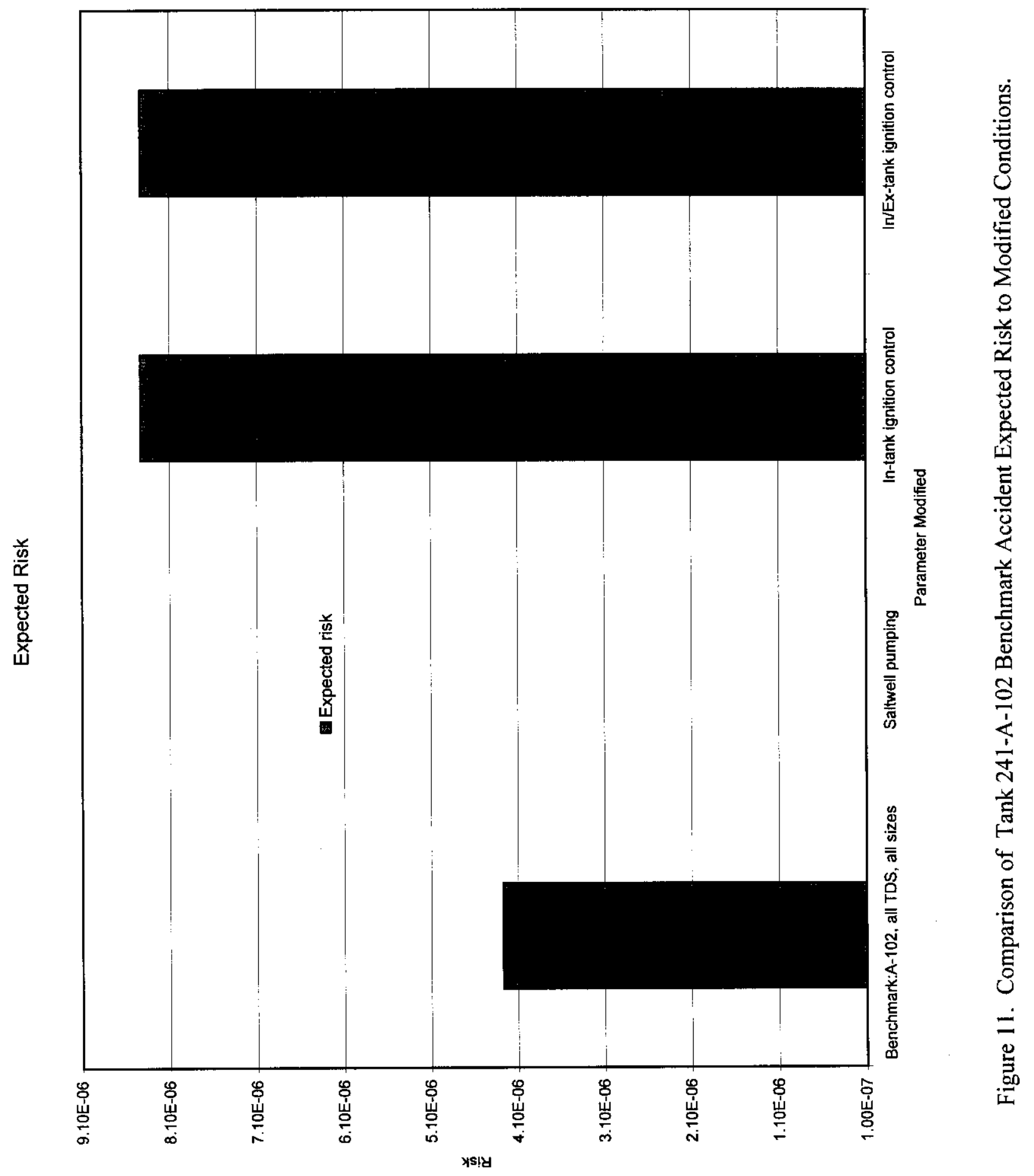

F-11 
RPP-6888, Rev. 0
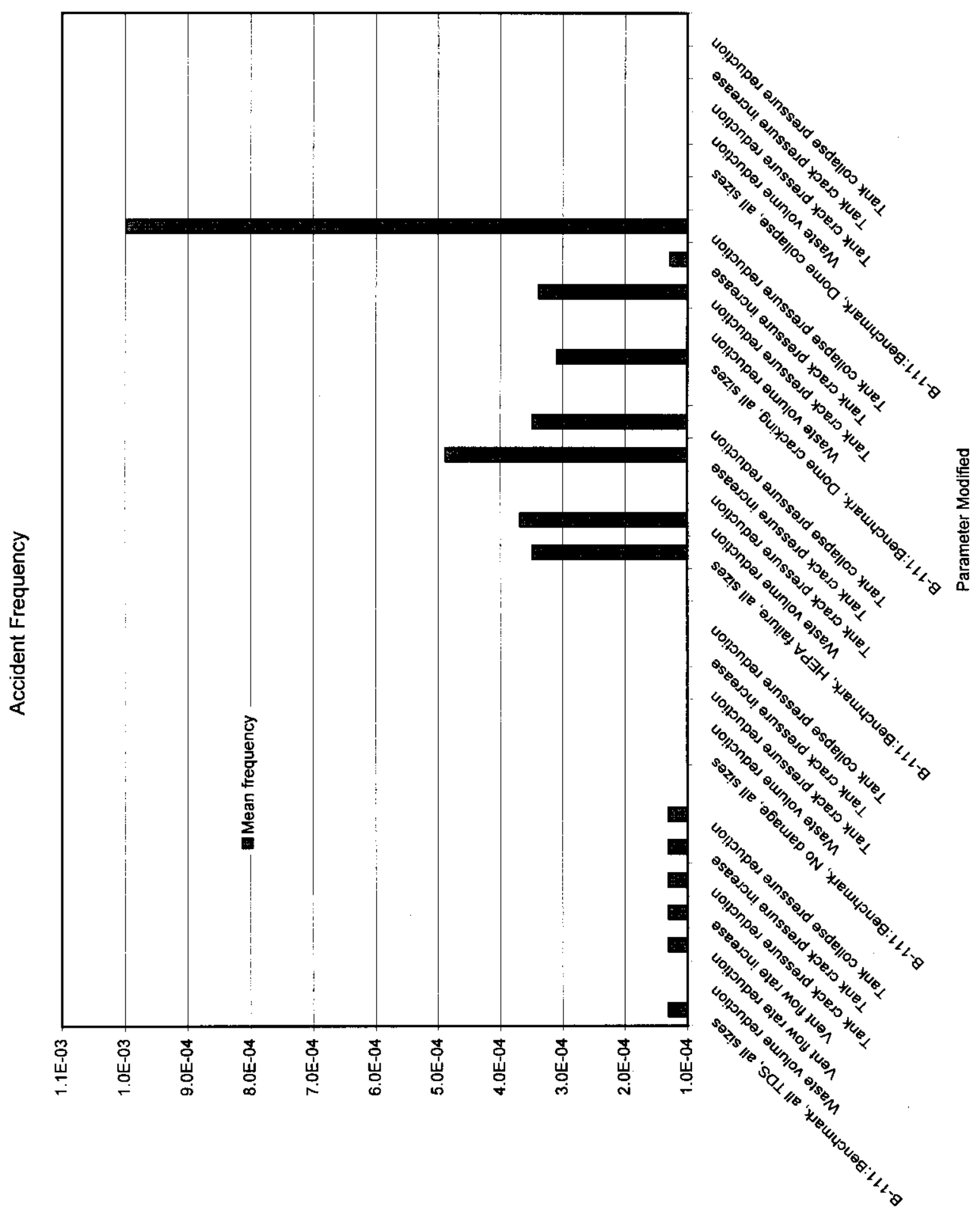

КวuənbอJ 
RPP-6888, Rev. 0
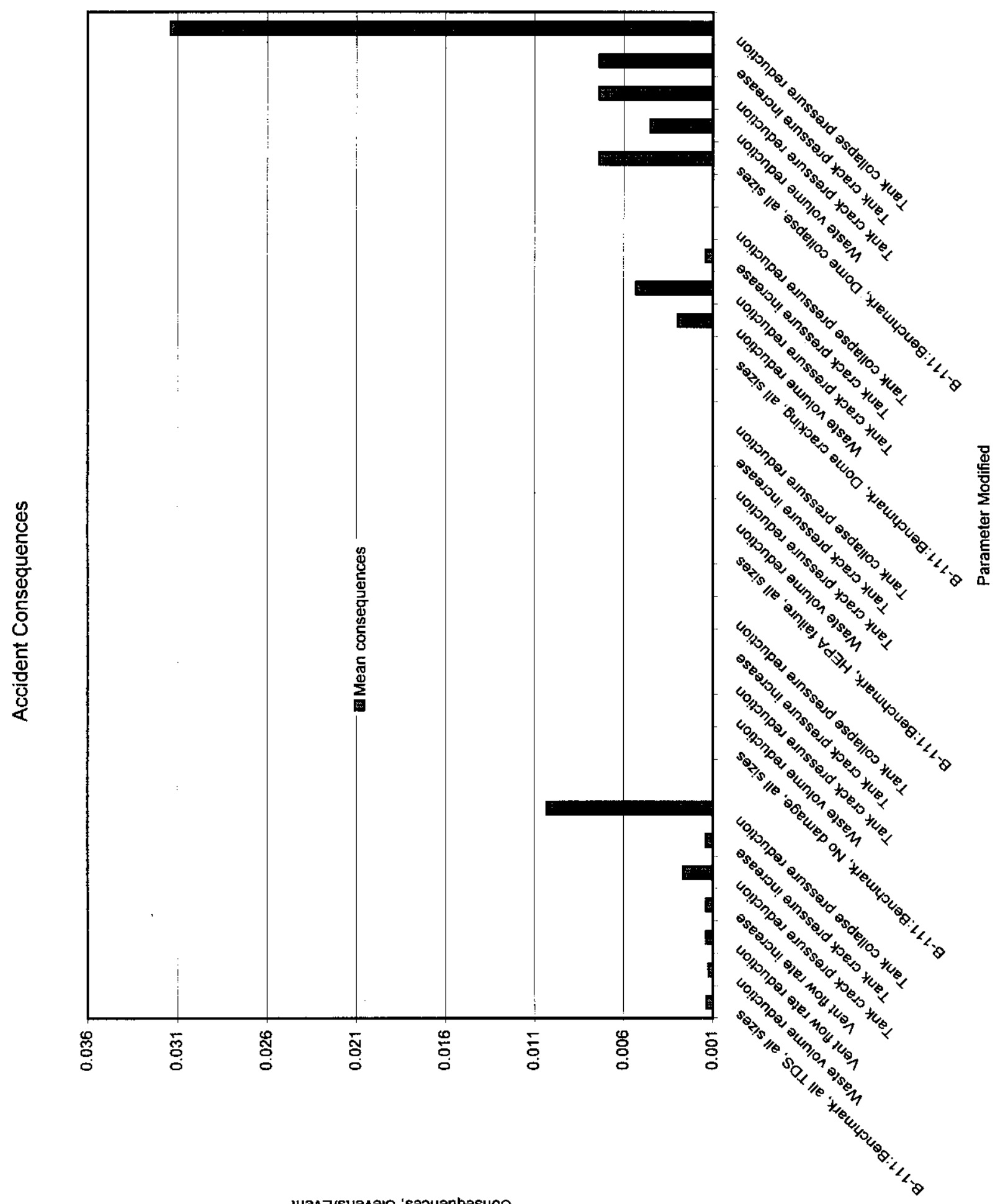
RPP-6888, Rev. 0

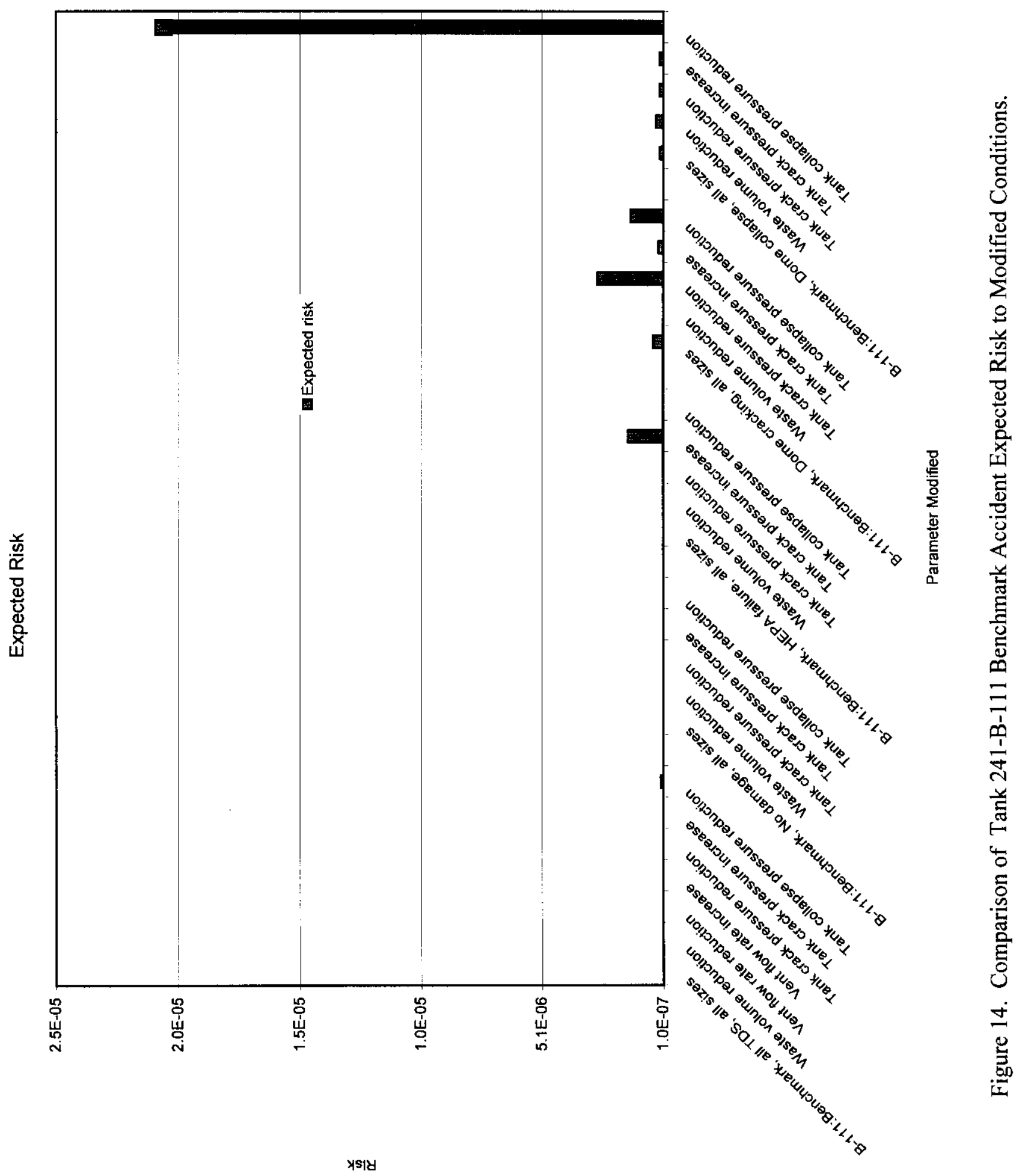


RPP-6888, Rev. 0



F-15 
RPP-6888, Rev. 0

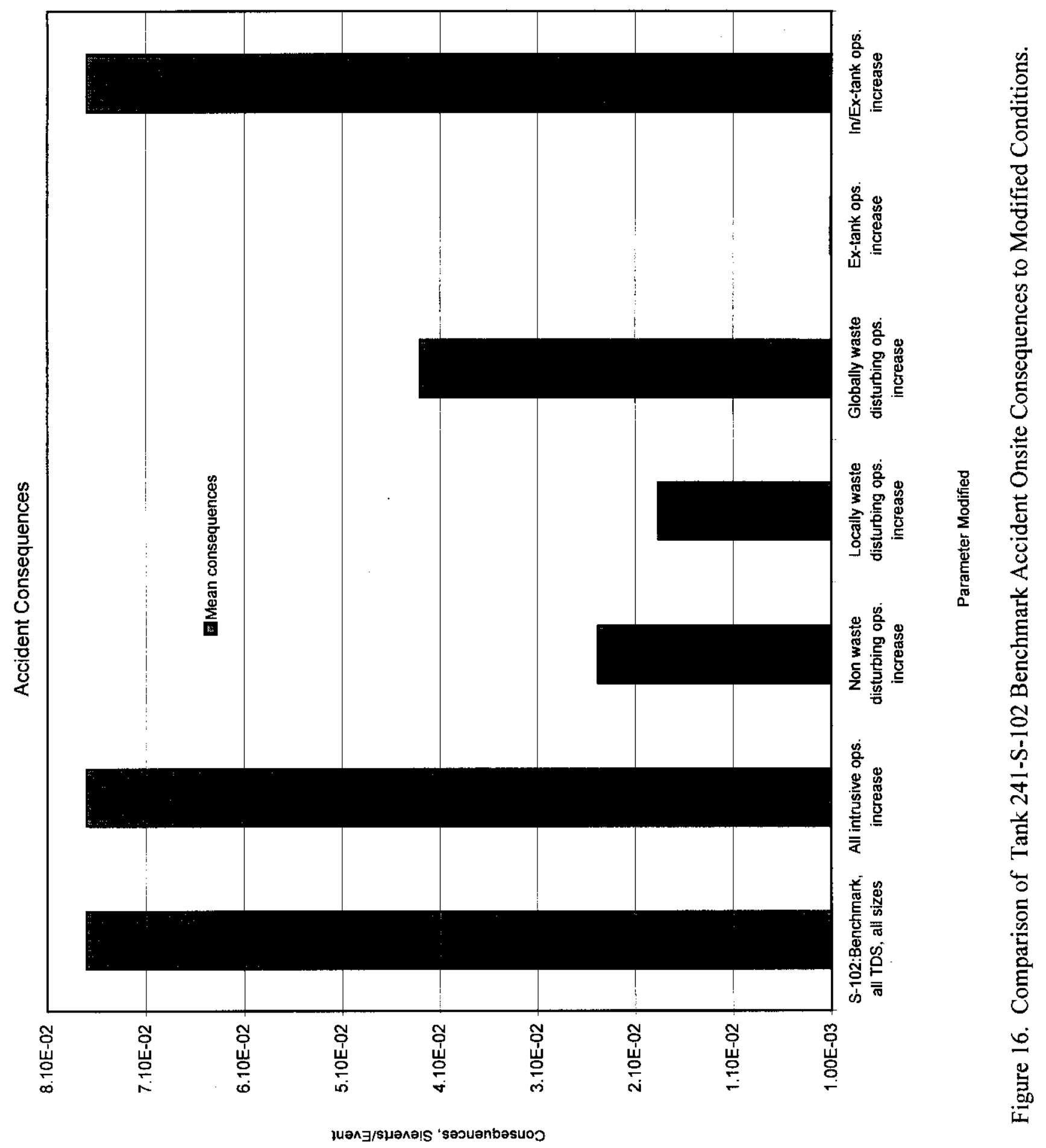

F-16 
RPP-6888, Rev. 0

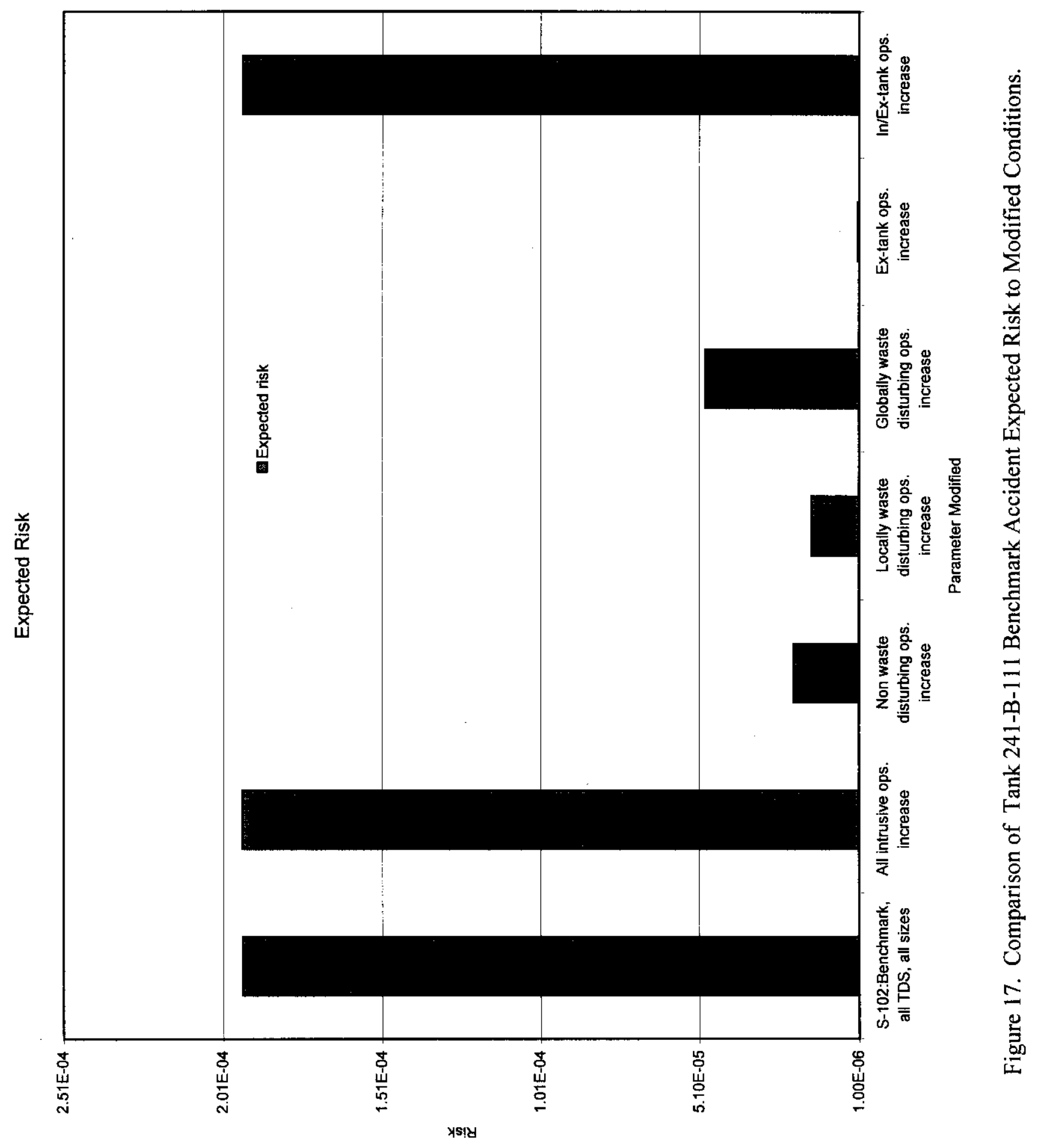

F-17 
RPP-6888, Rev. 0

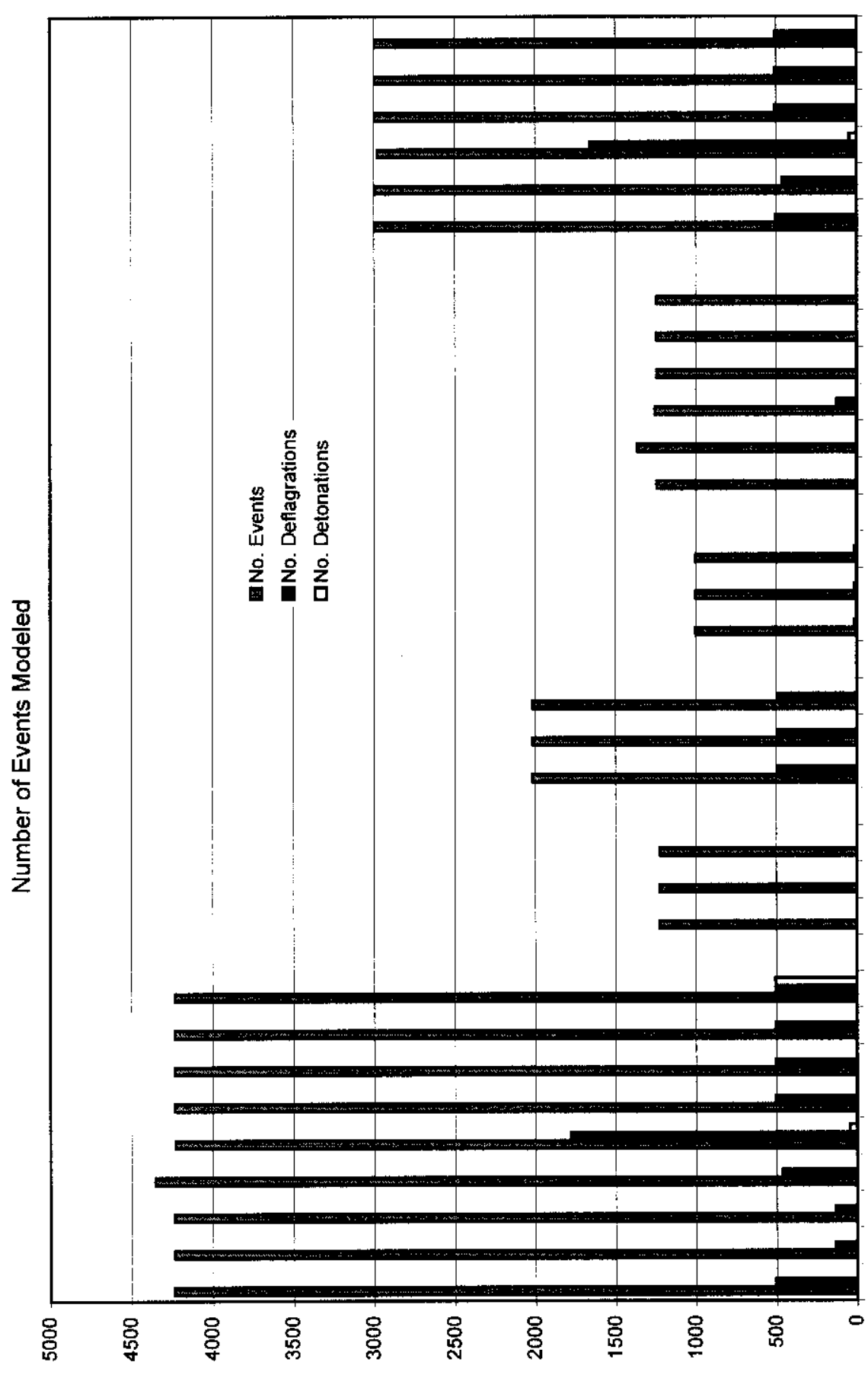

JəqunN

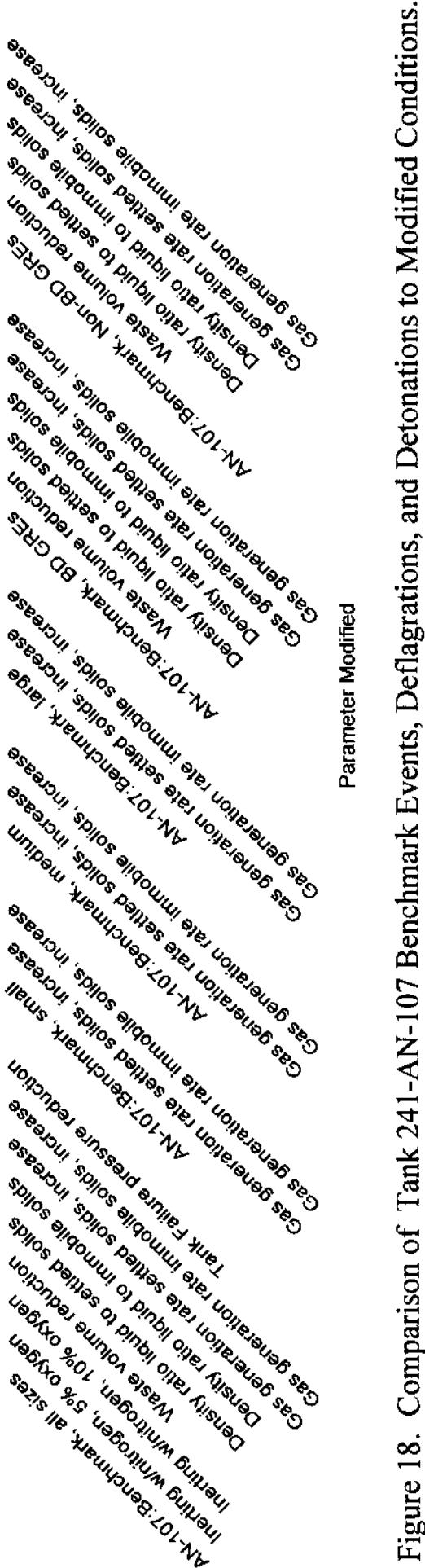


RPP-6888, Rev. 0
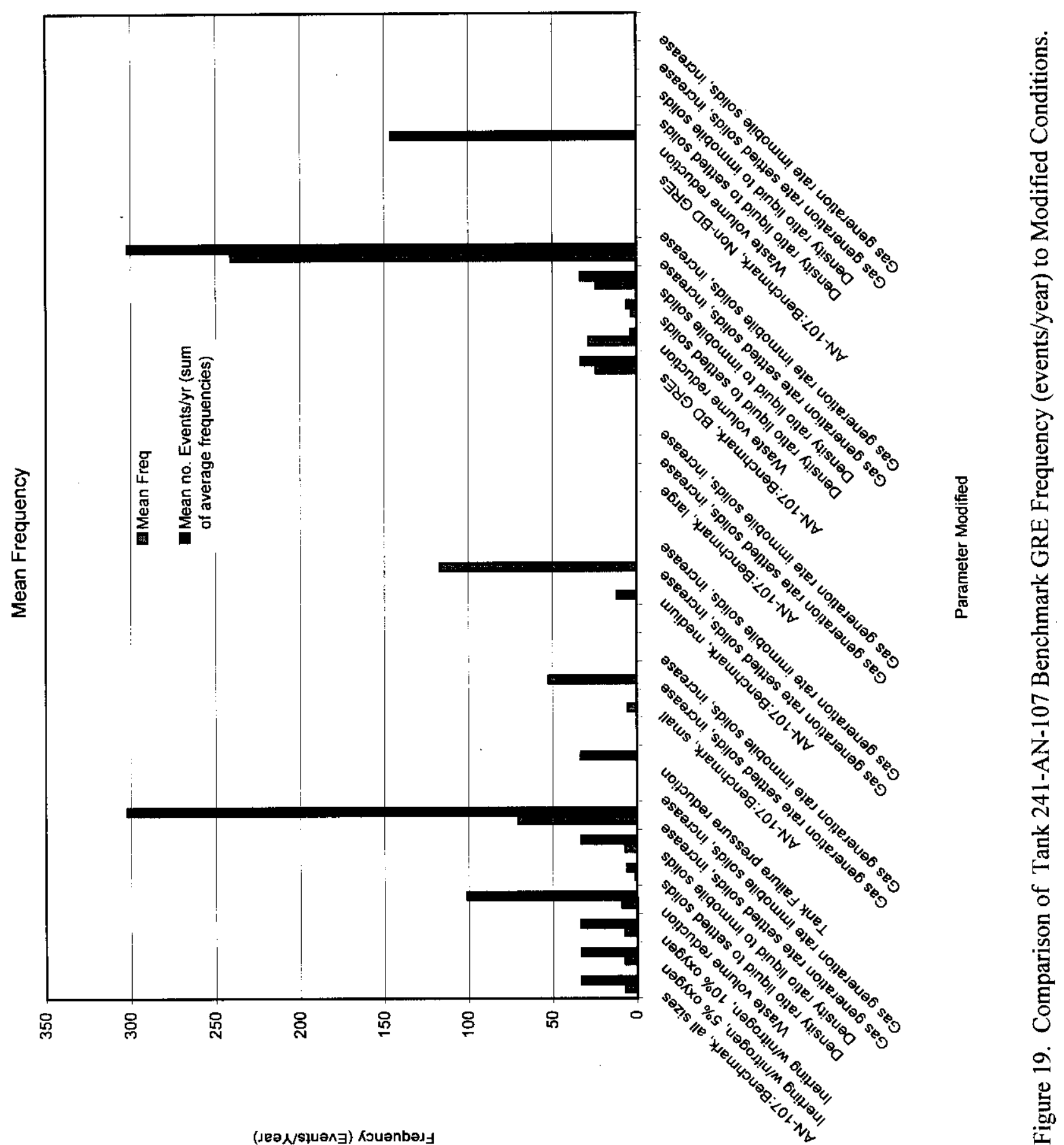
RPP-6888, Rev. 0

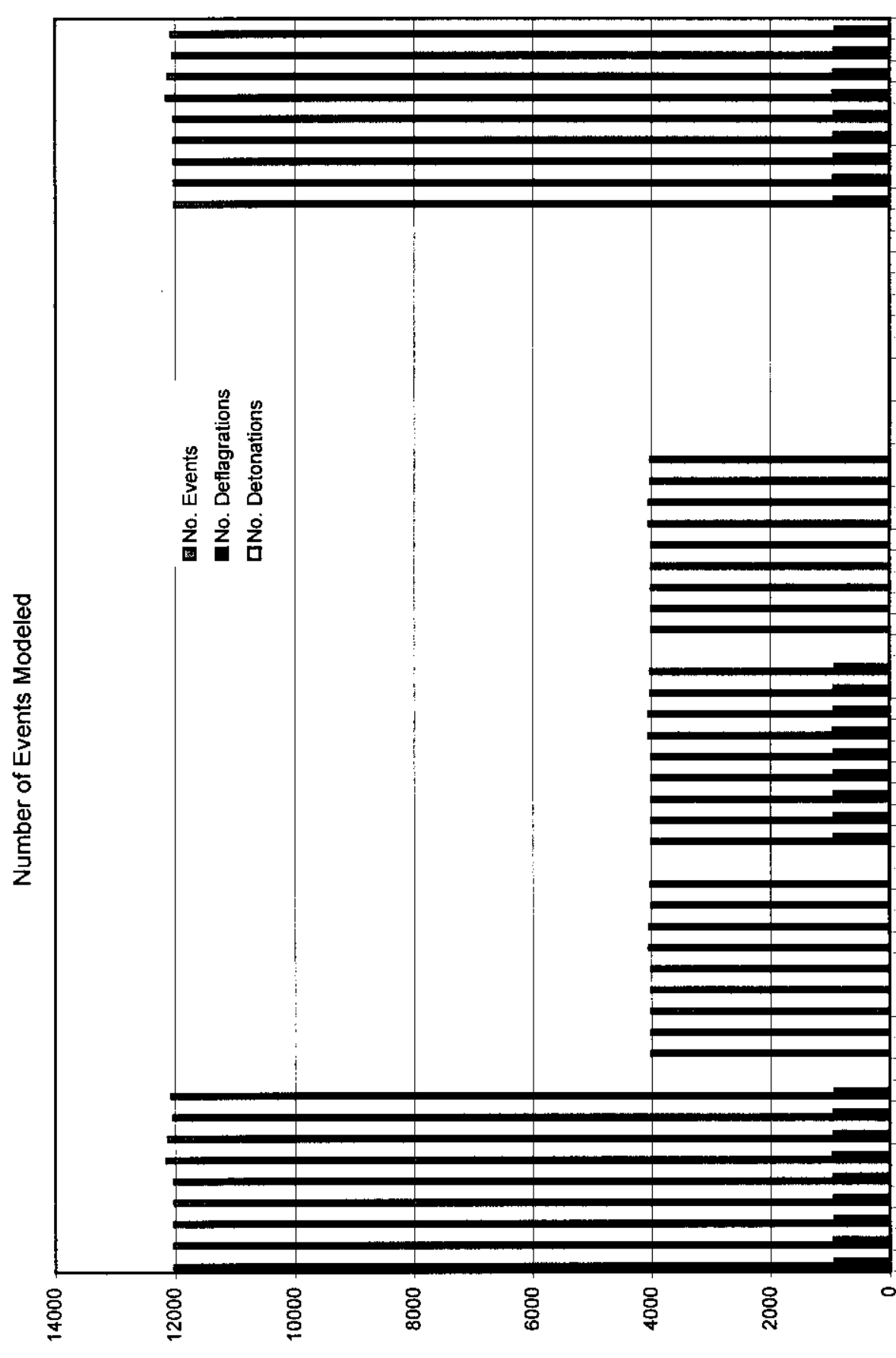

sequnN

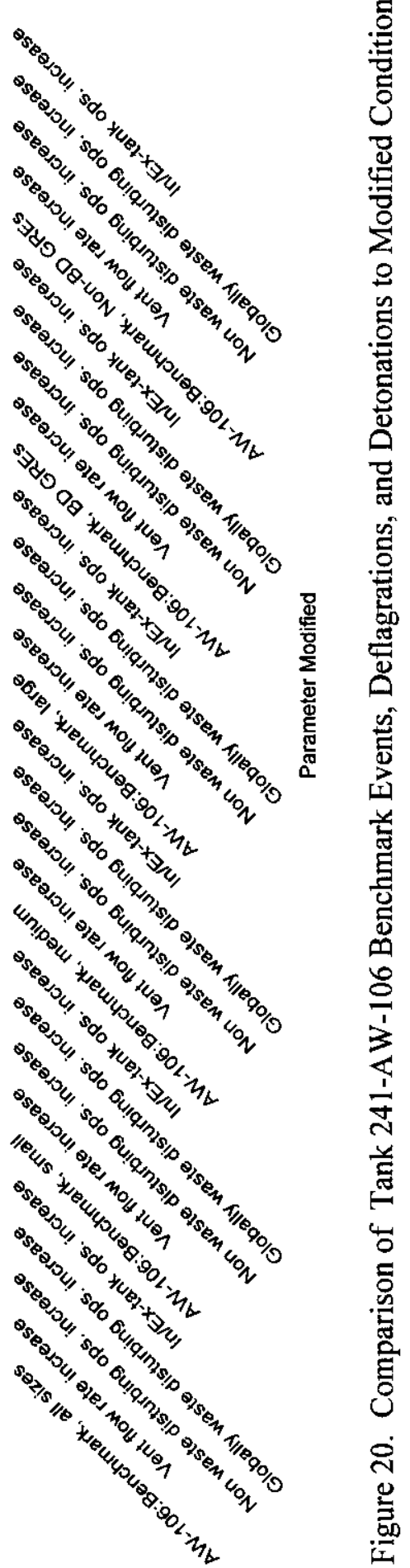


RPP-6888, Rev. 0
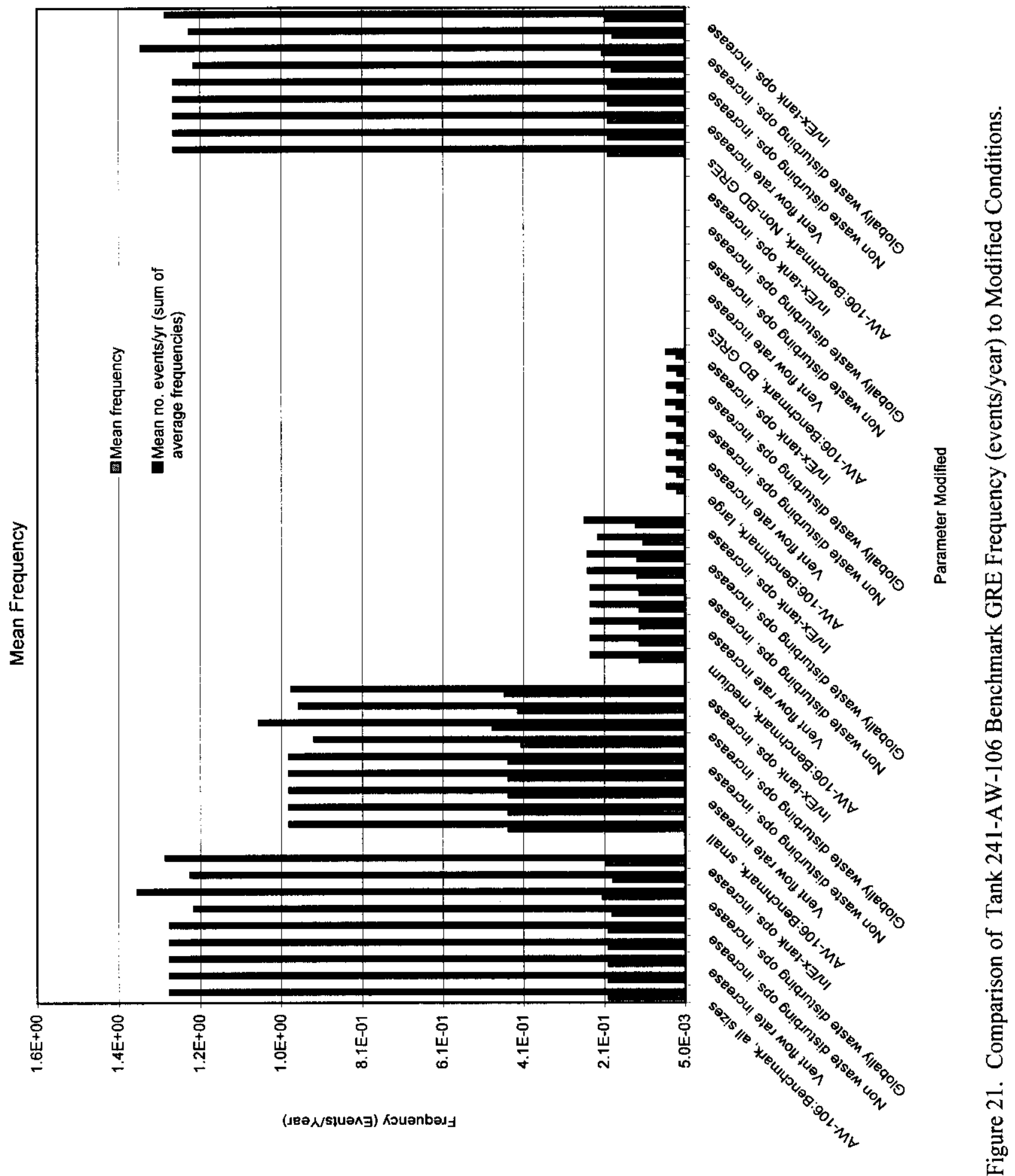
RPP-6888, Rev. 0

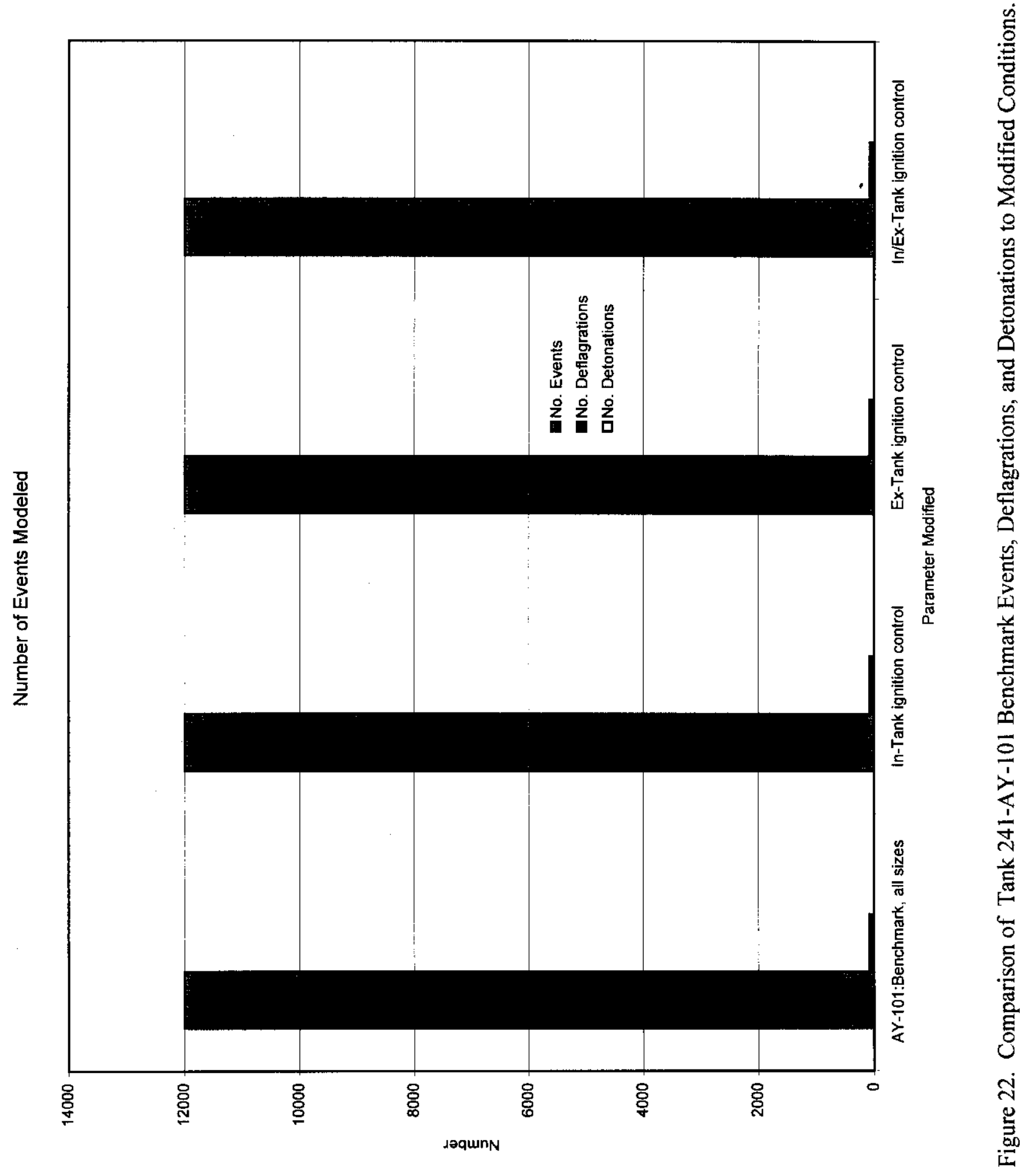


RPP-6888, Rev. 0

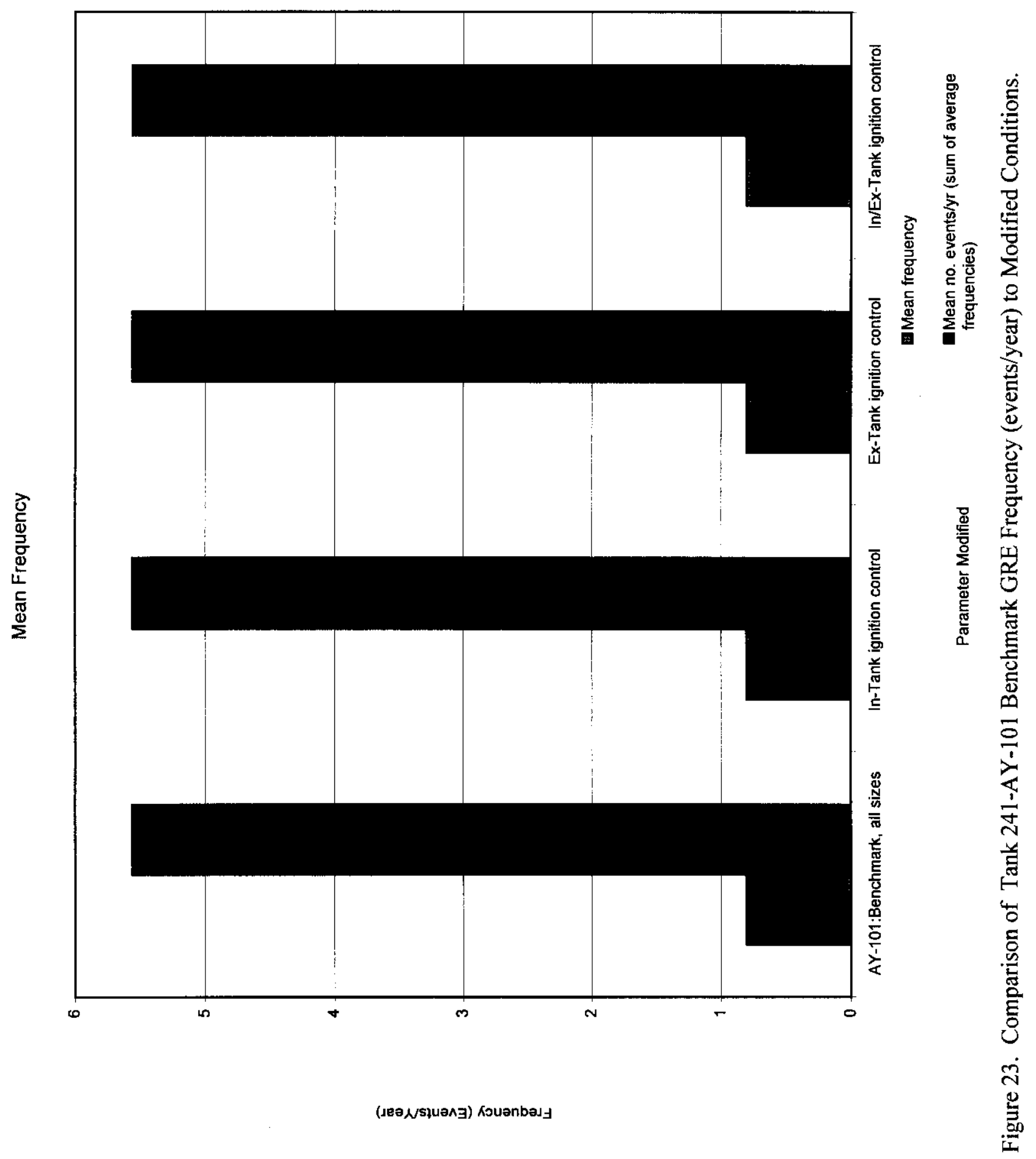


RPP-6888, Rev. 0

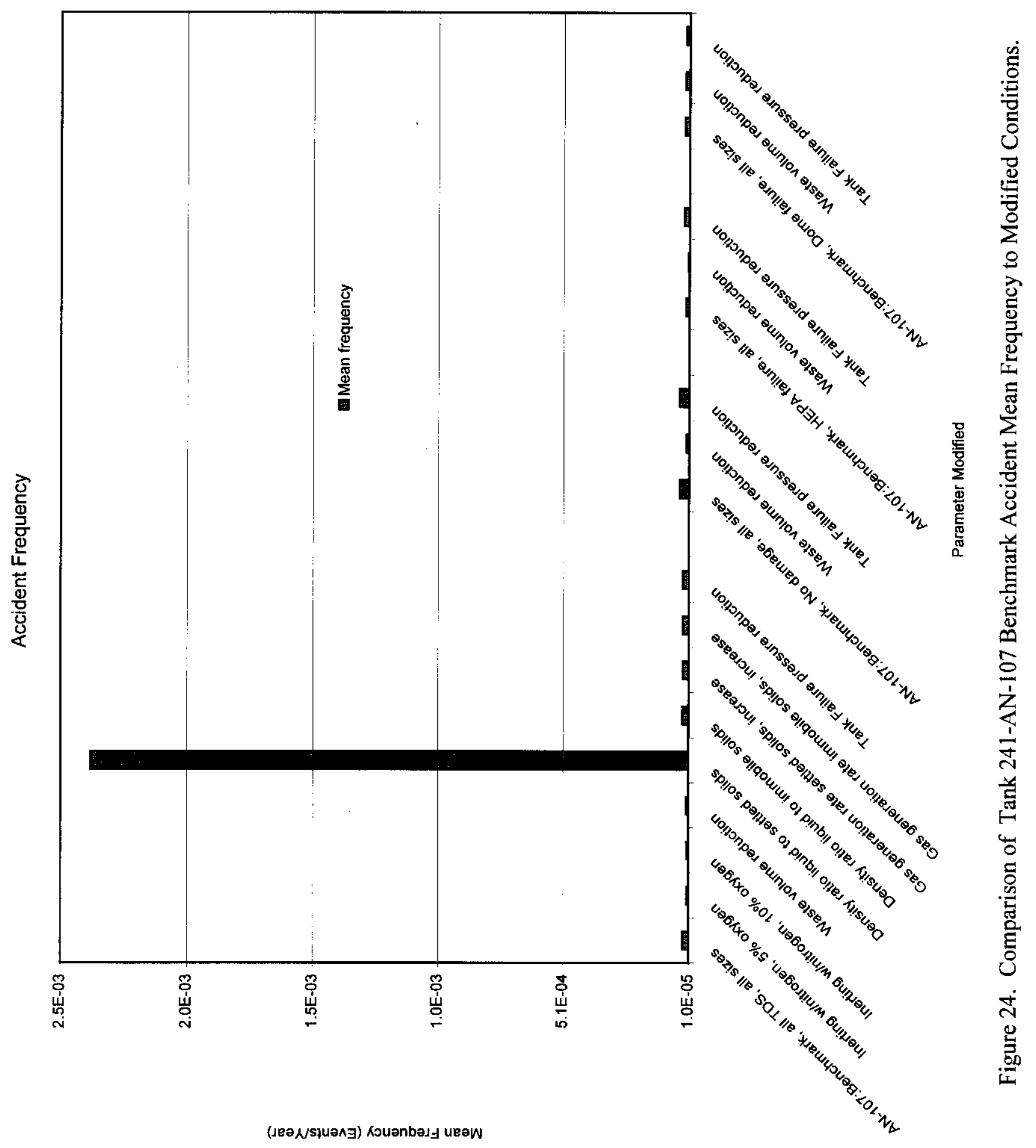


RPP-6888, Rev. 0

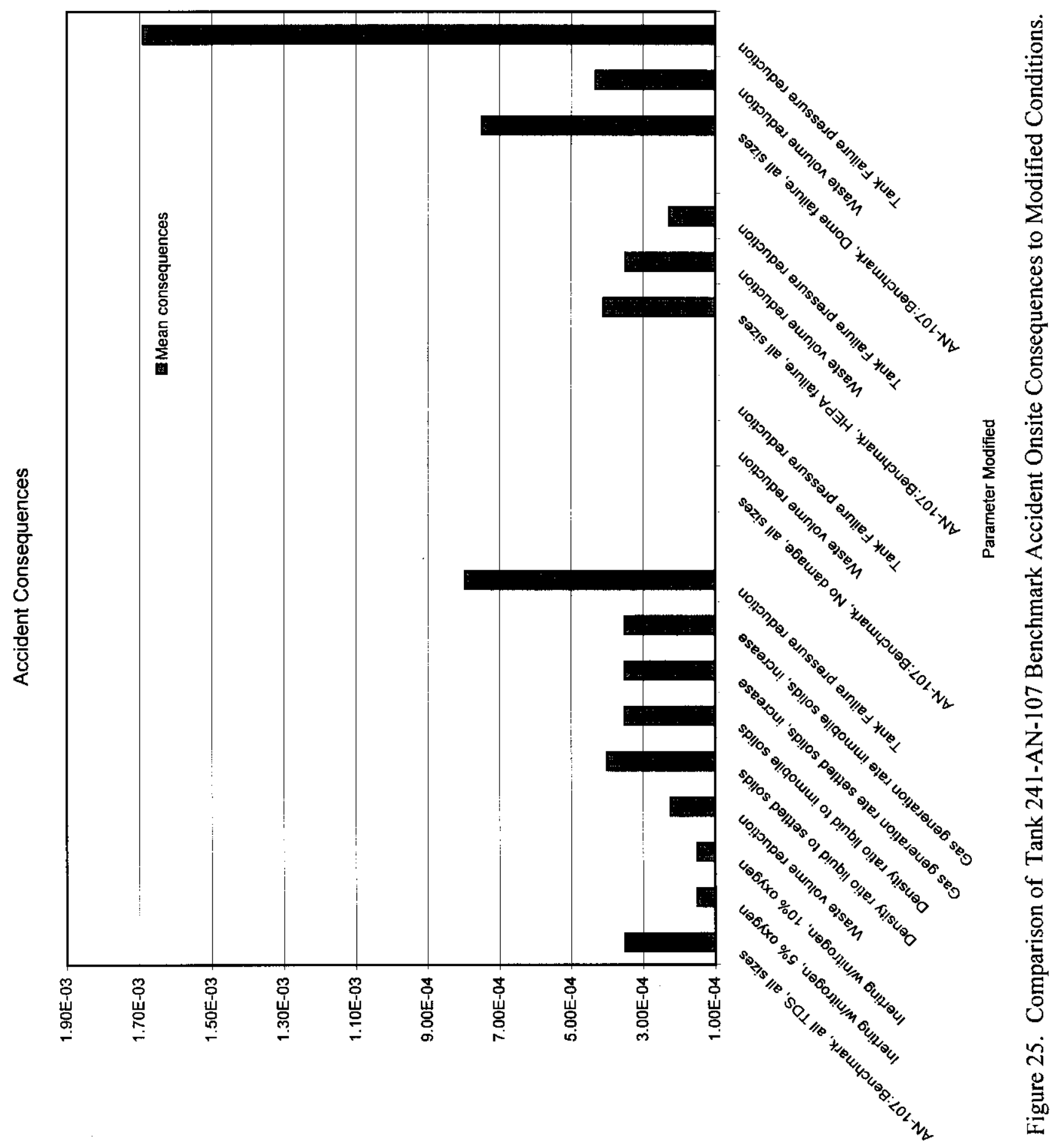

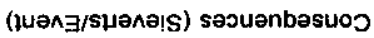


RPP-6888, Rev. 0
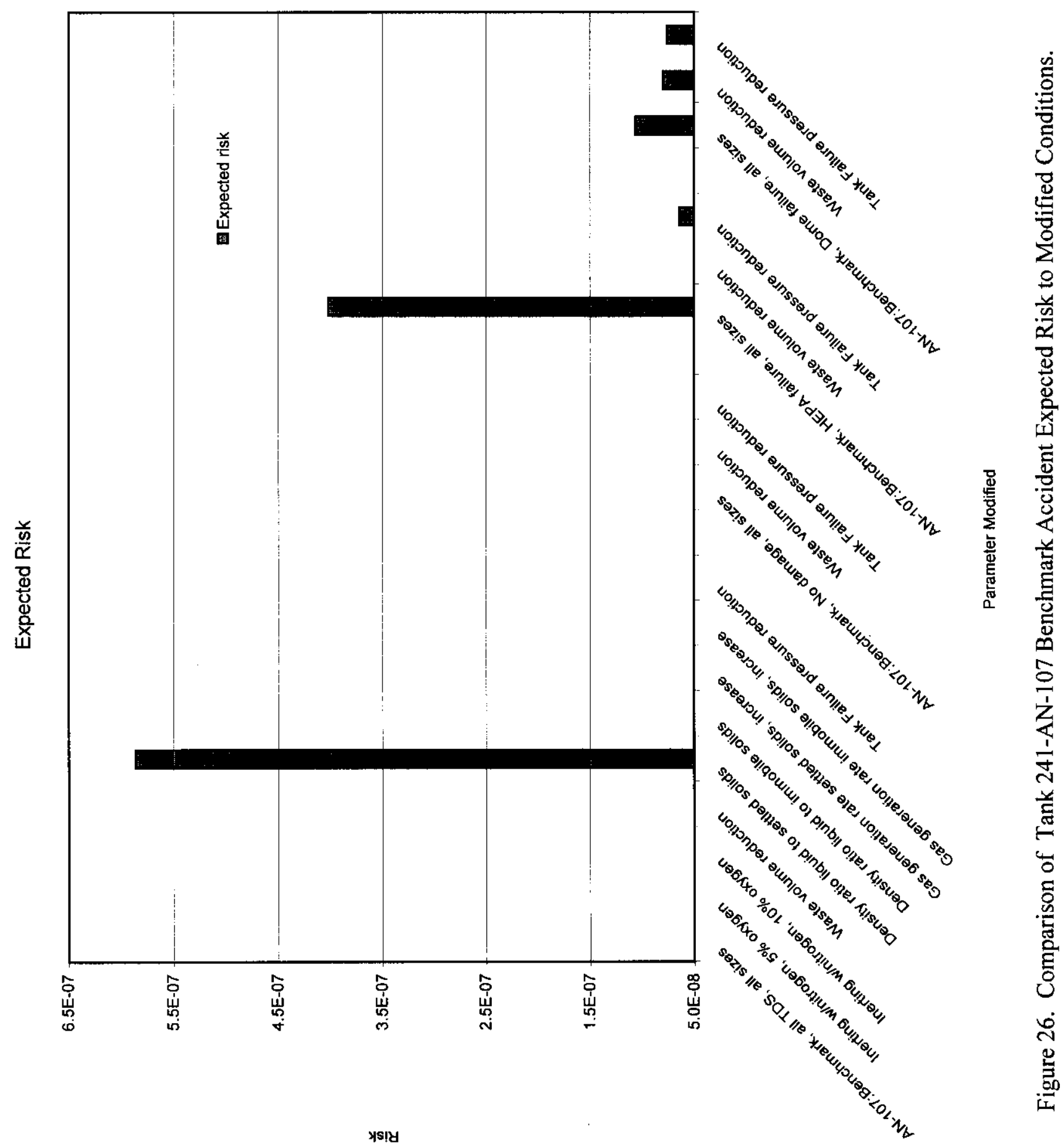
RPP-6888, Rev. 0

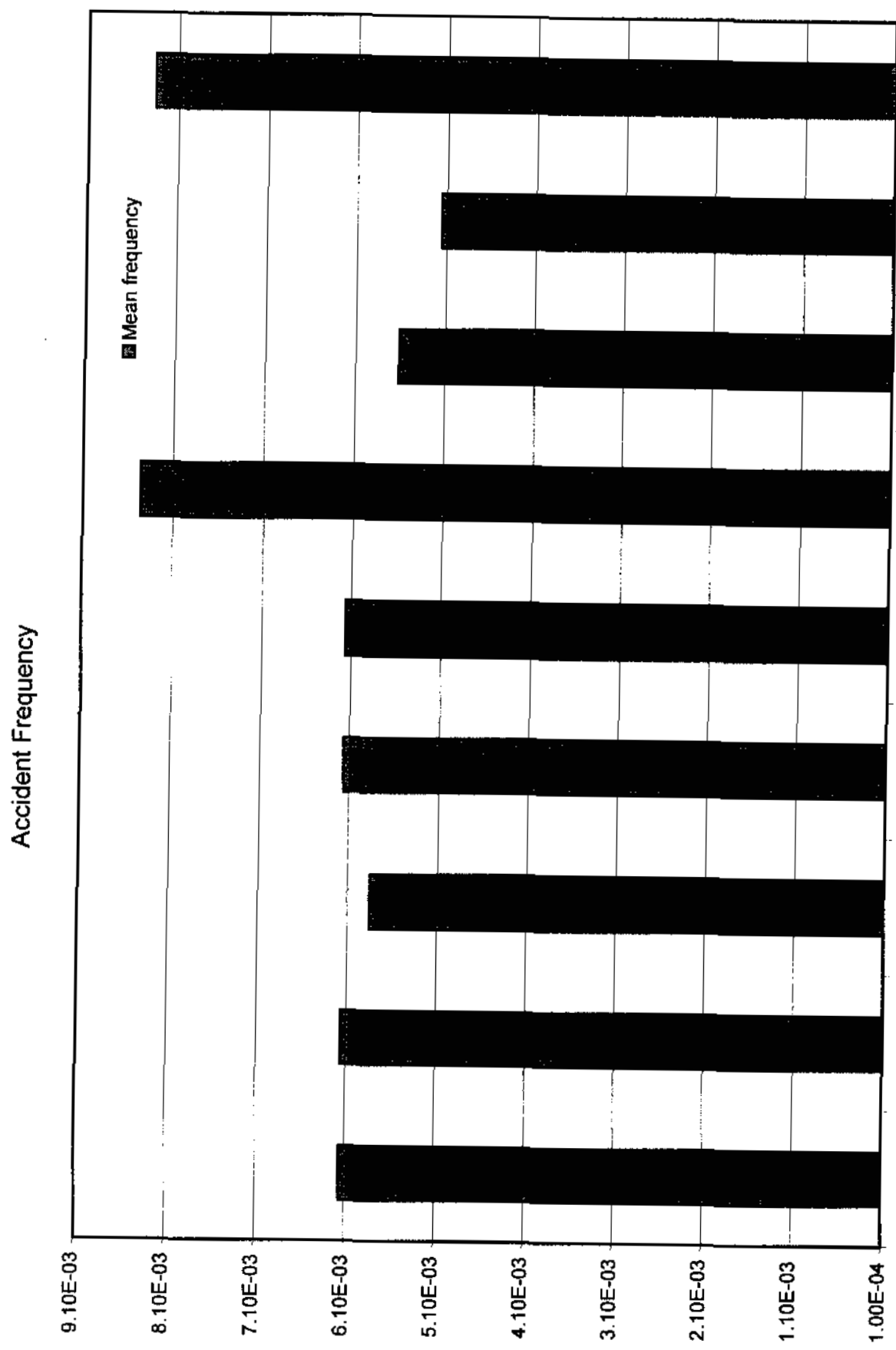

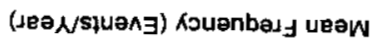

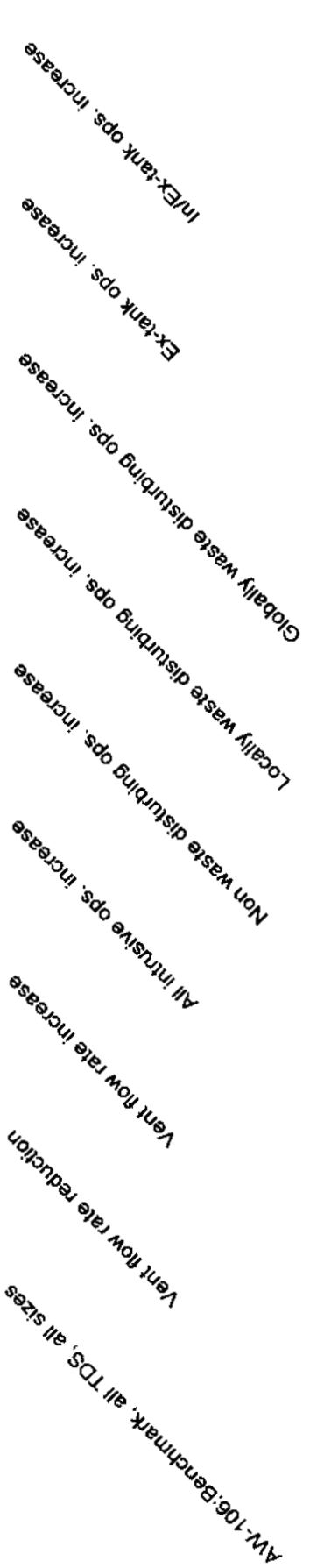


RPP-6888, Rev. 0

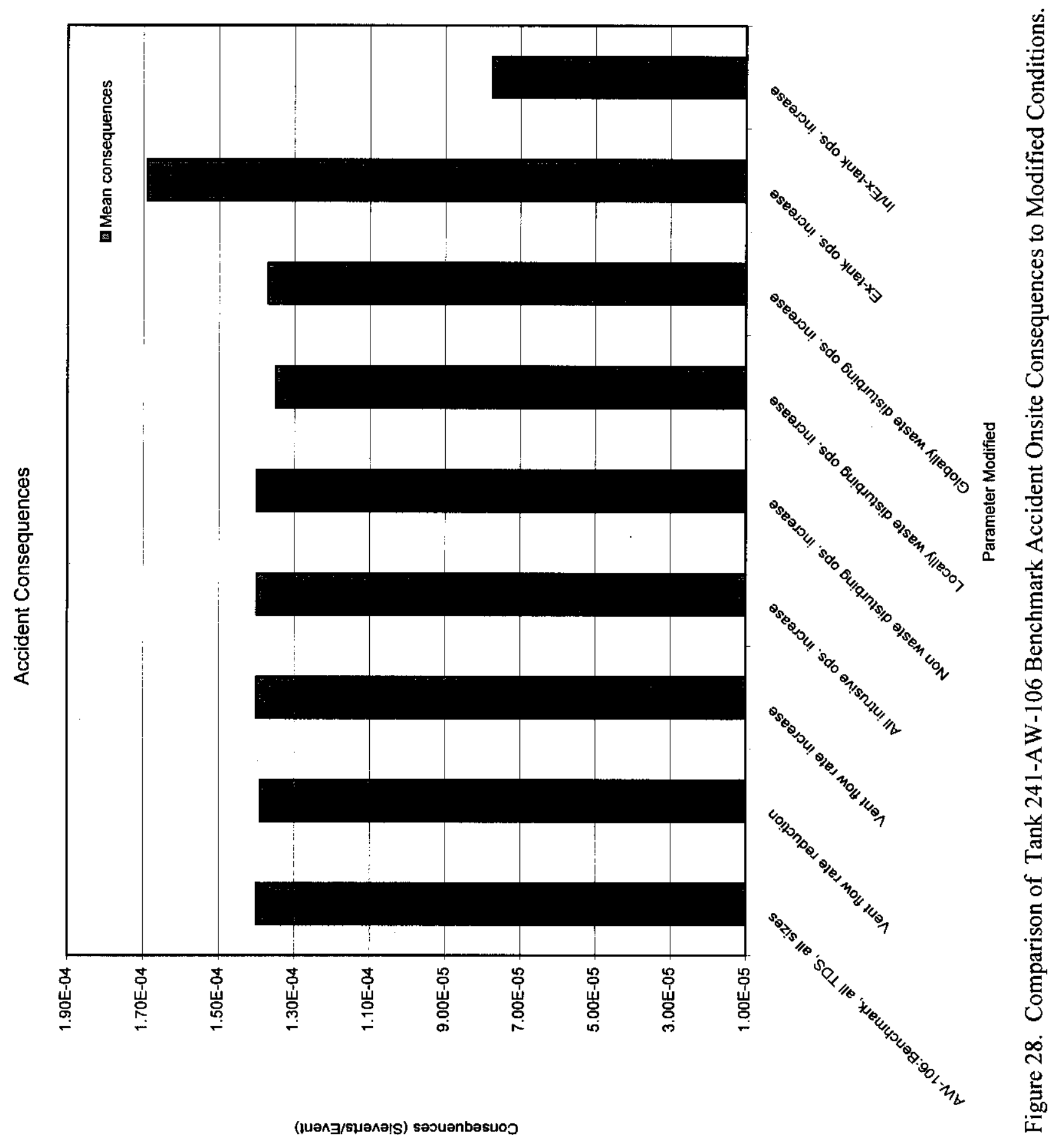


RPP-6888, Rev. 0

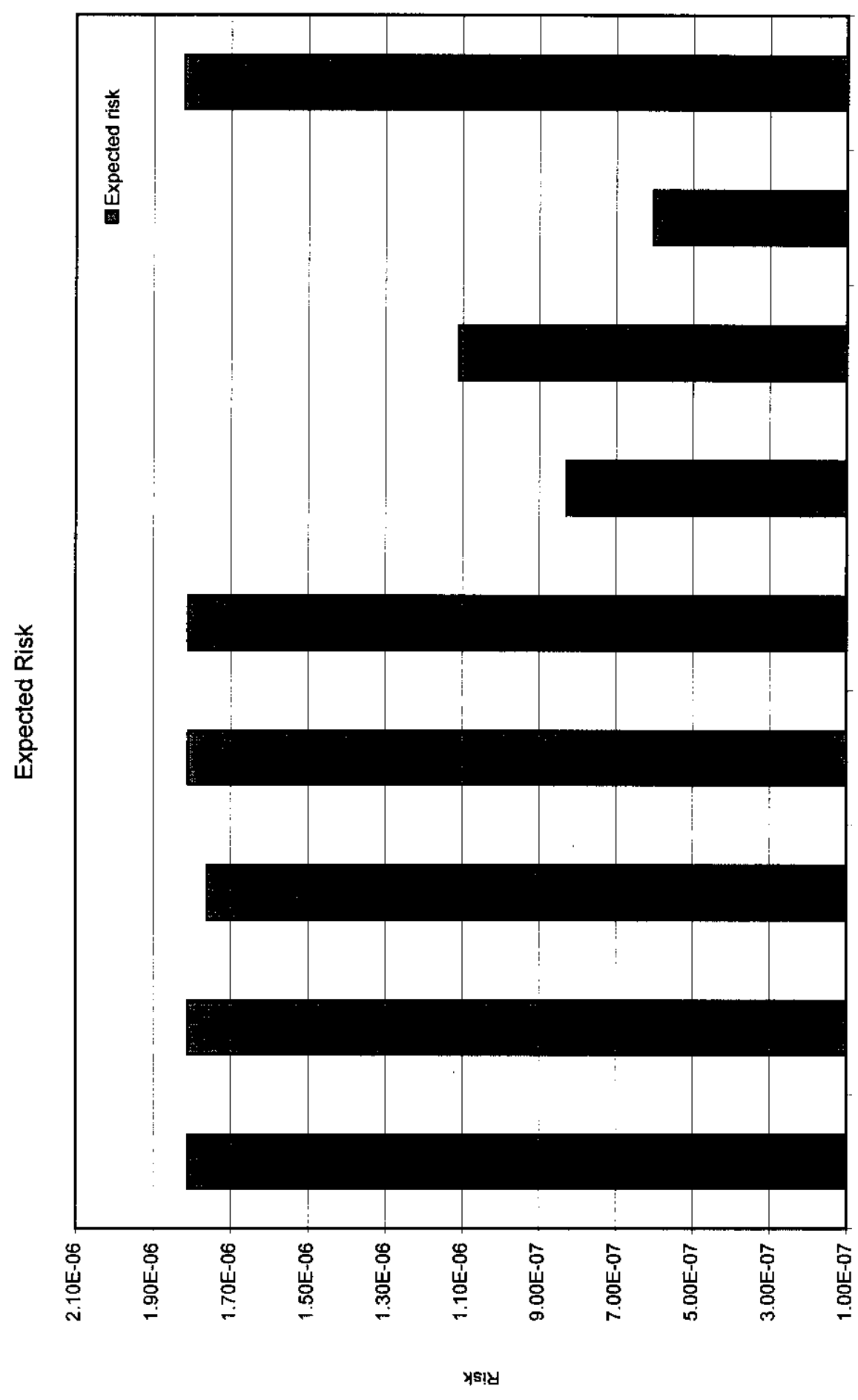

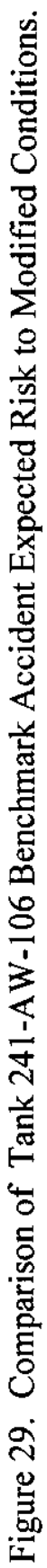


RPP-6888, Rev. 0

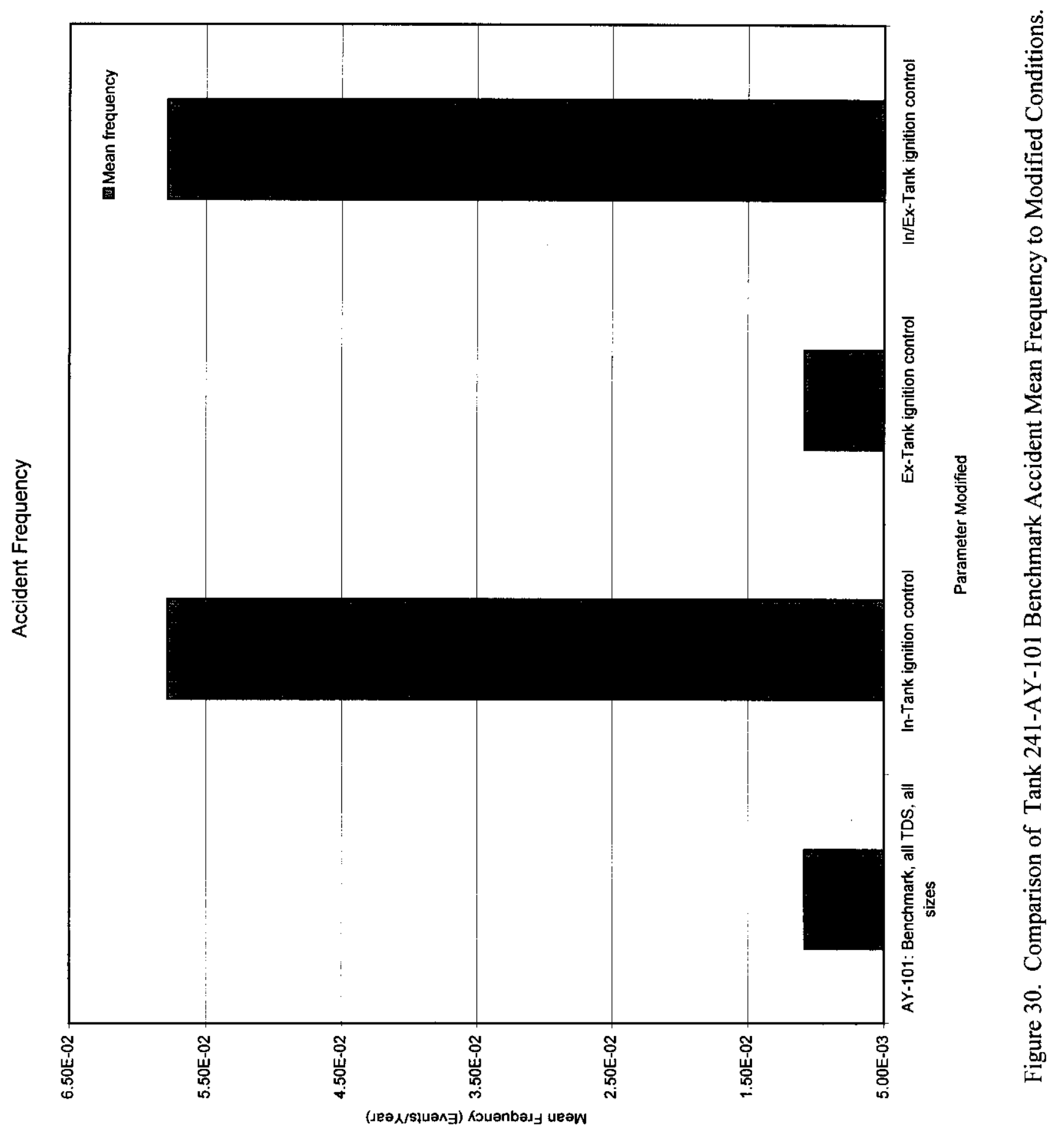

F-30 
RPP-6888, Rev. 0

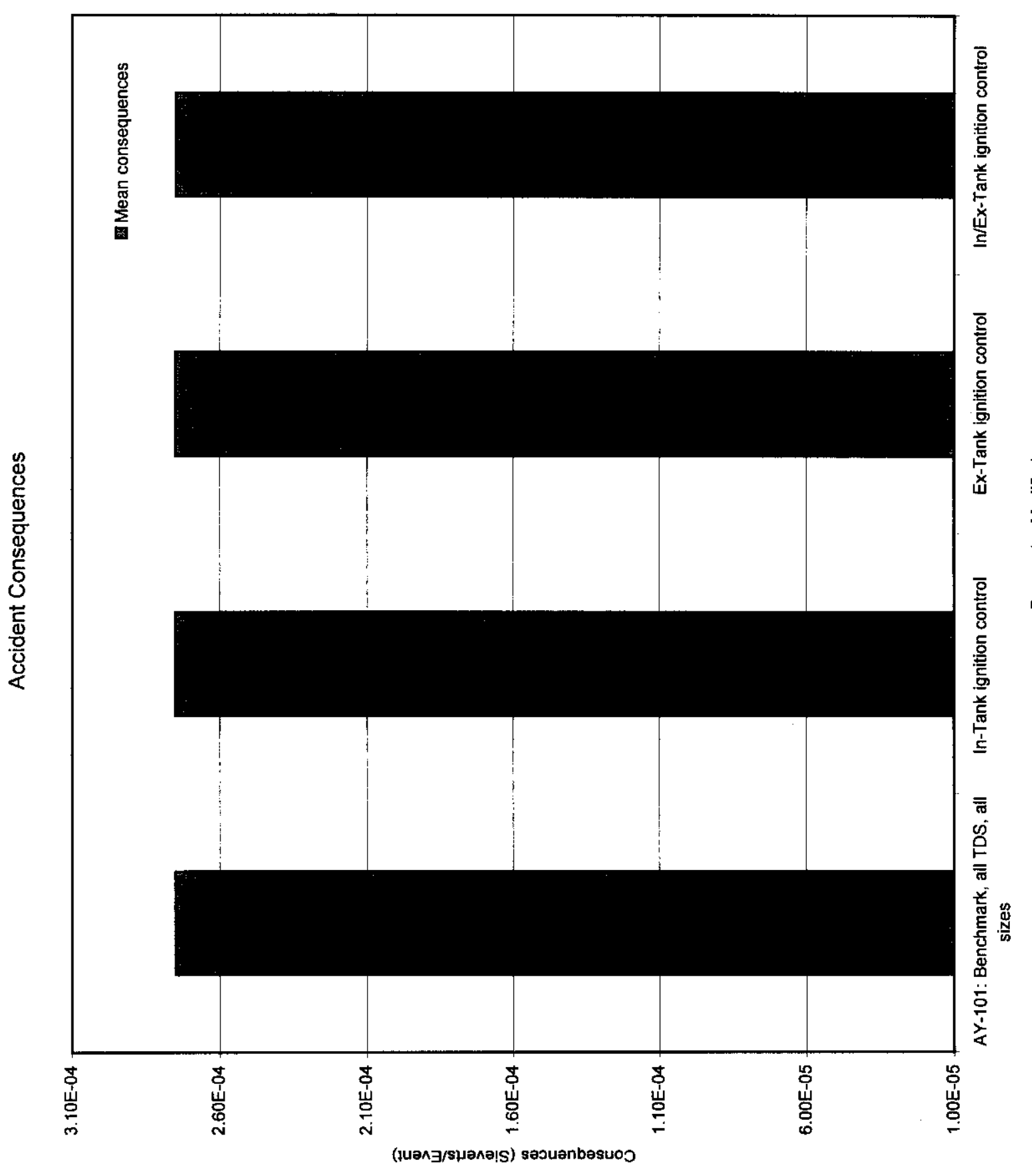

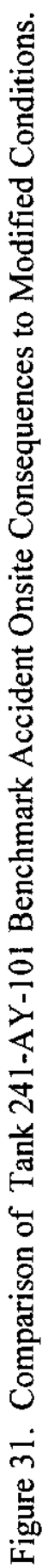

F-31 
RPP-6888, Rev. 0

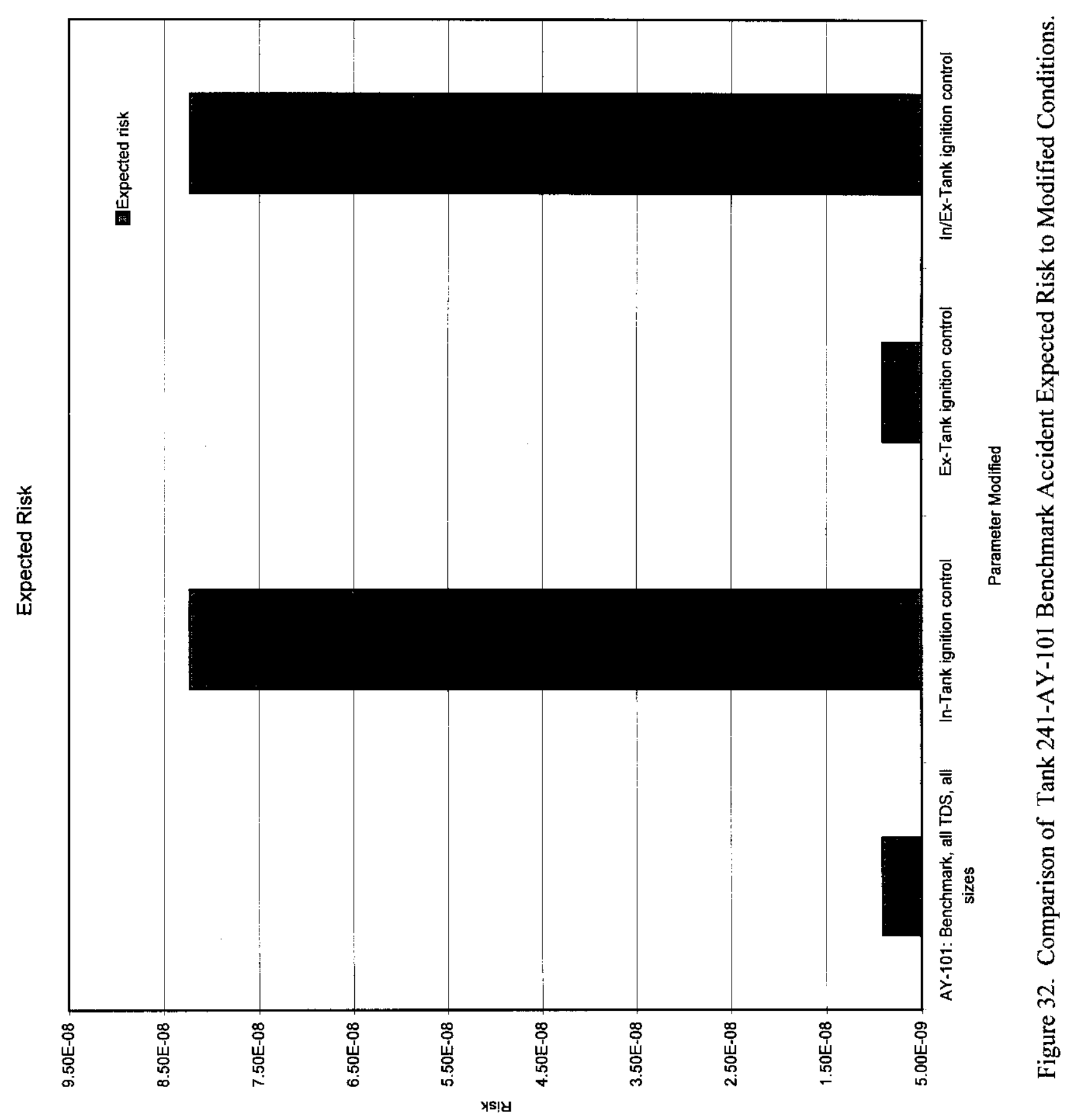

F-32 
RPP-6888 REV 0

ATTACHMENT 1

RESOLVE VERSION 2.13

ACCEPTANCE TESTING: TEST PLAN AND TEST RESULTS 
RPP-6888 REV 0

This page intentionally left blank. 


\section{TABLE OF CONTENTS}

1.0 INTRODUCTION

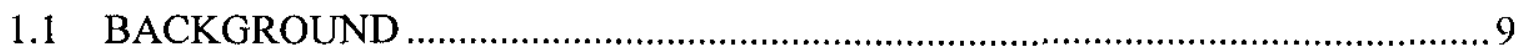

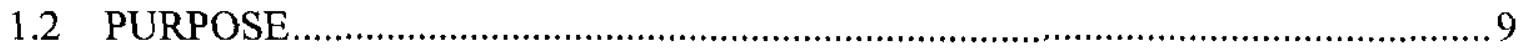

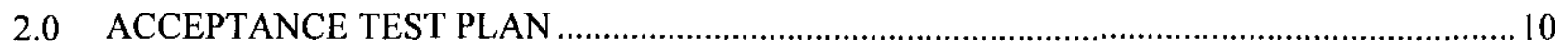

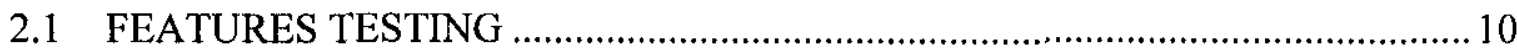

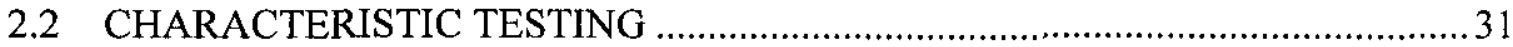

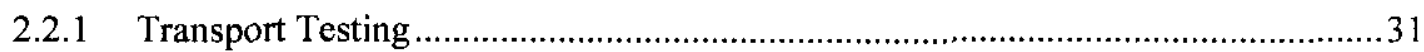

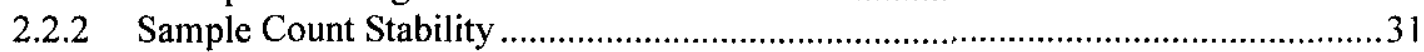

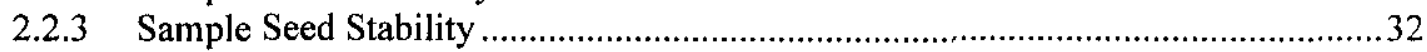

2.2.4 Sample Count and Sample Seed Stability ............................................................32

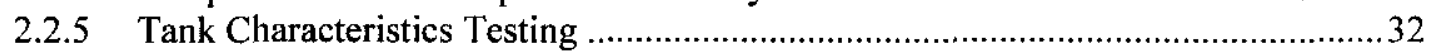

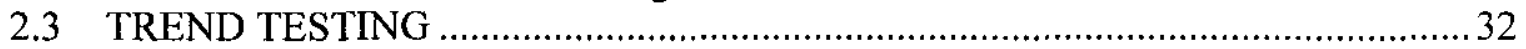

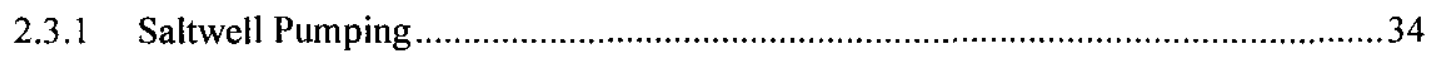

2.3.2 Waste Volume..............................................................................................

2.3.3 Number of Intrusive Operations …………………….........................................

2.3.4 Ignition Control Set ........................................................................................

2.3.5 Ventilation Rate ..........................................................................................35

2.3.6 Inerting the Tank Headspace, with Nitrogen ...........................................................35

2.3.7 Tank Failure (Cracking) Pressure ………………………………………………....36

2.3.8 Tank Failure (Collapse) Pressure ............................................................................36

2.3.9 Waste Intrusive Equipment.............................................................................36

2.3.10 Increase in Bulk Density Ratio of Solids to Liquids..............................................36

2.3.11 Increase in Waste Gas Generation Rate...............................................................37

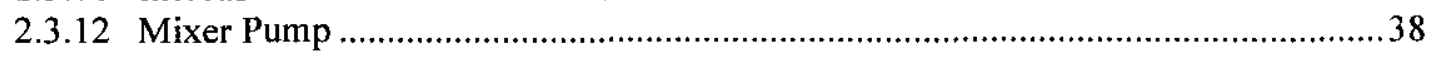

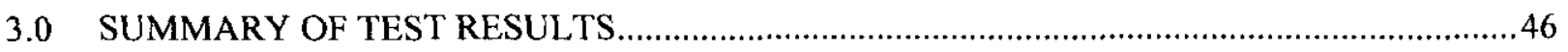

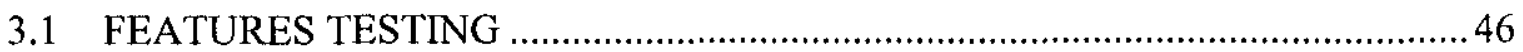

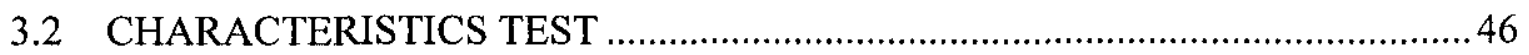

3.3 SUMMARY OF TREND TESTING RESULTS …….........................................

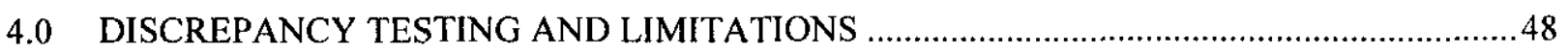

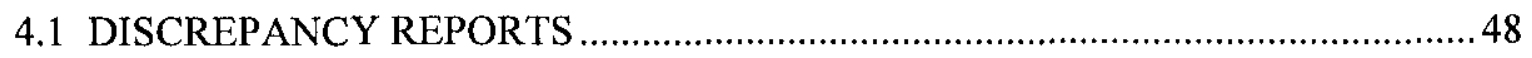

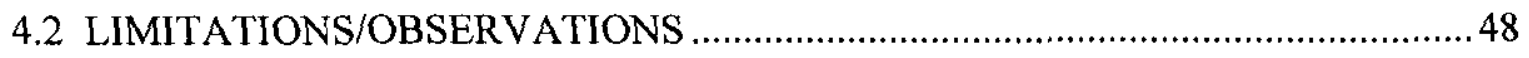

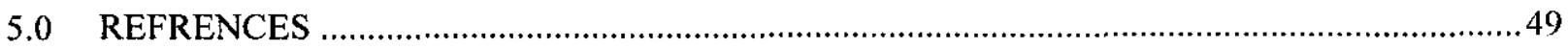


RPP-6888 REV 0

This page intentionally left blank. 


\section{RPP-6888 REV 0}

\section{LIST OF TABLES}

Table 1. Single-Shell Tanks: Acceptance Tests and Input Variables and Parameters........9

Table 2. Double-Shell Tanks: Acceptance Tests and Input Variables and Parameters .........14

Table 3. Summary of Single Shell Tanks Used in Trend Testing......................................27

Table 4. Summary of Double Shell Tanks Used in Trend Testing ....................................27

Table 5. Single-Shell Tanks: Expected Results from AcceptanceTesting....................39

Table 6. Double-Shell Tanks: Expected Results from Acceptance Testing.................43

Table 7. Summary of the Results of the Characteristics Tests Performed............................49 
RPP-6888 REV 0

This page intentionally left blank. 


\section{RPP-6888 REV 0}

\section{LIST OF FIGURES}

Figure 1. DST: Percent Increase in Total GRE Deflagrations and Detonations due to Parameter Modification.

Figure 2. SST: Percent Increase in Total GRE Deflagrations and Detonations due to Parameter Modifications

Figure 3. DST: Change in Accident Frequency due to Parameter Modification. F-13

Figure 4. SST: Change in Accident Frequency due to Parameter Modification F-14

Figure 5. DST: Change in Accident Consequences due to Parameter Modification. F-15

Figure 6. SST: Change in Accident Consequences due to Parameter Modification F-16

Figure 7. DST: Change in Expected Risk due to Parameter Modification. F-17

Figure 8. SST: Change in Expected Risk due to Parameter Modification F-18

Figure 9. DST: Percent Increase in GRE Behavior due to Operations and Engineering Change in Waste Intrusive Equipment Parameters

Figure 10. SST: Percent Increase in GRE Behavior due to Operations and Engineering Changes in Waste Intrusive Equipment Parameters F-20

Figure 11. .DST: Changes in GRE Behavior due to Changes in Waste Characteristics 


\section{RPP-6888 REV 0}

This page intentionally left blank. 


\section{RPP-6888 REV 0}

\subsection{INTRODUCTION}

\subsection{BACKGROUND}

To assist in quantifying the risk of the Flammable Gas Safety Issue, an accident Analysis Tool has been developed using commercially available software and tank characterization data. In addition to using available data and analysis results, the process also uses formal systematic expert elicitation on flammable gas technical parameters for which no data exists. The Analysis Tool is a computer code called Resolve!. The Analysis Tool is designed to quantify the risk and uncertainty of combustion accidents in actual or representative tanks and the change in risk that would result from using different control strategies. Resolve! is used to help identify optimal controls for each tank and analyze the implementation of the controls prior to actual implementation in the field. This supports the actions needed to update the Authorization Basis.

The acceptance and validation testing of the precursor version, RESOLVE! Version 1.51 , is documented in RESOLVE Version 1.51 Acceptance Testing: Test Plan and Test Results. The major improvement of the refined safety analysis tool is the ability to analysis and evaluate Double-Shell Tanks (DSTs). Additional changes to the refined safety analysis tool include, buoyant displacement model, revised mass balance, waste intrusive equipment burns, mixer pump, waste transfer and the graphical user interface (GUI). The revised version is identified as Resolve! Version 2.13. This Attachment documents the acceptance testing performed on Version 2.13.

\subsection{PURPOSE}

The acceptance test is the primary method used by the Tank Farm Contractor (TFC) Team to accept the Analysis Tool, Resolve! Version 2.13. This version of the Analysis Tool (Resolve! Version 2.13) is not intended to be used to identify, analyze, and select safety related controls; therefore, the acceptance test and results are documented in this Attachment 1. This testing has been performed to enhance the TFC Team understanding of the Analysis Framework and implication of the Analysis Tool results.

This Attachment 1 provides the results of acceptance testing of Resolve, Version 2.13. The results of acceptance testing are used to do the following:

Confirm that the results of Resolve! Version 2.13 , trends logically

- $\quad$ Ensure that Resolve! Version 2.13, performs within the predefined parameters

- Determine the status of previously identified Analysis Tool user interface issues and calculational errors.

- Identify any deficiencies and limitations for application of Version 2.13

Whenever an area of acceptance testing is unsuccessful, two approaches are used to evaluate the condition. Unless it is evident that an error (e.g., computational or GUI) exists, the code developers and the TFC Team convene to determine if the suspected error is correct (i.e., whether the tank behaved as expected based on the parameter modified and the correct calculational formulae) or whether a deficiency exists (i.e., results are unexpected based on parameter modification and the calculational formulae). If the error is obvious or a deficiency was noted, a discrepancy report is submitted. The 


\section{RPP-6888 REV 0}

code developers use the discrepancy report to correct and improve the functionality of the refined safety analysis tool. Section 2.0 identifies the tests performed, Section 3.0 summarizes testing results, Section 4.0, lists references and Appendix A provides a listing of Discrepancy Reports and report status, and Appendices $\mathrm{B}$ and $\mathrm{C}$ provide detailed trend test analysis results.

\subsection{ACCEPTANCE TEST PLAN}

RESOLVE! Version 1.51 was improved to include the analysis and evaluation of Double-Shell Tanks (DSTs). Additional changes to the refined safety Analysis Tool include the buoyant displacement model, the revised mass balance, waste intrusive equipment burns, the mixer pump, waste transfer and the graphical user interface (GUI). The impacts associated with these changes have been previously determined and are documented (Slekak et al. 1999, Slezak and Bratzel 1997). The modified version is identified as Resolve! Version 2.13.

The acceptance test plan (ATP) is the primary method used by the TFC Team to determine acceptance of Resolve! Version 2.13. This plan will also verify the closure of discrepancy or deficiency reports generated during the testing and implementation of Version 2.13. Two acceptance test plans have been developed, one addresses the Single-Shell Tanks (SSTs) and the other addresses the DSTs. To provide a better understanding of the behavior of the waste the two test plans evaluate the same parameter changes; however, where appropriate additional tests have been identified based on the characteristics of the waste contained in the tanks. For example, buoyant displacement gaseous release events are only evaluated with DSTs. Tables 1 and 2 identify the tests and input parameters and variables modified for each test that will be performed for SSTs and DSTs, respectively.

Each ATP is divided into three primary areas. The three areas are: "Features Testing," "Characteristics Testing," and "Trend Testing." The purpose of the "Features Testing" (see Section 2.1) is to ensure that all of the options and features of the computer code run. The purpose of the "Characteristics Testing" (see Section 2.2) is to verify that the calculated results are consistent with each other and repeatable and to quantify the baseline stability or behavior of the computer code. "Trend Testing" (see Section 2.3) is performed to evaluate the effects of the changes to the parameter values on the frequency and consequence trends (i.e. increase, decrease, or no change) associated with gas deflagrations or detonations. In addition, previously generated discrepancy reports (see Section 2.4) as well as existing and previously identified code limitations (see Section 2.5) are identified and, where appropriate, tested and evaluated. Each of these test areas is described further in the following.

Discrepancies and issues identified during testing are discussed and summarized in Section 4.0.

\subsection{FEATURES TESTING}

Testing the features of Resolve! 2.13 ensures that it runs properly and produces the proper outputs while various available options are selected. To test all the features, an analyst systematically works through the available screens and makes every allowable adjustment. In addition, feature testing includes verifying that the code and data libraries are consistent with the baseline documentation.

Additional features tests are identified for DSTs. The additional DST features test include turning on the mixer pump (normally off) and creating buoyant displacement GREs in non-buoyant tanks by modifying the characteristics of the waste. 


\section{RPP-6888 REV 0}

Acceptable behavior is that all features perform the function intended by the programmers and that no feature causes the program to "crash" or result in an error message. The baseline code and the data libraries must also be consistent with the documentation. This testing also includes running sample cases provided by the code developers to verify the installation of the computer code. The verification involves comparing the results of the sample case run(s) at a user workstation to the results of the code developer sample case run(s). Results of Feature Testing are summarized in Section 3.1. Any discrepancy reports generated as a result of this testing are presented in Appendix A. 


\section{RPP-6888 REV 0}

This page intentionally left blank

Att- 12 


\section{RPP-6888 REV 0}




RPP-6888 REV 0

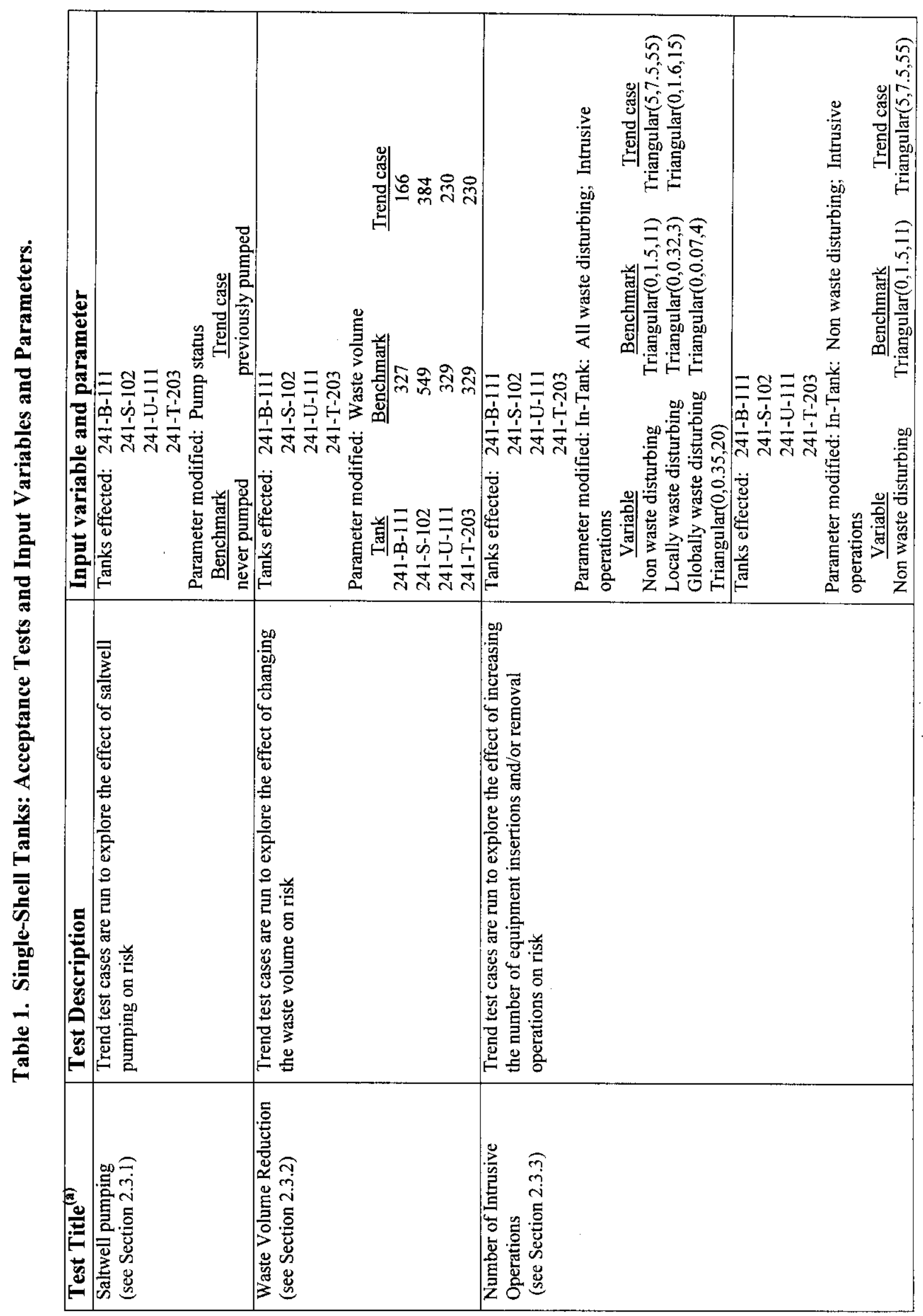




\section{RPP-6888 REV 0}

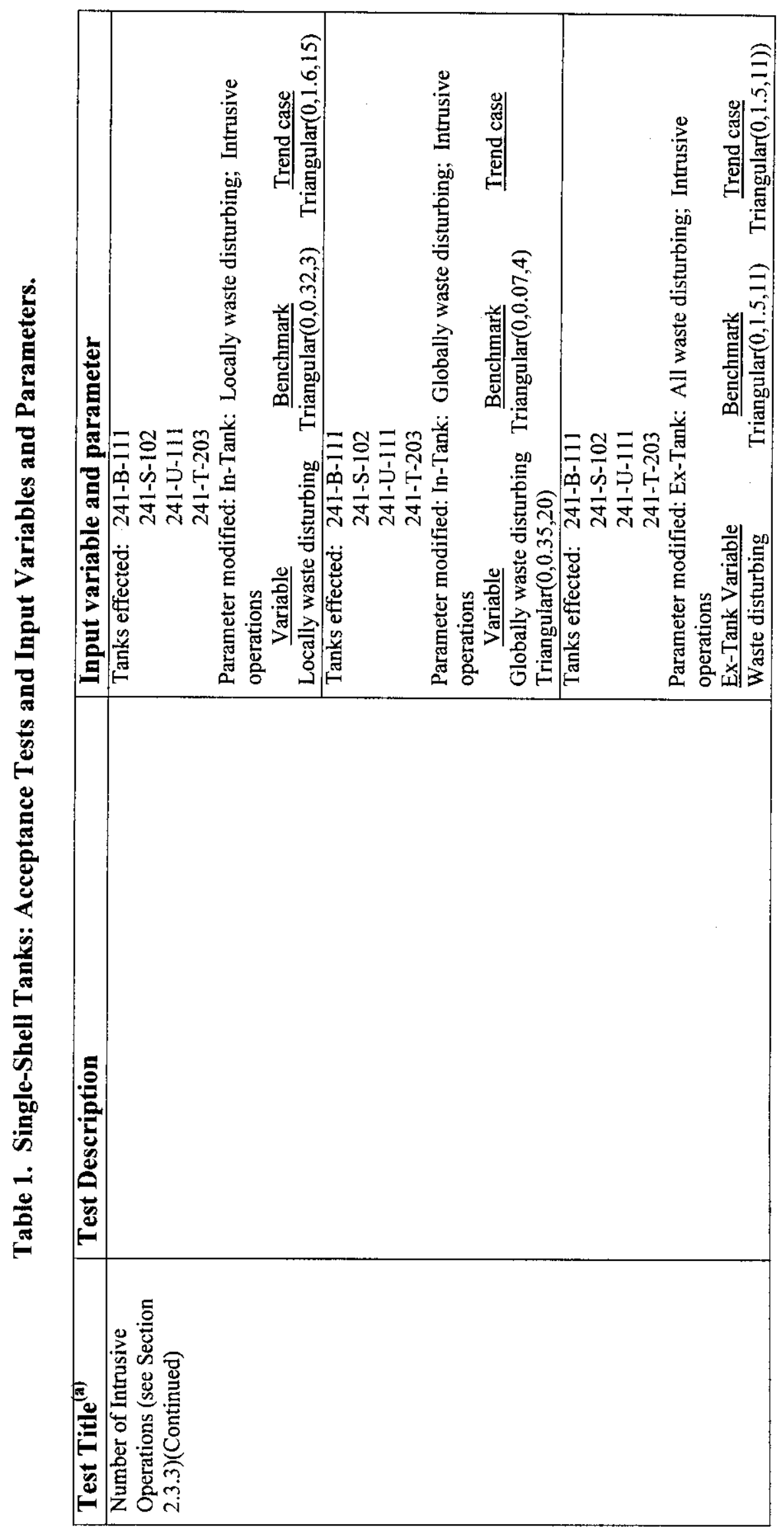

Att-15 


\section{RPP-6888 REV 0}

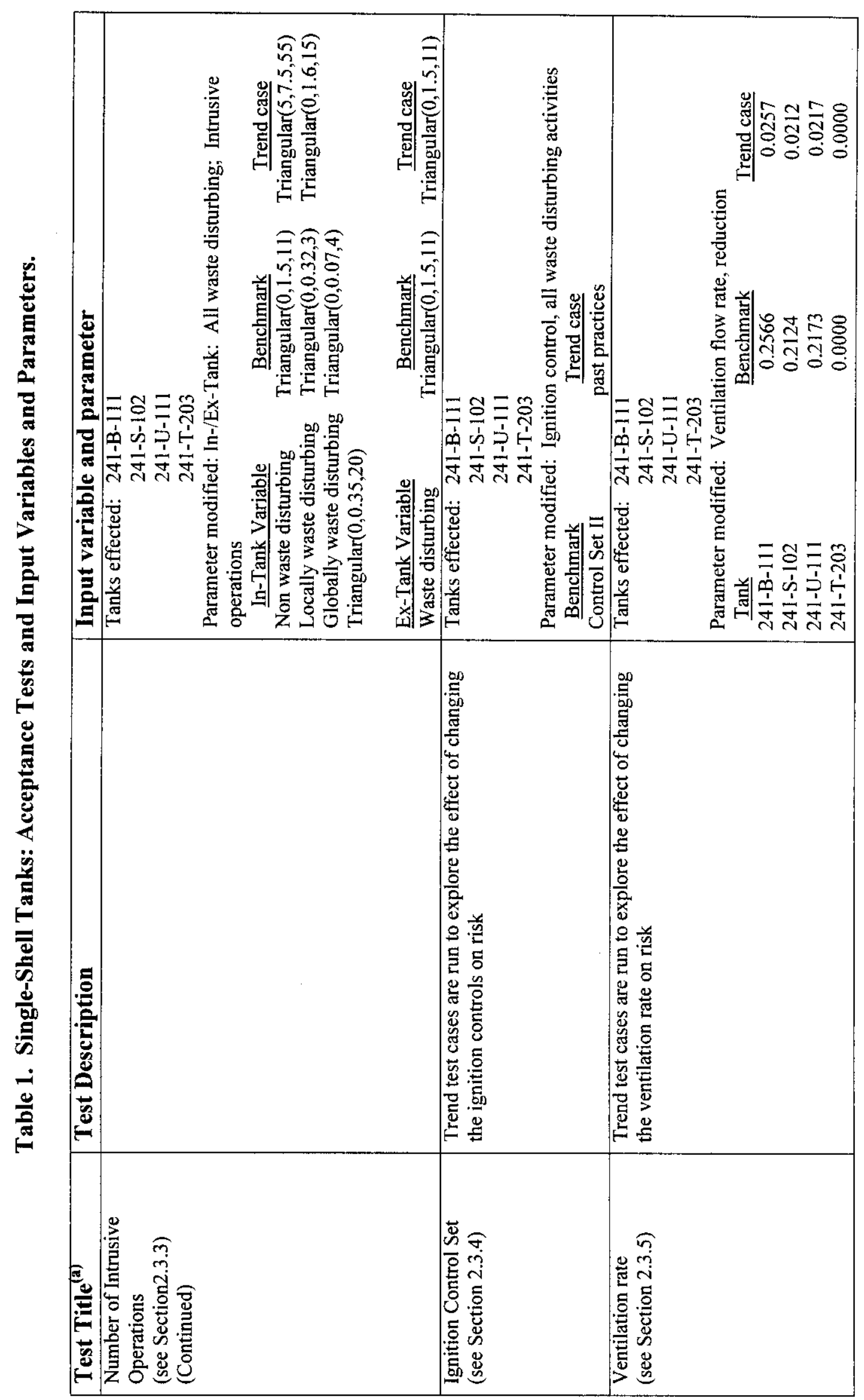


RPP-6888 REV 0

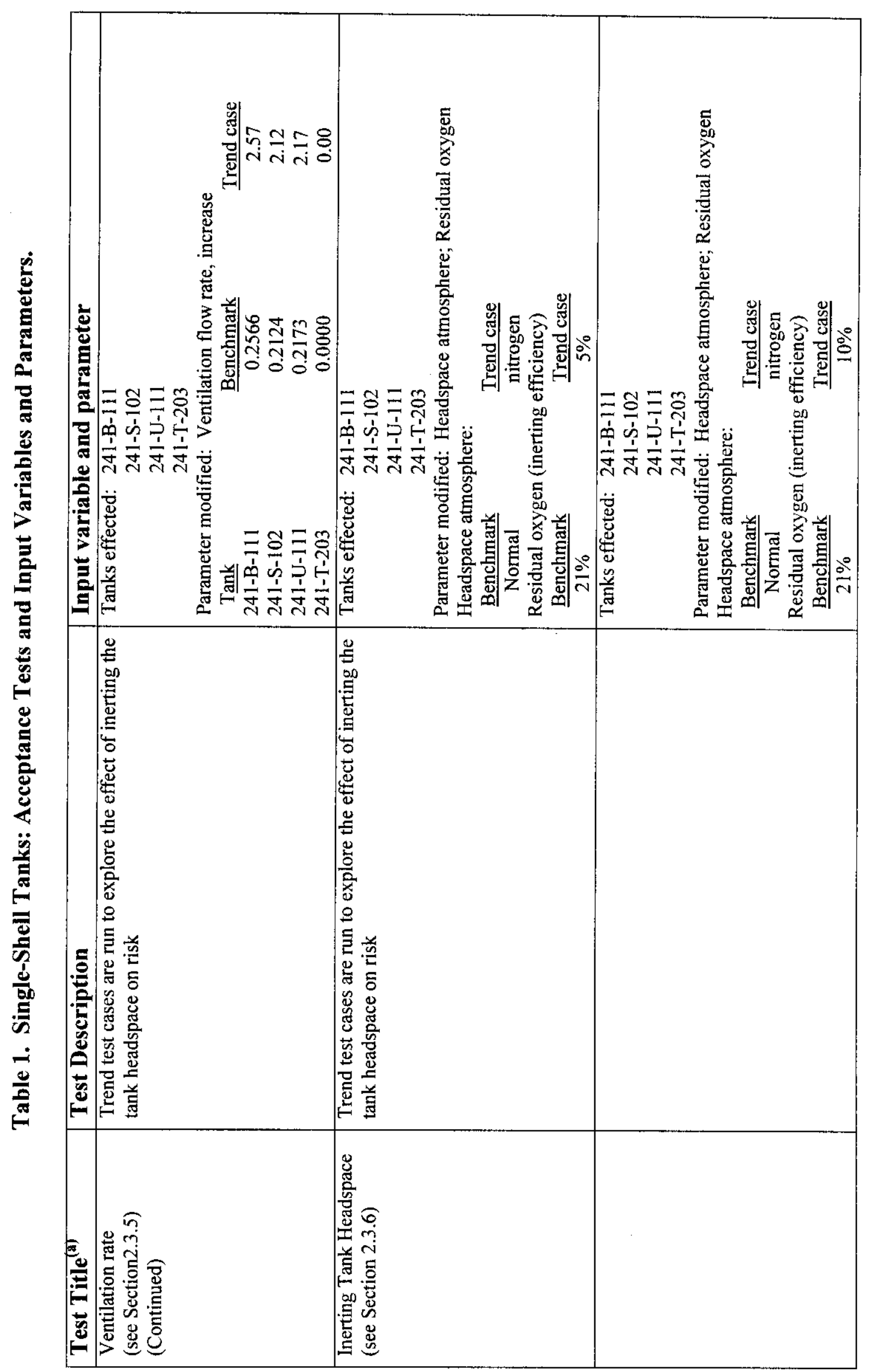

Att- 17 
RPP-6888 REV 0

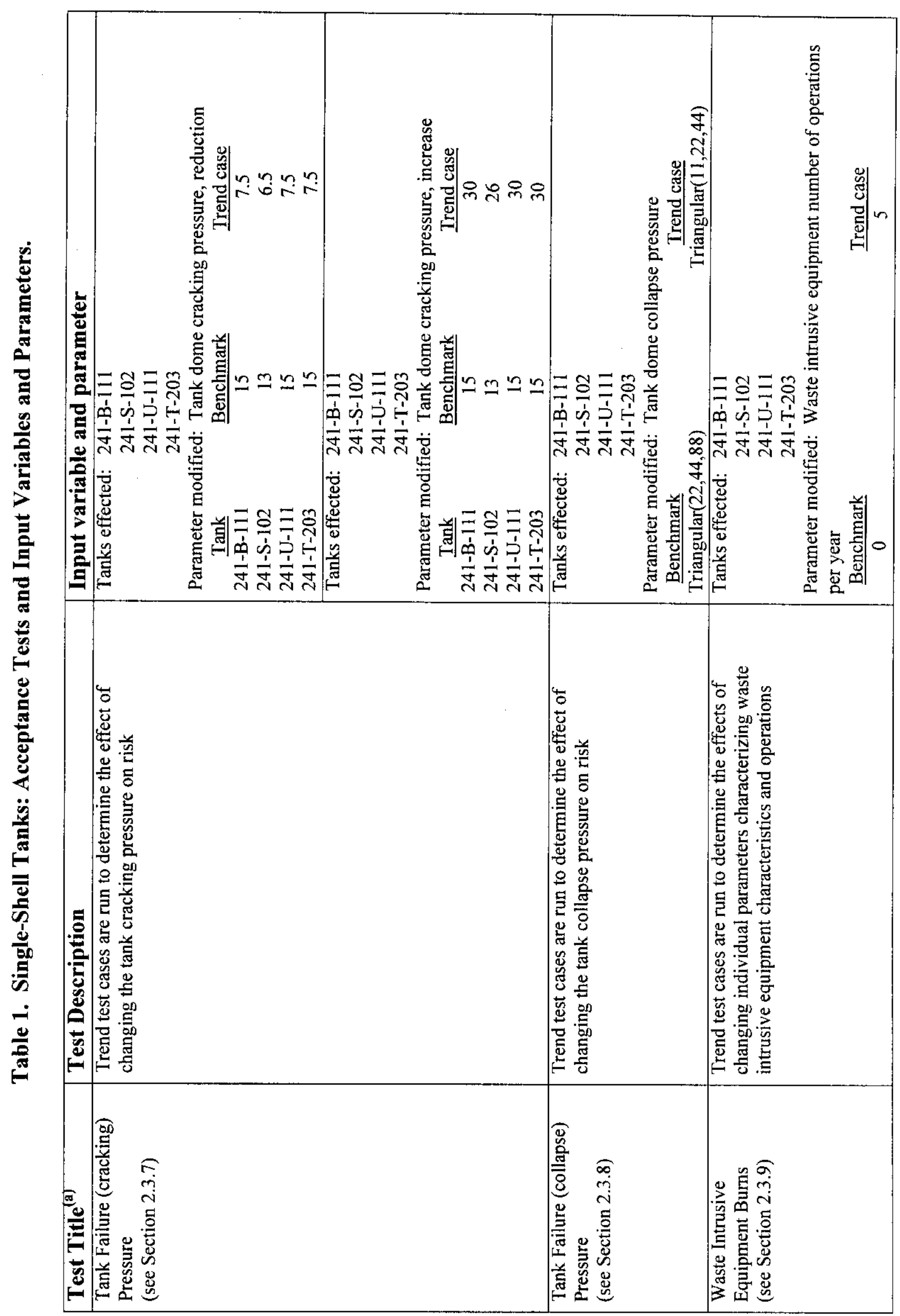


RPP-6888 REV 0

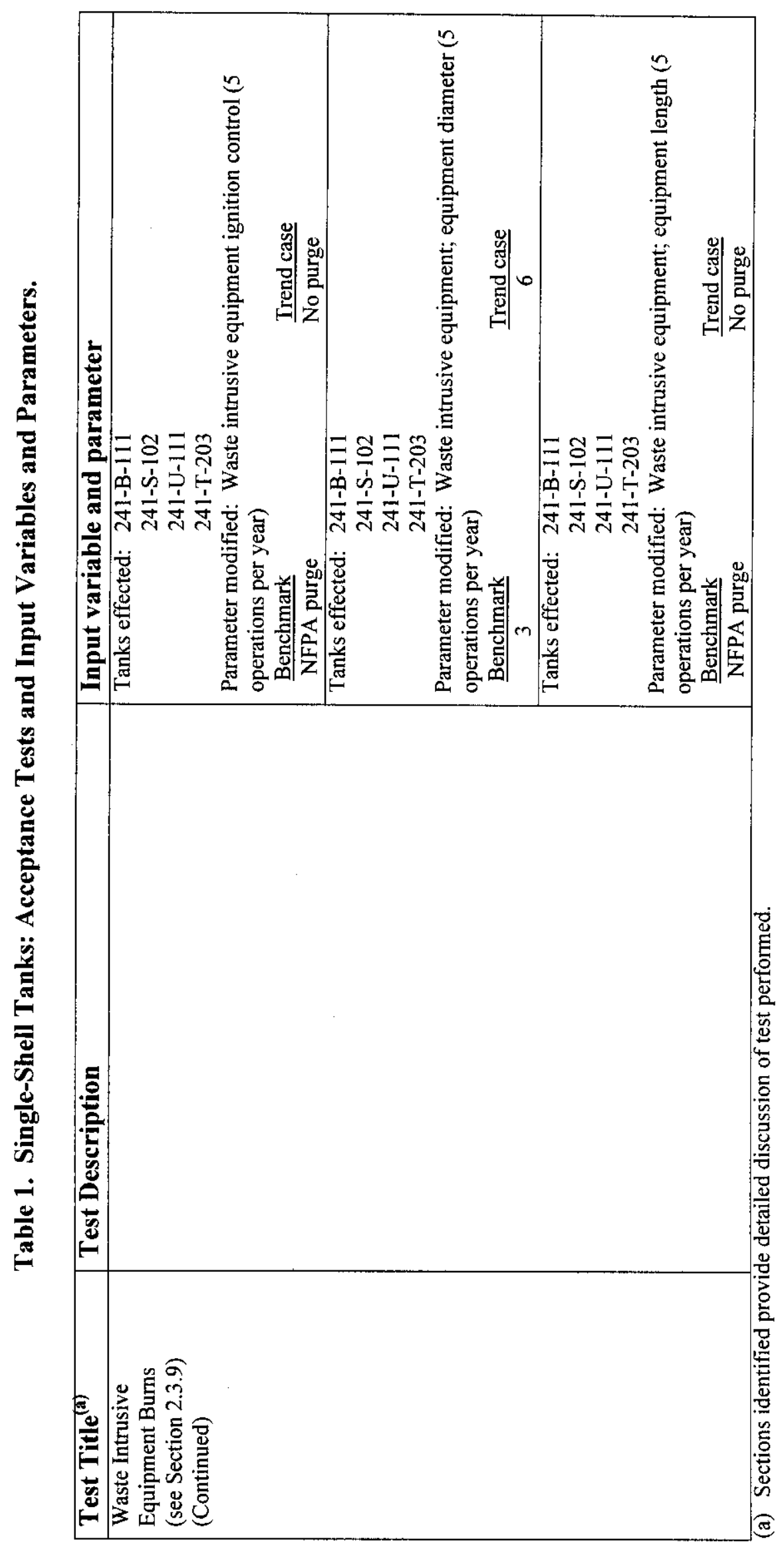

Att-19 


\section{RPP-6888 REV 0}

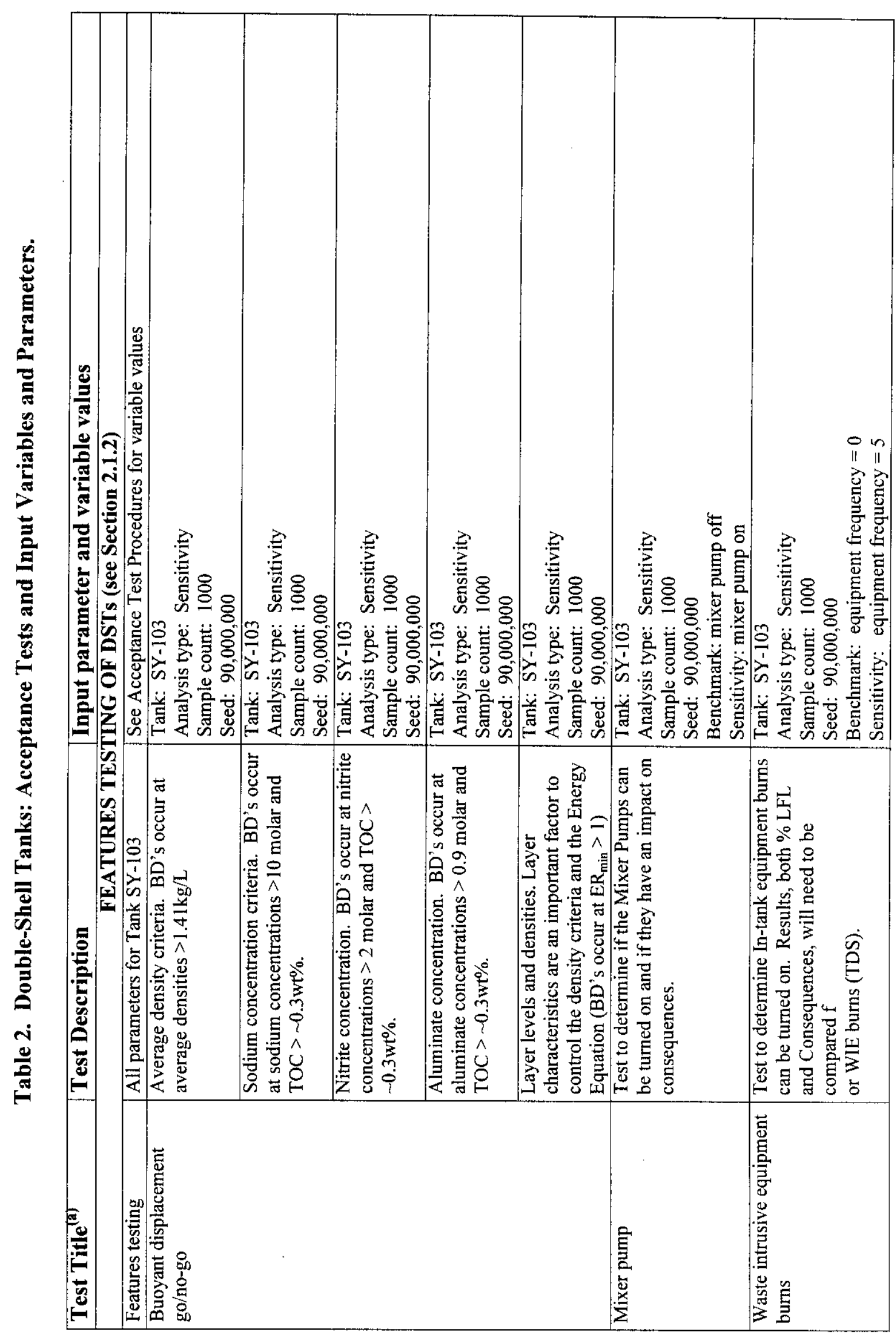




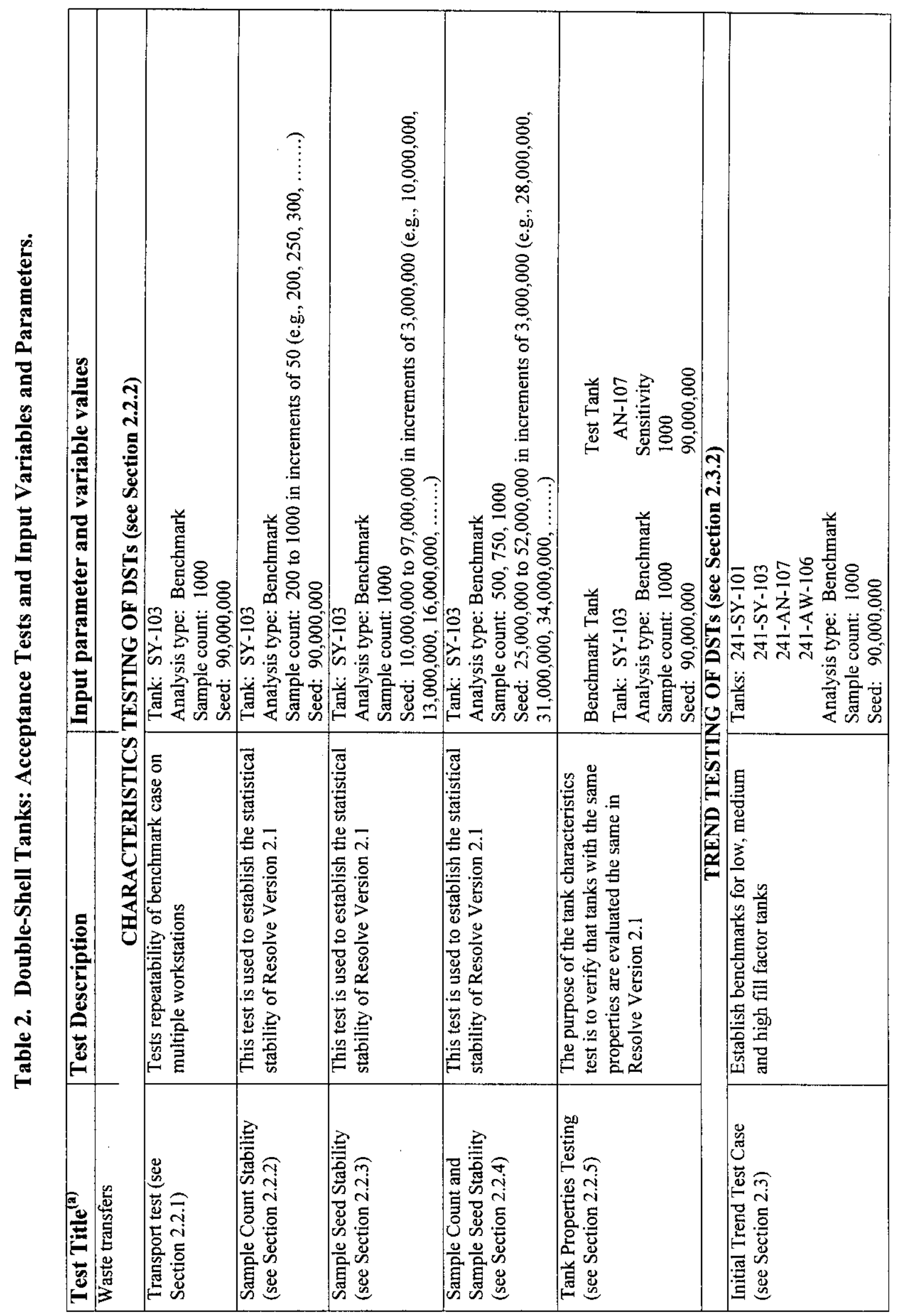


RPP-6888 REV 0

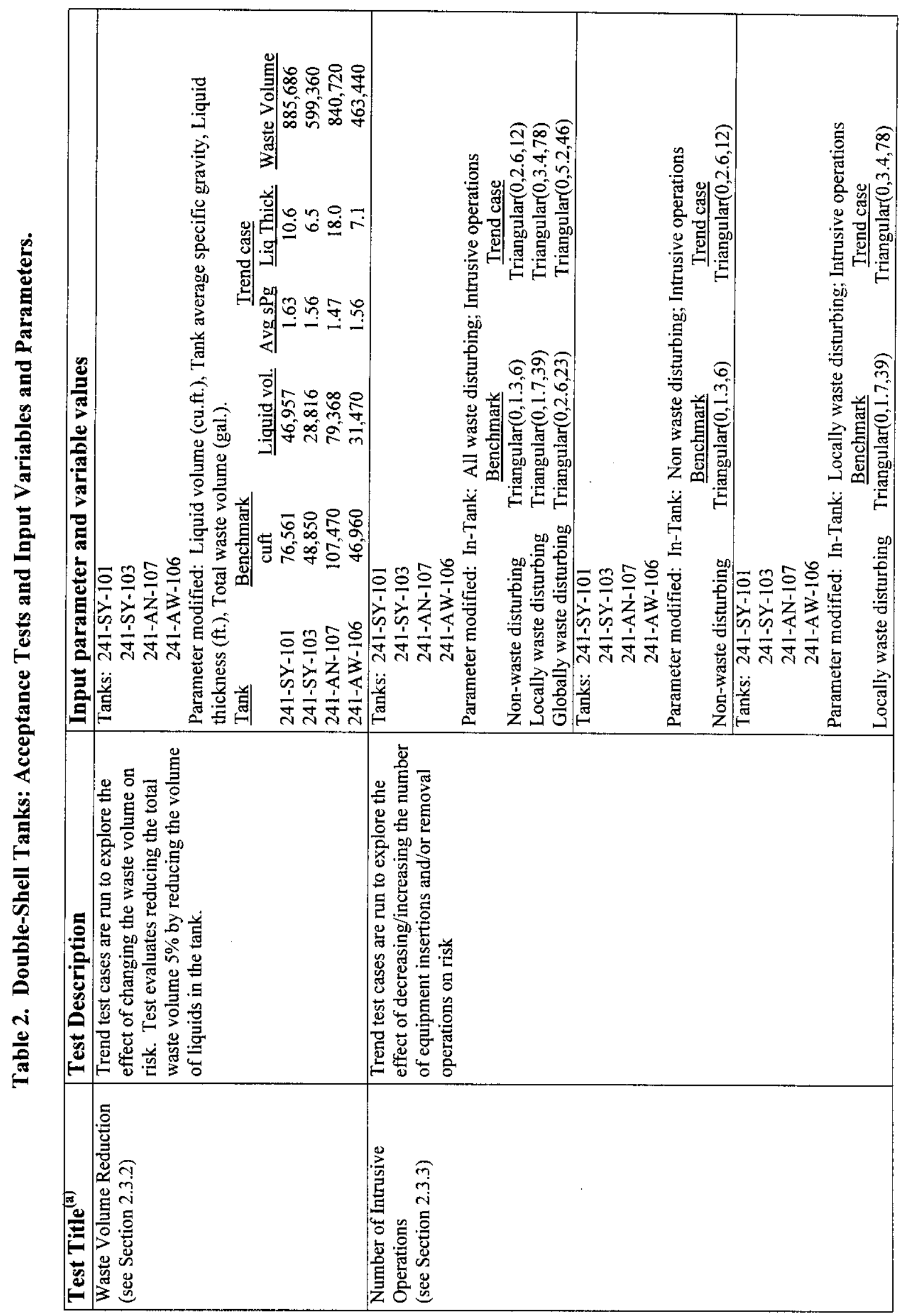




\section{RPP-6888 REV 0}

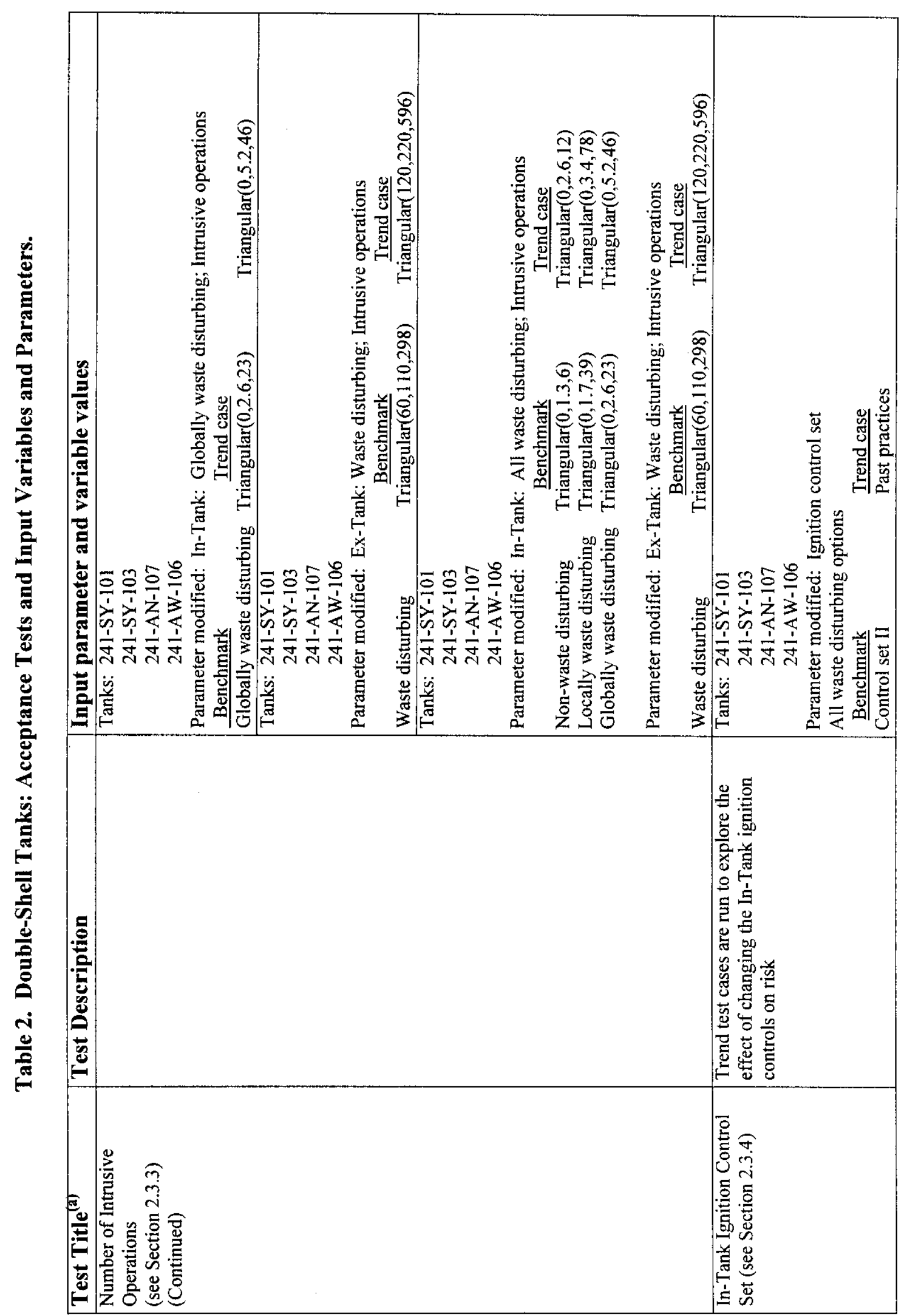


RPP-6888 REV 0

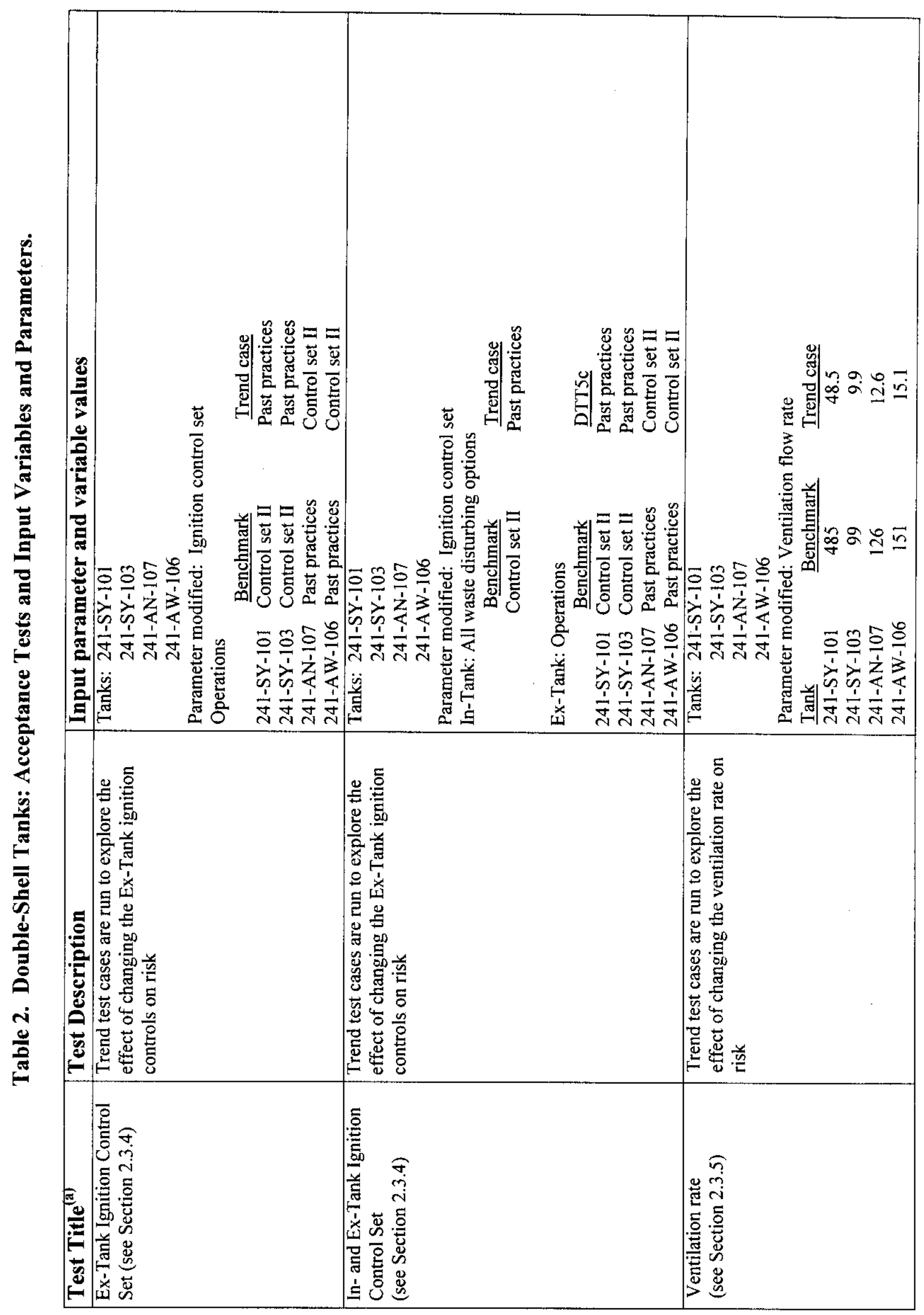


RPP-6888 REV 0

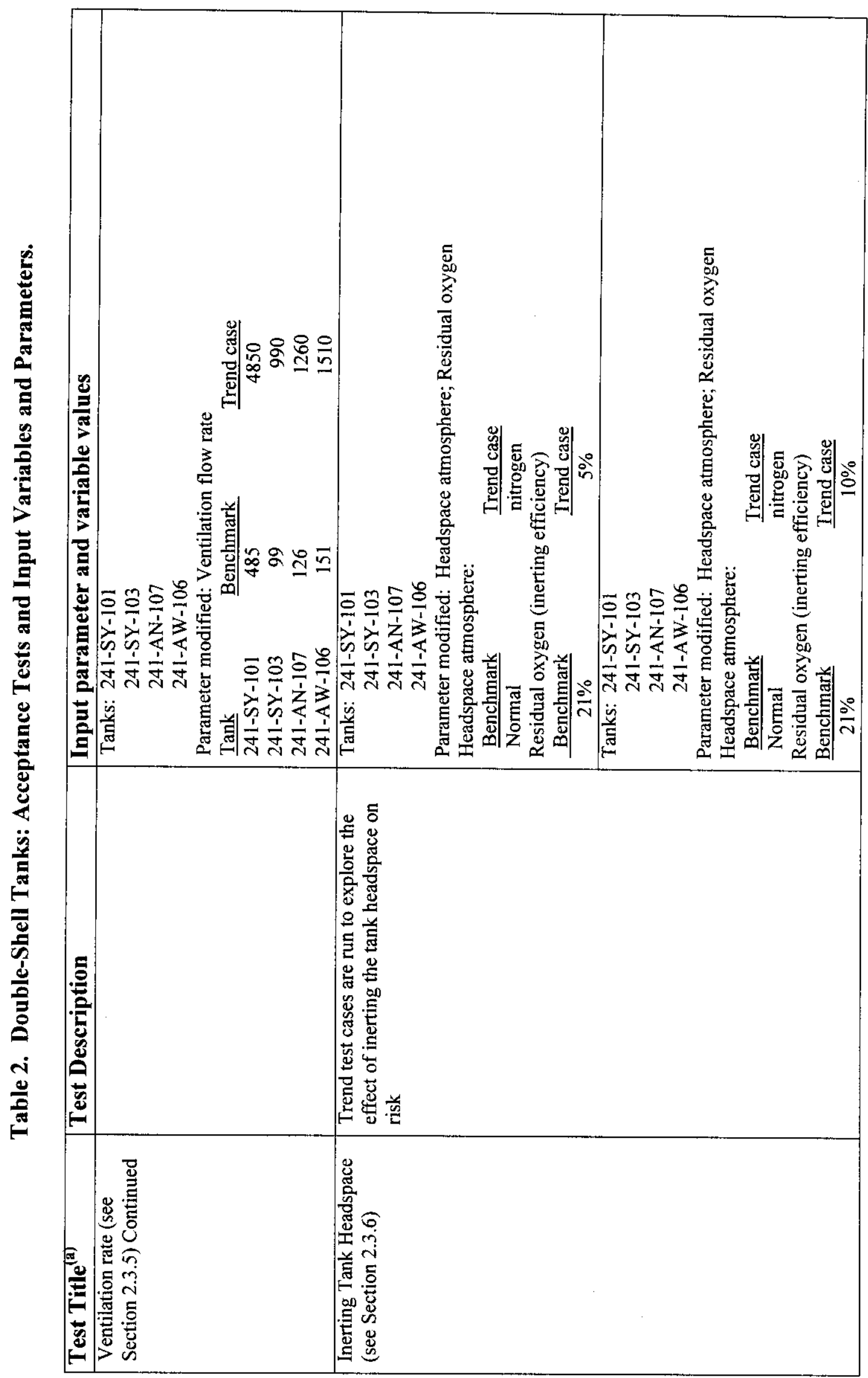

Att-25 
RPP-6888 REV 0

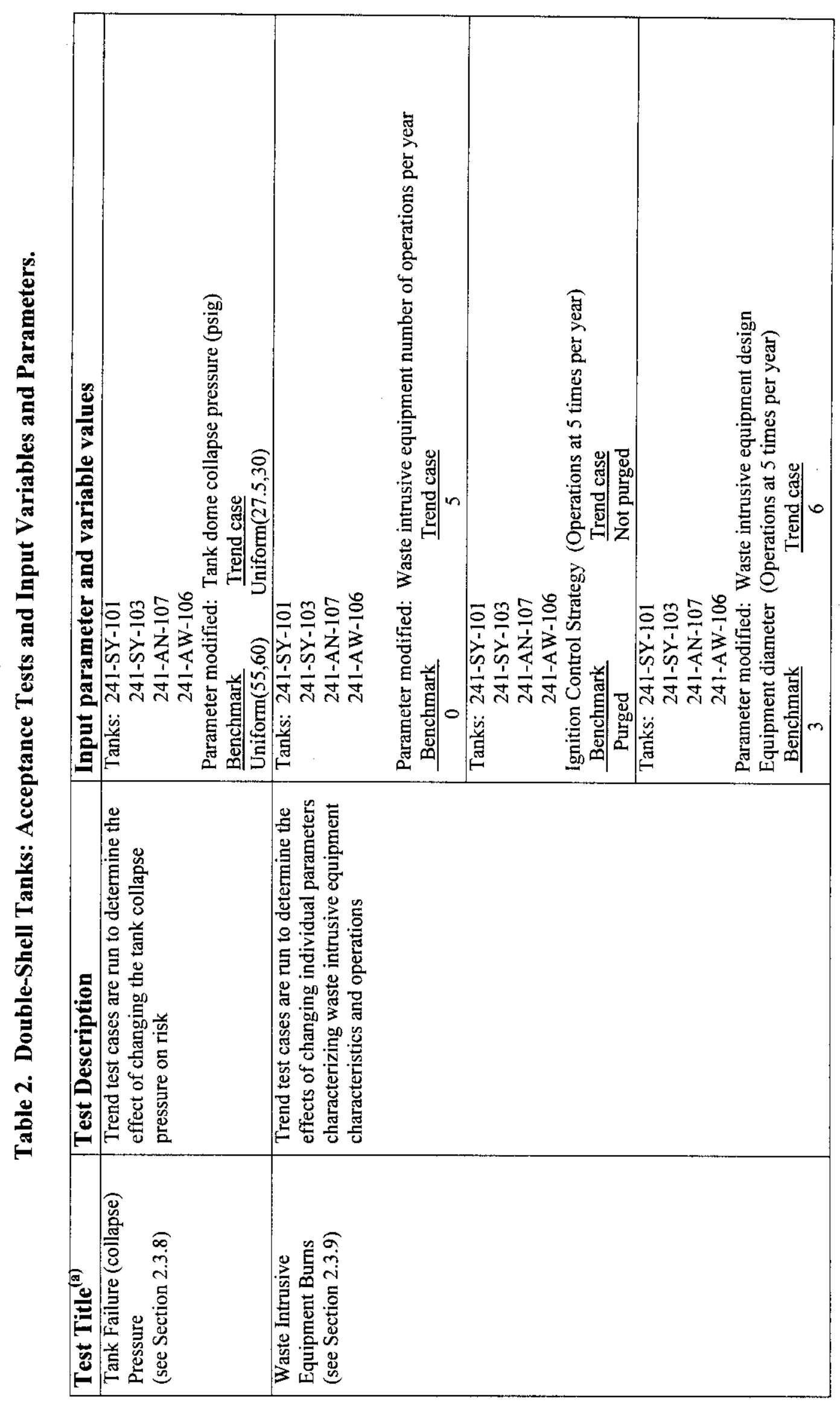




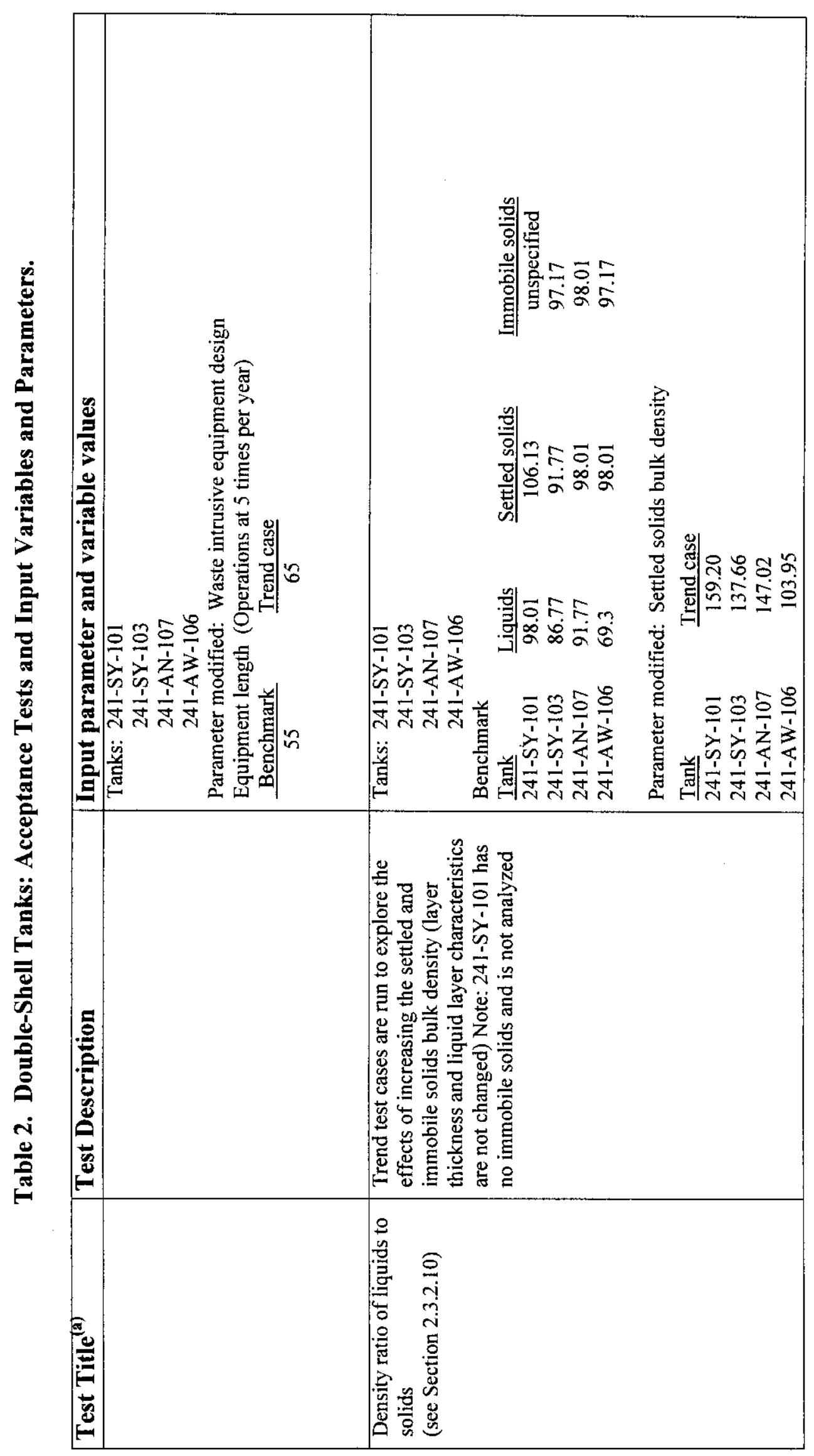




\section{RPP-6888 REV 0}

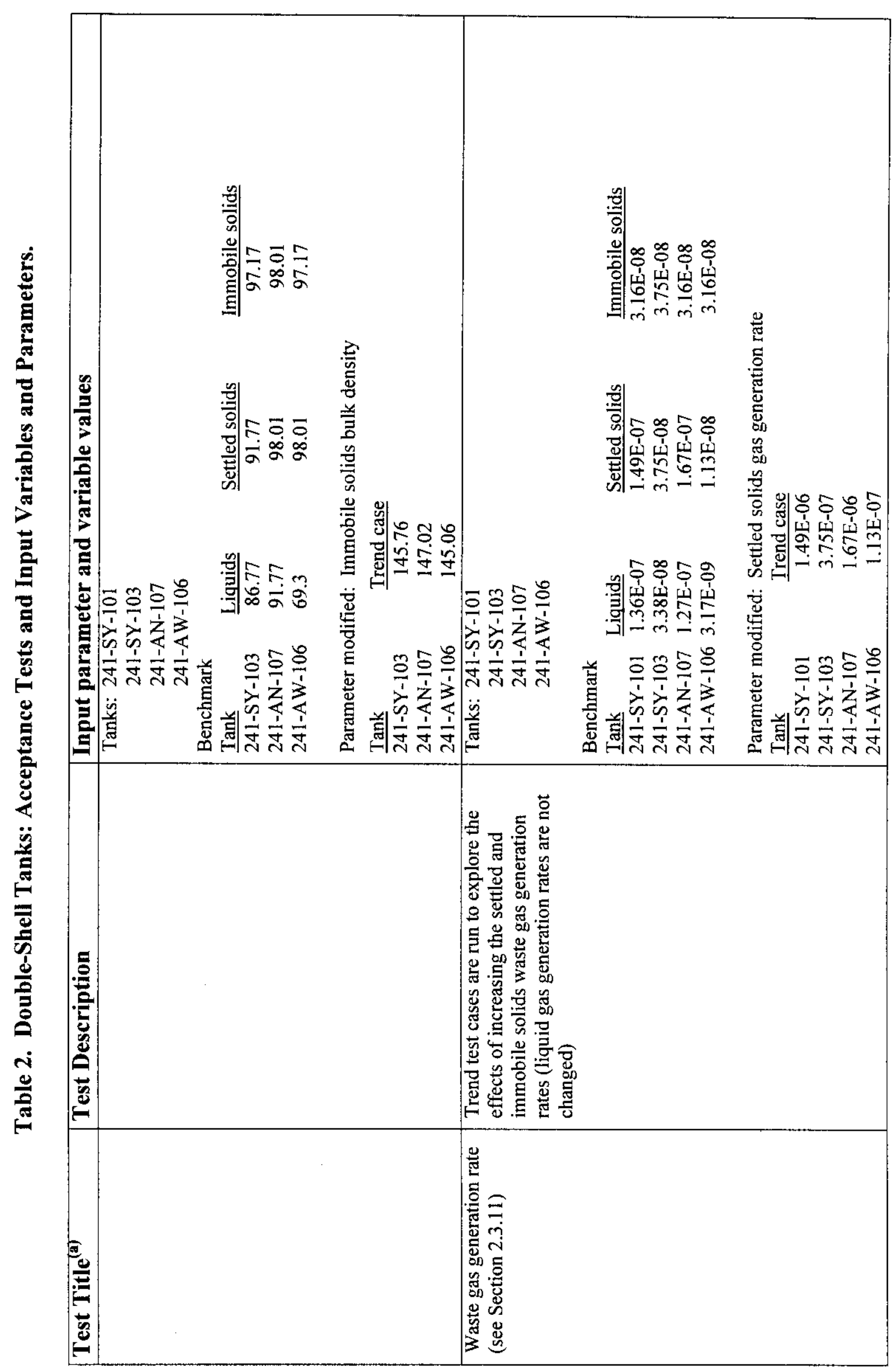


RPP-6888 REV 0

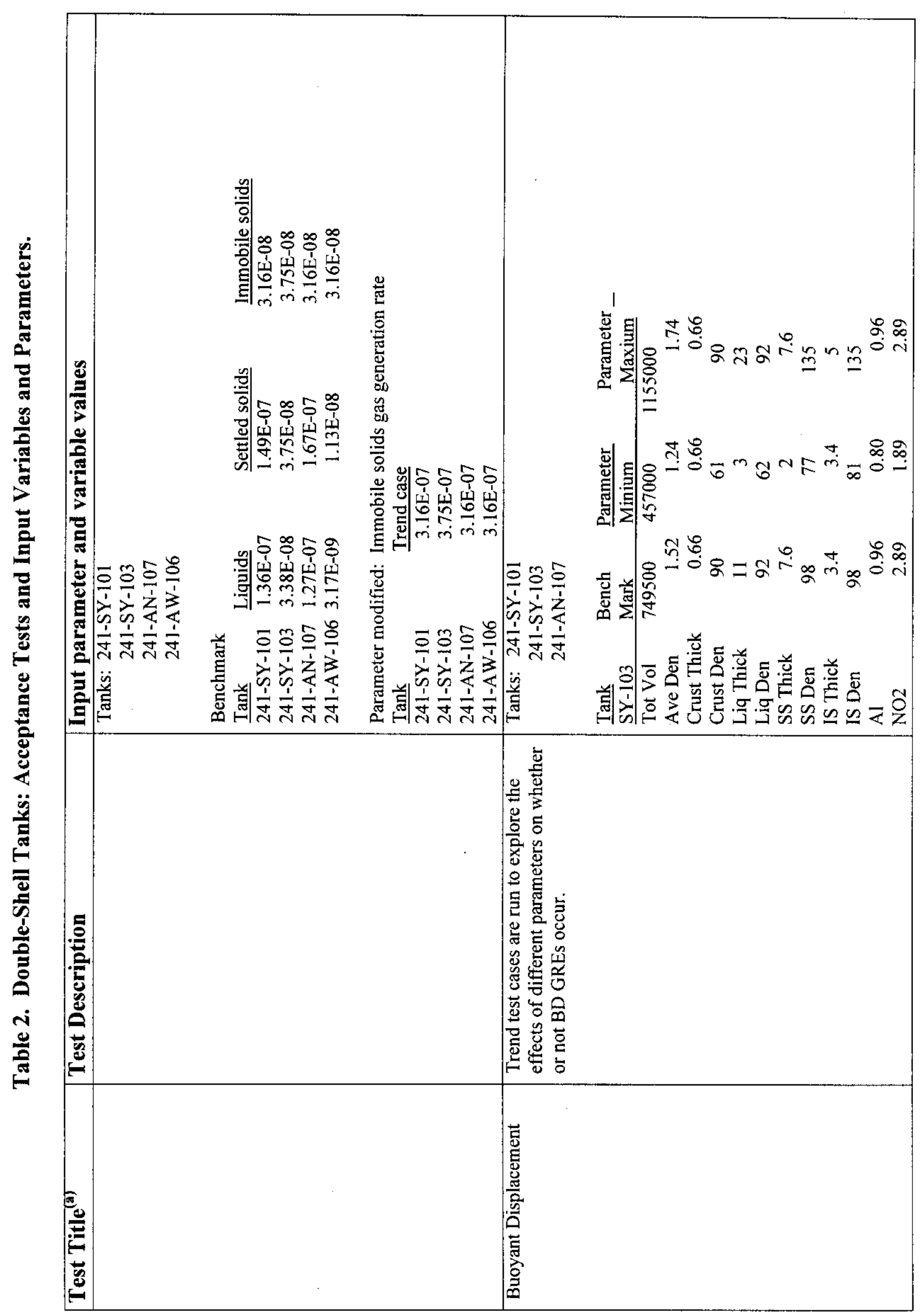




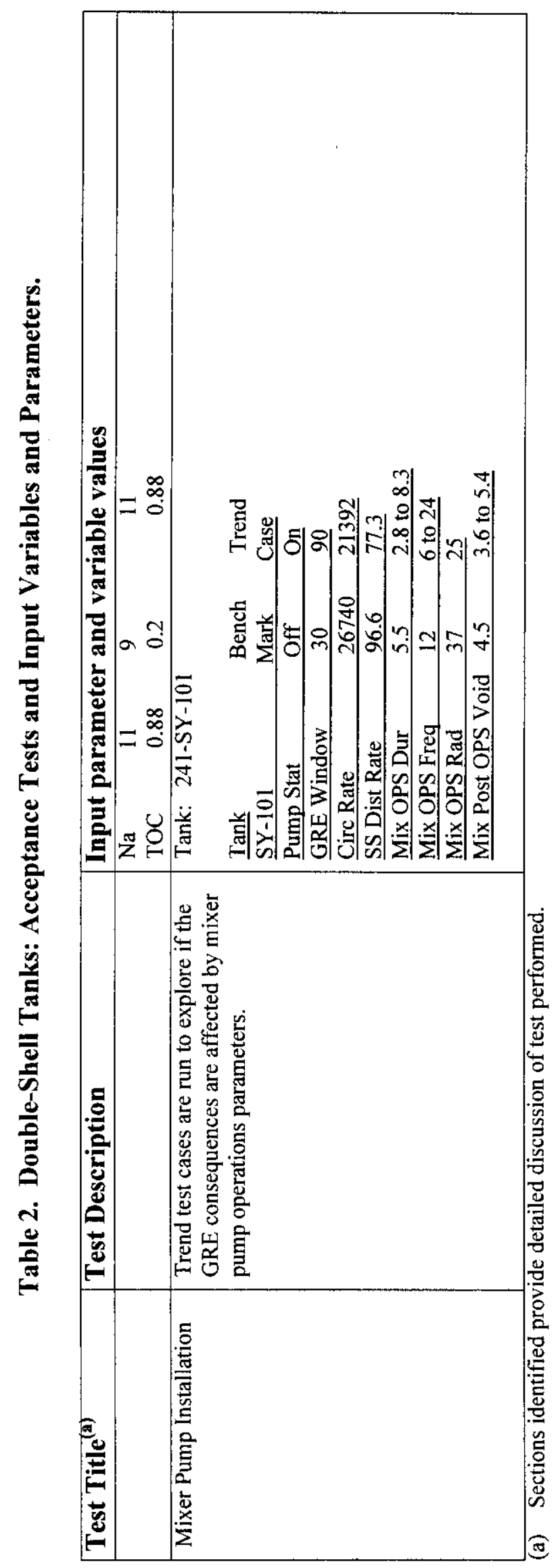

Att-30 


\section{RPP-6888 REV 0}

\subsection{CHARACTERISTIC TESTING}

Characteristic Testing is performed following the successful completion of the "Features Testing." The purpose of the "Characteristic Testing" is to verify that the calculated results are stable and repeatable and to determine the number of trials (or samples) required to achieve stable and repeatable results. These tests are performed at different workstations using the identical statistical analysis parameters. Characteristics Testing is also used to establish system baseline stability or behavior due to changes in statistical analysis parameters. Typically, it would be necessary to test the Analysis Tool as an integrated tool; however, due to the addition of the DST analysis, it is necessary to test both the SST and DST stability. The following subsections describe the characteristics testing, which is common to both SSTs and DSTs and the expected changes to the results caused by the testing. Results of Characteristic Testing are summarized in Section 3.2.

To test system stability the variance, standard deviation and the relative standard deviation values are calculated. The relative standard deviation is calculated using two methods 1) dividing the square root of the variance by the average, and 2) dividing the standard deviation by the average. Simply stated, the standard deviation equals the square root of the variance. In order to comply, the values must not vary by more than a factor or two. Translated to the percentile notation common with relative standard deviations, the values cannot be over $200 \%$.

\subsubsection{Transport Testing}

The purpose of the transport test is to verify that the installed versions of Resolve! 2.13 are repeatable on multiple workstations. That is, for a specific tank, given the same input parameters, Resolve! 2.13 will cafculate identical results. An analyst performs two Benchmark case runs using the same tank, the same sample seed value, and the same sample count. Each analyst performs the same case runs at their work station. The mean and the median of the onsite radiological and toxicological results are shown on the GUI and are compared. In addition, the first ten frequency-consequence pairs for the onsite radiological and toxicological results recorded in the analysis output file (.afo) for each run are compared.

This case will be the Benchmark Case. All new installations will be required to perform the same case and verify the results of the analysis at the new work station are identical to the benchmark case analysis results. Additionally, it is recommended that each analyst rerun this case prior to performing new analyses. See Tables 1 and 2 for a description of the tests performed.

\subsubsection{Sample Count Stability}

This test is used to establish the stability of Resolve! 2.13. That is, changing the number of sample analyzed will impact the statistical analysis and the reported results. It is expected that the reported results for each case based on the number of samples analyzed will vary; however, the variance should not be significant. An analyst performs 17 benchmark runs using a single tank and the same sample seed value. For each run, the sample count is iterated from 200 to 1000 in increments of 50.

The median of the onsite radiological and toxicological results as shown on the GUI, for 17 runs, are compared. The relative standard deviation should be less than $200 \%$. See Tables 1 and 2 for a description of the tests performed. 


\section{RPP-6888 REV 0}

\subsubsection{Sample Seed Stability}

This test is also used to establish the stability of Resolve! 2.13 , similar to the Sample Count Sensitivity test discussed above. That is, changing the seed value will impact the statistical analysis and the reported results. It is expected that the reported results for each sample seed value analyzed will vary; however, the variance should not be significant. An analyst performs 30 benchmark runs using a single tank and the same sample count. For each run, the sample seed is iterated from $100,000,000$ to $970,000,000$ in increments of $3,000,000$.

The median of the onsite radiological and toxicological results are shown on the GUI, for all 30 runs, are compared. The relative standard deviation should be less than 200 . See Tables 1 and 2 for a description of the tests performed.

\subsubsection{Sample Count and Sample Seed Stability}

This test is also used to establish the stability of Resolve! 2.13 , similar to the Sample Count and Sample Seed Sensitivity tests discussed above. That is, changing the sample count and the sample seed value will impact the statistical analysis and the report results. It is expected that the reported results for each case will vary; however, the variance should not be significant. A total of 30 runs will be performed. That is for 10 different sample seeds, 3 different sample counts are analyzed See Tables 1 and 2 for a description of the tests performed.

The median of the onsite radiological and toxicological results as shown on the GUI, for all 30 runs, are compared. The relative standard deviation should be less than $200 \%$.

\subsubsection{Tank Characteristics Testing}

The purpose of the tank characteristics test is to verify that tanks with the same characteristics are evaluated the same in Resolve! 2.13. That is, for two tanks, given the same input parameters and characteristics, Resolve! 2.13 will calculate identical results. As a basis for this test, the analyst uses the tank characteristics (e.g., fill, waste type) as the benchmark case tank (SX-103) to re-characterize a tank from another tank farm. The analyst performs a sensitivity analysis using the same tank characteristics, the same sample seed value, and the same sample count used in the Transport Test. The mean of the onsite radiological and toxicological results as shown on the GUI are compared. See Tables 1 and 2 for a description of the tests performed.

\subsection{TREND TESTING}

Trend testing is performed to evaluate the effects of changes in the pedigreed data values and the predefined controis on the frequency and consequences of a flammable gaseous release. The trend testing is performed by comparing the Benchmark case to the modified (i.e., changed, pedigreed data values and predefined controls) case. As discussed previously (Section 1.1), the major improvement of the refined safety analysis tool is the ability to analysis and evaluate DSTs. Additional changes to the refined safety analysis tool include the buoyant displacement model, the revised mass balance, waste intrusive equipment burns, the mixer pump, waste transfers, and the GUI. Four representative SSTs and DTSs were selected for trend analysis. Tables 2 and 3 for SSTs and DSTs, respectively, identify the tanks and the parameters selected for trend testing. 
Table 3. Summary of Single Shell Tanks Used in Trend Testing.

\begin{tabular}{|c|c|c|c|c|c|c|}
\hline Tank & $\begin{array}{c}\text { Tank volume } \\
\text { (kgal) }^{(\mathbf{a})}\end{array}$ & $\begin{array}{c}\text { Waste } \\
\text { volume } \\
\text { (kgal) }^{(\mathbf{a})}\end{array}$ & Fill factor & $\begin{array}{c}\text { Saltwell } \\
\text { pump status }\end{array}$ & $\begin{array}{c}\text { Ventilation } \\
\text { flow rate } \\
(\mathbf{c f m})\end{array}$ & $\begin{array}{c}\text { GRE } \\
\text { behavior }^{(\mathbf{b})}\end{array}$ \\
\hline $241-\mathrm{B}-111$ & 530 & 237 & $\begin{array}{c}0.3 \\
(\text { medium })\end{array}$ & never pumped & 0.3 & NBD \\
\hline $241-\mathrm{S}-102$ & 758 & 549 & $\begin{array}{c}0.5 \\
\text { (medium) }\end{array}$ & never pumped & 0.2 & NBD \\
\hline $241-\mathrm{U}-111$ & 530 & 329 & $\begin{array}{c}0.4 \\
\text { (medium) }\end{array}$ & never pumped & 0.2 & NBD \\
\hline $241-\mathrm{T}-203$ & 38 & 35 & $\begin{array}{c}0.9 \\
\text { (high) }\end{array}$ & never pumped & 0.4 & NBD \\
\hline
\end{tabular}

(a) Volumes shown are approximates. $\mathrm{kgal}=$ thousand gallons

(b) GRE - Gas release event

BD - Buoyant displacement

NBD - Non-buoyant displacement

NGRE - No observed GRE's

Table 4. Summary of Double Shell Tanks Used in Trend Testing.

\begin{tabular}{|c|c|c|c|c|c|c|}
\hline \multirow[t]{2}{*}{ Tank } & \multirow{2}{*}{$\begin{array}{c}\text { Waste } \\
\text { volume }^{(a)}\end{array}$} & \multicolumn{3}{|c|}{ Waste characteristics $^{\left({ }^{(1)}\right)}$} & \multirow{2}{*}{$\begin{array}{l}\text { Ventilatio } \\
\text { n flow } \\
\text { rate } \\
\text { (cfm) }\end{array}$} & \multirow{2}{*}{$\begin{array}{c}\text { GRE } \\
\text { behavior }^{(\mathfrak{c})}\end{array}$} \\
\hline & & $\begin{array}{c}\text { Volume } \\
\left(\mathrm{ft}^{3}\right)\end{array}$ & $\begin{array}{c}\text { Gas } \\
\text { generation } \\
\text { rate }\left(\text { moles } / \mathbf{m}^{3}\right. \\
\text { sec) }\end{array}$ & $\begin{array}{c}\text { Bulk density } \\
\left(\mathbf{l b m} / \mathrm{ft}^{3}\right)\end{array}$ & & \\
\hline \multirow[t]{4}{*}{$241-A W-106$} & \multirow[t]{4}{*}{579,300} & $\mathrm{C}-0$ & C-NA & C-NA & \multirow[t]{4}{*}{151} & \multirow[t]{4}{*}{ NBD } \\
\hline & & L-46,960 & $\mathrm{L}-3.17 \mathrm{E}-09$ & $\mathrm{~L}-69.3$ & & \\
\hline & & SS-30,480 & SS-1.13E-8 & SS-98.01 & & \\
\hline & & IS-0 & IS-NA & IS-NA & & \\
\hline \multirow{4}{*}{$241-\mathrm{AN}-107$} & \multirow{4}{*}{$1,050,900$} & $\mathrm{C}-0$ & C-NA & C-NA & \multirow[t]{4}{*}{126} & \multirow[t]{4}{*}{ NBD } \\
\hline & & L- 107,490 & L-1.27E-07 & L-86.77 & & \\
\hline & & SS-33,000 & SS-1.67E-07 & SS-91.77 & & \\
\hline & & IS-0 & IS-NA & IS-NA & & \\
\hline \multirow[t]{4}{*}{$241-S Y-101$} & \multirow[t]{4}{*}{$1,107,110$} & $C-14,710$ & C-8.78E-08 & $\mathrm{C}-84.3$ & \multirow[t]{4}{*}{$\overline{485}$} & \multirow[t]{4}{*}{$\mathrm{BD}$} \\
\hline & & L-76,560 & $\mathrm{L}-1.36 \mathrm{E}-07$ & L-98.01 & & \\
\hline & & SS- 56,680 & SS-1.49E-07 & SS-106.13 & & \\
\hline & & IS-0 & IS-NA & IS-NA & & \\
\hline \multirow[t]{4}{*}{$241-S Y-103$} & \multirow[t]{4}{*}{749,200} & $\mathrm{C}-2,920$ & $\mathrm{C}-4.27 \mathrm{E}-08$ & $\mathrm{C}-89.93$ & \multirow[t]{4}{*}{99} & \multirow[t]{4}{*}{$\mathrm{BD}$} \\
\hline & & $\mathrm{L}-48,860$ & $\mathrm{~L}-3.38 \mathrm{E}-08$ & L-91.77 & & \\
\hline & & SS-33,490 & SS-3.75E-08 & SS-98.01 & & \\
\hline & & IS- 14,930 & IS-3.75E-08 & IS-98.01 & & \\
\hline
\end{tabular}

NA - Not applicable

(a) Volumes shown are approximate.

(b) Volumes shown are approximate. $\mathrm{lbm} / \mathrm{cuft}=$ pound moles per cubic foot

C - crust layer

$\mathrm{L}$ - liquid layer

SS - settled solids layer

IS - immobile solids layer

(c) GRE - Gas release event

BD - Buoyant displacement

NBD - Non-buoyant displacement

NGRE - No observed GRE's 


\section{RPP-6888 REV 0}

The general approach followed in trend testing is to develop a Benchmark case for each tank maximizing the sensitivity of the program and to perform multiple analyses or sensitivity case runs for each tank changing only one parameter (i.e., pedigreed data value or predefined control) per analysis. The output from each run or analysis (e.g., frequency and/or consequences) are tabulated and compared. This approach will test the effects of significantly increasing or decreasing a parameter.

Tables 1 and 2 for SSTs and DSTs, respectively, provide a listing of the input parameters and values that are changed for each test. Each trend test uses the Benchmark Case as a template. That is, the Benchmark Case file is "opened" and modified. For all trend tests the Analysis Type is changed from "Benchmark" to "Sensitivity" and the appropriate input parameters and values are changed to the values specified for each test.

The following subsections describe the trend testing. The expected results due to the parameter changes are shown in Tables 5 and 6 for SSTs and DSTs, respectively. Based on the expected results specific results (e.g., frequency, deflagrations, detontations) are compared for each trend test to the benchmark case results.

\subsubsection{Saltwell Pumping}

This test applies to SSTs only. Trend testing cases are run to explore the effect of saltwell pumping on risk. Only the saltwell pupming status is varied; the waste volume, which would change if a tank were actually saltwell pumped, is left unchanged. The influence of saltwell pumping on gas retention characteristics was an elicited parameter (Slezak and Bratzel 1997). Tanks identified as "never pumped" are changed to "previously pumped" and vice versa. Changing the saltwell pumping status from "never pumped" to "previously pumped" is expected to decrease risk. Changing the status to "previously pumped" changes the waste void fraction and GRE frequency, elicitation. The end result should be a reduction in the number of flammable events, frequency and magnitude of radiological and toxicological consequences. Table1 provides a listing of the input parameters and values that are changed for each test.

\subsubsection{Waste Volume}

This test applies to SSTs and DSTs. Trend testing cases were run to explore the effect of changing the waste volume on risk. The waste volume is changed significantly. A reduction in the waste volume will increase the headspace volume. This effect may impact the ability of the headspace gases to reach the lower flammability limit. To test the effect of reducing waste volume, 30 percent of the existing waste was removed. Reducing the waste volume decreases the retained gas volume by providing less waste in which the gas can be stored. In addition, reducing the waste volume increases the headspace volume in which to dilute the GRE gases. Therefore, the size of the GREs and the fraction of the GREs that produce flammable conditions would decrease, which would result in a decrease in the number of events. Due to the properties of the waste the impacts on the frequency and consequences are indeterminate. See Tables 1 and 2 for SSTs and DSTs, respectively, for test input parameters. Therefore DST volume is reduced but is taken from liquids only.

\subsubsection{Number of Intrusive Operations}

This test applies to SSTs and DSTs. Trend testing cases are run to explore the effect of increasing the number of equipment insertion and/or removal operations on risk. The number of operations is increased by a factor of five over the default value. It is expected that the increase in the number of operations increases the risk by increasing the frequency of induced GREs and increasing the frequency of ignition sources. Only the frequency is expected to increase. There should be no change in the number of 


\section{RPP-6888 REV 0}

flammable events and the radiological and toxicological consequences. See Tables 1 and 2 for SSTs and DSTs, respectively, for test input parameters.

\subsubsection{Ignition Control Set}

This test applies to SSTs and DSTs. Trend testing cases were run to explore the effect of changing the ignition controls on risk. It is expected that frequency increases as the level of ignition control is decreased from "Control Set 1" or "Control Set 2" (HNF-1999) to "Past Practices" (i.e., no controls). The ignition control sets, "Control Set 1," "Control Set 2" and "Past Practices" are described in Appendix B of Slezak and Bratzel (1997). Because the controls do affect the GREs, the controls are expected to affect the frequency but have little effect on the radiological and toxicological consequences. All tanks evaluated in the trend test have Ignition Control Set 2. It is expected that changing controls from Ignition Control Set II to Past Practices should not change the number of flammable events or consequences; however, the frequency should increase. See Tables 1 and 2 for SSTs and DSTs, respectively, for test input parameters.

\subsubsection{Ventilation Rate}

This test applies to SSTs and DSTs. Trend testing cases were run to explore the effect of changing the ventilation rate (i.e., increasing or decreasing) on risk. This test involves reducing or increasing the default ventilation flow rate by a factor of 10 . It is expected that actively ventilating a tank, compared to passive ventilation could reduce the time at risk and the computed burn pressures, and perhaps even eliminate flammable conditions altogether (Slezak and Bratzel 1997). Conversely reducing the flow rate could increase the time at risk and the computed burn pressure. Therefore, the expected result of increasing the ventilation rate is a decrease in risk, and decreasing the ventilation rate is an increase in risk. As the ventilation flow rate is modified, gases may be swept out of the tank (increased flow rate) or allowed to accumulate in the headspace (decreased flow rate). See Tables 1 and 2 for SSTs and DSTs, respectively, for test input parameters

\subsubsection{Inerting the Tank Headspace, with Nitrogen}

This test applies to SSTs and DSTs. Trend testing cases were run to determine the impact of inerting the headspace. Sufficient inerting of the headspace will increase the size of GREs needed to reach flammability and can prevent combustion of mixtures that are flammable before release (Slezak and Bratzel 1997). Inerting the headspace reduces the oxidizer for the burn typically furnished by air. Inerting the tank headspace should reduce GRE flammable event frequencies and should result in less damage to the tank as well as reducing the dose consequences. Risk is expected to decrease as inerting is applied. The number of hits, as well as the frequency of burns should decrease, with a small reduction in the consequences. See Tables 1 and 2 for SSTs and DSTs, respectively, for test input parameters.

\subsubsection{Tank Failure (Cracking) Pressure}

This test applies to SSTs only. Trend testing cases were run to explore the effect on risk of decreasing or increasing the dome cracking pressure. For this test, the dome cracking pressure is decreased or increased by $50 \%$. Structural capacity and failure evaluations are discussed in Slezak and Bratzel (1997). It is expected that risk will increase as failure pressure is reduced, and conversely decrease as failure pressure is increased. A decrease or increase in the cracking pressure from the default value should, respectively, increase or decrease the consequences but should not affect the frequency. Additionally, the number of flammable events for an increase or decrease in the cracking pressure should not change. For a decrease in the cracking pressure, more material would be released to the environment for lower combustion 


\section{RPP-6888 REV 0}

pressures. Conversely for the same combustion pressures, less material would be released to the environment, thus resulting in lower consequences. See Table 1 for test input parameters

\subsubsection{Tank Failure (Collapse) Pressure}

This test applies to SSTs and DSTs. Trend testing cases were run to explore the effect on risk of decreasing the dome collapse pressure. The dome collapse pressure is reduced to one-haif of the default value. Structural capacities and failure evaluations are discussed in Slezak and Bratzel 1997. It is expected that risk will increase as failure pressure is reduced. Reducing the dome collapse pressure should result in the same conclusions reached in the Tank Failure (Cracking) pressure test (see Section 2.3.7); i.e., the consequences should increase but the frequency should not be affected. Additionally, the number of flammable events for an increase or decrease in the cracking pressure should not change. The risk would therefore increase somewhat. More material would be released to the environment for lower combustion pressures. See Tables 1 and 2 for SST and DSTs, respectively, for test input parameters.

\subsubsection{Waste Intrusive Equipment}

This test applies to SSTs and DSTs. Trend testing cases were run to explore the effect on risk of waste intrusive equipment flammable events. A series of four tests are performed: 1) increase the number of operations per year; 2) change the ignition control from purged to not purged; 3) increase the diameter of the equipment; and 4) increase the length of the equipment. The benchmark case shows no operations thus increasing the number of operations will increase risk. The current ignition control requires purging waste intrusive equipment in accordance with National Fire Prevention Association (NFPA); therefore, not purging the equipment (maintaining operations at 5 per year) the risk will increase over benchmark conditions and test 1 in this series. Tests 3 and 4 of the series should effect the detonation cell size thus an impact is expected on the number of flammable events. However, realistic changes in the equipment design (e.g., diameter and length) may be insufficient to impact the results of Test 1 in this series.

Therefore the expected changes at this time are indeterminate.

\subsubsection{Increase in Bulk Density Ratio of Solids to Liquids}

This test applies to DSTs only. Two tests are performed to determine the relative impacts of increasing the bulk density of the settled solids and immobile solids with respect to GRE behavior. These tests, unlike the previous tests, do not model operations or potential controls that would be implemented in the tank farms. However, based on the behavior of the waste it is anticipated that GRE behavior will be affected. That is, the higher the densities the more gas that will be retained in the waste and released spontaneously or due to some initiating event such as a seismic event or intrusive operation. Thus, it is anticipated that the risk will increase.

\subsubsection{Increase in Waste Gas Generation Rate}

This test applies to DSTs only. Two tests are performed to determine the relative impacts of increasing the gas generation rate in the settled solids and immobile solids with respect to GRE behavior. These tests unlike the previous tests do not model operations or potential controls that would be implemented in the tank farms. It is anticipated that the risk will increase relatively proportional to the increased rates. 


\section{RPP-6888 REV 0}

\subsubsection{Mixer Pump}

This test applies to DSTs only. The tests, in addition to the Features Tests, were performed to determine the impacts of a mixer pump on consequences. Based on operational experience it is well understood that the mixer pump will reduce the efficiency of the GREs thus the consequences should decrease. Similarly, the frequency of buoyant GREs will also decrease. 


\section{RPP-6888 REV 0}

This page intentionally left blank.

Att-38 


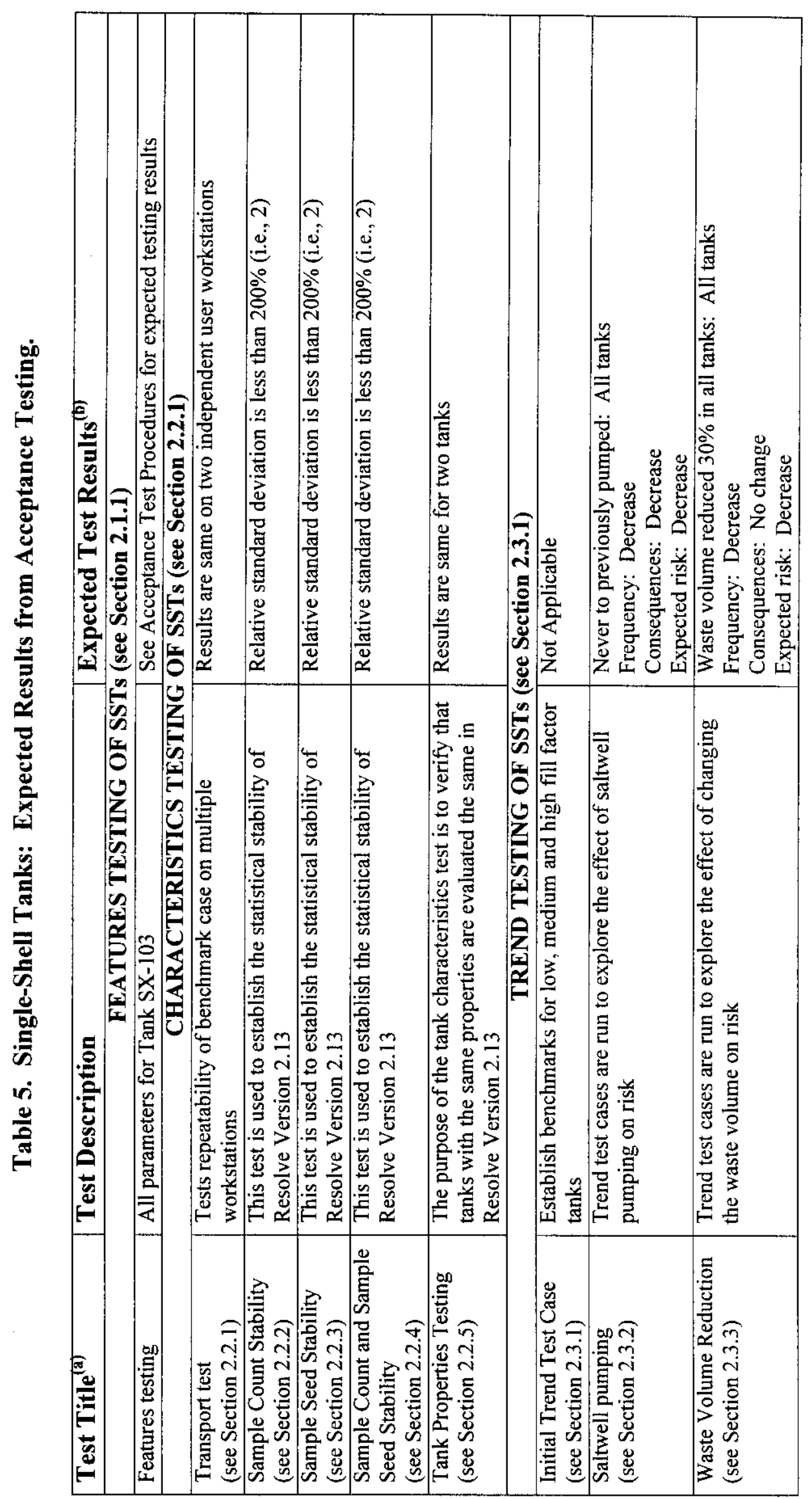




\section{RPP-6888 REV 0}

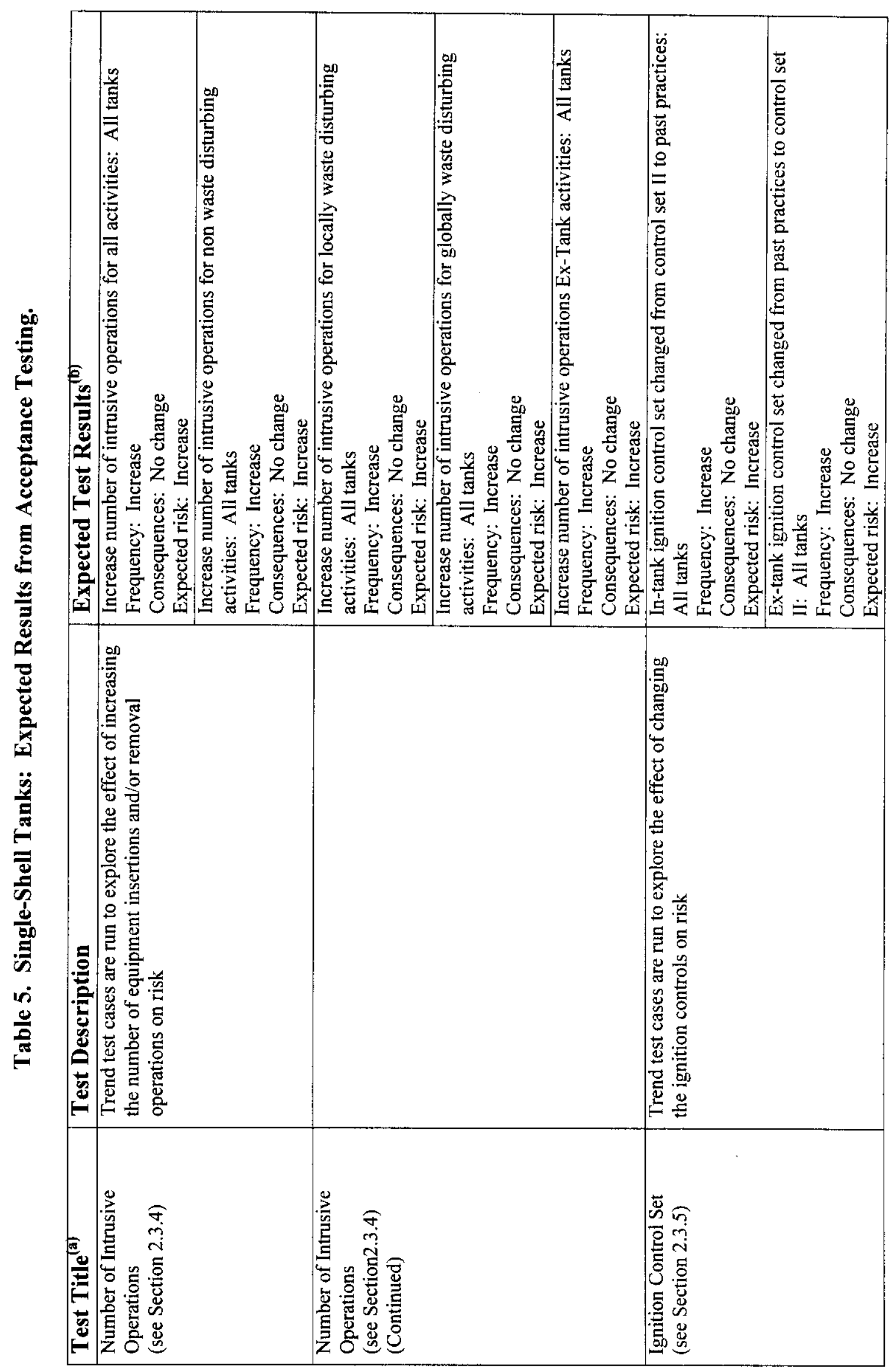




\section{RPP-6888 REV 0}

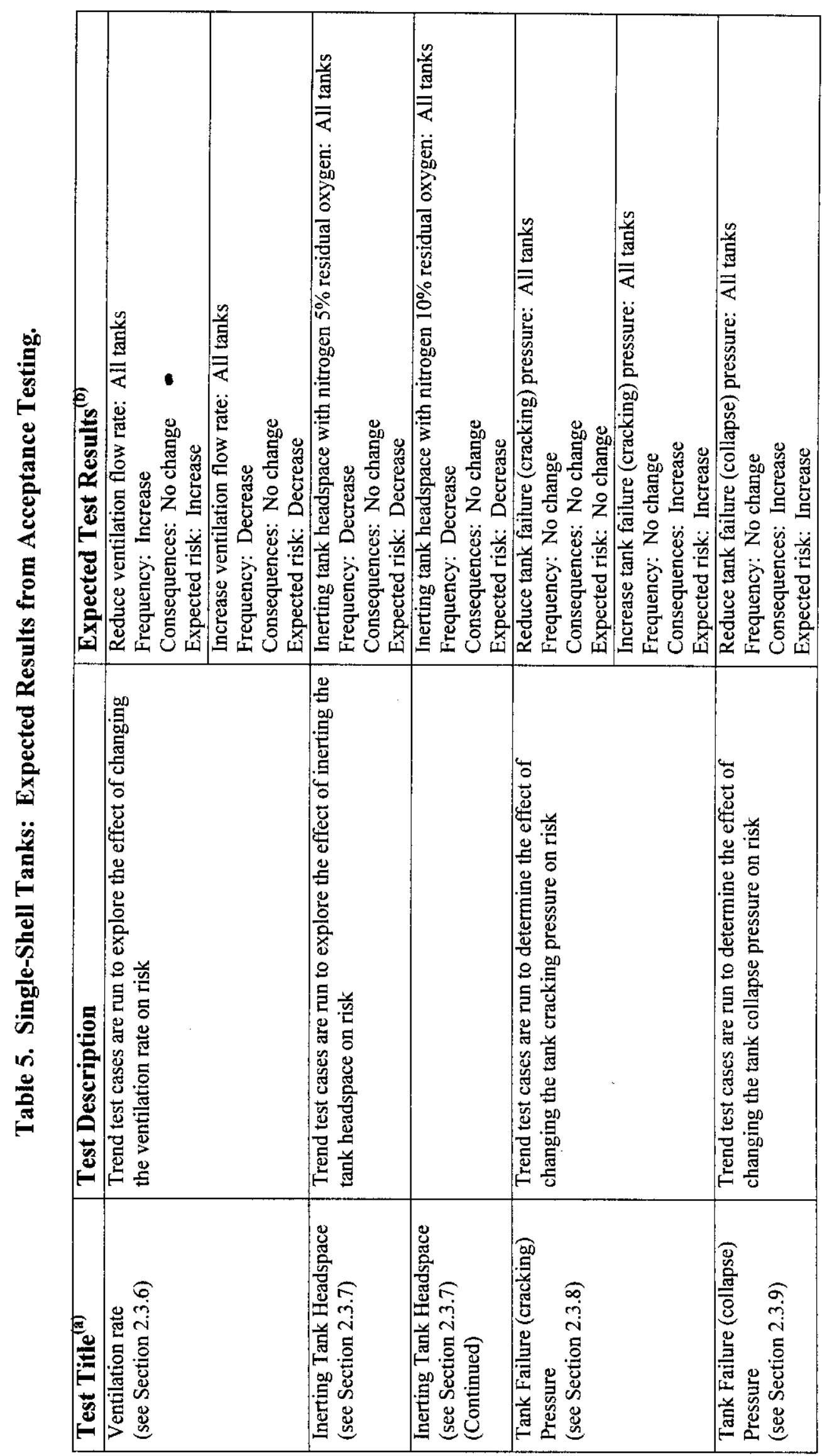


RPP-6888 REV 0

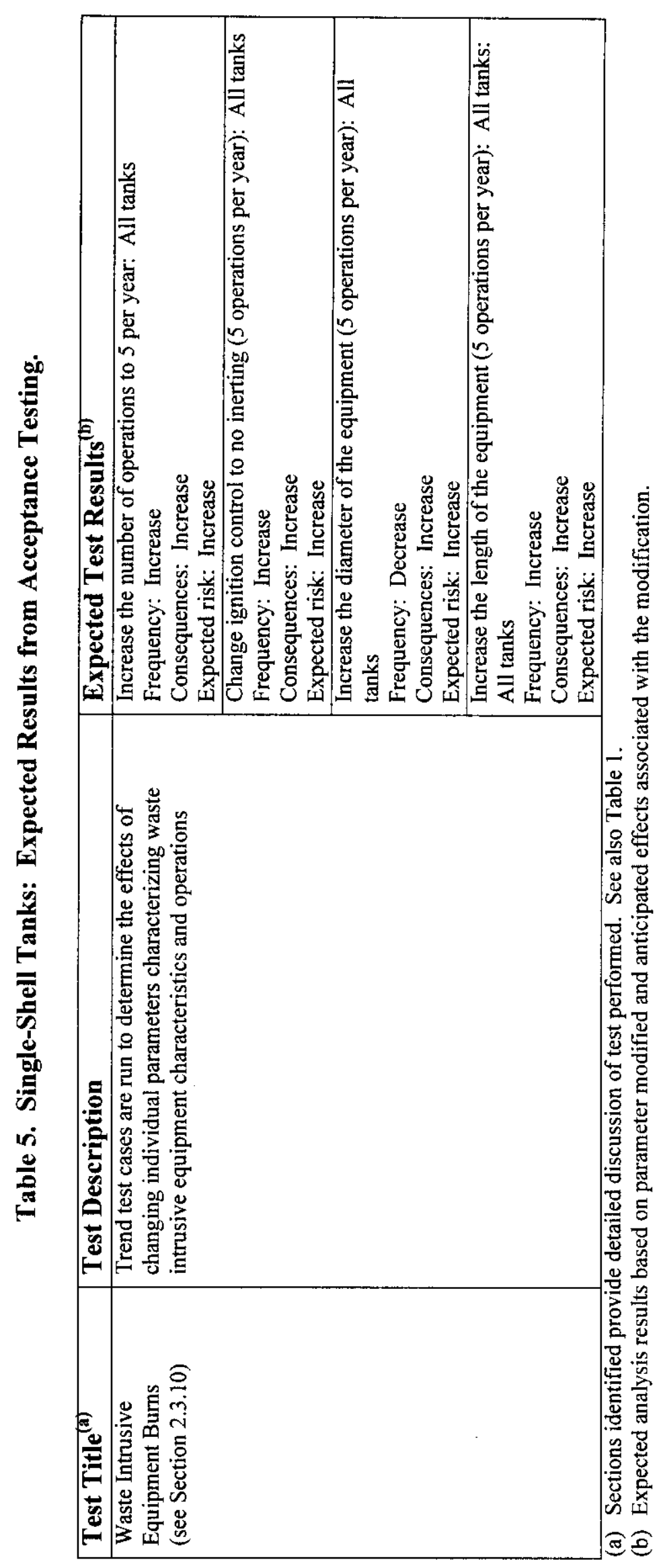




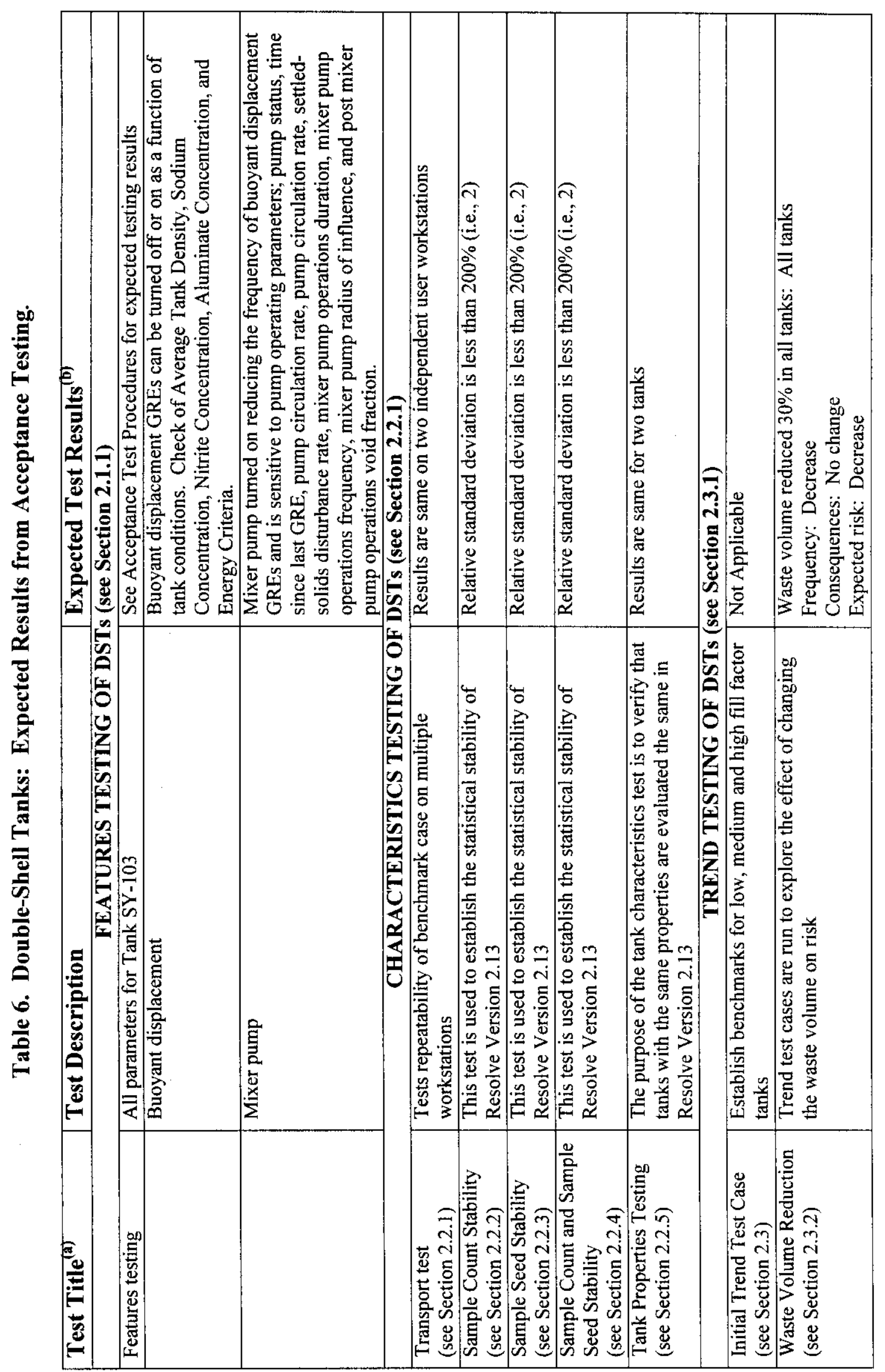


This page cannot be converted.

Please view the native document

for the original page. 
RPP-6888 REV 0

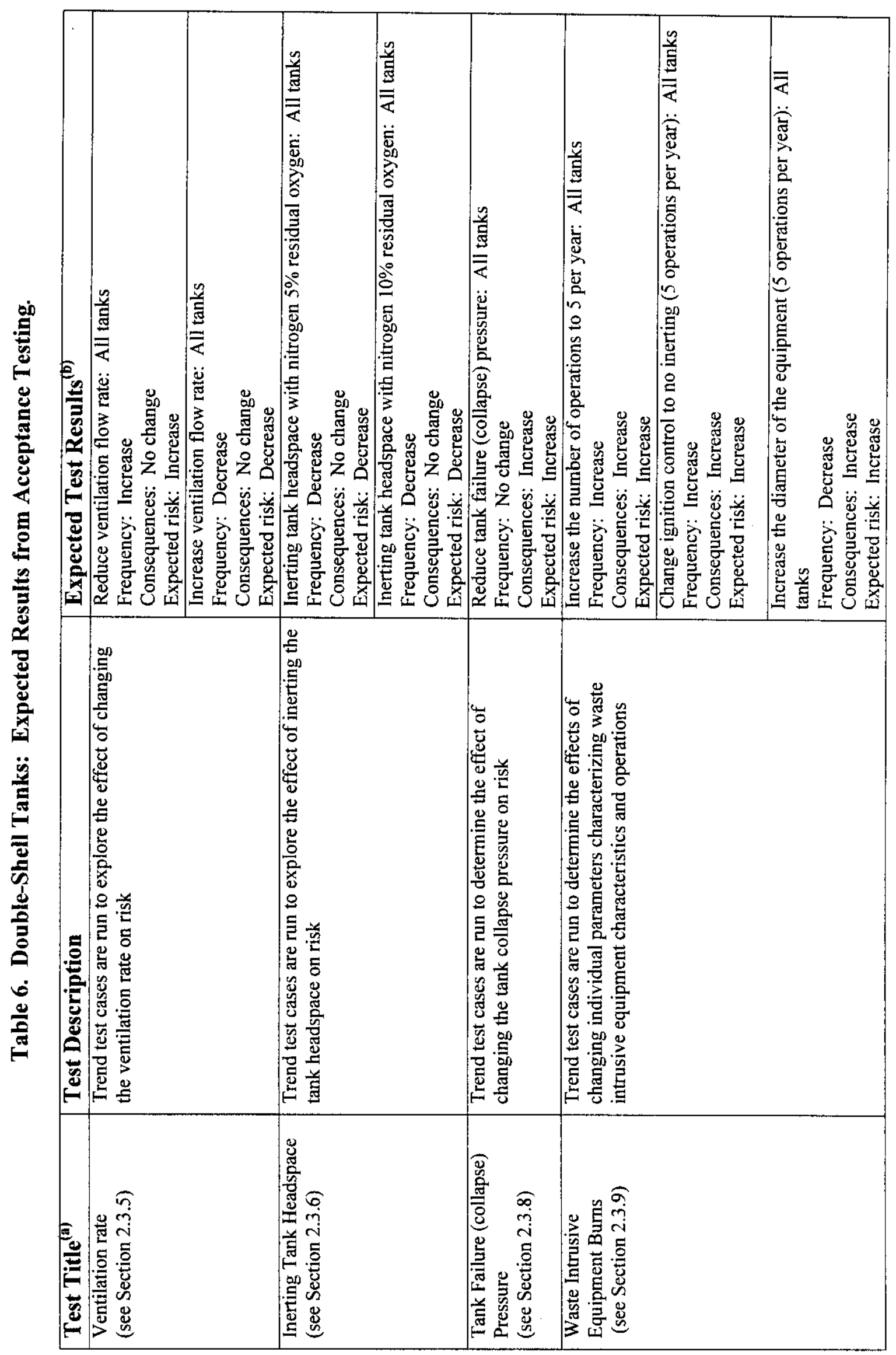


RPP-6888 REV 0

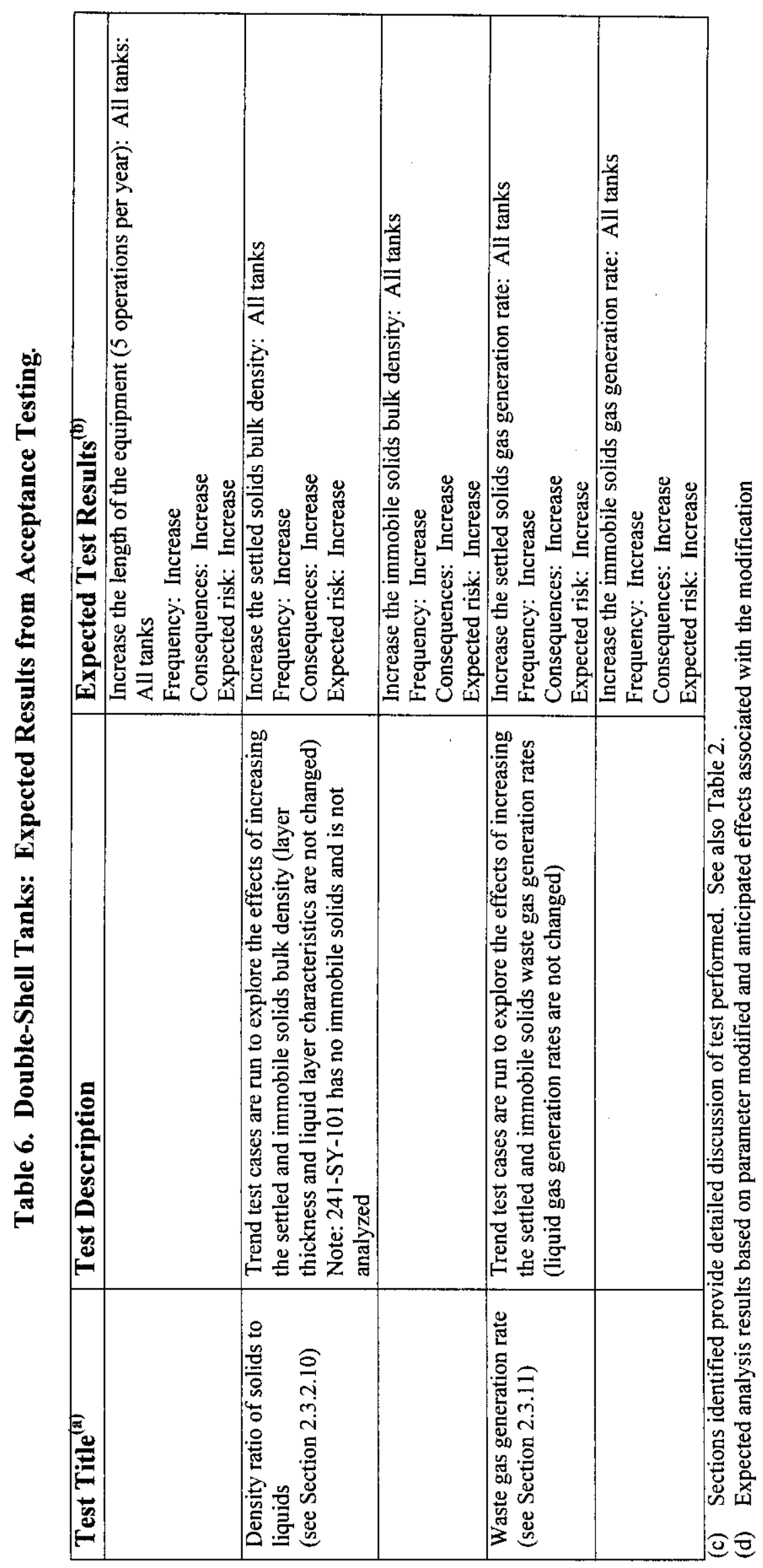


RPP-6888 REV 0

崩

Att-47 


\subsection{SUMMARY OF TEST RESULTS}

The results or conclusions reached regarding the acceptability of version 2.13 or the insights gained during testing are summarized in the following Sections. Appendix $\mathrm{C}$ provides a listing of all discrepancy reports generated during the developmental testing and the Acceptance Tests.

\subsection{FEATURES TESTING}

The Features Test performed were designed to test all facets of the Analysis Tool. For both the SSTs and DSTs this involved testing all of the options available and ensuring the computer code did not "crash" as a result. During the development of this version significant developmental testing was performed by both the TFC Team and SNL personnel. As a result of the developmental testing all features performed as expected.

Additional Features Tests were performed for the DSTs, including turning on and off the mixer pump, buoyant displacement GRE go/no-go tests, waste transfers, and waste intrusive equipment impacts. As stated previously stated, significant developmental testing was performed prior to finalization of Version 2.13. As a result of this testing all features performed as expected.

\subsection{CHARACTERISTICS TEST}

The following table (Table 7) summarizes the results of the Characteristics Tests performed. As can be seen all results meet the established criteria discussed in Section 2.2.

\subsection{SUMMARY OF TREND TESTING RESULTS}

Figures 1 through 11 provide a summary of the trend testing results by tank for each of the parameters modified. Appendices B and C provide the detailed data and qualitative comparisons of the calculated results for the parameter modifications to the benchmark case.

As can be seen from Figures 1 through 8 the majority of the parameters modified for both DSTs and SSTs did not significantly affect the benchmark results. The parameter modifications that did significantly impact the results include waste volume reduction and an increase or decrease in ventilation flow rate. In each of these cases the results trend as expected. Although the majority of the results trended as expected, it should be noted that results for increasing globally waste disturbing activities in SSTs (see Figure 4) did not. This issue has been discussed with the refined safety analysis development team. It was determined at this time that this will be identified as a limitation (see Section 4).

With respect to waste intrusive equipment, as expected (see Figures 9 and 10) for both DSTs and SSTs, increasing the number of waste intrusive activities increased the number of potential deflagrations and detonations when compared to the benchmark case. Additionally, as expected, removing the purge from the waste intrusive equipment significantly increased the number of potential deflagrations and detonations when compared to the base case or five operations per year. Increasing the equipment diameter or length had no impact on the base case results. 
Table 7. Summary of the Results of the Characteristics Tests Performed.

\begin{tabular}{|c|c|c|c|c|c|}
\hline \multirow{2}{*}{$\begin{array}{c}\text { Characteristic } \\
\text { Test }\end{array}$} & \multicolumn{5}{|c|}{ Test results - Relative standard deviation (\%) } \\
\hline & $\begin{array}{c}\text { Number of } \\
\text { events } \\
\text { modeled }\end{array}$ & $\begin{array}{c}\text { Accident } \\
\text { frequency }\end{array}$ & $\begin{array}{l}\text { Radiological } \\
\text { consequences }\end{array}$ & $\begin{array}{l}\text { Toxicological } \\
\text { consequences }\end{array}$ & Expected risk \\
\hline \multicolumn{6}{|c|}{ SSTs - S-102 } \\
\hline Transport & \multicolumn{5}{|c|}{ Results obtained at two workstations for same tank were identical } \\
\hline $\begin{array}{l}\text { Sample count } \\
\text { sensitivity }\end{array}$ & 40.69 & 11.51 & 24.41 & 25.38 & 136.38 \\
\hline $\begin{array}{l}\text { Sample seed } \\
\text { sensitivity }\end{array}$ & 4.20 & 10.39 & 26.20 & 23.40 & 95.75 \\
\hline $\begin{array}{l}\text { Sample count and } \\
\text { sample seed } \\
\text { sensitivity (Count } \\
=1000 \text { ) }\end{array}$ & 3.60 & 7.84 & 12.93 & $\overline{6} \overline{30}$ & 101.57 \\
\hline $\begin{array}{l}\text { Tank } \\
\text { characteristics }\end{array}$ & \multicolumn{5}{|c|}{ Results obtained two tanks characterized the same were identical } \\
\hline Characteristic Test & \multicolumn{5}{|c|}{ Test results - Relative standard deviation (\%) } \\
\hline & $\begin{array}{l}\text { Number of } \\
\text { events modeled }\end{array}$ & $\begin{array}{l}\text { Accident } \\
\text { frequency }\end{array}$ & $\begin{array}{l}\text { Radiological } \\
\text { consequences }\end{array}$ & $\begin{array}{l}\text { Toxicological } \\
\text { consequences }\end{array}$ & Expected risk \\
\hline \multicolumn{6}{|c|}{ DST - SY-103 } \\
\hline Transport & \multicolumn{5}{|c|}{ Results obtained at two workstations for same tank were identical } \\
\hline $\begin{array}{l}\text { Sample count } \\
\text { sensitivity }\end{array}$ & 42.40 & 7.41 & 30.05 & 38.70 & 114.98 \\
\hline $\begin{array}{l}\text { Sample seed } \\
\text { sensitivity }\end{array}$ & 1.43 & 5.54 & 30.47 & 31.71 & 112.60 \\
\hline $\begin{array}{l}\text { Sample count and } \\
\text { sample seed } \\
\text { sensitivity (Count } \\
=1000 \text { ) }\end{array}$ & 1.01 & 3.96 & 38.23 & 40.52 & 45.29 \\
\hline $\begin{array}{l}\text { Tank } \\
\text { characteristics }\end{array}$ & \multicolumn{5}{|c|}{ Results obtained two tanks characterized the same were identical } \\
\hline
\end{tabular}


Figure 11 graphically shows the results obtained due to modifications in the characteristics of the settled solids and the immobile solids (hard pan). As can be seen increasing the ratio of the settled solids to the liquids increased the number of deflagrations in all tanks and in most tanks increased the number of detonations. Of particular interest, an increase in the ratio of settled solids to liquids created buoyant displacement GREs in Tank 241-AW-106 (identified as a non-buoyant tank). As a result of the change the waste passed the energy criteria discussed in Slezak and Bratzel (1999). Also of particular interest is changes in the gas generation rate of the settled solids reduced the potential number of non-buoyant displacement GREs in Tank 241-AW-106. Similar results were obtained in Tank 241-SY-103 when the gas generation rate in the immobile solids was changed.

Not shown graphically are the results obtained from buoyant displacement and mixer pump testing. As expected changes in the five criteria identified in Table 6 (Features Testing) created buoyant displacement GREs in non-buoyant displacement tanks and vice versa. Similarly as expected, the calculated results due to turning on the mixer pump and changing selected parameters to model existing conditions in Tank 241SY -101 were validated to current conditions or observed GRE behavior in the tank.

\subsection{DISCREPANCY TESTING AND LIMITATIONS}

This section discusses discrepancy reports generated during testing and the limitations identified as a result of acceptance testing.

\subsection{DISCREPANCY REPORTS}

All discrepancy reports generated during developmental testing of Resolve! Version 1.51 to Version 2.13 are closed. Appendix A provides a listing of the discrepancy reports generated during developmental testing and acceptance testing. As discussed previously, due to the extensive developmental process and developmental testing with the exception of the limitation identified in Section 4.2, no discrepancy reports were generated as a result of acceptance testing. The discrepancy reports generated during developmental testing, as expected, were due to the significant refined safety analysis Tool modifications to include DSTs, buoyant displacement GRE behavior, and mixer pump modeling.

\subsection{LIMITATIONS/OBSERVATIONS}

As a result of acceptance testing the only limitation identified is associated with increased globally waste disturbing activities in SSTs. As discussed in Section 3.3, the results obtained for the these operations (only frequency) are contrary to expectations. This limitation will be addressed in finalization activities of the refined safety analysis tool.

Additionally, it should be noted that additional testing is required to understand GRE behavior, i.e., efficiency and source of the GRE, and spontaneous versus induced versus seismic and the source impacts on GRE behavior. 


\section{RPP-6888 REV 0}

\subsection{REFRENCES}

Lavender, J. C., B. D. Lacey, and A. B. Webb, 1998, Letter Report: Resolve Version 1.51 Acceptance Testing: Test Plan and Test Results, Pacific Northwest National Laboratory, Richland, Washington.

Slezak, S. E., F. Gelbard, and W. Cheng, 1999, Implmentation Details of the Flammable Gas Refined Safety Analysis Methodology, Sandia National Laboratories, Albuquerque, New Mexico. 
RPP-6888 REV 0

This page intentionally left blank.

Att-52 
RPP-6888 REV 0

APPENDIX A

ALL ARS DISCREPANCY REPORT 
RPP-6888 REV 0

This page intentionally left blank.

Att-A-ii 


\section{RPP-6888 REV 0}

\section{APPENDIX A}

This Appendix provides a listing of all Discrepancy Reports generated by the TFC Team and the refined safety analysis tool development team during development and acceptance testing of Resolve Version 2.13. 
RPP-6888 REV 0

This page intentionally left blank.

Att-A-iv 


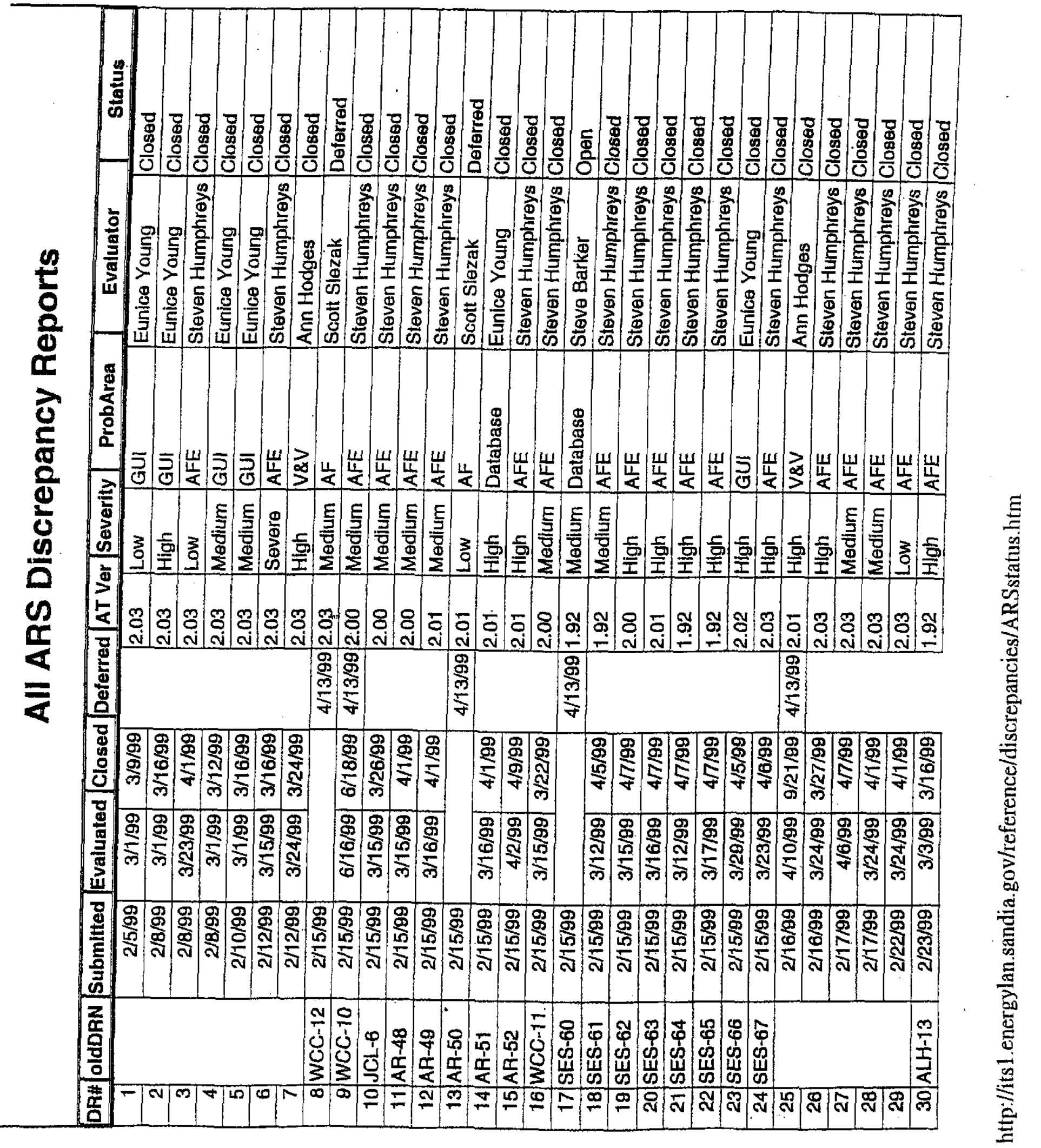




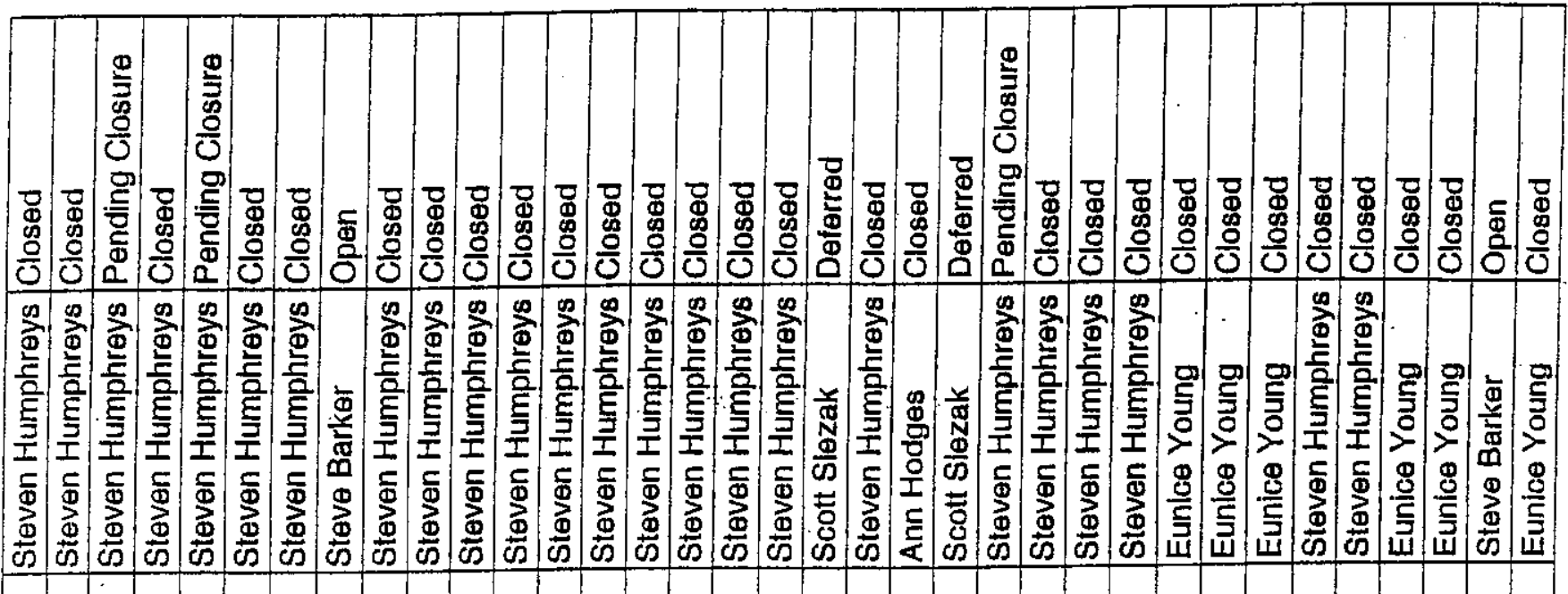

\section{思}

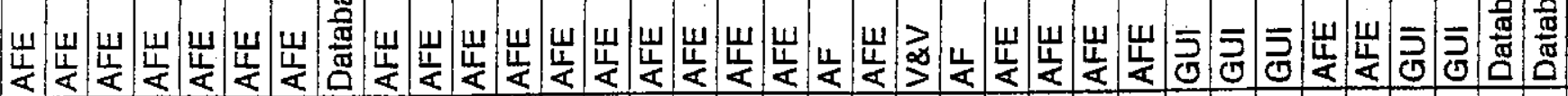
它

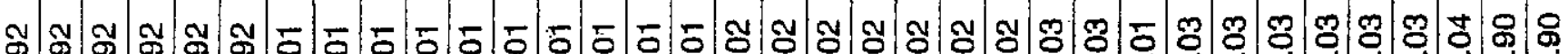

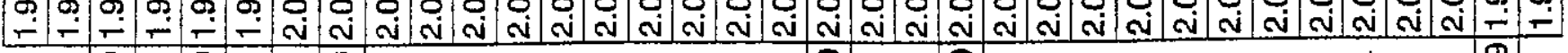

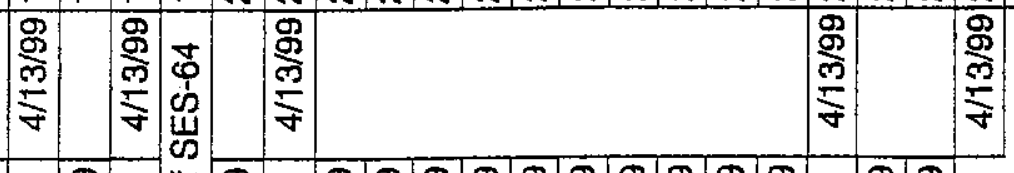

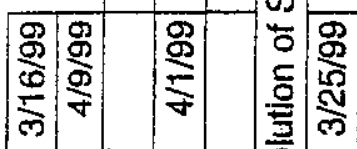

क

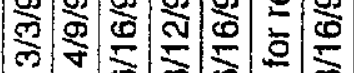

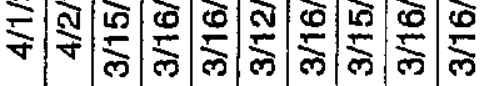

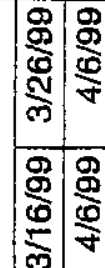

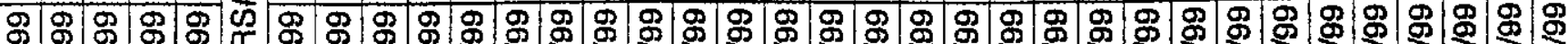
मె

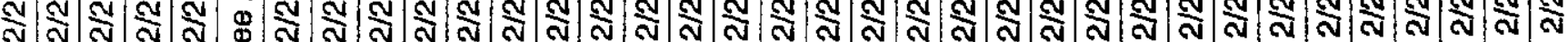

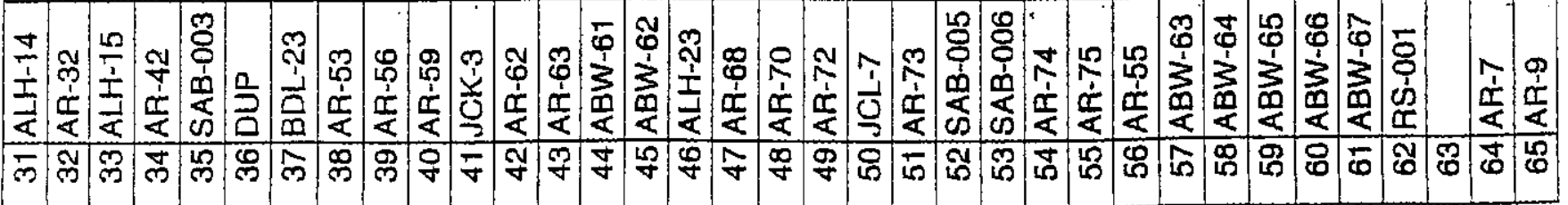



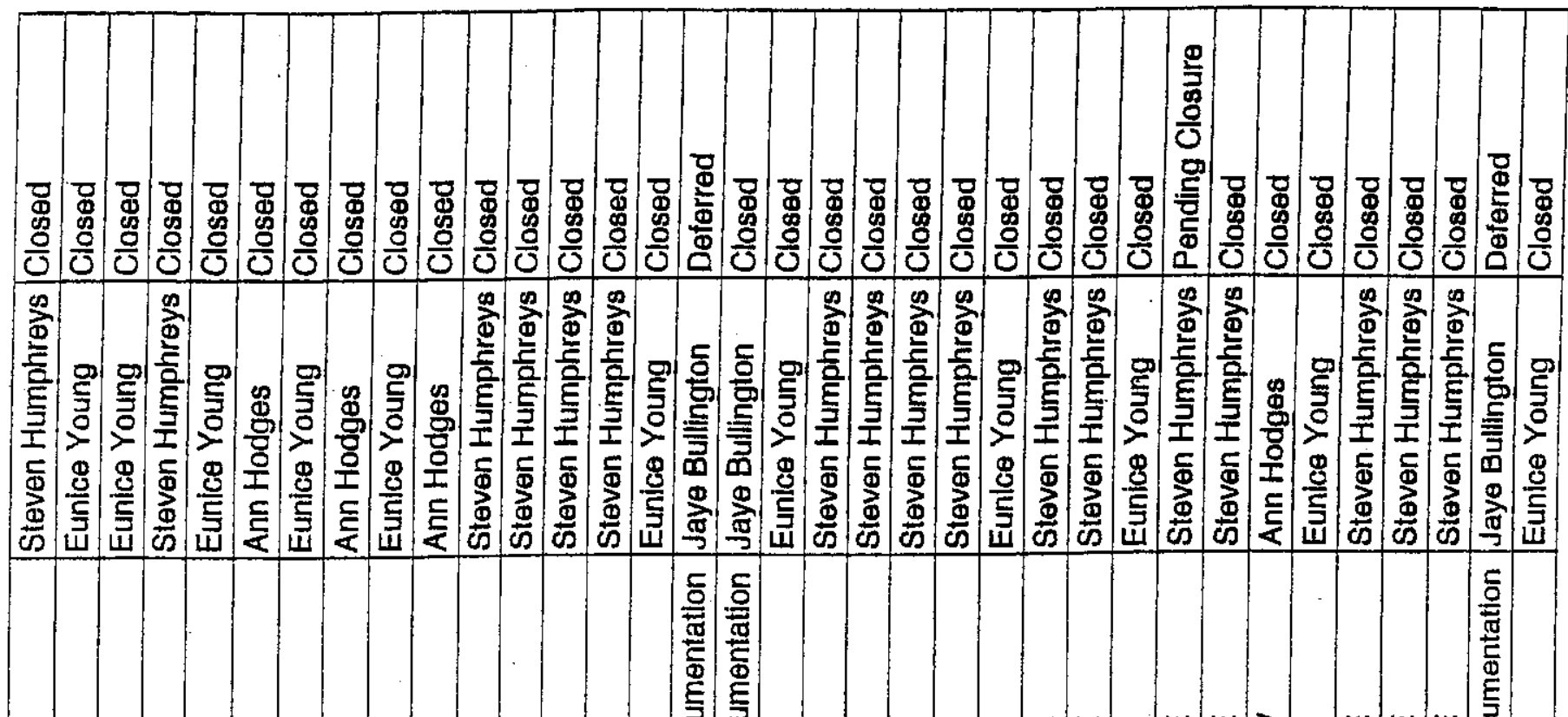

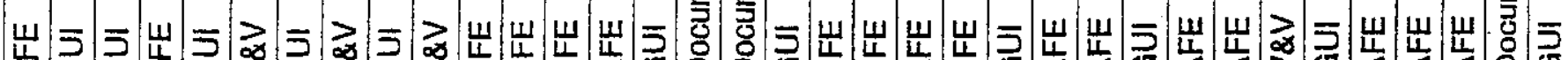
崖 宁

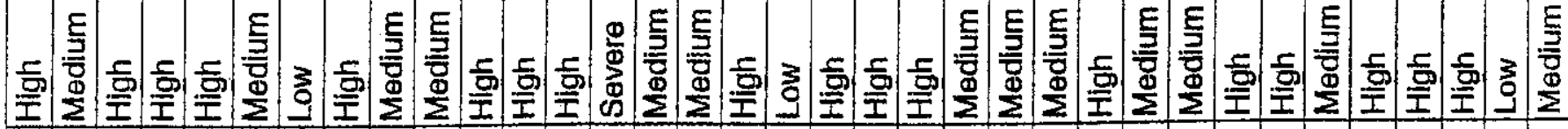

ᄋ요유

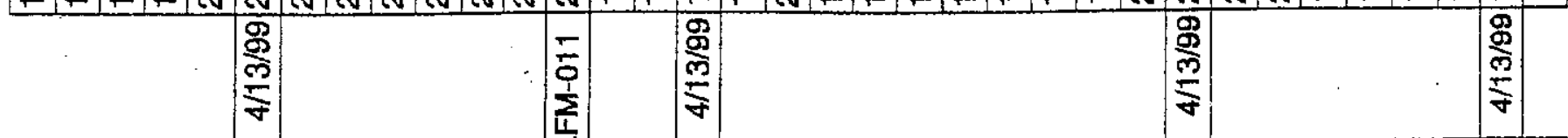

\begin{tabular}{|c|c|c|c|c|c|c|c|c|c|c|c|c|c|c|c|c|c|c|c|c|c|c|c|c|c|c|c|c|c|c|}
\hline$\frac{g}{2}$ & 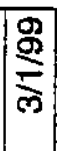 & 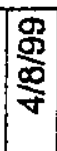 & 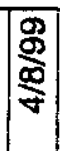 & $\frac{\mathscr{D}}{\frac{D}{\infty}}$ & $\frac{\stackrel{\rho}{\mathrm{S}}}{\mathrm{N}}$ & 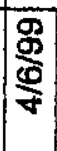 & $\frac{\mathscr{g}}{\stackrel{8}{\rho}}$ & 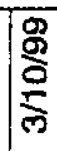 & 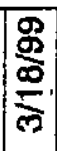 & 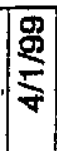 & 息 & ㅎํㅇㅇㅛ & 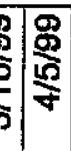 & $\frac{\mathscr{m}}{\mathrm{g}}$ & $\frac{9}{9}$ & $\frac{g}{\frac{9}{2}}$ & 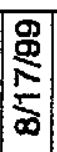 & $\frac{9}{\frac{9}{5}}$ & 密 & $\frac{\operatorname{s}}{\mathbf{g}}$ & & $\begin{array}{l}\frac{9}{9} \\
\text { 号 } \\
\text { v }\end{array}$ & 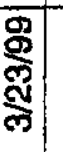 & & $\begin{array}{l}\frac{2}{2} \\
\frac{2}{2} \\
\frac{2}{2}\end{array}$ & 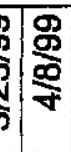 & $\frac{9}{\frac{9}{5}}$ & 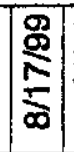 & $\frac{g}{\frac{g}{5}}$ & \\
\hline 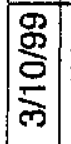 & $\frac{\operatorname{g}}{\underset{\mathrm{g}}{\mathrm{m}}}$ & 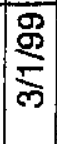 & $\mid \begin{array}{l}\frac{8}{\mathrm{~g}} \\
\frac{\mathrm{O}}{\mathrm{O}} \\
\mathrm{m}\end{array}$ & 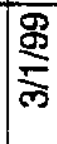 & & $\begin{array}{l}\frac{9}{2} \\
\frac{5}{5} \\
\frac{5}{4}\end{array}$ & $\frac{\mathscr{g}}{\frac{D}{\rho}}$ & $\frac{2}{m}$ & $\begin{array}{l}\frac{9}{\mathrm{~g}} \\
\frac{\mathrm{d}}{\mathrm{m}}\end{array}$ & 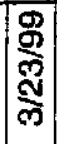 & 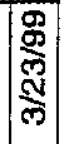 & 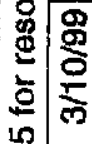 & 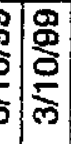 & $\begin{array}{l}\frac{g}{\mathrm{~g}} \\
\frac{\mathrm{d}}{\mathrm{D}} \\
\mathrm{m}\end{array}$ & 胥 & $\frac{9}{\stackrel{9}{2}}$ & 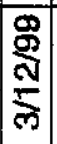 & 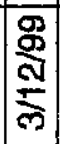 & के & 昰 & 离 & 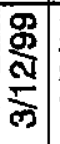 & 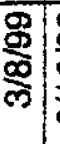 & 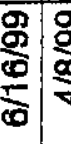 & $\frac{g}{\stackrel{g}{9}}$ & $\frac{\rho}{\frac{\rho}{\rho}}$ & 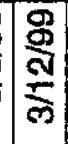 & 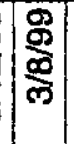 & 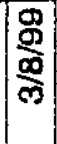 & \\
\hline
\end{tabular}

த ڤ్ర

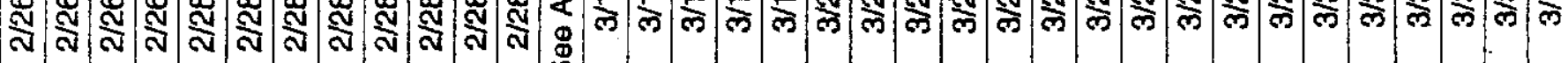

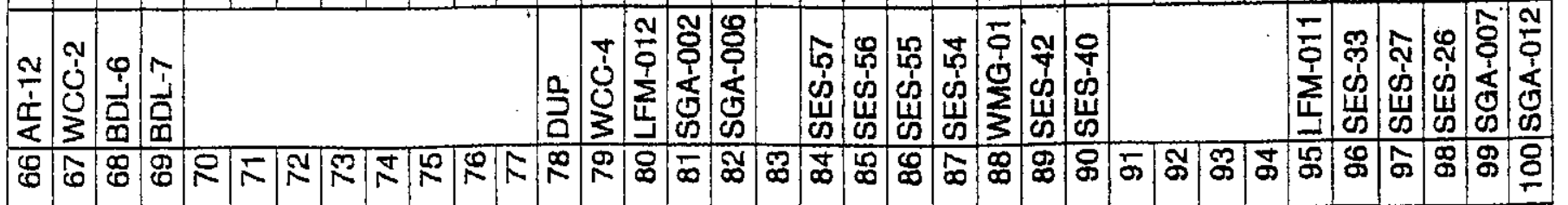



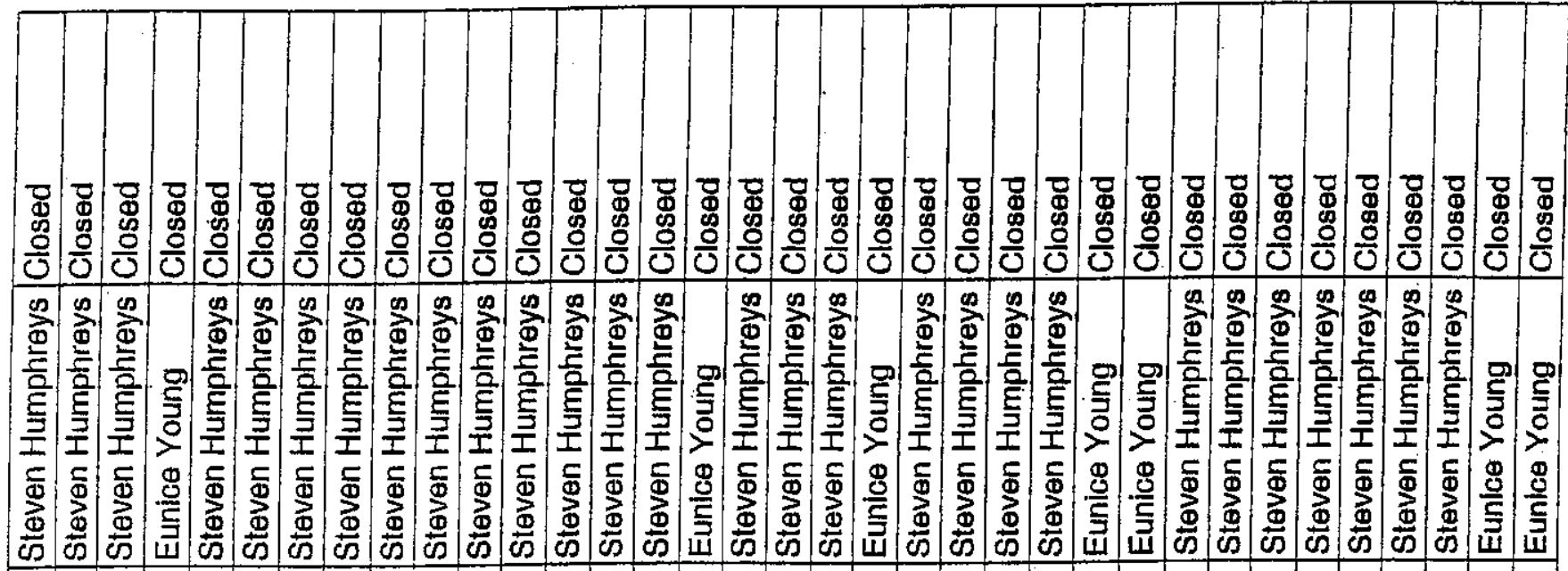

$\$$

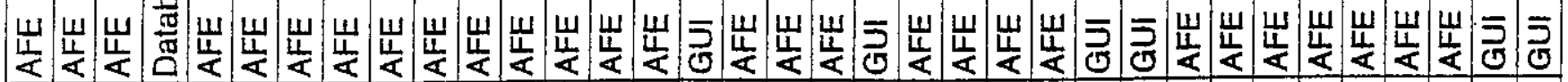

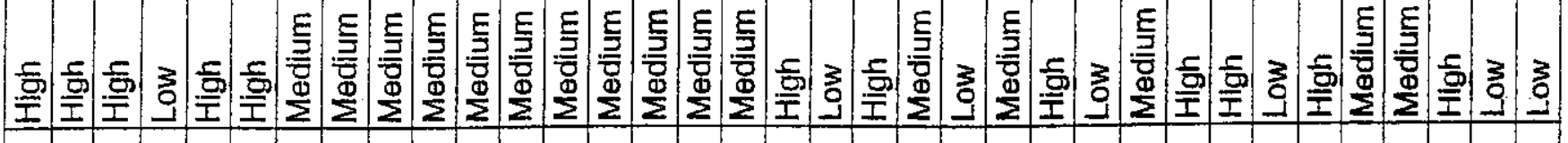

ภำ ส

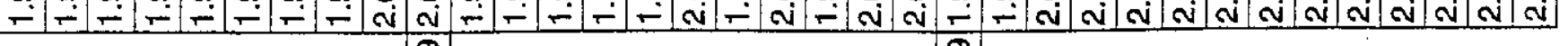

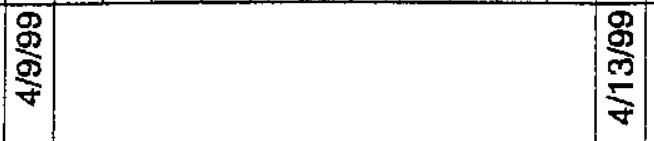

துத:த

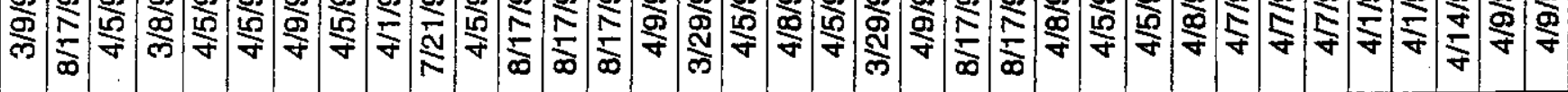
இ இ ली

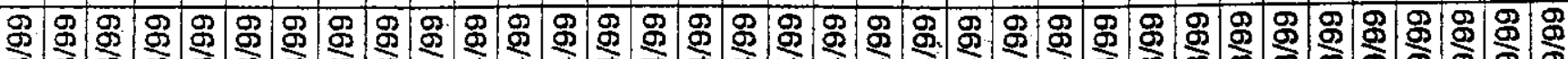

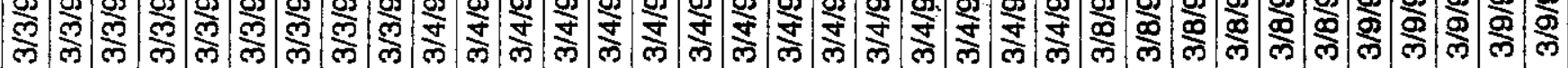

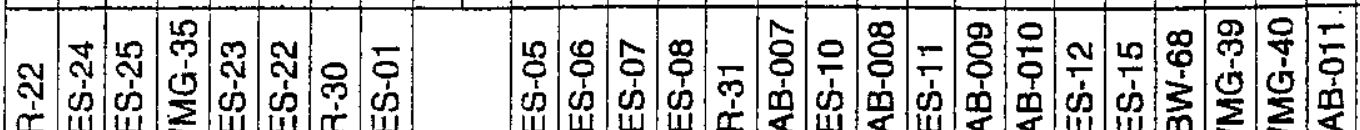

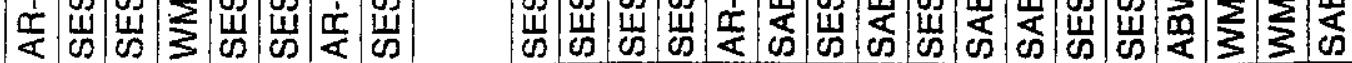



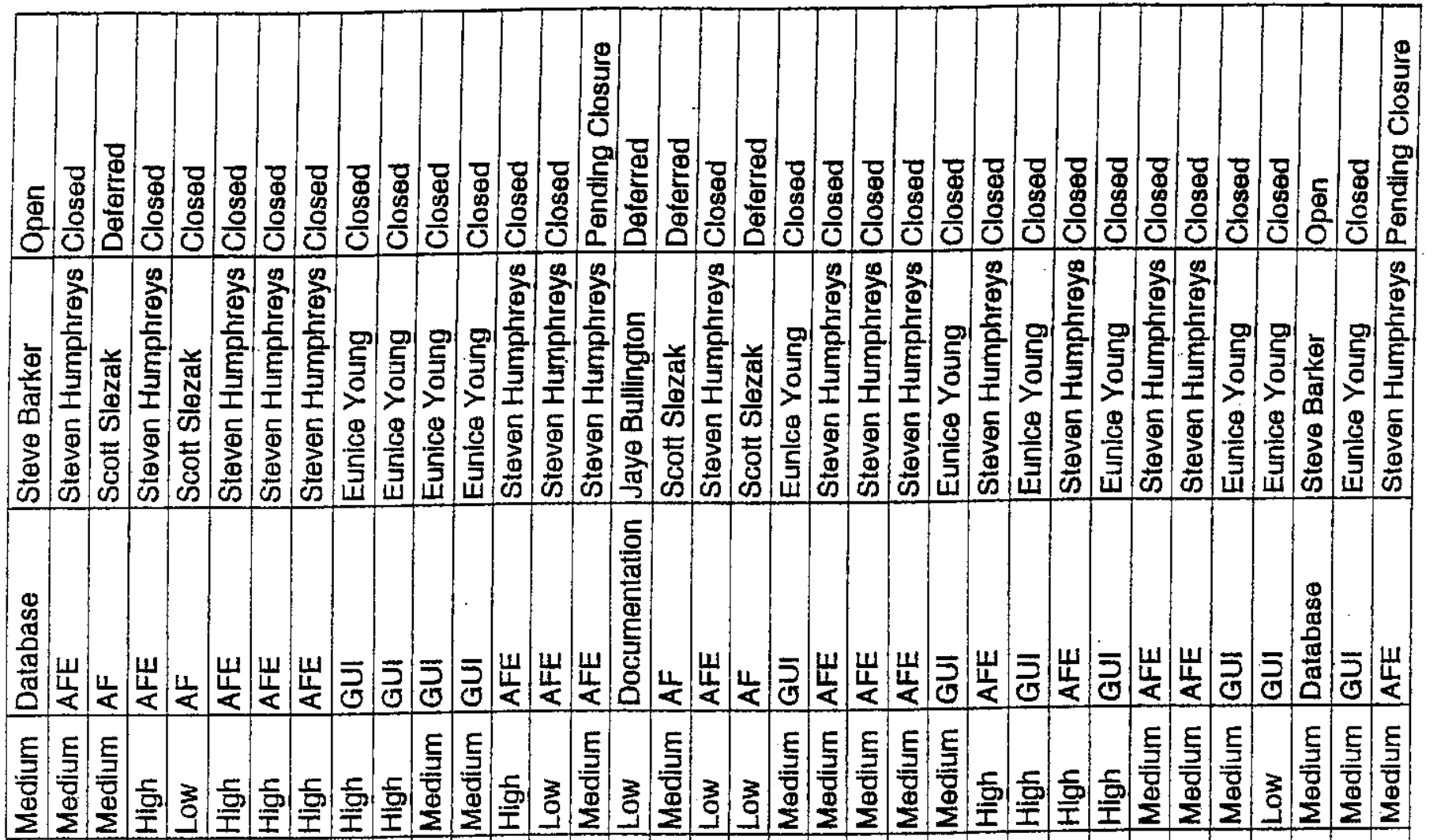

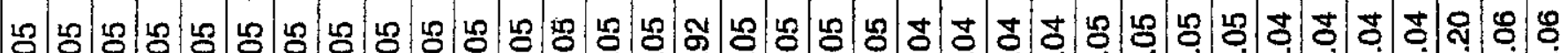

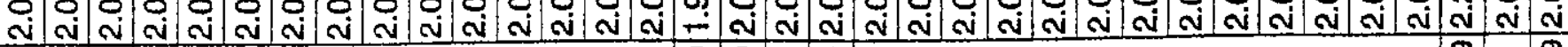

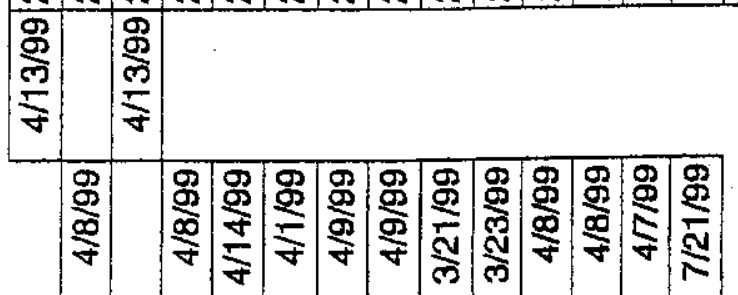

(2)

空

雍

旁䯧
.

$$
\text { 誉 }
$$$$
\text { \& }
$$$$
\text { \$ }
$$

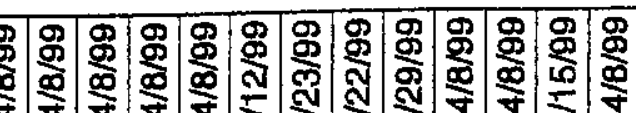

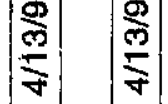$$
\frac{8}{4}
$$

急

\begin{tabular}{|c|c|c|c|c|c|}
\hline & 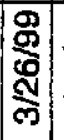 & & $\begin{array}{l}\frac{9}{\mathrm{~g}} \\
\frac{\mathrm{N}}{\mathrm{m}} \\
\end{array}$ & & $\begin{array}{l}\text { \% } \\
\text { 商 }\end{array}$ \\
\hline & $\begin{array}{c}\frac{\partial}{10} \\
\frac{m}{n}\end{array}$ & $\frac{g}{\frac{D}{2}}$ & 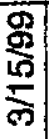 & & $\frac{9}{\frac{9}{6 / 2}}$ \\
\hline & $\begin{array}{l}\infty \\
\vdots \\
0\end{array}$ & 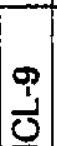 & $\frac{0}{1}$ & & $\frac{N}{0}$ \\
\hline
\end{tabular}

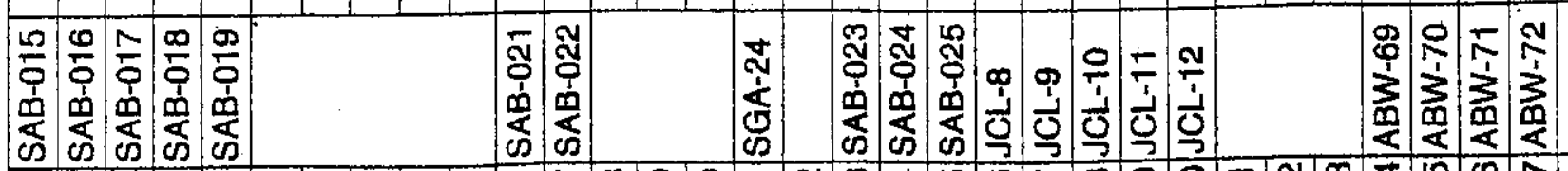

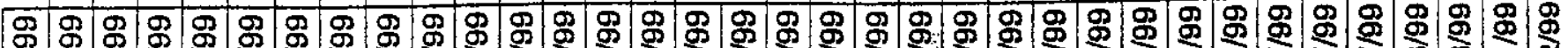

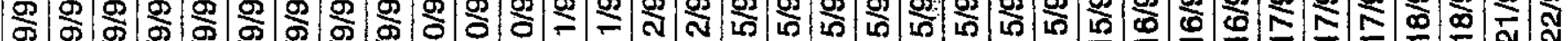

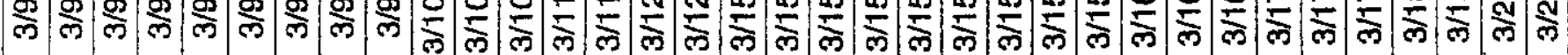

으으. 
山ய山 Ш

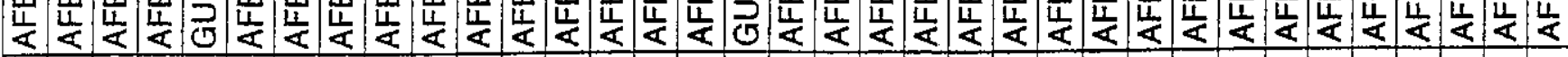

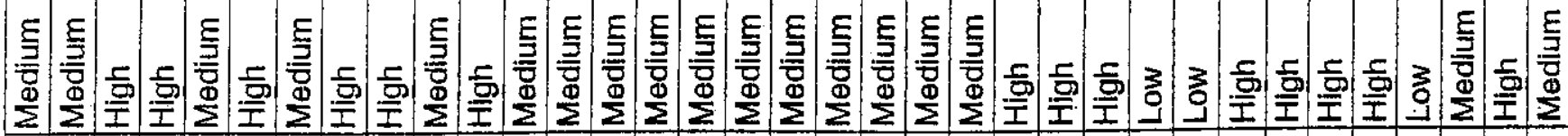

¿

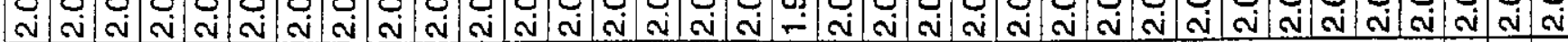

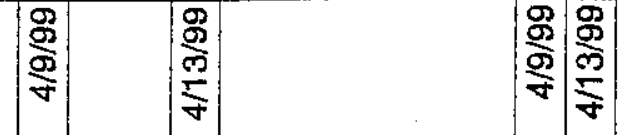

趸

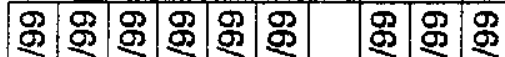
商

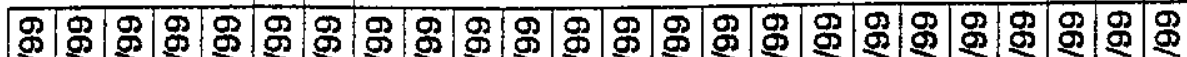
兽 क.

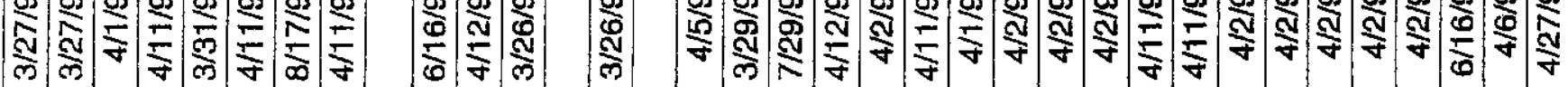
ஜூ

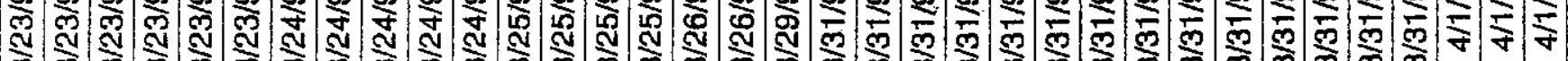

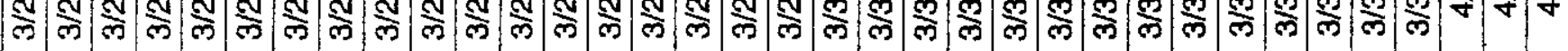

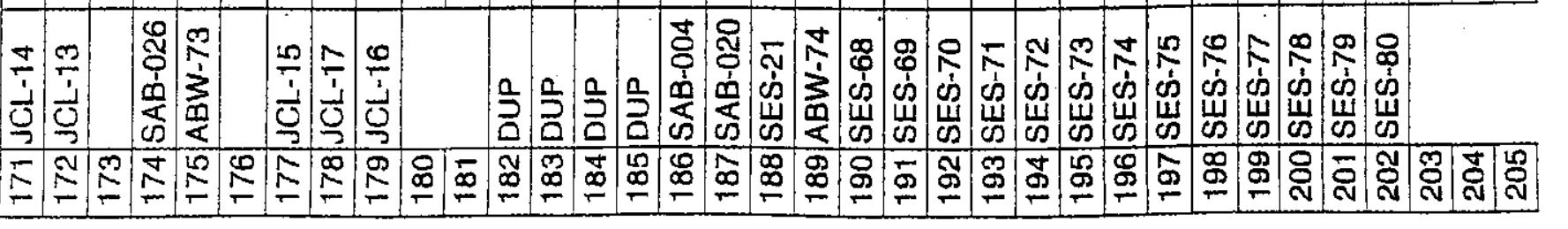




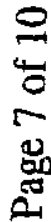

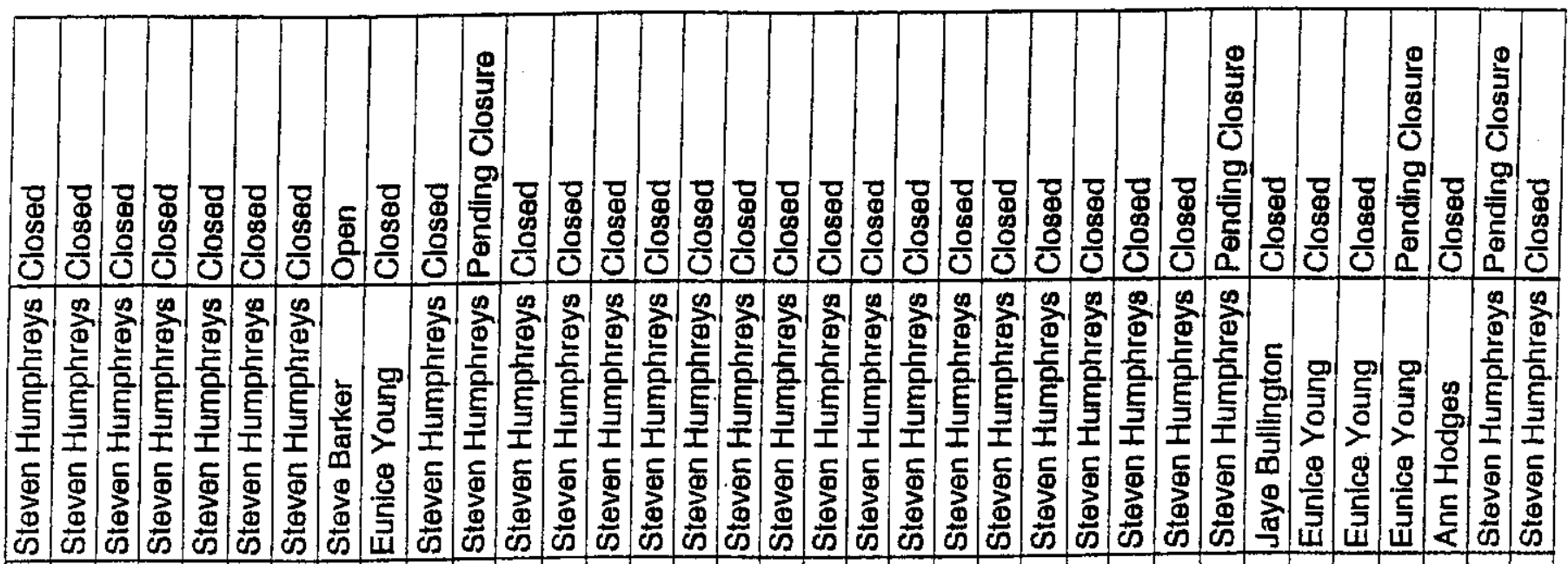

(

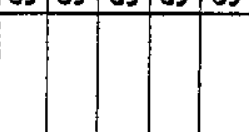

兽

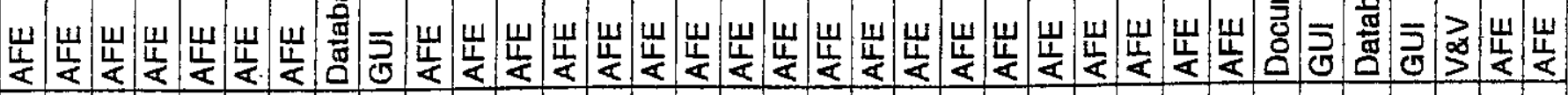

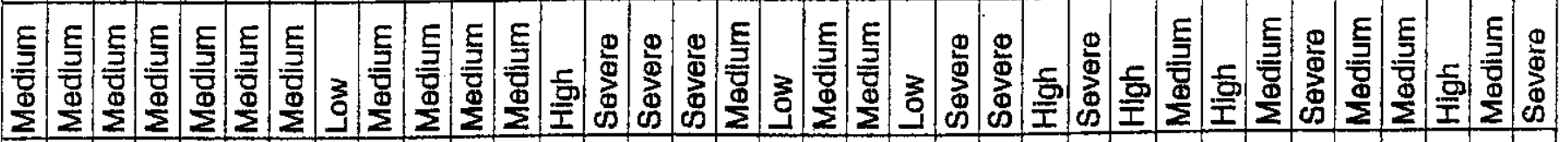

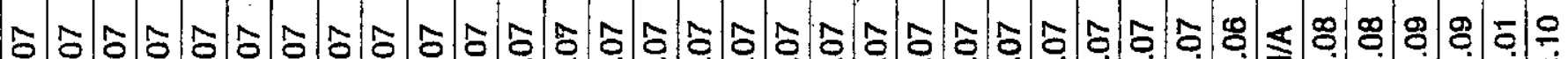

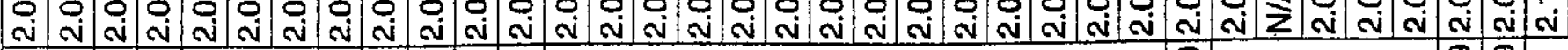

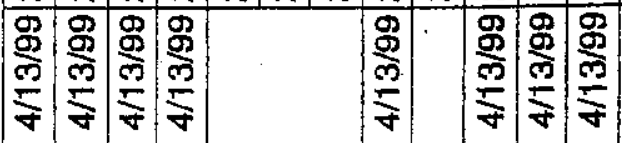
高

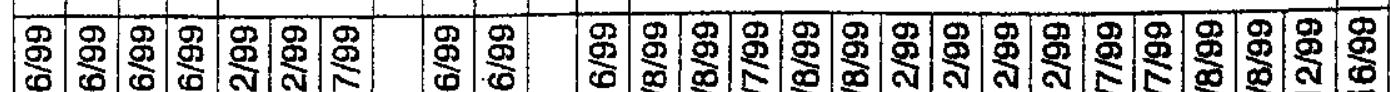

产

gा:

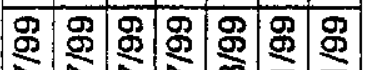

ลิ

\%

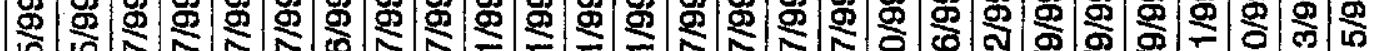

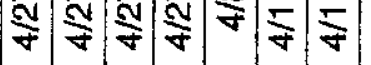

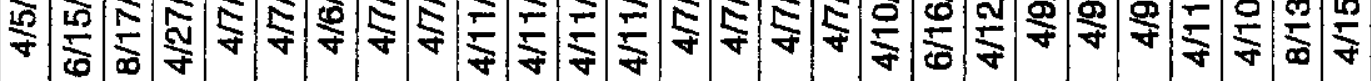

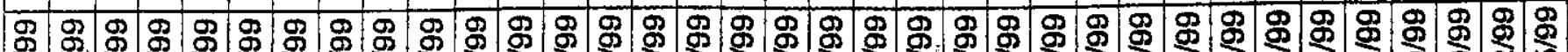

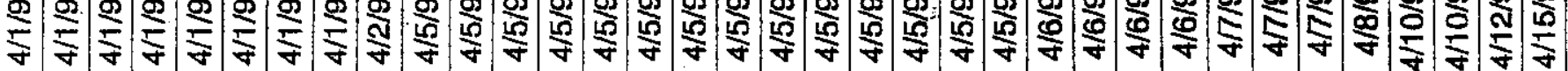




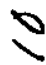

io

$\infty$

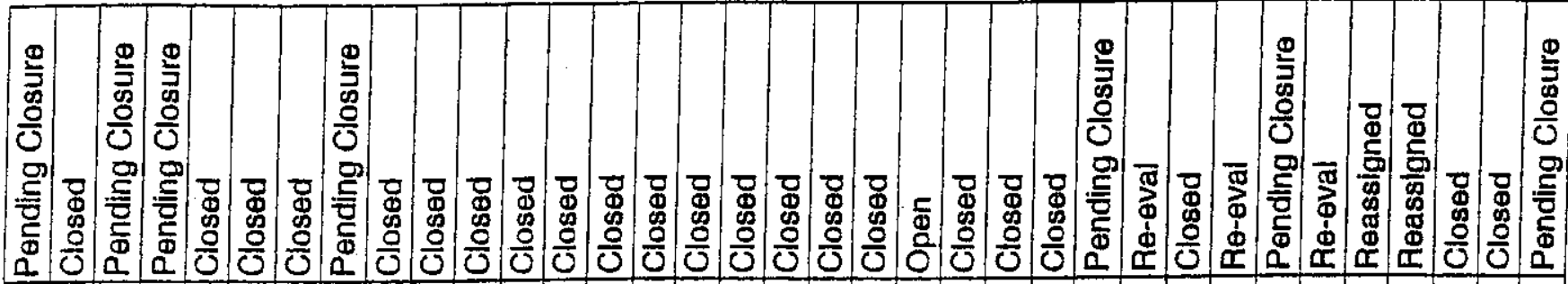

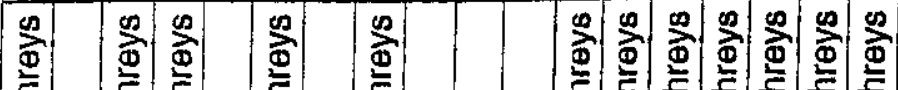

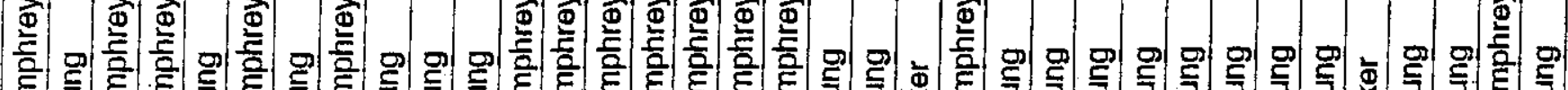

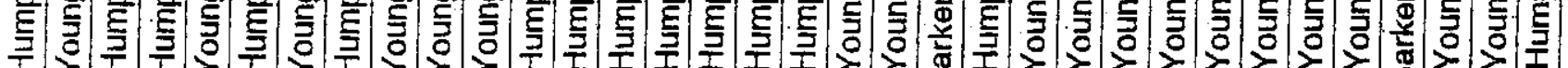

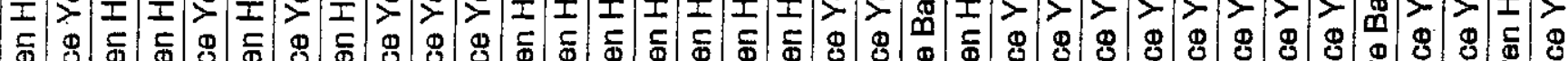

Ф)

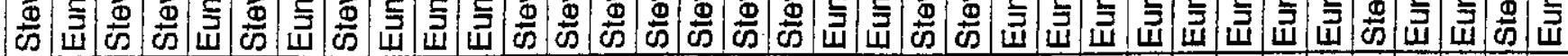

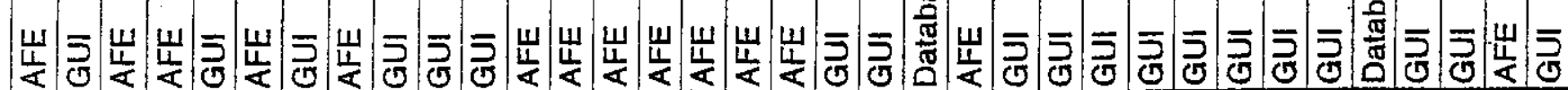

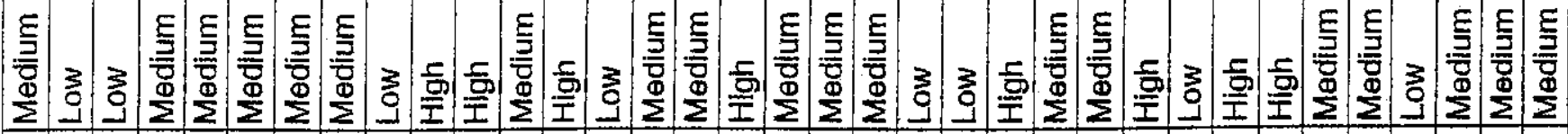

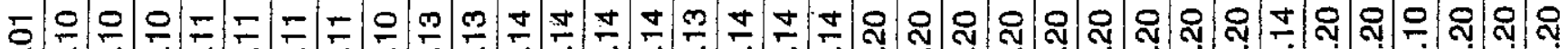

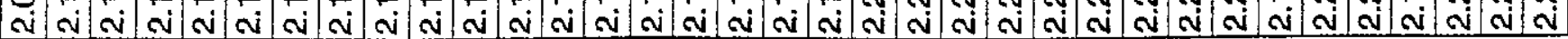
(क)

के

$\sum_{10}$

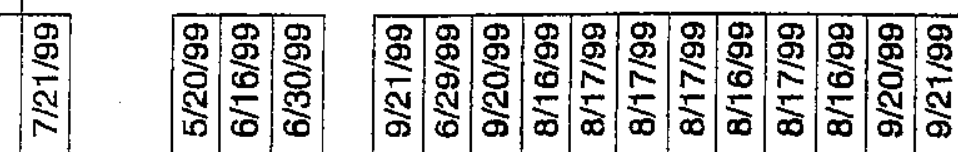

த

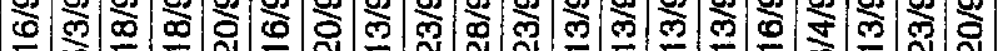

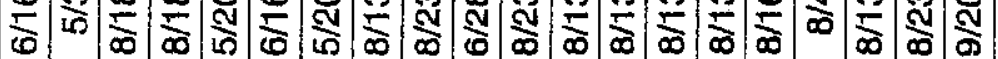

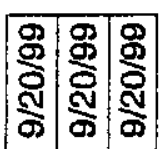

趸

क्: D্ল क के के के के के ने

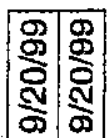

g,

응을

के बे

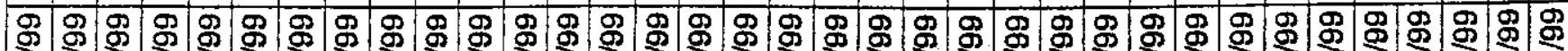

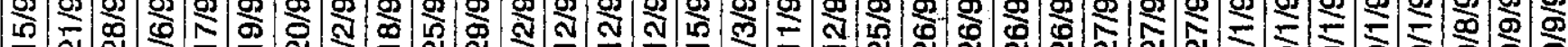

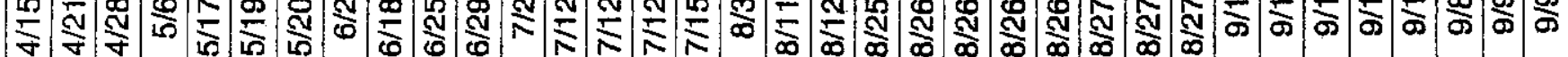

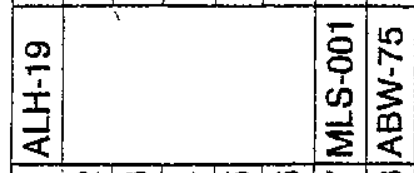

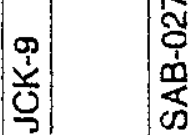

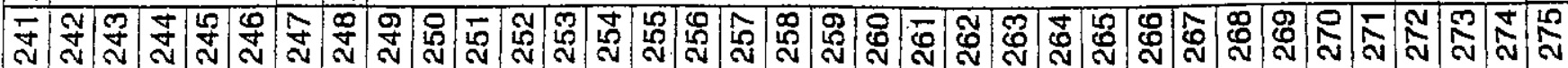




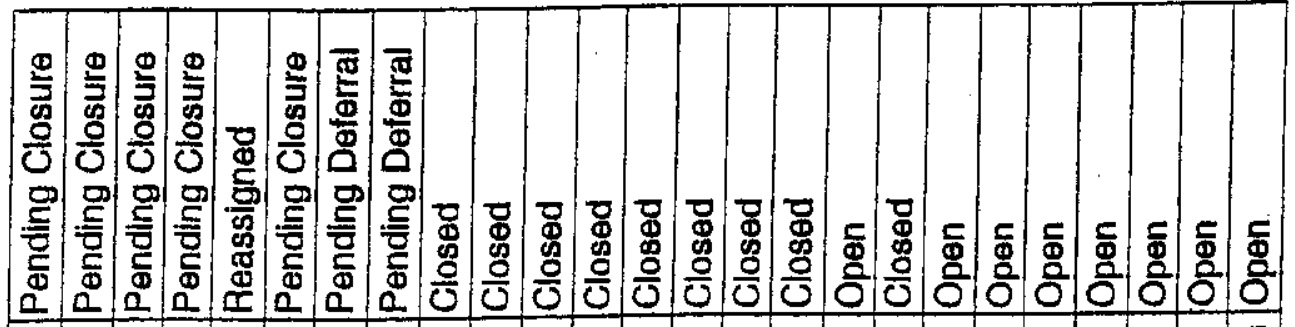

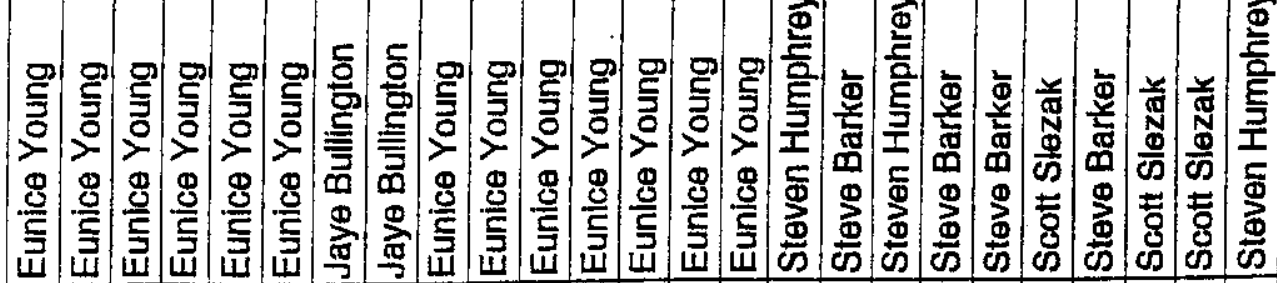

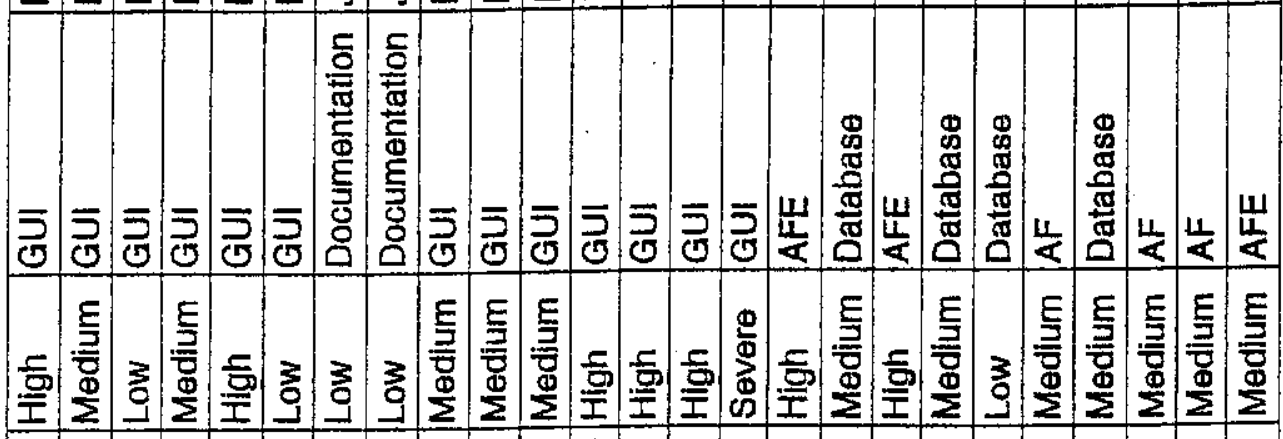

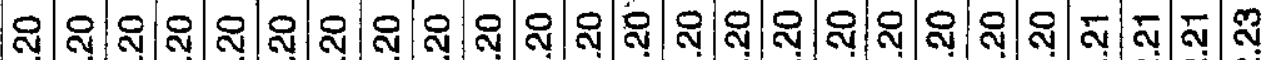

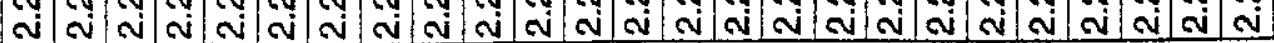


RPP-6888, Rev. 0
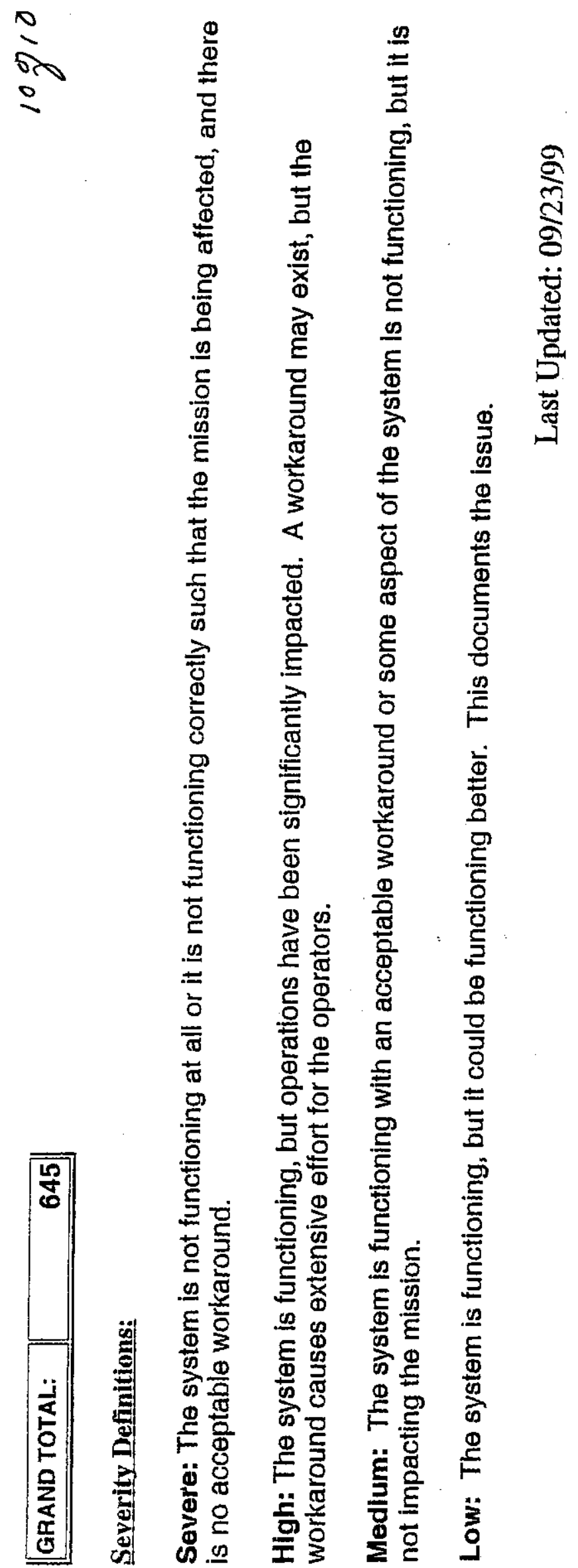
RPP-6888 REV 0

APPENDIX B

DST ACCEPTANCE TEST RESULTS 


\section{RPP-6888 REV 0}

This page intentionally left blank.

Att-B-ii 


\title{
RPP-6888 REV 0
}

\begin{abstract}
APPENDIX B
This Appendix provides the results of the DST acceptance testing. A strict comparison of the benchmark results to the trend analysis results does not consider the magnitude of the difference. For example, if the benchmark mean consequence value is $4.567 \mathrm{E}-05 \mathrm{~Sv}$ and the mean trend test analysis result is $4.566 \mathrm{E}-05$ Sv, a comparison of this type would indicate, that by modifying a specific parameter, the consequences decreased; however, based on the conservatisms and uncertainties incorporated in the Analysis Tool, a better conclusion would be that there is no change. Therefore, to interpret the results, i.e., determine the change in the benchmark values due to changing specific parameters, the comparisons shown in the tables provided for each of the trend test analysis results are based $+/-10 \%$.
\end{abstract}




\section{RPP-6888 REV 0}

This page intentionally left blank.

Att-B-iv 


\begin{tabular}{|c|c|c|c|c|c|c|c|c|}
\hline \multicolumn{3}{|c|}{ DST Aceoptence tant renuth: } & & & & & & \\
\hline \multicolumn{3}{|c|}{\begin{tabular}{|l|l|} 
Seed: 90000000 & \\
\end{tabular}} & & & & & & \\
\hline \multicolumn{9}{|c|}{$\begin{array}{l}\text { Seod: } 90000000 \\
\text { Semple odunt: } 1000 \\
\end{array}$} \\
\hline & & & & & & & & \\
\hline Benounm & $\%$ LRL & & & & $\%$ \%LL & & \%나느 & \\
\hline Tank & & No. wenth & No. dellag. & No. detan. & Median frea. & & Medlan conc. & \\
\hline AN-107. & all aixan & $4223 \mathrm{E}$ & 22|日 & $\mathrm{B} B$ & $3.58 E-09$ & 3 & \begin{tabular}{|l|l|}
$2.38 \mathrm{~B}$ \\
\end{tabular} & \\
\hline & amat & $1220 \mathrm{E}$ & 12 E & 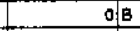 & 1.25E-03: & & $0.251: B$ & \\
\hline & medium & $2009 / \mathrm{B}$ & $B 0 B$ & $8 ; 8$ & 7.a7E-02 & & $2.69 \mathrm{~B}$ & \\
\hline & large & $1000 / \mathrm{B}$ & $0 B$ & 00 & $1.19 \mathrm{E}-0 \mathrm{O}$ & & $10.4 \mid 8$ & \\
\hline & Byoyant diepl & $1229 \mathrm{~B}$ & $18: B$ & 81 8 & $8.00 E+01 E$ & & 2.301 & \\
\hline & Non-bupyant. & 2984 B & 768 & 입 & $1.22 E-03$ & & $2,36 \mid \mathrm{B}$ & \\
\hline$A W=100$ & alf & 11811 & $104 / B$ & 요 & a,36E-04 & & 2.2 .0 & \\
\hline & simalis & 3031 B & 1218 & olB & 4.90E-0.3 & & $0.239 B$ & \\
\hline & modium & $3940 \mid \mathrm{B}$ & $22 / 8$ & OBB & $1.20 E-03$ & & $2.30 \mathrm{~B}$ & \\
\hline & sargye & 304010 & o & $O B$ & $1.69 \mathrm{E}-04 \mathrm{E}$ & & $12.5 \mid \mathrm{B}$ & \\
\hline & Buoyent dinpl & $O B$ & ole & $0 \mathrm{~B}$ & $0.00 E+00$ & & ol & \\
\hline & Non-buoyant & T181: $B$ & $104 B$ & 0.1 & $8.38 E-04$ & & 2.213 & \\
\hline$s Y-103$ & aill sizes & 4030 E & $85 B$ & 10.8 & $5.20 E-00$ & & $3.961 \mathrm{~B}$ & \\
\hline & smail & 1841 E & $14 \mathrm{~B}$ & 0.9 & 7.BSE- $-\infty$ & & $0.775 \mid \mathrm{B}$ & \\
\hline & medlum & $1787 \mathrm{~B}$ & $81 B$ & 1018 & 9.91E- $\infty$ & & 3.836 & \\
\hline & \begin{tabular}{|l|l|} 
large \\
\end{tabular} & $1010: \mathrm{B}$ & O) $\mathrm{B}$ & OB & $1.10 E-03$ & & $20.4 \mathrm{~B}$ & \\
\hline & Buoyent olap & $1614 \mathrm{~B}$ & 3410 & 108 & $7.71 \mathrm{E}+00$ & & $4.25 \mathrm{~B}$ & \\
\hline & Non-buoyant & $3024 \mathrm{E}$ & 911日 & $0 B$ & $1.21 \mathrm{E}-03 \mathrm{E}$ & & 3.75 & \\
\hline sY-101 & sil nizes & 433018 & $506 \mathrm{~B}$ & \begin{tabular}{l|l|}
23 & $B$ \\
\end{tabular} & $3.98 E-03$ & & $6.71 \mathrm{~B}$ & \\
\hline & amalt & 10618 & $38 B$ & 09 & 8.68E-04/E & & $0.242 \mathrm{~B}$ & \\
\hline & medium & 222018 & $\begin{array}{r}+102 B \\
\end{array}$ & 14. $\mathrm{a}$ & $2.54 E+\infty 0 \mid$ & & $7.51 / \mathrm{B}$ & \\
\hline & large & 104018 & $337 \mathrm{~B}$ & 98 & $1.28 E-03$ & & $70.4 / 8$ & \\
\hline & Buoyant diapi & 1336 & ${ }^{85} \mathrm{~B}$ & 2310 & $1.05 E+01 E$ & & $7.35 \mathrm{~B}$ & \\
\hline & Non-buoyant & $2994 \mathrm{~B}$ & $500 \cdot B$ & 의 우 & $1.22 \mathrm{E}-03$ & & $0.04 / \mathrm{B}$ & \\
\hline WIE ANT & & $\mathrm{OE}$ & $0.1 B$ & 018 & $0.00 E+\infty$ & $B$ & o| $\mathrm{B}$ & \\
\hline Inerting-s & $\approx L$ LL & No. ovents & No. deflag. & No. doton. & Medlen treq. & & Medlan cone. & \\
\hline$A N-107$ & all sizes & $4223=$ & $1 \quad 92=$ & \begin{tabular}{|c|} 
s| \\
\end{tabular} & $3.50 \mathrm{E}-\infty \mathrm{s}$ & - & $2.37=$ & \\
\hline AW-108 & all sizes & 11811. & $32<$ & o. & $8.38 E-04$ & $E$ & $2.2=$ & \\
\hline sy-103 & all sizes & $4638=$ & $70<$ & $\Delta<$ & $5.20 E-03$ & 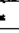 & $3.96=$ & \\
\hline SY.101 & all mizen & $4330=$ & $512<$ & $21 k$ & $3.05 \mathrm{E}-03=$ & $x$ & $8.73=$ & \\
\hline & & & & & & & & \\
\hline Inerting-1 & \%LFL & No. events. & No, deflage & No, deken. & Median freq. & & Median conc. & \\
\hline$A N-107$ & all sizoed & $4223=$ & $92=$ & 日e & $3.50 \mathrm{E}-03$ & $=$ & $2.37=$ & \\
\hline AW.106 & all sizeo & $11811=$ & $32<<$ & 0. & B.36E-04 & $E$ & 2.2. & \\
\hline $9 Y \cdot 103$ & all sizes & 4638: & $94<$ & $11>$ & $5.20 \mathrm{E}-0.3$ & $E$ & $3.96=$ & \\
\hline SY-101 & all sizen & 4330:- & $587=$ & $24>$ & $3.95 E-03$ & 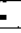 & $6.71=$ & \\
\hline & & & & & & & & \\
\hline Waste vo & d. - \%LFL & No. evente I & No. deflag. & No. deown. & Median freq. & & Median oone. & \\
\hline AN-107 & all sizee & 4352 ; & I $\mathrm{Bo}<<$ & 91> & $4.01 E-0.3$ & 2 & $1.50<$ & \\
\hline & Buoyan! diapl & $1229=$ & 57) & 19. $>$ & $6.08 \mathrm{E}+01$ & $=$ & $5.7>$ & \\
\hline & Non-buoyant: & $2994=$ & $78=$ & 0. & $1.22 \mathrm{E}-\infty)$ & 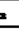 & $4.58 ?$ & \\
\hline$A W-108$ & all sizen & $11788=$ & $60<$ & 4) & 0.90E-04) & 2 & $1.88 \leq$ & \\
\hline & Buoyant diepl & $0=$ & of $=$ & a. - & $0.00 \mathrm{E}+00$ & & 0. & \\
\hline & Non-buoyent & $11871=$ & $104:=$ & o- $=$ & \$.36E-04 & & $3.43>$ & \\
\hline SY-103 & all sizas & $4800>$ & $74<$ & $0 .<$ & 6.38E-03) & & $2.91<$ & \\
\hline & Buoyent diap & $1614=$ & $58>$ & $28 ;$ & $7.71 \mathrm{E}+00$ & & $8.05>$ & \\
\hline & Non-buoyant & $3024=$ & 61: & $0:-$ & $1.21 \mathrm{E}-03$ & 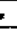 & $5.50>$ & \\
\hline $3 x-101$ & sil sizes & $4582>$ & $245<$ & $.13 k$ & $5.21 \mathrm{E}-03$ & 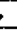 & $4.2 \leq$ & \\
\hline & Quoyent diapi & $1338=$ & $256 .>$ & 943. & $1.05 \mathrm{E}+01$ & $E$ & $29.3>$ & \\
\hline & Non-buoyent. & 2904 & $8301>$ & 야 $x$ & $1.22 E .039$ & $E$ & $18.6>$ & \\
\hline & & & & & & & & \\
\hline Vent: Ra & d. - \%LFL & No. events & No. deflag. & No. delon. & Median treq. & & Median conc. & \\
\hline AN-107 & all sizes & $4223=$ & $1331>$ & $18>$ & \begin{tabular}{|c|}
$3.58 E-09$ \\
\end{tabular} & $E$ & $4.65>$ & \\
\hline & smsall & 1220. & $12=$ & $0 . x$ & $1.25 E-09$ & 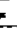 & $0.771>$ & \\
\hline & medium & $2003=$ & $121>$ & $18>$ & $7.07 E+02$ & 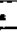 & $4.85>$ & \\
\hline & lafgo & $1000=$ & $0 .=$ & $0=$ & $1.19 E-03$ & 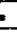 & $20.2>$ & \\
\hline & Buoyant diap & $1229=$ & 57 ? & 19. & B.00E+0t & 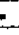 & $5.7 /$ & \\
\hline & Non-buoyent & $2994=$ & $78=$ & $0=$ & 1.22E-03: & 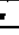 & $4.58 \times$ & \\
\hline$A W=108$ & all & $11811=$ & $104=$ & 0. & 8.36E-04 & 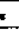 & $3.43>$ & \\
\hline & small & $3231=$ & $12 \mid=$ & $0 .=$ & $4.08 E-09$ & 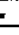 & $0.58 \mathrm{~s}>$ & \\
\hline & medhum & $3900=$ & 92:- & of: $=$ & 1.2BE-03 & $E$ & 3.443 & \\
\hline & large & 3840 & 이 = & of $=$ & $1.68 E-04$ & 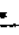 & $15.4 ?$ & \\
\hline & Buoyan! diapl & 0 & $0 .=$ & 이 = & $0.00 E+00$ & 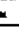 & $0 .=$ & \\
\hline & Non-buoyent: & 11811. & $104:=$ & $0=$ & $8.38 E-04$ & $\approx$ & $3.43 \times$ & \\
\hline SY-103 & all sizeo . . & 4639 & $117 \mid>$ & $26>$ & $3.20 E-03$ & $x$ & $0.39>$ & \\
\hline & small & 18412 & $14 x_{5}$ & $0<<$ & 7. $\triangle 55 E-09$ & $\leq$ & $1.3 \mathrm{~s}$ & \\
\hline & medlum & 1787 , & $103>$ & $28:>$ & $0.91 \mathrm{E}-\infty$ & 2 & $5.5 \mathrm{~K}$ & \\
\hline & largs & 1010 & ofs & $0:<$ & $1.19 E-03$ & $\leq$ & 24.47 & \\
\hline & Buoyant diap & $1614=$ & 56) & $28 />$ & $7.71 \mathrm{E}+\infty$ & $=$ & $0.85>$ & \\
\hline & Non-buoyant & $3024=$ & B1 $=$ & o] & $1.21 \mathrm{E}-\infty$ & $=$ & 5.59 & \\
\hline$s Y-101$ & all atzea & 4330 & 8060 & $94: 3$ & $3.95 E-09$ & 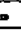 & 21.5 & \\
\hline & smali & 1061. & 38 & 의 $=$ & $8,68 E-04$ & $=$ & 1.973 & \\
\hline & modium & 2220 & 370 & 81 $>$ & $2.34 E+00$ & $E$ & $21,7>$ & \\
\hline & large & $104 \theta$ & $472>$ & $13>$ & $1.2 a E-03$ & 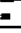 & $24,4]>$ & \\
\hline & Buoyant diap & 1336 & 2563 & $94>$ & $1.05 \mathrm{E}+01$ & $E$ & $29,31>$ & \\
\hline & Non-buoyant & 2994 & $830 ?$ & $0 .=$ & $1.22 \mathrm{E}-0.03$ & $=$ & $80.8>$ & \\
\hline & & & & & & & & \\
\hline
\end{tabular}




\begin{tabular}{|c|c|c|c|c|c|c|c|c|c|c|}
\hline \multicolumn{2}{|c|}{ Vent, Ravarinc. \% \% LFt. } & \multirow{2}{*}{\begin{tabular}{|l|} 
No, events \\
4223 \\
\end{tabular}} & \multirow{2}{*}{ 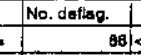 } & & No, delon. & Modian freq. & & Medten conc. & & \\
\hline AN-107 & Ell akzes & & & & $2<$ & $\quad 3.58 E-03$. & & $\mid \begin{array}{ll}\mid & 0.548 \\
\end{array}$ & & \\
\hline & smail . & $|\ldots 1220|=$ & $-\ldots 12$ & & 이- & $1.25 \mathrm{E}-03=$ & & $0.033<$ & & \\
\hline & medium & $2003=$ & 74 & & $2 \leqslant$ & $7.87 \mathrm{E}-02 \mid=$ & & $0.562<<$ & $\leq$ & \\
\hline & itargo & $1000:=$ & o. & $=$ & $0=$ & $1.19 \mathrm{E}-03$ & & $10.7<$ & & \\
\hline & Bucyant disp & 1229 & $10<$ & & $2 \mid \leq$ & $6,00 E+0 ;=$ & & $\left.0.447\right|_{K}$ & & \\
\hline & Non-buoyant & $2994=$ & 78 & & 0.0 & $1.22 \mathrm{E}-03=$ & & $0.805<<$ & & \\
\hline AW-106 & All & $\pm 1811=$ & 104. & & $0 .-$ & 9.3日E-04 = & & $0.705<$ & & \\
\hline & amall & $3831=$ & 12 & & 요 = & $4.90 \mathrm{E}-\infty)=$ & & $0.02811<$ & & \\
\hline & madium & $3940=$ & $92=$ & & $0,=$ & $1.28 \mathrm{E}-\infty$ & & $0.823 \mid<$ & & \\
\hline & large. & 3940 & ㅇ․ & $=$ & 이- & $1.60 E-04$ & & $9.42<$ & & \\
\hline & Buoysnt diap & o| & o: & $=$ & 0.0 & $0.00 \mathrm{E}+\infty 0$ & & $0=$ & & \\
\hline & Non-buoyant & $11811=$ & $104=$ & & $0=$ & $0.36 \mathrm{E}-04=$ & & 0.705 < & & \\
\hline $5 Y-103$ & ali sizes & $4838=$ & $74<$ & & 1.5 & $5.20 \mathrm{E} .00 \mathrm{a}-$ & & $0.857 \mid<$ & & \\
\hline & amall & $1841 />$ & $14 k$ & & 이 & $7.05 E-\left.\infty\right|_{<}$ & & $0.247 \mid<$ & & \\
\hline & Imedium & $1787 \mid>$ & $601>$ & & 1) & $9.9 \div E-03>$ & & $\left.1.18\right|_{k}$ & & \\
\hline & Large & $1010 \mid<$ & $0<$ & & $0 \mid<$ & $1.10 \mathrm{E}+03<<$ & & $15.4>$ & & \\
\hline & Buoyent diap? & $1614=$ & $13<$ & & $1<$ & $7.71 \mathrm{E}+\infty 0$ & & $0.761<$ & & \\
\hline & Non-buoyant & $3024=$ & 81! & & $0=$ & $1.21 E-\infty)=$ & & $1.3 \mathrm{~K}$ & & \\
\hline $5 Y \cdot 101$ & all stzes & $4330=$ & $368 \mid<$ & & $2,<$ & $3.06 E-03$ - & & $1.111 \leq$ & & \\
\hline & small & $1001=$ & 36. & & o.m & $0.68 \mathrm{E}-04=$ & & $0.0258<\mathrm{K}$ & & \\
\hline & medium & $2220=$ & 184.5 & & $2 \mid c$ & $2.54 E+00 \mid=$ & & $1.07 \mid<$ & & \\
\hline & larga & $1049=$ & $148<$ & & of & $1.28 E-03 \mid=$ & \pm & $20.1<$ & & \\
\hline & Buoyant dispo & $1338=$ & $56 .<$ & & $2<$ & $1.05 E+01 \pi$ & $\pi$ & $1.00 \mid<$ & & \\
\hline & Non-bucyant & $2994=$ & $312 / 5$ & & $0 .=$ & $1.22 E-03=$ & 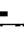 & $\left.1.16\right|_{\mathrm{S}}$ & & \\
\hline & & & & & $\ldots$ & & & 1 & & \\
\hline Intrussive ops & 1. All • ino & No. events & No. dollag. & & No. deton. & Median freq. & & Median conc. & & \\
\hline$A N-107$ & at aizea & $4223=$ & $\mathbf{9 2}=$ & & $81=$ & | 3.50E-03/- & & $2.36--$ & & \\
\hline & smail & $1220=$ & $12=$ & & $0=$ & $1.25 E-03 \mid=$ & & $0.251=$ & & \\
\hline & medium & $2003=$ & $801=$ & & $a=$ & $1.57 \mathrm{E}-\left.\infty 2\right|_{\mathrm{K}}$ & & $2.60 /=$ & & \\
\hline & Large & $1000=$ & $0 \mid=$ & & $0=$ & $1.18 \mathrm{E}-0 \mathrm{0}=$ & & $18.4 .=$ & & \\
\hline & Buoyant displ & $1229=$ & 18) $=$ & & 8. $=$ & $6.03 E+01=$ & & $2.33 \mid=$ & & \\
\hline & Non-buoyani & $2994 \mid=$ & 78 & & 의= & $1.22 E-03=$ & & $2.381=$ & & \\
\hline AW-106 & al! & $12004 \mid>$ & $\theta 0<$ & & 4) $>$ & $5.54 \mathrm{E}-04<<$ & & $2.231>$ & & \\
\hline & small & 3898 & $8<$ & & $4>$ & $3.59 \mathrm{E}-03 \mathrm{0} \mid<$ & & $0.234<$ & & \\
\hline & modum & $4004>$ & $30<$ & & ㅇ- & $9.31 \mathrm{E}-04<$ & & $2.37=$ & & \\
\hline & Large & $4004>$ & 0 & $\square$ & of $=$ & $9.53 \mathrm{E} .05$ & & $12.9 \%$ & & \\
\hline & Buoynnt diap| & 0. & 0. & & $0=$ & $0.00 E+00=$ & & $0 \mid=$ & & \\
\hline & Non-buoyant & $12004 />$ & $88 \mid<$ & & . $>$ & $3.54 E-04<$ & & $2.231>$ & & \\
\hline$s Y \cdot 103$ & all sizes & $4574<$ & $93 \mid<$ & & $13>$ & $4.08 E-03<$ & & $4.17 \mid>$ & & \\
\hline & 8 mall & $1817>$ & 186 & & $0<$ & $7.60 \mathrm{E}-03 \leq$ & & $0.774 \mid<$ & & \\
\hline & medium & $\nmid 754>$ & $\left.n\right|_{3}$ & & 13. $>$ & 9.83E-03,> & & $3.97<$ & & \\
\hline & large & $1000 \mid<$ & of & $<$ & $0 \mid<$ & $1.15 E-03<<$ & & $20.5>$ & & \\
\hline & Buoyant disp & $1588 \mid<$ & 27.5. & & $13 \mid>$ & $8.05 E+001>$ & & 4.54l> & & \\
\hline & Nen-buoyant & 3005 & $68 ;$ & $\geq$ & 이 $=$ & $1.19 \mathrm{E}-93<$ & & $3.07 />$ & & \\
\hline sy-101 & atil sizes & $4320=$ & $572>$ & $\geq$ & $281>$ & $3.86 \mathrm{E}-0.03<$ & & $\begin{array}{l}6.75 .2 \\
\end{array}$ & & \\
\hline & small & 1055.4 & 45:2 & & $0=$ & $7.90 \mathrm{E}-04 \mathrm{~K}$ & & $0.247>$ & & \\
\hline & modium & $2214=$ & $174 / 5$ & & $21>$ & $3.17 E+00>$ & & $7.41<$ & & \\
\hline & Tharge. & 1051 - & 353/, & & 8. $<$ & $1.31 \mathrm{E}-003>$ & & $72.7>$ & & \\
\hline & Buoyant diapl & $1323=$ & $57 \mid<$ & $\leqslant$ & $29)>$ & $1.07 E+01>$ & & 7.4813 & & \\
\hline & Non-buoyam & 2997 : & $515>$ & $\geq$ & $0=$ & $1.29 \mathrm{E}-03=$ & & $5.85 \mid<$ & & \\
\hline & & & & & 1 & & & 1 & & \\
\hline Intrusive ope & . Nan - red & No. events & No, daflag. & & No. deron. & Medkan freq. & & Median conc. & & \\
\hline AN-107 & ail sizes & 4223 & 92 & & $8=$ & $3.50 E_{-1}-03 .=$ & & $2.38=$ & & \\
\hline & smail & 1220 - & $12=$ & & $0 .=$ & $1.25 E-03:=$ & & 0.251 J & & \\
\hline & medium & $2003=$ & $80=$ & & $8=$ & $7.87 \mathrm{E}-02$ & & $2.60=$ & & \\
\hline & targo & $1000=$ & 0. & & $0=$ & $1.19 \mathrm{E}-001=$ & & $18.4 \mathrm{a}$ & & \\
\hline & Buoyant diap| & $1229=$ & 16 & & $8=$ & $B .09 E+01=$ & & 2.33. & & \\
\hline & Non-buayant & $2994=$ & 76. & 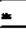 & $0=$ & $1.22 \mathrm{E}-00=$ & & $2.38=$ & & \\
\hline$A W-106$ & all & $\left.17811\right|_{=}$ & $104 \mid=$ & & 우 $=$ & 9.38E-04 = & & $2.2=$ & & \\
\hline & smasl & $3931=$ & 12 & & 요 & $4.886-03=$ & & $0.2391=$ & & \\
\hline & medium & 3940 - & $\theta 2=$ & & 이 = & $1.20 E-103=$ & & 2.38 .2 & & \\
\hline & Jlarge & $3840=$ & $0:=$ & $=$ & $0 .=$ & 1.68E-041- & & $12.81=$ & & \\
\hline & Buoyant diop & 이드. & of & 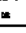 & 0. & $0.00 \mathrm{E}+\infty \mathrm{O}=$ & & of & & \\
\hline & Non-buoyant & $11011=$ & $104:=$ & $m$ & $0=$ & $8.36 \mathrm{E}-04=$ & & 2.2. & & \\
\hline$S Y-103$ & all sizes & $4638=$ & 95 & 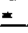 & $10=$ & $5.20 \mathrm{E}-00 \mathrm{I}$ & & $3.96=$ & & \\
\hline & smail & $1847>$ & $14 .<$ & & $0 \mid<$ & $7.85 E-03<$ & & $0.775<$ & & \\
\hline & medium & $1787 \%$ & ot? & & 1012 & $9.91 \varepsilon-03,>$ & & $3.93<$ & & \\
\hline & large & $1010<$ & 0 & & 0.5 & $1,19 E-\infty 3 \mid \leq$ & & 20.43 & & \\
\hline & Buoyant diapl & $1614=$ & $34=$ & & $10=$ & $7.71 E+\infty 0 \mid=$ & & $4.25=$ & & \\
\hline & Non-byoyant & 30241. & 81. & & 0.1 & $1.21 \mathrm{E}-\infty 3=$ & & $3.75=$ & $=$ & \\
\hline$S Y=101$ & fall aizea & 4330 & $5655=$ & & $23=$ & $3.95 E-\infty)=$ & & 8.71 0 & \pm & \\
\hline & Small & $10811=$ & $36]=$ & & $0=$ & $8.68 \mathrm{E}-04$ & & 0.242 & $=$ & \\
\hline & medbum & $2220 \mid=$ & 192. & & $11 .=$ & $2.54 E+00=a$ & & $7.51=$ & & \\
\hline & large & $1049 \mid=$ & 337. & $=$ & $9 \mid=$ & $1.28 E-03 \mid-$ & & $70.4=$ & $=$ & \\
\hline & Buoyant dispe & 13966 & 255: & $=$ & 23 - $=$ & $1.05 E+01 \mid=$ & & $7,35$. & 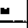 & \\
\hline & Non-buoyant & $2.994 K_{\mathrm{S}}$ & 500:- & & $0=$ & $1.22 \mathrm{E}-\infty 3=$ & & $8.04=$ & & \\
\hline & & & & & & & & & & \\
\hline & & & & & & & & & & \\
\hline & & & & & & & & & & \\
\hline & & & & & & & & & & \\
\hline & & & & & & & & & & \\
\hline
\end{tabular}




\begin{tabular}{|c|c|c|c|c|c|c|c|c|c|}
\hline \multirow{7}{*}{\begin{tabular}{|l} 
Intruasive of \\
AN-107 \\
\end{tabular}} & Non - inc & Na. ovents & No. defleg. & No. daten, & Median trea. & & Moden cone. & & \\
\hline & all azes & $4223=$ & .92 $=$ & B & 3.50E-00 & \pm & 2.36 & & \\
\hline & errall & $12201=$ & $12=$ & 이 & $1.25 E-035$ & $=$ & $0,251=$ & & \\
\hline & medium & $20031=$ & $80=$ & s. & $7.07 E-02$ & 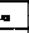 & $268=$ & & \\
\hline & terge & $1000=$ & $0=$ & a. & $1.19 E-\infty 3$ & $E$ & $18.4=$ & & \\
\hline & Buoyant dtap & $12291=$ & $161=$ & aid. & $0,085+01$ & \pm & $2.33=$ & & \\
\hline & Nan-buoyant & $2894=$ & 7810 & of & $1.22 E-\infty$ & $\approx$ & $2.39=$ & & \\
\hline \multirow[t]{6}{*}{$A W-10 B$} & $\mathrm{aH}_{\mathrm{H}}$ & $11811=$ & $104=$ & of & 6.3eE-04 & $=$ & $22 \pi$ & & \\
\hline & omall & $3031:=$ & $12=$ & of & 4.98E-09: & \pm & 0.230. & & \\
\hline & medium & $3940=$ & $92=$ & $0=$ & $1.28 E-\infty 3$ & 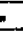 & $2.38=$ & & \\
\hline & largen & $3840=$ & 이․ & $0=$ & 1.60 E.04 & $E$ & $12.6=$ & & \\
\hline & Buoyant diappl & of & 0. & of & $0.00 E+\infty$ & & 0. & & \\
\hline & Non-buoyant & 170111= & 100 & $0=$ & 0.36E-04: & 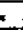 & $2.2 .=$ & & \\
\hline \multirow[t]{6}{*}{ SY-10s } & Alf oizen & 4638 & $95=$ & $10=$ & $5.20=-03$ & $E$ & $3.96=$ & & \\
\hline & ame日 & 104t $>$ & $14 \mathrm{~S}_{\mathrm{S}}$ & 0.5 & $7.055-03$ & $\leq$ & $0.775<<$ & & \\
\hline & medlum & 1787 > & 815 & 101? & 8.91E-03 & 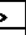 & $3.231<$ & & \\
\hline & Large & $1010<$ & $0: 5$ & of & $1.10 \mathrm{E}-\infty \mathrm{a}$ & $\leq$ & 20.47 & & \\
\hline & Buoyani diapi & $1814 .=$ & $34=$ & $10=$ & $7.71 E+\infty$ & & 4.25 .6 & & \\
\hline & Non-bwoyent: & $3024=$ & 61. & of & $1.21 E-03$ & 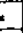 & 3.76 . & & \\
\hline \multirow[t]{7}{*}{ SY-101 } & ald sizes & $4330 \mid-$ & 5es: : & $23=$ & $3.95 E-03$ & & $6.71=$ & & \\
\hline & amsall & $1067=$ & 36 & $0=$ & $8,6 B E-04$ & & $0.242=$ & & \\
\hline & medium & $2220 \mid=$ & $182=$ & 14:- & $2.54 \mathrm{E}+00$ & & $7.511=$ & & \\
\hline & terga & $7040=$ & $337=$ & $9=$ & $1.28 E-03$ & & $70.4=$ & & \\
\hline & Bucyant diaps & 1336 - & $65=$ & 23 & $1.05 E+01$ & $x$ & $7.35 \mid=$ & & \\
\hline & Non-buoyant & $294 / 5$ & $500=$ & $0]$ & $1,22 \mathrm{E}-00$ & . & $8.06-$ & & \\
\hline & & & & & & & & & \\
\hline \multicolumn{2}{|c|}{ Intrueve opa Localtrad } & No. ovente & No. deflueg. & No. daton. & Median frea. & & Median conc. & & \\
\hline AN-107 & it sizes & $4223=$ & $92=$ & $\mathrm{g}-$ & $3.68 \mathrm{E}-03=$ & & 2.38 & & \\
\hline & small & $1220=$ & $12=$ & 0. & $1.26 E-09=$ & & $0.251=$ & & \\
\hline & medium & $2003=$ & $801=$ & $8=$ & $7.97 \mathrm{E}-2.2$ & & $2.88=$ & & \\
\hline & large & $1000=$ & of $=$ & 0. & $1.39 E-03$ & & $18.4=$ & & \\
\hline & Bupyant displ & $1229=$ & $16=$ & ox & $8.08 E+01$ & & $2.33=$ & & \\
\hline & Non-buoyent & $2804=$ & $76=$ & $0=$ & $1.228-03$ & & $2.38 \mid=$ & & \\
\hline AW-108 & all & $12003 />$ & $100>$ & $0=$ & B.61E-O4) & & $2.23 \mid>$ & & \\
\hline & small & 3995 & $12=$ & 0.1- & 4. $25 E-03$ & & $0.232 /<$ & & \\
\hline & modium & $4004>$ & $96>$ & $0 .=$ & $1,44 E-03$ & & $2.44>$ & & \\
\hline & larges & $40041>$ & 0 & ole & 1.02E-04/ & & $13>$ & & \\
\hline & Buoyent diep & $0:=$ & 의= & $0=$ & $0.00 E+00$ & & ole & & \\
\hline & Non-buoyant & $12009 />$ & $100>$ & 의 & $8.01 \mathrm{e}-04 \mathrm{~s}$ & & 2.23 & & \\
\hline SY-103 & It sikes & $4603:=$ & $115>$ & $7 \mathrm{~s}$ & 5.09E.03 & $\varepsilon$ & $3.85=$ & & \\
\hline & amall & $1828>$ & 24 & 015 & $7.69 \mathrm{E}-\infty$ & $\leq$ & $0.768<$ & & \\
\hline & modkum & 1772 , > & 21 ? & $7 x$ & $9.78 \mathrm{E}-\infty$ & & $3.89<$ & & \\
\hline & iarge & $1005 /<$ & $0 \mid<$ & 0,5 & $1.20 E-09$ & & $20.3 \mid>$ & & \\
\hline & Buoyant datapi & 1594 < & $48>$ & 75 & $7.85 \mathrm{E}+00$ & 2 & $4.27=$ & & \\
\hline & Non-buoyant & $3009=$ & $67=$ & 0 & $1.20 \mathrm{E}-03$ & & 3.027 & & \\
\hline $3 Y-101$ & all aizes & $4330=$ & 505 & $23=$ & $3.95 \mathrm{E}-03$ & & \begin{tabular}{c|c}
6.71 & $=$ \\
\end{tabular} & & \\
\hline & emrall & $1067=$ & 36. & $0=$ & 4.6BE-04 & & $0.242=$ & & \\
\hline & medium & $2020=$ & $102=$ & $34=$ & $2.54 E+00$ & & $7.51=$ & & \\
\hline & large & $1040=$ & $337=$ & 1) & 1.28E-09: & & $70.4=$ & & \\
\hline & Buoyent diepl & $1336=$ & $85=$ & $23=$ & $1.05 E+01$ & & $7.35=$ & & \\
\hline & Non-buoyant & $2994=$ & $500=$ & $0=$ & $1.22 \mathrm{E}-\infty$ & 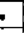 & $6.04=$ & & \\
\hline & & & & & & & & & \\
\hline Intruative of & Locatinc & No. events & No. doffiag. & No. daton. & Median treq. & & Median cona. & & \\
\hline $\mathrm{AN}=107$ & sil sires & $4223=$ & $92-$ & 8- & $3.58 \mathrm{E}-00$ & 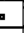 & $2.36=$ & & \\
\hline & armell & $1220=$ & 12 & . & $1.25 \mathrm{E}-\infty$ & 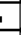 & $0.251=$ & & \\
\hline & medium & $2003=$ & 80 & 禁 & $7.87 \mathrm{E}-02$ & & $2.68=$ & & \\
\hline & largo & $1000=$ & o: & of & $1.18=00$ & & $18,4=$ & & \\
\hline & Buoyant dispi & $1229=$ & 161. & 8.:- & $8.00 \mathrm{E}+01$ & & $2.33=$ & & \\
\hline & Non-bwoyant & $28001=$ & 76 & 0. & $122 \mathrm{E}-03$ & & 2.39 & & \\
\hline AW-108 & alt & $11920 \mid=$ & 100 s & of & $3.805-04$ & & $2,10=$ & & \\
\hline & email & $3868=$ & ats & 이 & $4.90 \mathrm{E}-\infty$ & & $0.230=$ & & \\
\hline & medium & $3976 \mid=$ & $92:=$ & 의: & 1.32E- $\infty$ & & $2.31<$ & & \\
\hline & largo & 3978 & $0=$ & of & $1.58 \mathrm{E} \cdot 04$ & & $12.8 \mid>$ & & \\
\hline & Buoyant dispp & $0=$ & 0. & a. & $0.00 E+00$ & & $0=$ & & \\
\hline & Non-buoyant & $11020=$ & $+00<5$ & of & $7.90 \mathrm{E}-0 \mathrm{~A}$ & & $2.18 \mid=$ & & \\
\hline $5 Y-103$ & al sizes & $4638=$ & $85=$ & $10=$ & $5.20 \mathrm{E}-03$ & & 3.96 & & \\
\hline & smail & $1841>$ & 145 & of & $7.25 \mathrm{E}-03$ & & $0.775<$ & & \\
\hline & medium & $17 a 7>$ & 813 & 10 & $8.81 E-03$ & & $3.93<$ & & \\
\hline & large & $1010 \mid<$ & 0.8 & of & $1.19 \mathrm{E}-0 \mathrm{~g}$ & $\leq$ & $20.4>$ & & \\
\hline & Buoyant diap & $1814=$ & 34. & 10. & $7.70 E+00$ & & $4.25 \mid=$ & & \\
\hline & Non-buoyent & $3024=$ & B1. & o. & $1.21 \mathrm{E}-00$ & $=$ & 3.75 - & & \\
\hline$\$ Y .101$ & old sizes & $4330=$ & 585 . & $23:$ & 3.96E.03: & 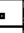 & $6.71=$ & & \\
\hline & matl & $10811=$ & 36 & of & $8.68 \mathrm{E}-04$ & & $0.242=$ & & \\
\hline & modium & $22201=$ & 192 & 14. & $2 . \$ 16+\infty 0$ & & $7.51=$ & & \\
\hline & large & 1049 & 337 & o. & $1.28 E-\infty$ & & $70.4=$ & & \\
\hline & Bucyant diop & 1936 & 68 & $23=$ & $1.05 E+01$ & & $7.35=$ & & \\
\hline & Non-buoyent & $2094]$ & 500 & of. & $1.22 \mathrm{E}-\infty$ & & $0.04=$ & & \\
\hline & & & & & & & & & \\
\hline
\end{tabular}




\begin{tabular}{|c|c|c|c|c|c|c|c|c|}
\hline \multicolumn{2}{|c|}{ Intruaive ops Globelited } & \multirow{2}{*}{\begin{tabular}{|c|} 
No. eventin \\
4223 \\
\end{tabular}} & \multirow{2}{*}{$=\mid$ No. defleg. } & \multirow{2}{*}{$=$} & \multirow{2}{*}{$\begin{array}{l}\text { Median freq. } \\
\\
\end{array}$} & \multirow{2}{*}{\multicolumn{2}{|c|}{$\begin{array}{r}\text { Medtari cone. } \\
2.3 \theta-\end{array}$}} & \\
\hline AN-107 & aill aizes & & & & & & & \\
\hline & armail & $1220=$ & $|12|=$ & $0=$ & - $\quad 1,25 E-03 .=$ & $0.251=$ & & \\
\hline & medium & $20031=$ & $80:=$ & $\theta: \mathrm{x}$ & $3.94 \mathrm{E}-02 \mid<$ & $2.68-=$ & & \\
\hline & targe & $1000=$ & $0=$ & 010 & $1.18 \mathrm{E}-0 \mathrm{O}=$ & $16.4 \ldots$ & & \\
\hline & Butoyant disp & $1229 .=$ & $16=$ & $8=$. & $6.11 E+01=$ & $2.33 .=$ & & \\
\hline & Non-buoyant & $2994=$ & $76=$ & 0.0 & $1.22 \mathrm{E}-03 \mathrm{H}=$ & $2.3 s:=$ & & \\
\hline AW-106 & all & $11885=$ & $72 \times$ & 4) $>$ & $1.18 E-03 / 3$ & $2.2=$ & & \\
\hline & amell & $3857=$ & $4 k$ & 4) & $6.81 \mathrm{E}-\infty)>$ & $0.251>$ & 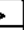 & \\
\hline & medium & $3984=$ & $80<$ & $0=$ & $2.00 E-03>$ & $2.37=$ & $=$ & \\
\hline & targe & $3964=$ & $0:=$ & o. & $2.32 \mathrm{E}-04>$ & $12.0 .>$ & & \\
\hline & Buoyant diap & $0=$ & ol= & $0=$ & $0.00 E+\infty=$ & 이 = & $=$ & \\
\hline & Non-buoyant: & $11885=$ & $72 .<$ & 4) & $1.18 E-\left.03\right|_{2}$ & $2.2=$ & $E$ & \\
\hline$S Y-100$ & all gizes & $4609=$ & $97 \mid<$ & $4 \mid<$ & $5.37 \mathrm{E}-\infty 2 \mathrm{2}$ & 4.037 & & \\
\hline & amall & $1825>$ & 18. $<$ & of $\leq$ & $7.78 \mathrm{E}-03<$ & $0.034<$ & & \\
\hline & modium & $17 t 2>$ & $78 \mid>$ & 4) & $9.99 E-\infty 0>>$ & $3.87<$ & & \\
\hline & large & $1002 .<$ & o|< & $0<$ & $|.18 \mathrm{E}-\infty 03|<$ & $20.61>$ & & \\
\hline & Buoyant diap & $1008=$ & $33<$ & $4 .<$ & B. $.98 \mathrm{E}+00 \mathrm{C}$ & $4.54 ?$ & & \\
\hline & Non-buoyant & $300 t=$ & $50<$ & ol:- & $1.128-02<$ & $3.68]_{2}$ & & \\
\hline s $y-101$ & Lall aizen & $4330=$ & $505:=$ & $23:=$ & $3.95 \mathrm{E} \cdot 03 .=$ & $6.71]_{3}$ & & \\
\hline & smas & $1061=$ & $361=$ & $0 .=$ & 8.68E-04:= & $\begin{array}{ll}0.242= \\
\end{array}$ & & \\
\hline & modium & $2220=$ & $192=$ & $14=$ & $+.38 E+\infty<k$ & $7.511=$ & & \\
\hline & Large. & $10491=$ & $337=$ & $91-$ & $1.2 B E \cdot 0 \mathrm{C}=$ & $70,4 \mid-$ & & \\
\hline & Bucyant diapi & $\left.1336\right|^{2}$ & 35:- & $23 \mid=$ & $1.00 E+01=$ & $7.35=$ & & \\
\hline & Non-buoyami: & $2994=$ & $500=$ & of $=$ & $1.22 \mathrm{E}-09$. & $6.04=$ & & \\
\hline & & & & & & & & \\
\hline intrualve of & Olobatine & No. events & No. deftag. & No. deton. & Median treg. & Median cono. 1 & & \\
\hline AN-107 & at aizee & $|4223|=$ & 22 & a $=$ & $3.50 \mathrm{E}-03$. & $2.38=$ & & \\
\hline & small & $1220=$ & 12. & $0=$ & $1.25 \mathrm{E}-00$ & $0.251=$ & & \\
\hline & medum & $2003 \mid=$ & 80. & a. & $1.57 \mathrm{E}-013$ & $2.68=$ & & \\
\hline & large & $1000 x$ & $0=$ & 이 = & $1,19 E-00$ & $16.4=$ & & \\
\hline & Buoyant displ & $1229=$ & $10=$ & $8 \mid=$ & $6.03 E+01=$ & $2.33=$ & & \\
\hline & Non-buoyant & 2994 . & $76=$ & $0=$ & $1.22 \mathrm{E}-\infty \mathrm{I}_{\mathrm{I}}$ & $2.38=$ & & \\
\hline AW-106 & all ... & $12123>$ & $112>$ & $0=$ & $5.50 E-04 \mid<$ & $2.22=$ & & \\
\hline & small & $4035>$ & $12=$ & $0=$ & $3.51 E-03 \mid<$ & $0.229<$ & & \\
\hline & madium & $40441>$ & $100>$ & 0. $=$ & $9.43 E-04<$ & $2.44 \mid>$ & & \\
\hline & large & $4044>$ & $0 .=$ & 이프. & $1.03 E-04<$ & $13 \mid>$ & & \\
\hline & Exoyant disp & o|: & 0. & 0 & $0,00 E+00=$ & $0:$ & & \\
\hline & Non-buoyant & $12129>$ & $112>$ & 0,0 & $5.50 \mathrm{E}-04<$ & $2.22=$ & & \\
\hline SY-103 & all sizas & $4580<$ & $93<$ & 13) & $5.02 E-09 \leqslant$ & $4.17>$ & & \\
\hline & smah & $1819>$ & $16<$ & $0<$ & $7.60 E-03<$ & $0.774<$ & & \\
\hline & medlum & $1757>$ & $77>$ & $13>$ & 9.83E-03|> & $3.98<$ & & \\
\hline & large & $1004 k$ & $0 \mid<$ & $0<$ & $1.15 k+03 k$ & $20.5>$ & & \\
\hline & Buoyant digp & $1572<$ & $27 \times$ & $13>$ & $3.04 E+\infty 0 \mid>$ & $4.63>$ & & \\
\hline & Non-buoyent & $3008=$ & $86>$ & $0 .=$ & $1,196-036$ & $3.87 / 3$ & & \\
\hline SY-to: & alt sizes & $4279 \leq<$ & $560:=$ & $33>$ & $3.89 \mathrm{E}-03 \times$ & B.6. $<$ & & \\
\hline & small & $10541=$ & $35<$ & $0 \pi$ & B.44E-04< & $0.235 />$ & & \\
\hline & medium & $2179<$ & $183<$ & 20)> & $3.40 E+00>$ & $7.39<$ & & \\
\hline & large & $1048=$ & $345 / 5$ & $13>$ & $1.28 \mathrm{E}-\infty 9=$ & $72.1 \geq$ & & \\
\hline & Buoyani dispef & $1297<$ & $64 \mid \leq$ & $39 j>$ & $1.09 E+0 t]>$ & $\left.7.38\right|_{a}$ & & \\
\hline & Non-buoyant & $2982=$ & $499=$ & $0=$ & $1.23 \mathrm{E}-03 \mathrm{~B}$ & $5.96<$ & & \\
\hline & & & & & & & & \\
\hline Intrusive of & Ex-Tank-inc & \begin{tabular}{|l|} 
No. evonts \\
\end{tabular} & No. doflag. & No. daton. & Medlan freq. & Median cono. & & \\
\hline AN-107 & all sizes & $4223]=$ & $-\quad 92=$ & $\theta=$ & $3.58 \mathrm{E}-03$. & 2.36 & & \\
\hline & Samal & $\left.1220\right|_{x}$ & $12=$ & of $=$ & $1.25 E-09=$ & $0.251=$ & & \\
\hline & medium & $2003=$ & $80=$ & 8. & $7.07 \mathrm{E}-028$ & 2.68 & & \\
\hline & large & $1000=$ & $0=$ & of- & $1.19 \mathrm{E}-100=$ & $16.4 \mid=$ & & \\
\hline & Buoyant diag & $1229 m$ & $16]_{2}$ & $\left.8\right|_{2}$ & $6.08 E+01=$ & $2.33 .=$ & & \\
\hline & Non-buoyant & $2984=$ & $78:=$ & $0 .=$ & $1.22 E-03=$ & $2.38=$ & & \\
\hline AW-108 & atl & $+1811 \mid=$ & $104=$ & $0=$ & $8.36 E-04: 3$ & $2.2=$ & & \\
\hline & amsil & $3931 \mid=$ & $12=$ & 0. & $4.98 \mathrm{E}-03 \mathrm{~J}=$ & $0.239=$ & & \\
\hline & medlum & $3940 \mid=$ & $92-$ & 0 & T.28E- $-0 \mathrm{a} \mid=$ & $2.38 \mid-$ & & \\
\hline & IRrge _.... & $3940]=$ & $0=$ & o. & $1.68 \mathrm{E}-04=$ & $12.8 .=$ & & \\
\hline & Buoyant diapt & 0 & $0:=$ & $0 \mid=$ & $0.00 E+\infty=$ & 0.7 & & \\
\hline & Non-beroyant & $|1811|=$ & $104=$ & $0 .=$ & c.3eE-0a/. & $2.2=$ & & \\
\hline SY-103 & fall slzos & $4638=$ & 95: & $10 .=$ & $3.20 E-03 \mid=$ & 3.96 - & & \\
\hline & $\mathrm{smat}$ & $184 t>$ & $14 \mid<$ & $0<$ & $7.25 \mathrm{E}-\left.03\right|_{<}$ & $0.00705:<$ & & \\
\hline & modium & $1787>$ & $81>$ & $10 \mid>$ & $9.91 \mathrm{E}-03 \mid>$ & $3.83 \mid<$ & & \\
\hline & Large & $1010<$ & $0 .<$ & $0<$ & $1.30 E-\infty 3<$ & 20.4 > & & \\
\hline & Bucryant diap & 1614 a & $34 \mid=$ & $10=$ & $7.71 E+\infty=$ & $4.25=$ & & \\
\hline & Non-buoyant & $30241=$ & 611: & 0. & $1.21 E-\infty)=$ & $3.75=$ & & \\
\hline $5 Y-101$ & all sizas & $43301=$ & 565 - & $23 .=$ & $3.25 E-03 \mid=$ & $6.71=$ & & \\
\hline & small & $1081=$ & 36.-. & of $=$ & 6.70E-0.4 $=$ & $0.242=$ & & \\
\hline & medium & $2220=$ & 192:= & 140. & $2.54 E+\infty 0 \mid-$ & $7.51=$ & & \\
\hline & terge. & 1049:= & $337=$ & 비드. & $1.28 \mathrm{E}-\left.03\right|_{2}$ & $70.4=$ & & \\
\hline & Buoyant dlapi & $1336=$ & $65=$ & $23=$ & $1.05 E+01-$ & $7.35=$ & & \\
\hline & Non-buoyent & $2994=$ & $500=$ & 이- & $1.22 \mathrm{E} \cdot 031-$ & $8.04=$ & & \\
\hline & & 1 & & & 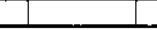 & 1 & & \\
\hline
\end{tabular}




\begin{tabular}{|c|c|c|c|c|c|c|c|c|}
\hline \multicolumn{2}{|c|}{ Ign. Gerl. Sat - In-Tank - } & \multirow{2}{*}{ No. evente } & No. dofiag. & \multirow{2}{*}{\begin{tabular}{|l|} 
No. detonn. \\
\end{tabular}} & \multirow{2}{*}{$\frac{\text { Medien treq. }}{3.58 E-\infty 9}$} & \multirow{2}{*}{$\begin{array}{r}\text { Median nono. } \\
2,36\end{array}$} & \multirow{2}{*}{$=$} & \multirow{2}{*}{ ــ } \\
\hline$A N-107$ & all stzes & & s2i- & & & & & \\
\hline$A W-10 B$ & all sizes: & $11811=$ & 104:m & 이- & Q.38E-04 & $2.2 .=$ & - & \\
\hline $8 \gamma-103$ & at obzes: & 4639:- & $95=$ & $10 \mid=$ & $3.20 E-03$. & 3.96. & + & \\
\hline sY.101 & all sizes & 43300 & $585=$ & $23 .=$ & $3.95 E-03 .=$ & $8.71=$ & \pm & \\
\hline & & & & & & & & \\
\hline \multicolumn{2}{|c|}{ Ion. Ctrl. Set - Ex-Tank - } & No. oventh & \begin{tabular}{|l|l} 
No. deflag. \\
\end{tabular} & No. deston. & Medien treq. & Median cono- & & \\
\hline AN-107 & all sizen. & $4223=$ & 92 $=$ & o. & $3.50 E-03=$ & $2.36=$ & $=$ & \\
\hline AW- 106 & all sizes & $11811=$ & $104=$ & $0=$ & 8.36E-04 $=$ & $2.2 .=$ & $=$ & \\
\hline sY-103 & Ill mixese & 4638]=- & $95-$ & 10-- & $5.20 E-03=$ & $\left.3.28\right|^{*}=$ & 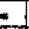 & \\
\hline $8 Y-10 Y$ & all alzea & $43901=$ & 5850 & $23=$ & $3.06 E-08=$ & $9.71=$ & $=$ & \\
\hline & & & & & & & & \\
\hline $\operatorname{lon}, \mathrm{Cor} . \mathrm{s}$ & - in/Ex-Tank: I & No. wents & No. deflag. & \begin{tabular}{|l|l} 
No. daton. & \\
\end{tabular} & Medlen freg. & Madian conc. & & \\
\hline AN-107 & all oizes & $4223=$ & 92. & 8. & $3.505-0 \mathrm{~g}$ & $2.36=$ & 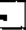 & \\
\hline$A W-108$ & all sizes & $11811=$ & 104:- & o..- & $0.36 E-04$ & $2.2=$ & 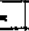 & \\
\hline SY-100 & al oize: & $4838=$ & $85=$ & $10,=$ & $\$ .206 .08=$ & $3.95=$ & 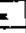 & \\
\hline SY-101 & all nizas & $4330=$ & 5605 & 23... & $3,85 \mathrm{E}-03=$ & $6.71=$ & 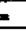 & \\
\hline & & & & & & & & \\
\hline Density is: & Leq to 5. Sol & No. events & \begin{tabular}{|l|} 
No. deflag. \\
\end{tabular} & No. deton. & Medlun treq. & Median sone. & & \\
\hline $\mathrm{AN}-107$ & Fill alzat & $4354>$ & $|1109|>$ & 53 & $|3.92 E-\infty|>$ & $10.81>$ & $>$ & \\
\hline & Buoyant diap & $1356 />$ & $155>$ & $52>$ & $7.33 \mathrm{E}+00<<$ & 10. & $\geq$ & \\
\hline & Non-buoyant & $2998=$ & $954>$ & $? \geq$ & $1.21 E-03=$ & $21.5>$ & $\geq$ & \\
\hline$A W=10 B$ & all sizes & $3950<$ & $1048>$ & $45>$ & $2.92 E-031>$ & $28>$ & 2 & \\
\hline & Buoyant diapl & $1040>$ & $2533 \times$ & $45 \mid>$ & $1.51 E-01$ & 32.312 & 2 & \\
\hline & Non-buoyant & $2910<$ & 70813 & $0 .=$ & $1.18 \mathrm{E}-\infty)>$ & $29.81>$ & $>$ & \\
\hline sY-103 & all sizas & $4644<$ & $1131 / 3$ & 512 & $4.46 E-09 \mid<$ & $27.7>$ & 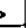 & \\
\hline & Buoyant dlapt & $1481<$ & $191 \mid>$ & $51 \mathrm{~s}$ & $1.20 \mathrm{E}+00 \mathrm{~K}$ & 27.37 & 2 & \\
\hline & Non-buoyani! & $3083>$ & 940 > & $0=$ & $1.24 E-09>$ & $28>$ & $>$ & \\
\hline $5 Y \cdot 101$ & at elzes & $4233 \mid<$ & 1174 & $457>$ & $3.44 E-09<$ & $40.1 \mathrm{P}$ & 2 & \\
\hline & Buoyant diop & $1245 \leqslant$ & $275 \mid>$ & $8 \theta>$ & $2.24 \mathrm{E}+00 \mathrm{C}$ & $42.7 / 2$ & \pm & \\
\hline & Non-buroyant & 2908) $=$ & 8998 & $350>$ & $1.21 \mathrm{E}-03=$ & $38>$ & $>$ & \\
\hline & & & & & & & & \\
\hline Density is & Uq to l.Sol & \begin{tabular}{|l|l} 
No. events & \\
\end{tabular} & \begin{tabular}{|l|l|} 
No. delleg. \\
\end{tabular} & \begin{tabular}{|l|l|} 
No. delon. \\
\end{tabular} & Medlan frea. & Median cone. & & \\
\hline$\overline{A N}-107$ & all sizes & $4223=$ & 92 $=$ & 8:- & $3.54 E-03=$ & $2.36=$ & $=$ & \\
\hline & Buoyant dfapt & $1228=$ & $181=$ & 8)= & 6.00E+01 = & $2.33=$ & \pm & \\
\hline & Non-bucyant & $2894[=$ & $76=$. & 0: $=$ & $1,22 E-\infty)=$ & $2.38=$ & $=$ & \\
\hline$A W-106$ & 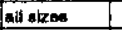 & $|11811|=$ & $104 \mid=$ & 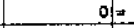 & $9.38 E-0 \mathrm{~s}=$ & 2.21 & 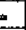 & \\
\hline & Buoyant diap & ole & 이 & o) $=$ & $0.00 \mathrm{E}+\infty$. & $0 \mid=$ & \pm & \\
\hline & Non-buoyant & $11811=$ & 704. & $0:=$ & $8.38 E-0<$ & $\left.2.2\right|_{1}$ & \pm & \\
\hline $5 Y-103$ & \begin{tabular}{|l|l|} 
ata sizes \\
\end{tabular} & $4638=$ & $95 / \pi$ & $10=$ & $5.20 E-03$ & $3.20 / \mathrm{m}$ & 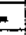 & \\
\hline & Buoyent dlepel & $1814=$ & $34=$ & $101=$ & $7.71 \mathrm{E}+\infty$ & $4.25=$ & 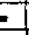 & \\
\hline & Non-buoyan! & $3024 \times$ & $61=$ & $0:=$ & 1.21E-03 & $3.75=$ & $\pi$ & \\
\hline$S Y-101$ & nil alzen & $4233,<$ & $1+74>$ & $457 ?$ & $3.44 E-03$ & $40.1 />$ & $\geq$ & \\
\hline & Buoyert diso: & $1338=$ & Gs a & $23=$ & $1.05 E+01$ & $7.35:=$ & 3 & \\
\hline & Non-tuoyant & $2984=$ & $500=$ & $0 .=$ & $1.222 E-03=$ & $5.04=$ & $\pi$ & \\
\hline & & & & & & & & \\
\hline Gas gene & on-S.Sol-inc & No. wents & No. deflug. & No, daton. & Madian freq. & Median conc. & & \\
\hline AN-107 & at skizas & $4223=$ & $92 .=$ & $8=$ & $3.58 E-\infty 8$ & $2.30=$ & $=$ & \\
\hline & small & $1220=$ & $12=$ & $0=$ & $1.25 E-08$ & $0.251=$ & $=$ & \\
\hline & medtum & $20003=$ & $60 \mathrm{~m}$ & $8 \cdot x$ & $7.87 E-\infty 2=$ & $2.08=\pi$ & $x$ & \\
\hline & large & $1000=$ & $0=$ & $0]=$ & $1.19 E-09$ & $16.4:=$ & 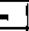 & \\
\hline & Buoyant dispo & $1229=$ & 16 - & 8. $=$ & $6.11 \mathrm{E}+0.2$ & $2.33=$ & $=$ & \\
\hline & Non-bwoyant & $2994=$ & $78=$ & $0 \mid=$ & $1.22 \mathrm{E}-03$ & $2.38=$ & \pm & \\
\hline AW.108 & an & $12016>$ & $08<$ & ofl. & $2.82 E-\infty, 3$ & $2.18 \times$ & 2 & \\
\hline & smail & $4000>$ & 18 $>$ & $0=$ & $1.93 \mathrm{E} .02$ & $0.23 \times 1<$ & $\leq$ & \\
\hline & mexium & 4000 ; & $72<$ & 이 = & 5.68EE-00:3 & $2.31<$ & $<$ & \\
\hline & lenrgo & 40003 & 0. & of = & $5.06 E-04$ ? & $12.6=$ & 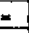 & \\
\hline & Buoyent dlap: & 0 & 이 & of. & $0.00 E+00$ & & 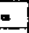 & \\
\hline & Nonbuoyent & $12016>$ & 881< & $0=$ & $2,82 E-03$, & $2.16<$ & $<$ & \\
\hline $5 Y=103$ & all sizes & 4602 & 91k & $11 />$ & 5.25E-D3. & $3.80 \mid \mathrm{m}$ & $m$ & \\
\hline & emall & $18191>$ & $18<$ & $0<$ & $7.43 E-095$ & $0.785 \mathrm{~K}$ & $\leq$ & \\
\hline & medium & 1783, & $72>$ & $11>$ & B..86E-02,3 & 3.896 & $\leq$ & \\
\hline & large & $10001<$ & ole & $0 . k$ & $1.10 E-\infty 9$ & 20,713 & $\geq$ & \\
\hline & Busoyant diapl & $1600 \mid=$ & $31 /<$ & $11 />$ & $6.04 E+01$ & $4,38>$ & $>$ & \\
\hline & Non-buoyant & $2994=$ & $\Delta 0 k$ & 0 of- & $1.21 E-\infty$ & $3.78=$ & 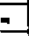 & \\
\hline SY.101 & all sires & $4331 \mid=$ & 5.55. & $23=$ & $3.95 \mathrm{E}-0.03$ & 6.7.- & 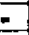 & \\
\hline & small & $1001=$ & $36=$ & o:- & B.60E-04 & $0.242=$ & $E$ & \\
\hline & medium & $22211=$ & $192[=$ & $14=$ & $2.78 E+\infty 00$ & $7.51=$ & $=$ & \\
\hline & large & $1049=$ & $337]_{-1}$ & $9 .=$ & $1.28 E-\infty$ & $70,41 \mathrm{~m}$ & $m$ & \\
\hline & Buoyant diapl & $1337=$ & $66)$ & $23:=$ & $1.00 E+02$ & $7.35=$ & 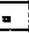 & \\
\hline & Non-buoyant & $2994 \mid=$ & 5000 & $0=$ & $1.22 E-03$ & $0.04=$ & - & \\
\hline & & & & & & & & \\
\hline
\end{tabular}




\begin{tabular}{|c|c|c|c|c|c|c|c|c|}
\hline \multicolumn{2}{|c|}{ Gas generation + .Sol-inc } & \multirow{2}{*}{\begin{tabular}{|r|} 
No. evente \\
4223 \\
\end{tabular}} & \multirow{2}{*}{\begin{tabular}{|r|} 
No. doflag. \\
92 \\
\end{tabular}} & \multirow{2}{*}{ 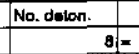 } & \multirow{2}{*}{\begin{tabular}{|r|} 
Median freq. \\
$3.58 E-09$
\end{tabular}} & \multirow{2}{*}{\multicolumn{2}{|c|}{\begin{tabular}{|}
$\mid$ Median cone. \\
$2.38 \mid$
\end{tabular}}} & \multirow{2}{*}{ 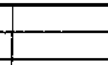 } \\
\hline AN-107 & all stzes & & & & & & & \\
\hline & smat & $1220=$ & $12=$ & of = & $1.25 E-03=$ & $0.251=$ & & \\
\hline & medium & $2003=$ & so $=$ & B) $=$ & $7.87 E-02=$ & $2.66=$ & & \\
\hline & lewge & $1000=$ & ol- & $0=$ & $1.19 \mathrm{E}-00=$ & $16.4=$ & & \\
\hline & Buoyant diapl & $1228=$ & $16 \mathrm{I}_{\mathbf{E}}$ & B: $:=$ & $8.00 E+01=$ & $2.33=$ & & \\
\hline & Non-buoyant & $2904=$ & $76=$ & 요 = & $1.22 \mathrm{E}-03=$ & $2.30 \mid=$ & & \\
\hline \multirow[t]{6}{*}{$A W-10 B$} & all & $11814=$ & $104=$ & 이 $x$ & $0.30 E-04:=$ & $2.21=$ & & \\
\hline & smoli & $3831=$ & $12=$ & a) & $4.98 \mathrm{E}-096$ & $0.238 .=$ & & \\
\hline & medium & $3940=$ & $92=$ & $0=$ & $1.20 \mathrm{E}-\infty \mid-$ & 2.38 & & \\
\hline & large & $3840-$ & 0) $=$ & $0=$ & $1.68 E-04=$ & $12.6=$ & & \\
\hline & Buoyant diapt & 이 & 0. $=$ & of:- & $0.00 \varepsilon+00=$ & $0=$ & & \\
\hline & Nen-buoyant. & \begin{tabular}{l|l|}
11811 & $n$ \\
\end{tabular} & $104=$ & $0 .=$ & B.38E-04:= & \begin{tabular}{l|l}
2.2 & $=$ \\
\end{tabular} & & \\
\hline \multirow[t]{6}{*}{$\$ Y .103$} & ail sizes & $4802=$ & $91 k$ & 11) $>$ & $5.25 E-03$. & $3.98=$ & & \\
\hline & amaih & $1810>$ & $18<$ & $0<$ & $7.43 \mathrm{E}-\infty 91 \mathrm{~K}$ & $0.755<$ & & \\
\hline & medium & $1793>$ & $72>$ & $11>$ & $9.86 \mathrm{E}-03 />$ & $3.89<<$ & & \\
\hline & large & $1000<<$ & $0:<$ & $01<$ & $1.19 \mathrm{E} \cdot 03 .<$ & 20.75 & & \\
\hline & Buopant diap & $180 \mathrm{a}$ & $31 \mid<$ & $11 / 2$ & $8.26 E+001>$ & $4.38>$ & & \\
\hline & Non-buoyant & 2994 [- & $80 \mid<$ & $0=$ & $1.21 \mathrm{E}-\infty$ & $3.78=$ & & \\
\hline SY-101 & ail sizes & $4330=$ & $565:$. & $23=$ & $3.95 E-03 \mid=$ & $6.71=$ & & \\
\hline & amait & $1081 \pi$ & $38=$ & a. $=$ & $8.86 \mathrm{E}-04=$ & $0.242=$ & & \\
\hline & medium & $2220=$ & $192=$ & $14=$ & $2.54 E+00=$ & $7.51=$ & & \\
\hline & large & $1049 . \mathrm{I}$ & $337]=$ & $9=$ & $1.28 E-03=$ & $70.4 \times$ & & \\
\hline & Buoyam digp & $1336=$ & $85=$ & $23=$ & $1.05 E+011=$ & $7.351=$ & & \\
\hline & Non-buoyant & $2994=$ & $500 \mid=$ & of- & $1.22 E-03=$ & 6.04 & & \\
\hline & & & & & & & & \\
\hline Tank fail pres & as red. & No. events & \begin{tabular}{|l|} 
No, defing. \\
\end{tabular} & No, deton. & Median freq. & Medlan cons. & & \\
\hline AN- 107 & all aizea & $4223=$ & 92: & $\theta=$ & 3.5BE-00 $\times$ & $2.38=$ & & \\
\hline AW-106 & :all sizba & $11811=$ & $104=$ & $a=$ & $8.36 \mathrm{E}-04=$ & 2.2. & & \\
\hline SY-103 & Ell sizes & $4630=$ & $95=$ & $10=$ & $5.20 \mathrm{E}-03=$ & $3.981 \times$ & & \\
\hline$S Y \cdot 10 t$ & all size: & $4330 \mid=$ & $525:=$ & $23 .-$ & $3.95 E-03=$ & $6.711=$ & & \\
\hline & & & & & & & & \\
\hline WIE OPs ino & & No. events & No. deflag. I & No. deton. & Median treq. & Median cono. & & \\
\hline$A N \cdot 107$ & all sizes & $4252=$ & $117 />$ & $12>$ & $3.80 E-091>$ & $2.41 \times$ & & \\
\hline & WIEquip \{all : & 29 & $25>$ & 45 & $1.00 E+\infty 0$ & $134>$ & & \\
\hline$A W-100$ & all sizes _. & 11814= & $105=$ & $2>$ & 8.38E-04 $=$ & $2.2=$ & & \\
\hline & WIEquip (all s & 0.1- & $0 \mid=$ & $0=$ & $0,00 E+00 \mid=$ & ofe & & \\
\hline SY- $\neq 03$ & all sizes & $4847=$ & $95=$ & $19>$ & $5.23 E-09=$ & $3.99-$ & & \\
\hline & WIEquip (all \& & $9>$ & $0=$ & $9>$ & $1,005+00$ & $124>$ & & \\
\hline SY-101 & all sizes & $4349=$ & $574>$ & $33>$ & $4,00 E-03>$ & $6.79>$ & & \\
\hline & WIEquip (alls & 193 & 9) & $10 \geq$ & $1.00 E+00>$ & $127>$ & & \\
\hline & & & & & & & & \\
\hline Whe Ops ine- & ign.ctr & No. events & No. deflad. & \begin{tabular}{|l|l} 
No. detor. \\
\end{tabular} & Median trea. & Meden cono. & & \\
\hline AN=107 & at sizes & $4771>$ & 543 & $105 i>$ & $6.25 E-03\}$ & 3.39 & & \\
\hline & WiEquip (aill s & $548>$ & $4511>$ & 973 & $1.00 \mathrm{E}+\infty 0 . \mathrm{z}$ & $218>$ & & \\
\hline AW-10B & alt sizos & $12860 \mathrm{~s}$ & 5001 & $351>$ & $1.15 E-03\}>$ & $2.82>$ & & \\
\hline & WIEquip (페 s & $0=$ & $0=$ & $0=$ & $0.00 E+00=$ & 이 & & \\
\hline $5 Y-103$ & all sixba & 5360 ? & $472>$ & 3832 & 8.82E-0.09> & 5.95 & & \\
\hline & WIEquip (all $\&$ & $730>$ & $3 n>$ & 353 ? & $1.00 E+00=$ & $242>$ & & \\
\hline SY-101 & All size & 4897> & $964>$ & $291>$ & $7.55 \mathrm{E}-03>$ & $10.3>$ & & \\
\hline & WIEquip (alls & $687>$ & $398>$ & $260 \%$ & $1.00 E+00=$ & $258>$ & & \\
\hline & & & & & & & & \\
\hline WIE CPs inc- & equip dia & No. events & \begin{tabular}{|l|l} 
No. deflag. \\
\end{tabular} & No. dabon. & Median treq. & Median conc. & & \\
\hline AN 107 & ail aizes & $4252=$ & $117=$ & $12=$ & 3.69E-03:- & \begin{tabular}{ll|l}
2.41 & $\mathrm{n}$ \\
\end{tabular} & & \\
\hline & WIEquip (ails & 29. & $25=$ & 4. & $1,00 E+\infty)=$ & $134:=$ & & \\
\hline AW-106 & ail stzes & $11814=$ & $105:=$ & $2=$ & $8.30 E-04=$ & $2.2 .=$ & & \\
\hline & WIEquip (all s) & of = & 이 & 의= & $0,00 \mathrm{E}+\infty)=$ & $0 ; 0$ & & \\
\hline$S Y \cdot 103$ & all sizes & $\left.4647\right|_{=}$ & 95:1: & 191. & $5.23 \mathrm{E}-00=$ & $3.99=$ & & \\
\hline & WIEquip (all s & $9=$ & $0=$ & $8=$ & $1,00 E+00 \mid=$ & $124=$ & & \\
\hline $5 \gamma-101$ & all sizas & $4348=$ & $574=$ & $33=$ & $4.00 \mathrm{E}-00=$ & $6.79=$ & & \\
\hline & WIEquip (all है & $181=$ & $9=$ & $10=$ & $1.00 E+00=$ & $127 \mid=$ & & \\
\hline & & & & 1 & & & & \\
\hline WIE Opa ine- & equip lon & No. events & No. daftag. & No. deton. & Median freq. & Median conc. & & \\
\hline AN-107 & all sizes & $4252=$ & $117 \mid \pi$ & 12:- & $3.69 E-03]=$ & $2.411=$ & & \\
\hline & WiEqup (all s & $29=$ & $25=$ & $4 \times$ & $1.00 \mathrm{E}+00 \mathrm{O}=$ & $134=$ & & \\
\hline AW.106 & ah sizes & $17814: 0$ & $105=$ & $2=$ & $0.38 E-04=$ & $2.21=$ & & \\
\hline & WIEquip (al) \& & $0=$ & 0. & $0:=$ & $0.00 E+00=$ & $0 \mid=$ & & \\
\hline$s Y-103$ & \begin{tabular}{|l|l|} 
all sizes \\
\end{tabular} & $4847=$ & $95:=$ & $19=$ & $5.23 E-00=$ & 3.29 & & \\
\hline & WIEquip (ail s & 9. & $a=$ & 910 & $1.00 \mathrm{E}+\infty \mathrm{x}$ & $124=$ & & \\
\hline $5 Y-101$ & all gizes & $434 \theta$ & $374 \mid x$ & 331. & $4.00 E-03=$ & $8.79=$ & & \\
\hline & WIEquio (all : & 190 & $9 \mid=$ & $10=$ & $1.00 \mathrm{E}+\infty 0 \mathrm{i}=$ & $127=$ & & \\
\hline
\end{tabular}




\begin{tabular}{|c|c|c|c|c|c|c|c|c|c|c|c|c|c|c|}
\hline & & & & & & & & & & & & & & \\
\hline & & & & & & & & & & & & & & \\
\hline & & & & & & & & & & & & & & \\
\hline \multicolumn{2}{|c|}{ Benohmerk - On-site Riad } & & & & & & & & & & & & & \\
\hline Tank & & & & & & & & & Benohmark - C & On-aite Tox & & & & \\
\hline$A N-107$ & allTS & 100 & & $1.46 E-05$ & $\frac{\text { Modlan cons. }}{2.75 \mathrm{E} \cdot 10}$ & & \begin{tabular}{|l|} 
Ex, Rlak \\
$5.03 E-\infty$
\end{tabular} & & \begin{tabular}{|l} 
Tank \\
AN-107
\end{tabular} & all TDS & \begin{tabular}{|l|l|} 
No. events & \\
$100 / 8$
\end{tabular} & \begin{tabular}{|c|} 
Medien freq. \\
$.46 \mathrm{EE}-05$
\end{tabular} & $5.03 E-06$ & \\
\hline & Onatto Red: & 621 & & 1.00E-0s B & $2.38 \mathrm{E}-11$ & & $4.00 E-00 \mathrm{~B}$ & & & Oneite Tox: & 828 & $1.00 E-05$ B & $5.20 \mathrm{E}-07$ & $7 \mathrm{~B}$ \\
\hline & Onolite Aad: & $3: \mathrm{B}$ & & 2.90E-01 B & 7.,67E-06 & & $1.73 E-05.6$ & & & Onsite Tax; & 318 & $2.905-01 / B$ & $3.71 E+00$ & 0 \\
\hline & Oneide Aad: & 15. E & & 3.03E-01 B & $6.39 E-05$ & & 3.97E- $-\infty: B$ & & & Onaile Tox: & $18: 8$ & $3,03 E-01 B$ & $5.24 \mathrm{E}+01$ & 1.9 \\
\hline$A W-106$ & at Tos & $104: 1$ & & $3.07 E-06$ & 8.82E-10 & B & $5.44 E-\infty 9$ & & AW-108 & all Tos & $104 / \mathrm{B}$ & $3.07 \mathrm{E}-08 \mathrm{~B}$ & 9,87E- $\infty 6$ & \\
\hline & Onaite Rad: & Q41: & & $2.39 \mathrm{E}+06$ & $4.60 E-12$ & 8 & Q.17E-11 8 & & & Onsite Tox: & $84 \mathrm{~B}$ & $2.30 \mathrm{E}-08 \mathrm{~B}$ & $2.38 E-\infty 8$ & \\
\hline & Onaite Rent: & $10 \mid \mathrm{B}$ & & $2.026-051 \mathrm{~B}$ & $7.006-04$ & $B$ & \begin{tabular}{ll|l}
$.50 E-008$ & $B$ \\
\end{tabular} & & & Onaite Tox: & $10 B$ & $1.82 E-05,8$ & 1.37E+011 & $1 \mathrm{~s}$ \\
\hline & Onalte Rad: & 24 & & $1.04 E-068$ & $5.30 E-06$ & & $3.03 E-07 / 3$ & & & Crolte Tox: & $24 B$ & $1.64 E-00 ; B$ & 1.09E-01 & \\
\hline sY-103 & all TOS & 105 & & 2.45E-05:B & $2.18 E-04$ & 8. & $3.39 \mathrm{E}-05 / \mathrm{B}$ & & sY.103 & at Tos & $105 \mathrm{~B}$ & $2.45 E-05 B$ & 4.90E-01:E & \\
\hline & Onaite Pad: & $15 \mid \mathrm{E}$ & & $1.53 \mathrm{E}-031 \mathrm{~B}$ & $4.19 E \div \div 0$ & & $4.385-0.06$ & & & Onaite Tox: & 15. $B$ & $1.53 \mathrm{E}-03 \mathrm{~B}$ & $4.37 \mathrm{E}-08$ & \\
\hline & Ontaile Rad: & 39 & & $7,32 E-06$ & $2.87 E-94$ & B & $4.25 E-0418$ & & & Onsite Tox: & $30 \mathrm{~B}$ & $7.32 E-06]$ & 3.94E-01 & \\
\hline & Onalle Rad: & $51 \mathrm{E}$ & & $4,41 \mathrm{E}-04 \mathrm{E}$ & $8.28 \mathrm{E}-0.4$ & B & 1.3BE.03:B & & & Onsite Tox: & $511 \mathrm{~B}$ & 4.4TE-04] & 7.22E+0O & \\
\hline sY-101 & aH TOS & 589.8 & & $1.24 \mathrm{E}-04 \mathrm{~g}$ & $1.16 \mathrm{E}-05$ & B & 4.74E-09: & & $8 Y-10 t$ & Alf TDS & $588 / B$ & $1.24 E-0410$ & $3.08 \mathrm{E}-\infty 2$ & \\
\hline & Ongile And: & 275 & & $4.70 E-056$ & $1.59 \mathrm{E} \cdot 06$ & B & 9.57E-071日 & & & Oneite Tox & 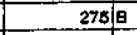 & $4.70 E-0510$ & 4.41E- -05 & \\
\hline & Onsito Rad: & 184 & & $1.59 \mathrm{E}-04 \mathrm{~B}$ & 2.43E-04 & $B$ & $7.69 \mathrm{E}-\infty) \mathrm{B}$ & & & Onolite Tox: & 1846 & $1.58 \mathrm{E}-04 \mathrm{~B}$ & 4.39E-01 & \\
\hline & Onaite Rad: & 129 & & $3.40 E-04$ B & 9.02E.04 & B & 3.36E.02 日 & & & Onsite Tox: & 129|日 & $3.4 C E-04 / B$ & $5.58 E+\infty$ & olg \\
\hline WIE AN $\mathrm{r}$ & & $0 \mid 1$ & & $0.00 E+00 B$ & $0.00 E+00$ & $B$ & $0.00 \mathrm{E}+00 \mathrm{E}$ & & WIE All Tanka & & 0,0 & $0.00 \mathrm{E}+00 \mathrm{~B}$ & $0.00 \mathrm{E}+\infty \mathrm{E}$ & 8 \\
\hline & & & & & & & & & & & & & & \\
\hline & & & & & & & & & & & & & & \\
\hline inerilng-5 & Rad & No. event & & Median treq. & Medien cons. & & Ex, Rak & & inerting-5\% - 7 & Tox & No. ovents & Modian freq. & Median cons. & \\
\hline$A N-107$ & all Tos & $100-$ & & $1,46 E-0.05=$ & 2.03E.11 & 5 & 5.91E- -0 i $>$ & & AN-107 & all TDS & $100=$ & $1,46 \mathrm{E}-05=$ & $5.82 E-07$ & \\
\hline AW- 106 & All TOS & 325 & & $1.02 E-06>$ & $0.00 E-05$ & & 6.27E-00 > & & AW-10B & AITOS & $32<$ & 1.02E-05> & 3.a1E-01: & \\
\hline$S Y-100$ & all TDS & 88 & & 1.33E.05 $<$ & $6.54 E .05$ & & $1.35 E-05<<$ & & $8 Y-103$ & all TDS & $86<$ & $1.33 \mathrm{E}-05<$ & Q. $\mathrm{BaE}-02$ & \\
\hline SY.101 & all TDS & 530 < & & $1.00 E-04 \leq$ & 7.47E-06 & & $4.80 E-\infty>$ & & SY-101 & all TOS & $508=$ & 1.24E-04.- & $3.00 \mathrm{E}-\infty 2$ & 2:- \\
\hline & & & & & & & & & & & & & & \\
\hline Inerting-1 & - Rad & jo. ovent & & Medlen treq. & Median conc. & & Ex Pink & & Inerting-10\% - & Tox & No. wents & Modian freq. & Median cone. & \\
\hline AN-107 & All TDS & $100=$ & & $1.46 \mathrm{E}-05$ & 2.03E-11 & $\leq$ & 3.91E-05 > & & AN-107 & all TOS & $100=$ & $1.48 \mathrm{E}-0 \mathrm{0}=$ & 5. $52 E-07$ & tk \\
\hline$A W-106$ & all TDS & 32 & & $1.02 E-05>$ & $8.806-05$ & & 6.27E-09> & & $A W=106$ & all TDS & $32<$ & $1.02 E-05>$ & $3.61 \mathrm{E}-02$ & is \\
\hline$s \gamma \cdot 109$ & All TOS & 105:- & & 2.45E-05= & 1.21E-04 & & $4.506-0.5 \mathrm{~s}$ & & $8 \gamma-103$ & all TDS & $105=$ & $2,45 E-05=$ & $3.05 E-0.1$ & $1<$ \\
\hline $3 Y-101$ & All TDS & $591=$ & & 1.25E.04 $=$ & $8.92 E-06$ & & $4.75 E-\infty 3: 2$ & & sY-101 & all TDS & $508=$ & $1.24 E-04=$ & $3.08 \mathrm{E}-\mathrm{A2}$ & $=$ \\
\hline & & & & & & & & & & & & & & \\
\hline Waste vo & d. - Rsd & No. events & & Medien fres. & Mectlan cons. & & Ex. Risk & & Waste vol, red & d. - Tox & \begin{tabular}{|l|l} 
No. events. \\
\end{tabular} & Median freq. & Median coma. & \\
\hline$A N=107$ & ail TOS & 89 & & $1.34 E-0 S<$ & \begin{tabular}{|l|}
$5.07 E \cdot 11$ \\
\end{tabular} & & $\mid 5.58 \mathrm{E}-0.06<$ & & AN-107 I & all TDS & 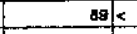 & $1.34 \mathrm{E}-08 \mathrm{~s}$ & 3.62E-07 & $7 \leq$ \\
\hline & Onoile Rad: & 75 & & $9.80 \mathrm{E} .06<\mathrm{C}$ & 5.16E-13. & & $1.73 \mathrm{E}-00<$ & & & Onsite Tox: & $75 k$ & 9.80E-08 K & $3.32 E \cdot 09$ & e \\
\hline & Onsite And: & 2 & & $6.44 E+00)>$ & $1.18 E \cdot 04$ & & $3.03 E-00>$ & & & Onsile Tox: & $2 \mid<$ & $6.44 \mathrm{E}+\infty)>$ & $1.51 E+02$ & \\
\hline & Onaite Rad: & $12<$ & & $4.72 E .01>$ & 8.93E-0. & 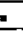 & $4.01 \mathrm{E}-03 \mathrm{~s}$ & & & Onsite Tox: & $12 \mid<$. & $4.72 E-01>$ & $4.84 \mathrm{E}+01$ & $1 k$ \\
\hline AW-106 & al TDS & 725 & & $3.42 E-06$ & $4.37 E-00$ & & $9.81 E-10 \leq 5$ & & $A W-10 B$ & all TDS & $72 \mid<$ & $3.42 E-08$ & 4.60E-C4! & \\
\hline & Onalte Red: & 44 & & 7.23E-07 $<$ & $5.00 E-24$ & & $3.7 \mathrm{EE}-08)>$ & & & Onstite Tax: & $44<$ & $7,93 \mathrm{E}-07 \mathrm{~K}$ & 8.21E-20| & \\
\hline & Onalle Rad: & $A B=$ & & $1.11 \mathrm{E}-05 \mathrm{~S}$ & 2.29E.06 & & 1.15E-07 $<$ & & & Onaile Tox & $16 \mid=$ & $1.11 \mathrm{E}-06<$ & 1.37E-01 & \\
\hline & Onatie Rad: & 12. & & $8.09 E-06$ > & 2.08E-06 & $\leq$ & 1.57 E $-07<$ & & & Onsils Tox: & $12<$ & $8.09 \mathrm{E}-08>$ & 2,04E-01 & \\
\hline SY-100 & all TOS & 82 & & $2.08 E-055$ & 8.43E.05 & $\leq$ & $9.83 E-04>$ & & $\$ Y \cdot 103$ & all TOS & $52<$ & $2.08 E-05<$ & 2.27E.01 & \\
\hline & Ongilt Ped: & 20 & & Q.13E-06 5 & $1.88 E-\infty$ & $\geq$ & $2.60 E-10<$ & & & Onaits Tox: & $20>$ & 8.13E-08< & t.10E-0s & \\
\hline & Onatip Rand: & 28 & & $1.94 E-05$ & 9.8BE.05 & & 4. $34 \mathrm{E}-04>$ & & & Onsite Tox & $20<$ & $1.84 E-05$ & 2.BBE-011 & \\
\hline & Onsito Rad: & 34 & & $5.26 \mathrm{E} \cdot 05$ & $5,78 E-04$ & 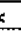 & 8.82E-02 $>$ & 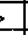 & & Onsile Tox: & $34 \mid \leq$ & $5.25 \mathrm{E}-0.5 \times$ & $4.61 E+00$ & \\
\hline sY-101 & all TDS & $280<$ & & $4.47 \mathrm{E}-05$ & $5.21 E-06$ & 5 & $5.39 \mathrm{E}-\infty 9>$ & 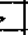 & $S Y \cdot 101$ & all TDS & $280<$ & $4.47 \mathrm{E}-05<$ & $1,16 \mathrm{E}-04$ & $4<$ \\
\hline & Onsite Rad: & 203 & & $2.47 \mathrm{E}-05$ & $7.85 E-09$ & & $5.81 E-07 \mid<$ & $\leq$ & & Oneith Tox: & $203 /<$ & $2.47 \mathrm{E}-05<$ & $1.75 \mathrm{E}-05$ & \\
\hline & Onsite Aad: & 27 & & 4.78E-04) & $5.61 E-04$ & & $5.48 E .00<$ & $\leqslant$ & & Onaile Tox: & $27<$ & 4.78E-04> & 8.84E+00 & \\
\hline & Onsitte Rad: & $301<$ & & 5.14E-02, & $2.00 E-04$ & & $3.38 E-02=$ & 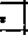 & & Onaite Tox: & $30<$ & $5.14 E-22>$ & $3.29 E+01$ & $1>$ \\
\hline & & & & & & & & & & & & & & \\
\hline Vent. rate & d. Fand & No. evente & & Median treq. & Median cons. & & Ex. Alikk & & Vent rato-rod & - Tox & \begin{tabular}{|l|l} 
No. wants \\
\end{tabular} & Modian freq. & Madian const. & \\
\hline$A N-107$ & all TDS & $152>$ & & $2.31 \mathrm{E}-04$ & $9.10 \mathrm{E}-\infty 9$ & 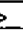 & $9.06 \mathrm{E}-05>>$ & $\geq$ & $A N-107$ & all TOS & $152 \mid \geq$ & $2.31 E-04 / 3$ & $2.22 E-04$ & 4 \\
\hline AW-10B & all TOS & 104: & & $3.07 E \cdot 08$ & $8.92 E-10$ & $a$ & 5.44E-09 = & $\approx$ & AW-10B & all Tos & $104=$ & 3.07E-06|a & $0.67 \varepsilon-08$ & $=$ \\
\hline SY- 103 & ail TDS & $143>$ & & $1,58 \mathrm{E} \cdot \omega)$ & $2.11 \mathrm{E}-04$ & & 8.58E-04? & $>$ & $5 Y-103$ & all TDS & 1433 & $1.59 \mathrm{E} \cdot 03 \mid \mathrm{S}$ & $1.20 E+\infty$ & $0)$ \\
\hline$S Y-101$ & all TOS & $980>$ & & $3.44 E-04>$ & 6.29E-0.5 & 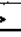 & 6.91E- $0 R>$ & $>$ & $s Y-101$ & all ros & $800 />$ & $3.44 E-04>$ & 2.34E-01 & $1 / 3$ \\
\hline & & & & & & & & & & & & & & \\
\hline Vent rate & Rad & No. events & & Median trea. & Mectian cons. & & Ex. Alik & & Vent rato-inc. & Tox & Na, wents & Median frea. & Medisen cons. & \\
\hline AN-107 & all TDS & at & & $1.24 \mathrm{E}-05$ & $2.00 \mathrm{E} \cdot 11$ & $\leq$ & $4,91 \mathrm{E}-0 \mathrm{~s}<\mathrm{K}$ & & $A N-107$ & EA TOS & $\quad 89<$ & $1.24 \mathrm{E}-05$ & 3.20E-07 & \\
\hline AW-10B & all TDS & $104:$ & & $3.07 \mathrm{E}-06$ & $8.92 \mathrm{E}-10$ & 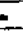 & $5.42 E-\infty 9=$ & & AW-108 & all TOS & $104=$ & 3.07E-06- & 9.67E-09: & $\theta=$ \\
\hline SY-103 & ail TDS & 75 & $\leq$ & $1.07 \mathrm{E}-052$ & 2.00E-04 & $\leq$ & $1.05 E-\infty 6<$ & $\leq$ & $s Y-100$ & antos & $75<$ & $1.07 \mathrm{E}-05 \mathrm{~K}$ & $3.90 \mathrm{E}-01$ & $1<<$ \\
\hline SY-101 & all TDS & 370 & & $6,17 \mathrm{E}-05<$ & 4.04E- -07 & $\leq$ & $4.42 E-04<$ & $\leq$ & SY-101 & All TDS & $370<$ & 6.17E-05:< & 1.17E. 03 & $3<$ \\
\hline & & & & & & & & & & & & & & \\
\hline Int aps-A & o- Plad & No. wents & & Median frea. & Median cons. & & Ex. Alak & & Int ops-Al inc & c. Tox & No. events & $\begin{array}{l}\text { Median freq. } \\
\end{array}$ & Medien oono. & \\
\hline$A N-107$ & all TOS & 100 & 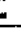 & 1.48E-OS & $2.73 E-10$ & & $5,89 \mathrm{E}-05>$ & & AN-107 & all TDS & $100=$ & $\mid-1.46 \mathrm{E}-05=$ & $5.03 E .08$ & \\
\hline$A W-10 B$ & an TDS & $92<$ & $\leqslant$ & $2.24 \mathrm{E}-08<$ & $3.28 E-09$ & & $5.50 E-\infty)>$ & & AW-106 & all TOS & $92 \mid<$ & $2.24 E-06<$ & $2.30 E-05$ & $6>$ \\
\hline sY-103 & antos & $108=$ & 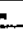 & $3.32 E-06,>$ & 3.75E- 94 & & $1.00 \mathrm{E}-0 \mathrm{~S}>$ & & sY.103 & All TDS & $106 \mid=$ & $3.32 E-0.05$ & $4.90 E-01$ & 1). \\
\hline $3 Y \cdot 101$ & All TOS & sor15, & 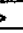 & $1.40 \mathrm{E}-04$ & $1.90 \mathrm{E}-05$ & & $4.30 E-03<$ & $\leq$ & SY-101 & all TDS & $801 />$ & t.40E-04 $>$ & $5.60 E-02$ & $21>$ \\
\hline & & & & & & & & & & & & & & \\
\hline Int ops-Ne & ed-And & No. eventis & & Mudian freq. & Median cons. & & Ex, Rlak & & Int ops-Non re & Yod-Tox & No. events & Madian freq. & Medien cons. & \\
\hline$A N-107$ & all TDS & 100 & $E$ & $1.40 E-05=$ & $2.75 E+10$ & & \begin{tabular}{|r|}
5.098 .06 - \\
\end{tabular} & $=$ & AN-107 & aUTSS & 100 & $1.48 E-05:=$ & 5.03E-00 & a. \\
\hline AW-106 & all TDS & $104=$ & & 3.07E-00: & $8.92 E-10$ & & $5,44 E-09=$ & & AW-10B & WTOS & 104 & $3,07 E-09=$ & $9.67 E-06$ & a. $=$ \\
\hline $5 Y-103$ & all TOS & $105=$ & & $2.45 E-05=$ & $2.18 E-\alpha$ & 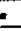 & $3.33 E-06=$ & $=$ & SY.109 & ant TOS & 106 & $2.45 \mathrm{E}-0.5=$ & 4.90E-01 & 1. \\
\hline SY-101 & alf TDS & 388. & $=$ & $1.23 E-04=\pi$ & $1.16 E-05$ & & $4,74 E-\infty 3=$ & & sY-101 & all TOS & $586:-1$ & 1.23E-04 = & $3.08 \mathrm{E}-02$ & 2 \\
\hline & & & & & & & & & & & & & & \\
\hline & & & & & & & & & & & & & & \\
\hline & & & & & & & & & & & & & & \\
\hline & & & & & & & & & & & & & & \\
\hline & & & & & & & & & & & & & & \\
\hline
\end{tabular}




\begin{tabular}{|c|c|c|c|c|c|c|c|c|c|c|c|c|c|c|c|}
\hline $\operatorname{lint} \operatorname{cps} \theta \cdot \mathrm{N}$ & inc. Rad & No. oventa & & Median treq. & & Moclan cons. & & Ex. A A & & Int ops- Non & ince-Tax & No. events & Median treq. & Modion cons. & \\
\hline $\mathrm{AN}-107$ & All TOS & 100 & $=$ & $1.46 \mathrm{E}-05$ & $=$ & $2.7 \mathrm{EE} \cdot 10$ & & $5,89 E-05 \mid=$ & & AN-107 & at TOS & $100 \mid=$ & $1.46 \mathrm{E}-05$ & $5.03 E-06$ & si= \\
\hline AW-108 & ali TOS & 104 & & 3.07E.08 & 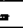 & 8.92E-10 & & $5.44 E-0 \theta=$ & & AW- 108 & sll TDS & $104:=$ & 3.07E-08 & $9.67 E-06$ & $\theta=$ \\
\hline $5 Y \cdot 103$ & all TDS & 105 & & $2.45 E-05$ & & $2.18 \mathrm{E} \cdot 0 \mathrm{~A}=$ & 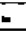 & $3.33 \mathrm{E}+0 \mathrm{5}=$ & & SY-103 & all TDS & $105=$ & $2.45 \mathrm{E}-05$ & 4.90E-01: & $t=x$ \\
\hline SY-101. & all TDS & 580 & & 1.24E-04 & & $1.18 E-05$ & & $4.74 E-\infty 3=$ & & SY.101 & at TOS & $508:=$ & $1.24 \mathrm{E}-04=$ & $3.08 \mathrm{E} \cdot 0 \mathrm{Q}$ & $2 x=$ \\
\hline & & & & & & & & & & & & & & & \\
\hline Int Ops-Le & I red- Rad & No. ovents & & Median freq. & & Median cons. & & Ex, Riak & & $\ln 1$ Opa-10x & al red. Tox & No. events & $\begin{array}{l}\text { Median freq. } \\
\end{array}$ & Medlan oons. & \\
\hline AN-107 & all TDS & 100 & & $1.48 E-05$ & - & $2.75 \mathrm{E}-10$ & $a$ & 5.02E-05 = & & $A N=107$ & all TDS & $100=$ & \begin{tabular}{|c|}
$1.40 E-05$ \\
\end{tabular} & 5.03E.06 & $B=$ \\
\hline AW-108 & all TDS & 100 & & $2.06 \mathrm{E}-\infty \mathrm{s}$ & & $4.83 E-07$ & 2 & $1.02 \mathrm{E}-089$ & & AW-106 & al TDS & $1001>$ & $2.80 E+08$ & $3.10 E-\infty 3$ & 3i) \\
\hline$s Y=103$ & autos & 122 & & $6.12 E-05$ & & 8.56E.05 & $<$ & $1.0 B E-05 \mid<$ & & $3 Y-103$ & aU TOS & $1221>$ & $6.12 \mathrm{E}-05,2$ & $3.13 E-01$ & it \\
\hline SY-101 & all TOS & 588 & & $1.24 \mathrm{E} \cdot 04$ & & $1.16 \mathrm{E}-0.5$ & - & $4.74 \mathrm{E}-00 \mathrm{~m}$ & & sY-101 & all TDS & 588. & $1.24 E-04=$ & $3.006-02$ & $=$ \\
\hline & & & & & & & & & & & & & & & \\
\hline Int opg-Le & I inc - Rad & No, eventa & & Median freq. & & Modlan cons. & & Ex. Rink & & Int Ops- LaOA & al inc-Tox & No. events & Median freq. & Median cons. & \\
\hline$A N-107$ & all TDS & 100 & & $1.46 \mathrm{E}-05$ & & $2.75 E \cdot 10=$ & & $5.83 \mathrm{E}-05$ & & AN-10? & all TDS & $100=$ & $1.46 \mathrm{E}-05$ & S.003E-08 & 5 \\
\hline AW. 106 & all TDS & 100 & & $2.42 \mathrm{E}-\mathrm{aB}$ & & 8.e7E-11 & & 1.60E-07> & & AW: 106 & all TDS & $100 k$ & $2.42 E-08$ & $2.12 E-06$ & 32 \\
\hline $5 Y-103$ & ant TDS & 105 & & $2.45 E-05$ & & $2.18 E-04=$ & & $3.34 E-06$. & & $s Y-103$ & All Tos & $105=$ & $2.45 \mathrm{E}-05=$ & 6.30E.01: & 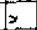 \\
\hline s $\gamma=101$ & ail TDS & $58 \mathrm{e}$ & & $1.24 E-04$ & & $1.16 \mathrm{E}-0 \mathrm{~S}=$ & $=$ & $4.74 E-\infty 9 \mathrm{n}$ & & SY-101 & all TDS & $580=$ & $1.24 E-04=$ & $3.08 E-09$ & $=$ \\
\hline & & & & & & & & & & & & & & & \\
\hline Int ops-GK & al red-fad & No. avents & & Median frea. & & Medjan oone. & & Ex. Alak & & Int ops- $\mathrm{alat}$ & bail red-Tox & No. event & Median treq. & Median cone. & \\
\hline$A \mathrm{~N}-107$ & an tos & 100 & & $1.46 \mathrm{E}-05$ & & $2,75 \mathrm{E}-10$ & & 5.75E-05 $\mathrm{k}$ & & AN. 107 & all Tos & $100=$ & 1.A8E-05 $=$ & \begin{tabular}{|l|}
$5.03 E-06$ \\
\end{tabular} & $=$ \\
\hline AW-108 & all TDS & 78 & & $7.63 E-06$ & & $6.08 \mathrm{E}-001>$ & & $4.30 E-00>>$ & & $A W-10 B$ & all Tos & $76 \mathrm{~K}$ & $7.63 E-08 \mid>$ & $1.34 \mathrm{E}+04$ & 1 \\
\hline SY-103 & all TOS & 95 & & $2.68 \mathrm{E}+0 \mathrm{~s}$ & & 2.95E-04 $>$ & & $1.13 E-04>$ & & SY-103 & all TOS & $95<$ & $2.68 \mathrm{E}-05>$ & $9.50 \mathrm{E}-011 \mathrm{z}$ & $1>$ \\
\hline SY-101 & att TDS & 589 & & 1.23E-04 & & $8,16 E \cdot 0.05$ & $=$ & $4.73 \mathrm{E}-031=$ & & $S Y+101$ & al TOS & $508, \pi$ & $1.23 \mathrm{E}-04=$ & $3.08 E-00$ & $=$ \\
\hline & & & & & & & & & & & & & & & \\
\hline Int ops-G & al inc- Fad & No. events & & Median treq. & & Modlan cons. & & Ex. Risk & & int ops-Gloo & Sed ine - Tox & No. Oventi & Median freq. & Medlan conna. & \\
\hline AN-107 & all TOS & $100=$ & & 1.48E-05- & & $2.75 E-40$ & & 3.98E-05> & & AN.107 & att TOS & $100=$ & $1.46 \mathrm{E}-05$ & $5.03 \mathrm{E}-08$ & $=$ \\
\hline$A W-108$ & lall Tos & 112 ? & & $\begin{array}{c}1.43 E-06 \\
\end{array}$ & & 6.90E-07)> & & $1.87 E-00>$ & & AW-108 & All TDS & $112>$ & $1.43 E-06<$ & 1.68E-OR & \\
\hline SY-103 & AHTDS & $106=$ & & $3.32 \mathrm{E}-0 \mathrm{~S}$ & & $5.75 \mathrm{E}-0.06$ & & 1.55E-04is & & sY-103 & all TOS & 108:- & $3.32 \mathrm{E}-0 \mathrm{~s}>$ & $1.56 \mathrm{E}+\infty 0$ & $>$ \\
\hline SY-101 & ad TDS & 596:> & & $1.4 \mathrm{BE}-04 \mid>$ & & $2.48 E-05>$ & & $4.71 \mathrm{E} .02>$ & & SY.101 & Ail TDS & $5961>$ & $1.46 \mathrm{E}-04>$ & $5.49 E-02$ & $>$ \\
\hline & & & & & & & & & & & & & & & \\
\hline Int 0op-Ex & ink inc- Pa $^{d}$ & No. events & & Medinn treq. & & Median cons. & & Ex. Alsk & & $\ln 1$ ops- Ex-to & ank inc - Tox & No. events & Median frect. & Median cons. & \\
\hline AN-107 & all TDS & 100 & & $1.46 \mathrm{E}-05$ & & $2.75 E-10$ & & 5.87E-0. $=$ & & AN-107 & AI TOS & $100=$ & $1.4 \mathrm{gE}-08=$ & $3.03 E-06=$ & $=$ \\
\hline$A W-106$ & all TDS & $104=$ & & $3.07 \mathrm{E}-06$ & & a. $.92 E-10=$ & & $5,44 E-09=$ & & AW- 100 & aH TDS & $104=$ & 3.07E-06:- & 9.67E-08 $=$ & \pm \\
\hline$S Y-103$ & all TDS & $106=$ & & $2.45 E-05=$ & & $2.10 \mathrm{E}-04=$ & & $3.33 E-05 \mid \approx$ & & $5 \gamma-103$ & all TDS & $105=$ & $2.45 E-05$ & $4.80 E-01$ & $=$ \\
\hline$s Y-101$ & all TOS & $580=$ & & $1.20 \mathrm{E}-04$ & & $1.16 \mathrm{E}-05=$ & & $4.74 \mathrm{E}-\infty \mathrm{S}=$ & & sY-101 & all TDS & 588 & $1.20 E-04:<$ & $3.00 \mathrm{E}-02$ & $=$ \\
\hline & & & & & & & & & & & & & & & \\
\hline $\operatorname{lgn} \operatorname{Cans}$ & In-tank - Rad & No. events & & Median treq. & & Median cons. & & Ex, Risk & & Itan. Cirl Set- & In-tank-Tax & No. events & \begin{tabular}{|l|} 
Median treq. \\
\end{tabular} & Median cons. & \\
\hline $\mathrm{AN}-107$ & sll TDS & $100=$ & & $1.486 .05=$ & & $2,75 E-10$ & & 5.83E-08 > & & AN-10? & at TDS & $i \quad 100 \mid \mathrm{m}$ & $1.46 E-05 \mid=$ & \begin{tabular}{|r|}
$5.03 E-08$ \\
\end{tabular} & $=$ \\
\hline AW-108 & all TDS & 104 . & & $4.97 E-00>$ & & $8.92 E+10=$ & & $4.48 E-08>$ & & $A W-10 B$ & all TDS & $104=$ & 4.97E-08 > & $1.23 \mathrm{E}-05$ & $>$ \\
\hline$s Y-103$ & all TDS & $105=$ & & $2.45 E-05=$ & & $2.18 E-04$ & & $1.60 E-04>$ & & $5 Y .103$ & all TDS & $105 \pi$ & $2.45 E-05$ & $6.86 \mathrm{E}-01$ & \\
\hline$\$ Y \cdot 101$ & all TDS & $588=$ & & $1.26 \mathrm{E}-0 \mathrm{~s})>$ & & $1.18 E-05=$ & 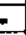 & $4.77 \mathrm{E}-03 \pi$ & & SY-101 & ail TDS & $580=$ & 1.28E.04 > & $3.08 \mathrm{E}-02$ & $=$ \\
\hline & & & & & & & & & & & & & & & \\
\hline $\operatorname{lgn} . \mathrm{CtrIS}$ & Ex-tank. Rad & No. menis & & Median freq. & & Modian cons. & & Ex. Figk & & Ign. Cirl Set- & Ex-tank . Tox & No. events & Median freq. & Median cons. & \\
\hline AN- 107 & all TDS & $100=$ & & $1.46 \mathrm{E}-05=$ & & $2.75 E-10=$ & 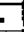 & $5.79 \mathrm{E}-05 \mid=$ & & AN-107 & Tall TDS & $1001=$ & $1,46 E-05$ & $5.03 E-\infty 6$ & \\
\hline$A W-10 B$ & ail TOS & $104=$ & & $4.97 \mathrm{E}-06$ & & $8.92 E \cdot 10=$ & & $4.49 \mathrm{E} \cdot 0 \mathrm{O})$ & & AW-106 & all TOS & tou. & $4.97 \mathrm{E}-08>$ & $1.23 \mathrm{E}-05$ & \\
\hline$\$ Y-103$ & all TDS & $105=$ & & $2.45 E-05=$ & & $2.18 \mathrm{E}-04=$ & & $3.33 \mathrm{E} \cdot 05=$ & & $\$ S Y+103$ & al TOS & $1051=$ & $2.45 E-05=$ & $4.90 \mathrm{E}-01$ & $=$ \\
\hline SY-101 & Ial TOS & $5881=$ & & $1.24 E-04=$ & & $1.16 E-05=$ & & $4.75 \mathrm{E}-03=$ & & SY.104 & all TDS & 588 & $1.28 \mathrm{E}-04>$ & $3.08 \mathrm{E} \cdot 02$ & $=$ \\
\hline & & & & & & & & & & & & & & & \\
\hline $\operatorname{lgn}$ Cor S & InEx-tank - P & No. events & & Medlen trea. & & Median cons. & & Ex. Risk ... & & $\lg$. Cos Set & WVEx-lsnk - r & No. ovents & Madian trea. & Medien cane. & \\
\hline $\mathrm{AN}-107$ & a: TDS & 100:- & & $1,46 \mathrm{E}-05$ & & $2.75 E-10$ & & 5.80E-05|> & & AN-107 & ail Tos & $100=$ & 1.46E-05.m & 5.03E.08 & $=$ \\
\hline$A W-106$ & ail TDS & $104=$ & & $4.97 \mathrm{E}-08>$ & & B.92E-10 & & $4.49 \mathrm{E}-08$ & & $A W \cdot 106$ & all TDS & $104=$ & 4.97E-06> & $1,23 \mathrm{E}-05$ & \\
\hline SY.103 & ail TDS & $105=$ & & $2,45 \mathrm{E}-05$ & & $2.18 \mathrm{E}-04$ & & $1.60 \mathrm{E}-04>$ & & $s Y+103$ & all TDS & $105=$ & $2.45 E-05=$ & $6,665-01$ & \\
\hline SY-10T & all TDS & $588=$ & & $1.26 E-04>$ & & $1.18 E+05$ & & $4.78 \mathrm{E}-00=$ & & SY-10: & all TDS & $580=$ & $1,24 E-04=$ & $3.08 \mathrm{E}-02$ & $=$ \\
\hline & & & & & & & & & & & & & & & \\
\hline Density rat & Liq 10 S.Sol - & No. eventa & & Median freq. & & Medlan cons. & & Ex. Alak & & Denalty ratlo & Liq to S.Sol. ? & No. events & Madian froq. & Median cons. & \\
\hline$A N-107$ & aA TDS & $1162>$ & & 1.21E-04 $>$ & & $1,05 E-06>$ & & $1,83 E-\infty 3>$ & & AN-107 & ax TDS & $1162 \mid>$ & $1.21 \mathrm{E}-04 \mid>$ & $2.17 \mathrm{E}-02,3$ & \\
\hline$A W-106$ & all TOS & $1094>$ & & $1.52 \mathrm{E}-041>$ & & $2.15 \mathrm{E}-08>$ & & $7.99 \mathrm{E}-07 \mid>$ & & $A W=108$ & II TDS & 1094 s & $1.52 E-04$ & $4.54 \mathrm{E}-02 \mathrm{I}=$ & \\
\hline SY-109 & all TDS & 1182 & & $1.38 E-04 \mid>$ & & $1.23 \mathrm{E}-04<$ & & $4.21 E-04>$ & & sY-103 & all TDS & $1182>$ & $1.38 E-04>$ & $2,41 \mathrm{E}-01$ & \\
\hline SY-10t & all TDS & 1831> & & $1.71 \mathrm{E}-04>$ & & $2.55 \mathrm{E}-0<>$ & & $1.74 \mathrm{E}-02 / \mathrm{s}$ & & $5 Y \cdot 101$ & aH TDS & $580=$ & $1.26 \mathrm{E}-04>$ & $3.00 \mathrm{E}-02$ & \\
\hline & & & & & & & & & & & & & & & \\
\hline Denaity rat & Liq bo l.sot - Fa & No. evente & & Median freq. & & Median cons. & & Ex. Alak & & Deneity ratio & $\mathrm{Laq}$ to t.sol - Td & No. DNents & \begin{tabular}{|l|} 
Medlen freq. \\
\end{tabular} & Median cona. & \\
\hline AN-107 & aif TDS & $100=$ & & t.48E-06 & & \begin{tabular}{|c|}
$2.75 \mathrm{E}-10$ \\
\end{tabular} & & $\quad 3.83 E-\infty s_{1 x}$ & & $A N=107$ & aATDS & $100=$ & $1.46 \mathrm{E}-05=$ & 5.03E-09 & \\
\hline$A W-106$ & all TOS & $104=$ & & $3.07 E-08=$ & & a.92E-10= & & $5.44 E-08=$ & & AW-108 & $\triangle 4$ TDS & $104 \mid=$ & $3.07 \mathrm{E}-06=$ & $9,67 \mathrm{E}-08$ & \\
\hline $5 Y .703$ & all TOS & $105 \mid=$ & & $2.45 \mathrm{E}-05)=$ & & $2.02 E-04<$ & & $3.09 E-05 \mid<$ & & $S Y-103$ & att TDS & $105=$ & $2.45 \mathrm{E}-05 \mathrm{I}_{x}$ & $4.55 \mathrm{E}-01$ & \\
\hline SY +101 & all TOS & $508=$ & & $1.24 E \cdot 04 \mathrm{a}$ & & $1.15 \mathrm{E}-06=$ & & $4.74 \mathrm{E}-03=$ & & SY-101 & all TDS & $580=$ & 1.24E-04 & $3.08 \mathrm{E}-02$ & \\
\hline & & & & & & & & & & & & & & & \\
\hline Gas genen & on ratios.s.sol- & No. events & & Medkan freq. & & Median oons. & & \begin{tabular}{|l|l|} 
Ex. Rligk \\
\end{tabular} & & Gas goneral & lon rate S.sot- & \begin{tabular}{|l|l} 
No. events \\
\end{tabular} & Median treq. & Median cons. & \\
\hline AN-107 & all TDS & $100=$ & & $1,48 E-05$ & & $2.75 \mathrm{E}-10$ & & 3.606-04]> & & $A N-107$ & AHTOS & $1001 \mathrm{~m}$ & $1.46 \mathrm{E}-05=$ & $5.03 E-06$ & $=$ \\
\hline$A W=106$ & all TDS & $88<$ & & $6.78 E-\infty 6$ & & 8.46E-07 > & & $6.51 \mathrm{E} \cdot 0 \mathrm{~B} />$ & & $A W \cdot 108$ & au TOS & $88<$ & $6.78 E-061>$ & 6.96E-03) & $>$ \\
\hline $5 Y-103$ & all TOS & $102 i<$ & & $4.56 \mathrm{E}-05>$ & & $1.15 E-04<5$ & & $2.10 E-04>$ & & sY-103 & all TDS & $102<$ & $4.56 E-051>$ & $3.81 E-01$ & \\
\hline $5 Y \cdot 101$ & all TDS & $580=$ & & $+200-04>$ & & $1.16 E-0 S=$ & & $4.72 E-02>$ & & SY-101 & all TOS & $586=$ & $1.24 \mathrm{E}-04$ & $3,08 E-02$ & $=$ \\
\hline & & & & & & & & & & & & & & & \\
\hline Gas ganer & on rale t.aol. & No. events & & Modien freq. & & Median cons. & & Ex. Alibix & & Gas gonerat & On rate i.sol- & No. avente & Median treag. & Madian cons. & \\
\hline$A N-107$ & all TDS & $3<$ & & $2.90 \mathrm{E}-013$ & & 7.87E-OQ $>$ & & 1.73E-05|< & & $A N-107$ & all TOS & $3<$ & $2.90 E \cdot 01>$ & $3.71 E+00$ & 2 \\
\hline$A W-10 B$ & all TDS & $16<$ & & $1.82 E-05>$ & & $7.96 \mathrm{E}-04>$ & & $1.90 \mathrm{E}-06)>$ & & AW.106 & All TOS & $16<$ & $1.82 E-05>$ & $1.37 \mathrm{E}+01$ & $\geq$ \\
\hline $3 Y-103$ & all TOS & $39 .<$ & & $7.32 E-06<$ & & $2.67 \mathrm{E} \cdot 04>$ & & $4.25 E-04>$ & & sY-103 & all TDS & $39<$ & $7.32 E-06<$ & 3.94E-01 & $\leq$ \\
\hline$y-301$ & all TOS & $184<$ & & $1.58 \mathrm{E}-04)$ & & $2.43 \mathrm{E}-04>$ & & $7.09 \mathrm{E}-00)>$ & & $s Y-101$ & at TOS & $104<$ & $1.22 E-04<$ & $3.09 \mathrm{E}-09$ & \\
\hline & & & & & & & & & & & & & & & \\
\hline & & & & & & & & & & & & & & & \\
\hline & & & & & & & & & & & & & & & \\
\hline & & & & & & & & & & & & & & & \\
\hline & & & & & & & & & & & & $i$ & & & \\
\hline
\end{tabular}




\begin{tabular}{|c|c|c|c|c|c|c|c|c|c|c|c|c|c|c|c|}
\hline \multicolumn{2}{|c|}{ Tank fail preses red.- Pitd } & \multirow{2}{*}{\begin{tabular}{|r|} 
No. events \\
100
\end{tabular}} & & Median frea. & & Medien conit. & Ex. Rhak & & Tank tail pres & red. - Tox & \begin{tabular}{|l|l|} 
Na. ovente \\
\end{tabular} & Medlan freq. & & Madian $\infty$ ons. & \\
\hline$A N-107$ & All TOS & & & & & \begin{tabular}{|r|}
$2.75 E-10$ \\
\end{tabular} & 5.00E-05 & & AN-107 & all TOS & $100=$ & $1.48 E-05$ & $=$ & $5.03 \mathrm{E}-\infty)$ & 1: \\
\hline & Onaite Aed: : & 2.2 & & $1.00 E-05=$ & & $2.30 \mathrm{E}-11=$ & $4,60 \mathrm{E}-08$ & $=$ & & Onaite Tox: & $82 .=$ & $1.00 \mathrm{E}-0 \mathrm{~B}$ & $=$ & $5.20 E-07$ & $=$ \\
\hline & Onsitte Red: & 3 & 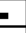 & 2. $.0 E-01=$ & & $7.67 \mathrm{E} .08$ & 1.73E-03 & 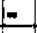 & & Onpits Tox: & $3:=$ & 2.80E-01: & $=$ & $3.71 E+\infty 0=$ & $=$ \\
\hline & Oneile Rad: & 15 & $a$ & 3.83E-01 $=$ & & 6.39E-05 & $3.97 E-09 ;$ & $=$ & & Onaits Tox: & 15 & 3.83E-01: & $=$ & $3.24 E+011=$ & $=$ \\
\hline$A W=106$ & all TOS & 104 & \pm & 3.07E-00 & & 0.92E.10 & $8.53 E-10$ & $\leq$ & AW-10B & all TDS & $104=$ & $3.07 \mathrm{E}-06$ & $=$ & $9.67 \mathrm{E}-00 \mathrm{e}=$ & $\equiv$ \\
\hline & Onaite Rad: & 84 & & 2.38E- -06 & & $4.60 \mathrm{E}-12=$ & B.17E-11 & $=$ & & Onatite Tox: & $84:=$ & $2.39 \mathrm{E}-06$ & $=$ & 2.3BE-OQ $=$ & $=$ \\
\hline & Oraition rad: & of & 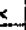 & $2.44 E-06)>$ & & $1.50 E-05 s$ & $2,05 E-08$ & $<$ & & Onaite Tox: & al $\leq$ & $2,44 \mathrm{E}-05$ & $\geq$ & $1.33 \mathrm{E}-01<$ & $<$ \\
\hline & Onaste Rad: & 32 & 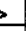 & $2.69 E \cdot 06 \mid>$ & & $0.65 E-06$ ? & $2.29 E-07$ & 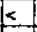 & & Onaite Tox: & $32>$ & $2.86 E-08$ & $>$ & $8,60 \mathrm{E}-02<<$ & $<$ \\
\hline SY.100 & a tos & 105 & & $2.46 E \cdot 06=$ & 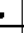 & $3.18 E-04>$ & 3.29E- 95 & $\leq$ & SY-103 & all TDS & 105. $=$ & 2.45E.05. & $=$ & 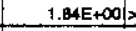 & \\
\hline & Onaite Rad: & 15 & & $1,53 \mathrm{E}-0.9=$ & - & Q. $19 \mathrm{E}-10$ & 4.365 .08 & $=$ & & Onsile Tox: & $15=$ & $1,59 \mathrm{E}-\infty]^{2}$ & $=$ & 4.37E-08|= & $i=$ \\
\hline & Onaits Rad: & 17 & $\leq$ & $5.04 E-08$ & 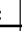 & $2.88 E-04)>$ & $1.60 E-04$ & $<$ & & Onaite Tox: & $171<$ & $5.04 E-06$ & $\leqslant$ & $2.41 \mathrm{E}-01 \mathrm{~K}$ & $<$ \\
\hline & Onaite Rad: & 79 & $>$ & 3.47E-05 & 5 & 1.13E-03>> & $1.32 E-\infty$ & $<$ & & Onalte Tox: & $73 \mid>$ & $3.47 E-05$ & $\leq$ & 3.19E+00: & K \\
\hline sY-101 & allos & 588 & 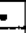 & $1.24 \mathrm{E}-04$ & 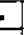 & $9.95 E-08<$ & $4.805-003$ & $\geq$ & $s Y-101$ & A\#TOS & 508 . & $1.24 E-04=$ & $=$ & $3.08 \mathrm{E}-\infty 2=$ & $=$ \\
\hline & Onsilo Rad: & 275 & \pm & 4.70E-05:- & 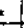 & $1.59 \mathrm{E}-009=$ & 9.57E-07 $=$ & $=$ & & Onaite Tax: & $275=$ & $4,70 \mathrm{E}-0 \mathrm{OS}$ & $=$ & $4.41 \mathrm{E}-05=$ & $\pi$ \\
\hline & Onaile Aed: & 104 & $\leq$ & $\mid .22 E+04<$ & $\varepsilon$ & $3.97 E-043$ & 8.3AE-03) & $>$ & & Onnile Tox: & $184=$ & $1.59 \mathrm{E}-04$ & $=$ & 4.33E-01 = & $=$ \\
\hline & Oneits Red: & 2009 & 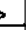 & $2.99 E-04<$ & & $1.00 E-03 \mid>$ & $3.16 \mathrm{E}-02$ & $<$ & & Onalte Tox: & $129=$ & $3.40 \mathrm{E}-04$ & $=$ & $5.58 E+\infty=$ & $=$ \\
\hline & & & & & & & & 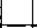 & & & & & & & \\
\hline WIE Opa ino & & No. events & & Medlan frea. & & Median oons & Ex. Riak & & WIE Opa inc & & No. events & Median freq. & & Mectien sons & \\
\hline AN-107 & aU TOS & 129 & 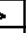 & \begin{tabular}{|c|}
$2.07 E-05 \mid>$ \\
\end{tabular} & 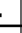 & $7.48 \mathrm{E} \cdot 10 \mid>$ & $8.405-05$ & $\geqslant$ & $A N-107$ & all TDS & $\mid 128>$ & 2.07E-05, & 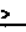 & 1.21E-0s; & $\Rightarrow$ \\
\hline & WIEquip (all & 29 & 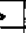 & 1.07E-02), & & $2.98 E .098$ & 5.68E-06: & $\geq$ & & WIEquip (all) & $29 .>$ & $1.07 E \cdot 02: 3$ & $\geq$ & Q.6AE- $-\infty ;>$ & 3 \\
\hline$A W=106$ & ail TOS & 107 & & $3.67 \mathrm{E}-00$ ) & & $1.01 \mathrm{E} \cdot 00 \mathrm{~s}>$ & $1.01 E+003>$ & $\geq$ & AW-10B & \&ע TDS & $1071>$ & $3.67 \mathrm{E}-06$ & $\geq$ & $9.87 \mathrm{E}-0 \mathrm{a}$ & $\pi$ \\
\hline & WIEquip (nil) & 3 & 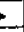 & $7.665-09$ & & $9.49 E-00$ ? & $1,53 E-06$ & $>$ & & WIEquip (all & $3 \mid 3$ & 7.50E-03) & $\geq$ & $1.28 E-\infty 2 ;$ & $>$ \\
\hline $5 Y-103$ & ail TDS & 114 & 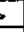 & $3.15 E \cdot 05$ > & & $2.55 E-04>$ & $9.62 E-04$; & $\geq$ & $5 Y .100$ & \begin{tabular}{|l|} 
II TDS \\
\end{tabular} & $111 \times$ & 3.15E.05, & $\geq$ & 8.48E-01) & 2 \\
\hline & WiEquip (a)|' & 9: & 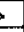 & $1.67 \mathrm{E}-02>$ & & $7.81 \mathrm{E}-04>$ & $9.49 E-0<$; & 2 & & WIEquip \{ (n: & 9) & 1.67E-02)> & $>$ & $1.73 E_{+}+02,>$ & $>$ \\
\hline SY-101 & all TDS & 607 & 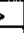 & 1.27E-04> & & 1.10E-05 K & $3.63 \mathrm{E}-\infty \mathrm{B}$ & $<$ & $5 Y-101$ & ATDS & 607/s & $1.27 \mathrm{E} 0 \mathrm{C}$, & $\geq$ & $3.41 \mathrm{E}-02,>$ & $\geq$ \\
\hline & WiEquip (ail) & 18 & 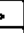 & $3.98 \mathrm{E}-03>$ & & $5.62 E-07 \mid>$ & $2.05 E-041>$ & $\geq$ & & WIEquip \{ail\}? & $10 \mid>$ & $3.8 \mathrm{BE}-0 \mathrm{O}$ & 2 & $1.37 E+00>$ & 7 \\
\hline & & & & & & & & & & & & & & & \\
\hline WIE Opa inc- & ign.ctel & No. events & & Median freq. & & Median cons & Ex. Alsk & & WIE Oos inc-1 & ten,ctr & No. eventu & Median frea. & & Modian conn & \\
\hline AN-107 & al Tos & 848 & 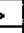 & \begin{tabular}{|c|c|}
$0.58 \mathrm{E}-03 \mid$ & $>$ \\
\end{tabular} & & \begin{tabular}{r|r|}
$7.55 E \cdot 07$ & \\
\end{tabular} & 1.51E-04, & $>$ & $A N \cdot 107$ & all TDS & 048 $?$ & 6. $18 \mathrm{E}-\infty)$ ? & 3 & 9.64E-01)> & 2 \\
\hline & WIEquip (all' & 548 & 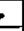 & $8.50 \mathrm{E}-\infty) \leq$ & & $7,55 \mathrm{E}-07$ & $1.51 \mathrm{E}-04 / 3$ & $>$ & & WtEquip \{ail - & $648>$ & 8.68E-03 $<$ & $\leqslant$ & 9.84E-01)> & 2 \\
\hline$A W-100$ & .11508 & 854: & 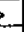 & $0.32 E-\infty 9$ & & $1.97 \mathrm{E}-08>$ & $2,41 \mathrm{E}-04$ & $>$ & AW-108 & all TDS & $254>$ & $6.32 \mathrm{E}-00$, & $\geqslant$ & $2.20 \mathrm{E}+00 \mid>$ & $\geq$ \\
\hline & WiEquip (all' & 750 & 2 & $0.32 E-09 \leq$ & & $1.07 E-06>$ & $2.41 \mathrm{E}-04>$ & $\geq$ & & WIEquip (all ' & 654:> & 8.32E- 03 & $\leq$ & $2.20 E+\infty 0$ & $>$ \\
\hline SY.103 & all TOS & 835 & 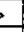 & $7.08 E-03>$ & & 1.0BE.04< & $2.55 \mathrm{E}-\infty 2$ & 2 & SY-103 & all TDS & $\Delta 38>$ & $7.09 \mathrm{E}-03$ ? & $?$ & $1.07 E+01>$ & $>$ \\
\hline & WIEquip (a)1 & $730:=$ & & $7.09 E-\infty)<$ & 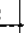 & $1.005-04<$ & $2.55 \mathrm{E} \cdot 02$ & $>$ & & WIEquip (all ? & 8955 $\geq$ & $7.09 \varepsilon-03$ [S & $<$ & $1.07 E+01<$ & $\leq$ \\
\hline SY-101 & all ros & 1255 & 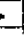 & $1.13 E-\infty)>$ & & $2.79 \mathrm{E}-05>$ & $2.44 \mathrm{E} \cdot 0 \mathrm{O}$ & $\alpha$ & sY-101 & all TOS & $1255>$ & $1.13 E-03$, & $\geq$ & 6.08E-01? & $?$ \\
\hline & WIEquip (all - & 687 & & $1,13 E-\infty)<$ & 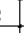 & $2.73 \mathrm{E}-05$ > & 2.4.4E-03:2 & $\geq$ & & WIEquip (aik 1 & $687>$ & $8.44 E+03>$ & $\geq$ & $7.00 E+00>$ & $>$ \\
\hline & & & & & & & & & & & & & & & \\
\hline WIE Ops ine- & aquip dia & No. events & & Median treq. & & Median cons & Ex. Alak & & WIE Ope inc-A & qquip din & No. went & No. deftao. & & No. detan. & \\
\hline AN-107 & All TDS & 129 & 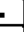 & 2.87E-05i= & & B.47E-10< & 6.22E- 05 & $\leq$ & $\mathrm{AN}-107$ & AT TDS & $129=$ & $2.07 \mathrm{E}-05$ & $=$ & $1,21 \mathrm{E}-05=$ & $=$ \\
\hline & WiEquip (all- & 29 & $E$ & $1.07 E-02$ & & $2.08 \mathrm{E}-00 \mathrm{C}_{\mathrm{C}}$ & $3.86 \mathrm{E}-08$ & $\leq$ & & WIEquip (aill & $28=$ & $1.07 \mathrm{E}-02=$ & $E$ & $4.68 \mathrm{E}-03<$ & $\leq$ \\
\hline$A W-106$ & all TDS & $107=$ & 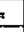 & $3.67 E \cdot 08$ & & $1.01 \mathrm{E}+09=$ & $8.71 E \cdot 09$ & $\leq$ & AW.108 & All TDS & $107=$ & $3.87 E-08=$ & 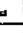 & $9.67 \mathrm{E}-06=$ & $=$ \\
\hline & WIEquip (all - & $3=$ & $=$ & $7,66 \mathrm{E}-0.09$ & & $8.84 E-08$. & $1,005 \cdot 08$ & $<$ & & WIEquip (all. & $3:=$ & 7.66E- $-03=$ & 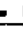 & Q.98E.00 < & $\leq$ \\
\hline Sy.103 & all TDS & $r+1=$ & 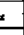 & 3.15E-05i= & & $2.43 \mathrm{E} .04<$ & $0.97 \mathrm{E}-04$ & $<$ & $5 Y-103$ & all TDS & $114=$ & $3.15 E-05=$ & $=$ & 8.48E-01 = & $=$ \\
\hline & WiEquip (ail) & 9 & 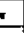 & $1,67 \mathrm{E}-02$ & & $5.46 E-04 k$ & 3.64E-04. & $\leq$ & & WIEquip (gat) & $99=$ & 1.67E- -02 & 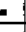 & $7.99 E+01<<$ & $\leq$ \\
\hline sY-101 & all ros & 607 & 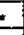 & $1,27 \mathrm{E}-04=$ & & $1.10 \mathrm{E}-05=$ & $3.61 \mathrm{E}-03$ & $=$ & SY-101 & AllTDS & $607 \mathrm{l}=$ & $1.27 \mathrm{E}-04=$ & 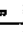 & $3.41 \mathrm{E} \cdot 02$ & - \\
\hline & WIEquip (all & 18 & 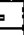 & $3.98 E-03=$ & 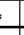 & $3.08 E-07<$ & $1.43 \mathrm{E}-04$ & $\leq$ & & WrEquip \{all & 10! & $3.88 E-09=$ & $E$ & $9.59 \mathrm{E}-0 \mathrm{t}$ < & $\leqslant$ \\
\hline & & & & & & & & & & & & & & & \\
\hline WIE Cps ine-e & equip len & No. ovenis & & Median freq. & & Median cons & Ex. Alax & & WIE Qpe ino-e & equip lan & No. eventa & No. dofleg. & & No. dalon. & \\
\hline$A N-107$ & all TDS & 128 & $=$ & 2.87E-0s & 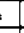 & $7.46 E-10=$ & \begin{tabular}{|c|}
$6.46 E-05$ \\
\end{tabular} & $E$ & $A N-107$ & nil TDS & $129=$ & 2.87E-05 & $E$ & 1.21E-05|w & $=$ \\
\hline & WIEquip (all & 28 & $x$ & 1.07E-08 $=$ & 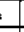 & $3.28 E-09>$ & $6.29 E-00$ & $\$$ & & WiEquip (ail' & $20=$ & $1.07 \mathrm{E}-02=$ & 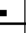 & $7.33 E-0,>$ & $>$ \\
\hline$A W=100$ & - 11 TOS & 107 , & 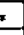 & $3.67 \mathrm{E}-06$ & & 1.01E-09 $=$ & $9.71 E-09$ & $\leq$ & AW. $10 \mathrm{~B}$ & all TOS & $107=$ & $3.67 \mathrm{E} \cdot 09$ & 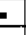 & $9.07 \mathrm{E} .06$ & 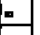 \\
\hline & Wiequip (ail - & 3. & $=$ & $7,6 B E-\infty)=$ & & B. $68 \mathrm{E}-00<$ & $1.41 \mathrm{E}-0 \mathrm{e}$ & $<$ & & WiEquip ast? & $3=$ & $7.86 \mathrm{E}-00$ & $E$ & $1,18 E-02<<$ & $\leq$ \\
\hline$S Y-103$ & all TDS & 114 : & $E$ & $3.15 \mathrm{E}-05$ & $E$ & $2.68 E-04$ & $1,09 \mathrm{E}-03$ & $>$ & SY-103 & all TOS & $114=$ & 3.15E-05: & $E$ & 6.48E-01 = & $=$ \\
\hline & WIEquip (ail - & 9 & $E$ & $1,67 E-02$ & 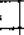 & 9.80E-04 > & $1,06 \mathrm{E}-03$ & $>$ & & WiEquip (a) ? & $9 \mid=$ & $1.67 \mathrm{E}-02$ & $E$ & $1.25 \mathrm{E}+02$ & $>$ \\
\hline SY-101 & 패 TD8 & 607 & $E$ & $1.27 \mathrm{E}-04 \mathrm{~A}$ & & $1.10 \mathrm{E}-0 \mathrm{~s}$ & $3.84 \mathrm{E}-03$ & $=$ & sY.101 & all TDS & $607=$ & $1.27 \mathrm{E}-04$ & $E$ & $3.41 E-02$ & 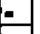 \\
\hline & WiEquip (all - & $19=$ & & $3.0 \mathrm{aE}-03=$ & & $3.986 .07 /<$ & $1.43 \mathrm{E}-0.4$ & 5 & & WIEquip (all' & $10 \mid=$ & $3.98 \mathrm{E}-003$ & 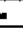 & $1.26 \mathrm{E}+00 \mathrm{~K}$ & $k$ \\
\hline & & & & & & & & & & & & & & & \\
\hline & & & & & & & & & & & & & & & \\
\hline & & & & & & & & & & & & & & & \\
\hline & & & & & & & & & & & & & & & \\
\hline & & & & & & & & & & & & & & & \\
\hline & & & & & & & & & & & & & & & \\
\hline & & & & & & & & & & & & & & & \\
\hline & & & & & & & & & & & & & & & \\
\hline & & & & & & & & & & & & & & & \\
\hline & & & & & & & & & & & & & & & \\
\hline & & & & & & & & & & & & & & & \\
\hline & & & & & & & & & & & & & & & \\
\hline & & & & & & & & & & & & & & & \\
\hline & & & & & & & & & & & & & & & \\
\hline & & & & & & & & & & & & & & & \\
\hline & & & & & & & & & & & & & & & \\
\hline & & & & & & & & & & & & & & & \\
\hline & & & & & & & & & & & & & & & \\
\hline & & & & & & & & & & & & & & & \\
\hline & & & & & & & & & & & & & & & \\
\hline & & & & & & & & & & & & & & & $i$ \\
\hline
\end{tabular}


This page intentionally left blank .

Att-B-10 
RPP-6888 REV 0

APPENDIX C

SST ACCEPTANCE TEST RESULTS 
RPP-6888 REV 0

This page intentionally left blank.

Att-C-ii 


\title{
RPP-6888 REV 0
}

\begin{abstract}
APPENDIX C
This Appendix provides the results of the SST acceptance testing. A strict comparison of the benchmark results to the trend analysis results does not consider the magnitude of the difference. For example, if the benchmark mean consequence value is $4.567 \mathrm{E}-05 \mathrm{~Sv}$ and the mean trend test analysis result is $4.566 \mathrm{E}-05$ $\mathrm{Sv}$, a comparison of this type would indicate, that by modifying a specific parameter, the consequences decreased; however, based on the conservatisms and uncertainties incorporated in the Analysis Tool, a better conclusion would be that there is no change. Therefore, to interpret the results, i.e., determine the change in the benchmark values due to changing specific parameters, the comparisons shown in the tables provided for each of the trend test analysis results are based $+/-10 \%$.
\end{abstract}




\section{RPP-6888 REV 0}

This page intentionally left blank.

Att-C-iv 


\begin{tabular}{|c|c|c|c|c|c|c|c|c|c|c|c|c|}
\hline \multicolumn{13}{|c|}{ SST ACceptance test resuits: } \\
\hline \multicolumn{13}{|c|}{ Seod: 90000000} \\
\hline \multicolumn{13}{|c|}{ Sample count: 1000} \\
\hline & & & & & & & & & & & & \\
\hline \multicolumn{2}{|c|}{ Benchmark - \%LFL } & & & & & & & $\% L F L$ & & $\%$ LL & & \\
\hline Tank & & No. events & & No. deffiag. & & No. deton. & & Mecian ireq. & & Medlan conc. & & \\
\hline \multirow[t]{4}{*}{ S.102 } & all sizes & 11877 & $B$ & 2116 & B & $\begin{array}{r}\text { 이 } \\
\end{array}$ & & 5.89E-05 & $\mathrm{B}$ & $\begin{array}{r}12.5 \\
\end{array}$ & $\mathrm{~B}$ & \\
\hline & smaill & 3957 & 是 & 204 & $\mathrm{~B}$ & & 8 & $5,47 E-04$ & 8 & 2.77 & 8 & \\
\hline & medium & 3960 & $\mathrm{~B}$ & 532 & $B$ & O. & 日 & $7.78 \mathrm{E}-05$ & $B$ & 17.1 & $B$ & \\
\hline & large. & 3960 & $\mathrm{E}$ & 1380 & $B$ & & $B$ & 4.47E-06 & 8 & 73 & $B$ & \\
\hline \multirow[t]{4}{*}{ U-111 } & ant & 12272 & $\theta$ & 716 & $\mathrm{~B}$ & OIE & B & $6.34 \mathrm{E}-05$ & $\mathbf{B}$ & 5.19 & 8 & \\
\hline & smail & 4088 & $B$ & 104 & B & 0. & 8 & $6.35 \mathrm{E}-0.4$ & $\mathrm{~B}$ & 1.04 & B & \\
\hline & medium & 4092 & $B$ & 304 & $B$ & 이토 & $B$ & $9.44 \mathrm{E}-0.5$ & E & 6.99 & $B$ & \\
\hline & large & 4092 & 8 & 308 & $B$ & $O E$ & $B$ & $4.86 E-06$ & $B$ & $29.8: 8$ & 8 & \\
\hline \multirow[t]{4}{*}{$T-203$} & all sizes & 11985 & $B$ & 1538 & $B$ & $84 . E$ & 8 & $5.20 \mathrm{E}-08$ & $B$ & $7.43 \mathrm{E}$ & 日 & \\
\hline & small & 3993 & $B$ & 12 & $B$ & $0 \mid \mathrm{E}$ & & 4.05E-05 & 8 & $1.95 \mid \mathrm{E}$ & $B$ & \\
\hline & medium & 3996 & $B$ & 184 & 8 & 이른 & $B$ & 7.27E-06 & B & $11.6 \mathrm{E}$ & $B$ & \\
\hline & large & 3996 & $\mathrm{e}$ & 1340 & $B$ & $84 \mathrm{E}$ & 8 & 4.35E-07 & $B$ & $56 \mathrm{E}$ & $B$ & \\
\hline \multirow[t]{4}{*}{$B-111$} & all sizes & 12034 & $B$ & $28 \mathrm{~B}$ & $B$ & 이 & $B$ & $3.45 E-05$ & $B$ & 1.63 & $B$ & \\
\hline & smail & 4010 & $B$ & 8 & $B$ & OfB & $B$ & $3.09 \mathrm{E}-04$ & $B$ & 0.451 & $\mathrm{~B}$ & \\
\hline & modium & 4012 & 8 & 68 & $B$ & 08 & $B$ & $5.32 \mathrm{E}-0.5$ & B & 2.53 & $B$ & \\
\hline & large & 4012 & B & 192 & $B$ & 0,1 & 8 & $2.70 E-06$ & $B$ & 11.7 & $B$ & \\
\hline WIE All & & 0 & $B$ & 0 & B & 018 & B & $0.00 \mathrm{E}+00$ & $B$ & & $B$ & \\
\hline & & & & & & & & & & & & \\
\hline & & & & & & & & & & & & \\
\hline S.Wesl p & ing - \%LFL & & & & & & & $\%$ LFL & & $\%$ LLL & & \\
\hline Tank & & No. events & & No. dellag. & & No. deton. & & Median irea. & & Median conc. & & \\
\hline S-102 & all skes & 11984 & $=$ & 1824 & $<$ & 0 & I & 3.73E-05 & $<$ & 11.4 & $<$ & \\
\hline $\mathrm{U}-111$ & all sizes & 11974 & $\leqslant$ & 560 & $\leq$ & $0=$ & $=$ & 4.37E-05 & $<$ & 4.31 & $<$ & \\
\hline T-203 & all sizes & 12236 & $>$ & 1488 & $<$ & $5 B<$ & & 4.17E-06 & $\leq$ & $6.56<$ & $\leq$ & \\
\hline$B-111$ & all sizes & 11913 & $\leqslant$ & 204 & $<$ & $0=$ & & $2.90 E-05$ & $<$ & 1.31 & $<$ & \\
\hline Waste V & d. - \%LFL & & & & & & & \begin{tabular}{|l|l|}
$\%$ LFL \\
\end{tabular} & & $\%$ LFL & & \\
\hline Tank & & No. events & & No. dellag. & & \begin{tabular}{|l|} 
No. deton. \\
\end{tabular} & & Median freq. & & Median conc. & & \\
\hline S-102 & ail sizes & 11913 & $=$ & 856 & $<$ & 0 & & $6.11 \mathrm{E}-05$ & 2 & 6.09 & $\leq$ & \\
\hline$(4-111$ & all sizes & $12199=$ & $=$ & 312 & $<$ & 이 = & $=$ & $6.34 \mathrm{E} \cdot 05$ & $=$ & 2.76 & $\leq$ & \\
\hline$T-203$ & all sizes & $11974=$ & $=$ & 636 & $<$ & $4 \mid<$ & $\leq$ & $5.91 E-061=$ & $\geq$ & 3.51 & $<$ & \\
\hline $\mathrm{B}-111$ & all sizes & 11877 & $<$ & 88 & $\leq$ & $0=$ & & $3.73 E-05$ & $\geq$ & 0.921 & $<$ & \\
\hline & & & & & & & & & & & & \\
\hline Intrusive & Alt $-\% \mathrm{LL}$ & & & & & & & $\%$ LLL & & $\%$ LFL & & \\
\hline Tank & & No. events & & No. deflag. & & No. deton. & & Modian frea. & & Median conc. & & \\
\hline$S-102$ & all sizes & 12164 & $>$ & 2223 & $>$ & & $x$ & 2.B1E-05 & $\leq$ & \begin{tabular}{|r|}
14.7 \\
\end{tabular} & $>$ & \\
\hline & small & 4052 & $>$ & 219 & $>$ & $0=$ & $=$ & 3.70E-04 & $\underline{x}$ & 2.88 & $>$ & \\
\hline & medium & 4058 & 2 & 524 & $\leq$ & & $=$ & $3.99 \mathrm{E}-05$ & $\leq$ & 18.41 & $>$ & \\
\hline & large & 4056 & $>$ & 1480 & $\geq$ & $0=$ & $=$ & $1.71 \mathrm{E} \cdot 06$ & $\leq$ & 71.8 & $<$ & \\
\hline U-111 & all sizes & 11937 & $<$ & 738 & $\geq$ & & - & $3.39 E-05$ & $<$ & $5.7 x$ & $\geq$ & \\
\hline & small & 3977 & $\varepsilon$ & 124 & $\geq$ & & 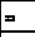 & $4.82 E \cdot 04$ & $<$ & $1.19=$ & $>$ & \\
\hline & medium & 3980 & $<$ & 316 & $\geq$ & & $=$ & 5.23E-05 & $\leq$ & 7.37 & $\geq$ & \\
\hline & large & 3980 & $<$ & 296. & $<$ & $0=$ & $\pi$ & $2.08 E \cdot 08$ & $<$ & $32.1>$ & $>$ & \\
\hline T-203 & all sizes & 12226 & $>$ & 1592 & $>$ & $116>$ & $>$ & $2.47 \mathrm{E} .06$ & $<$ & 8.95 & $\geq$ & \\
\hline & small & 4074 & $>$ & 4 & $\leq$ & $0=$ & $=$ & $3.30 \mathrm{E}-05$ & $\leq$ & 2.29 & $>$ & \\
\hline & medium & 4076 & $>$ & 208 & $\geq$ & $01=$ & $=$ & $4.00 E-06$ & $k$ & 13.4 & $\geq$ & \\
\hline & large & 4076 & $>$ & 1380 & 2 & 116:? & $\geq$ & $1.34 E-07$ & $<$ & $61.4 \mathrm{~S}$ & $>$ & \\
\hline$B-\$ 11$ & ail sizes & 11709 & $\leq$ & 220 & $<$ & ol= & II & 1.80E-05 & $\leq$ & 1.99 & $\geq$ & \\
\hline & small & 3901 & $<$ & 12 & $\geq$ & $0=$ & $=$ & $1.89 \mathrm{E}-04$ & $<$ & 0.526 & $\geq$ & \\
\hline & medium & 3904 & $<$ & 72 & $\geq$ & $0 \mid=$ & $=$ & $2.95 \mathrm{E}-05$ & $<$ & 3.28 & 2 & \\
\hline & large & 3904 & $<$ & 136 & $<$ & 0. & + & $1.02 E-06$ & $\leq$ & 13.4 & & \\
\hline & & & & & & & & & & & & \\
\hline & & & & & & & & & & & & \\
\hline & & & & & & & & & & & & \\
\hline & & & & & & & & & & & & \\
\hline & & & & & & & & & & & & \\
\hline & & & & & & & & & & & & \\
\hline & & & & & & & & & & & & \\
\hline & & & & & & & & & & & & \\
\hline & & & & & & & & & & & & \\
\hline & & & & & & & & & & & & \\
\hline & & & & & & & & & & & & \\
\hline & & & & & & & & & & & & \\
\hline & & & & & & & & & & & & \\
\hline & & & & i & & & & & & & & \\
\hline
\end{tabular}




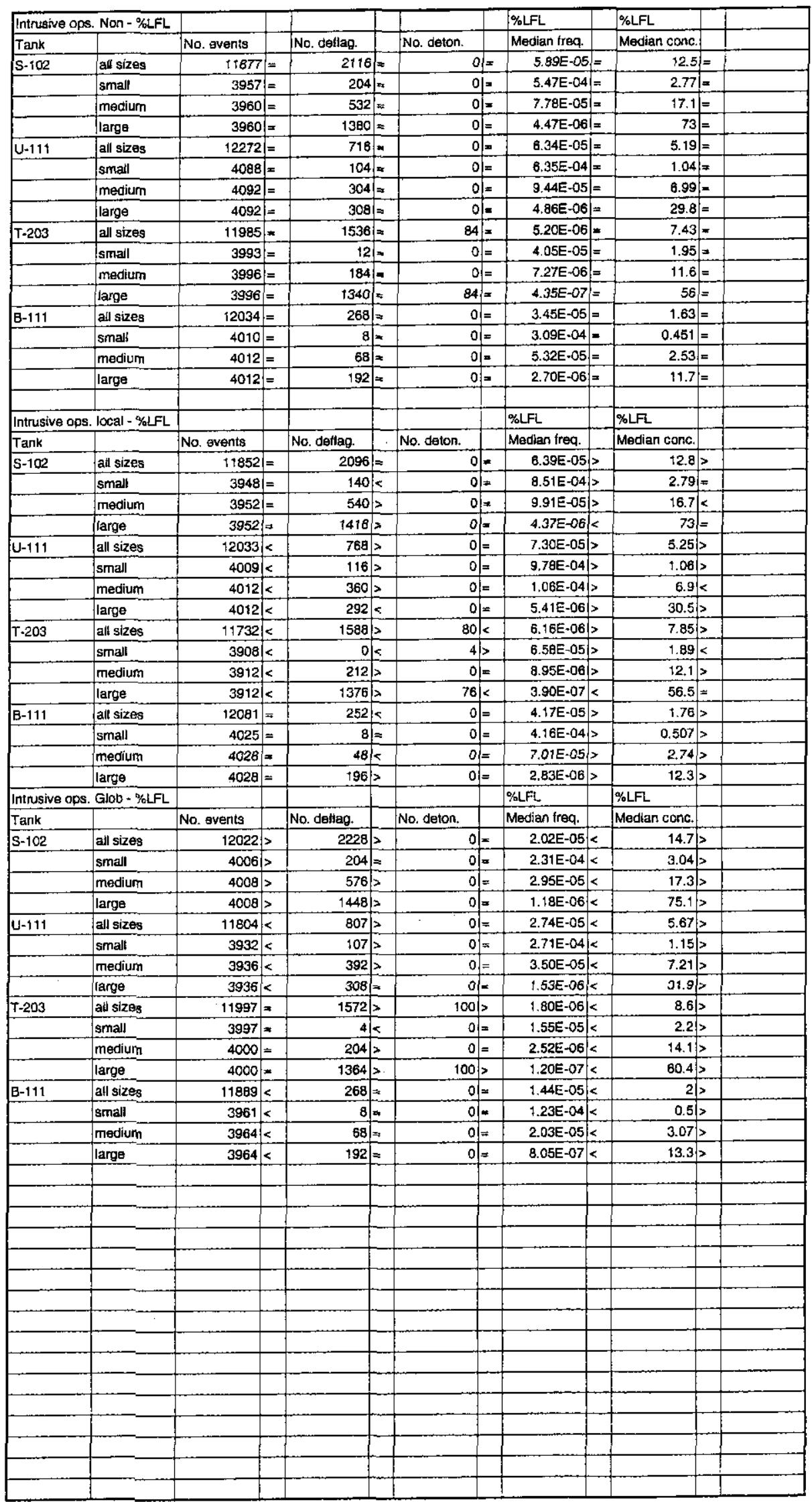




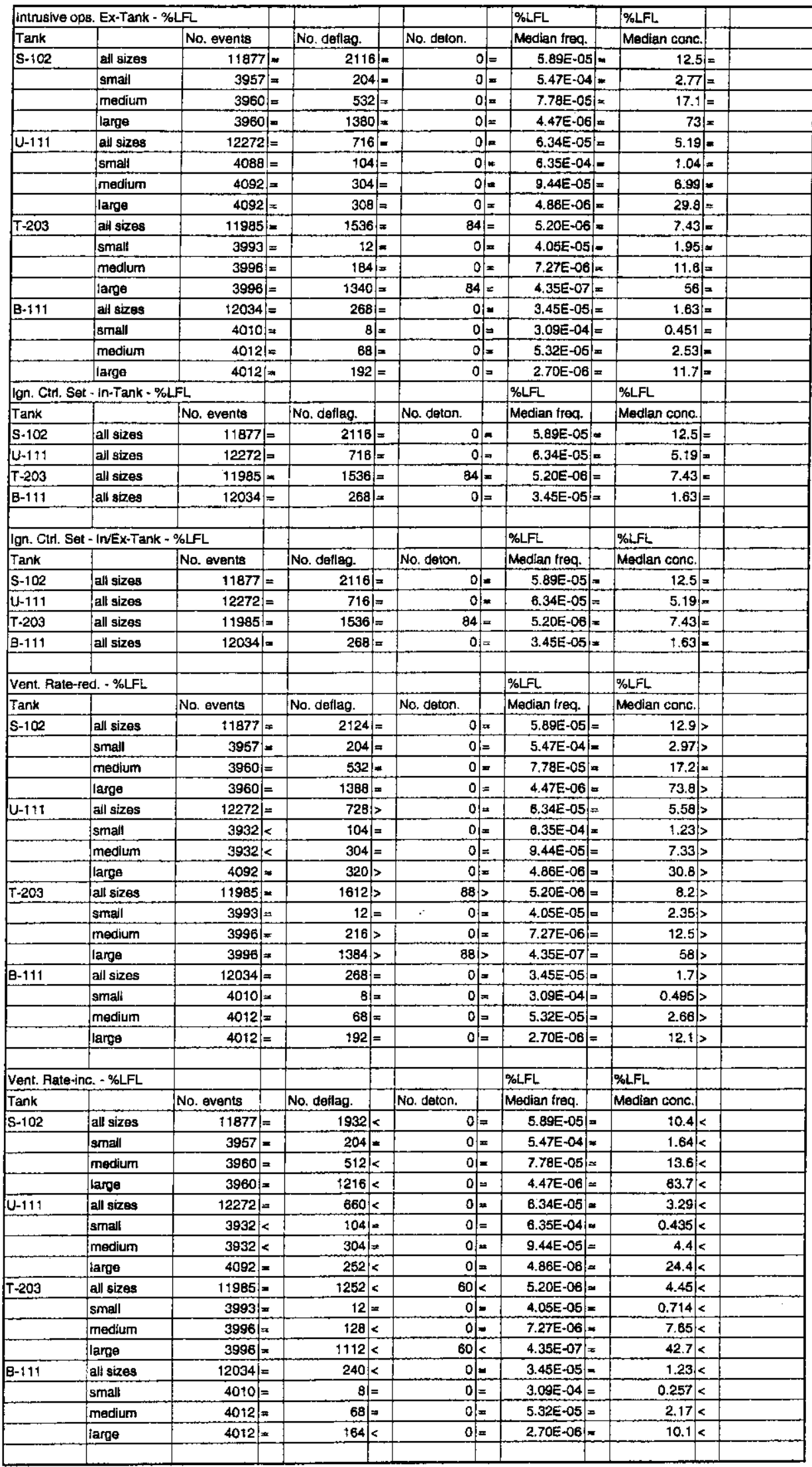




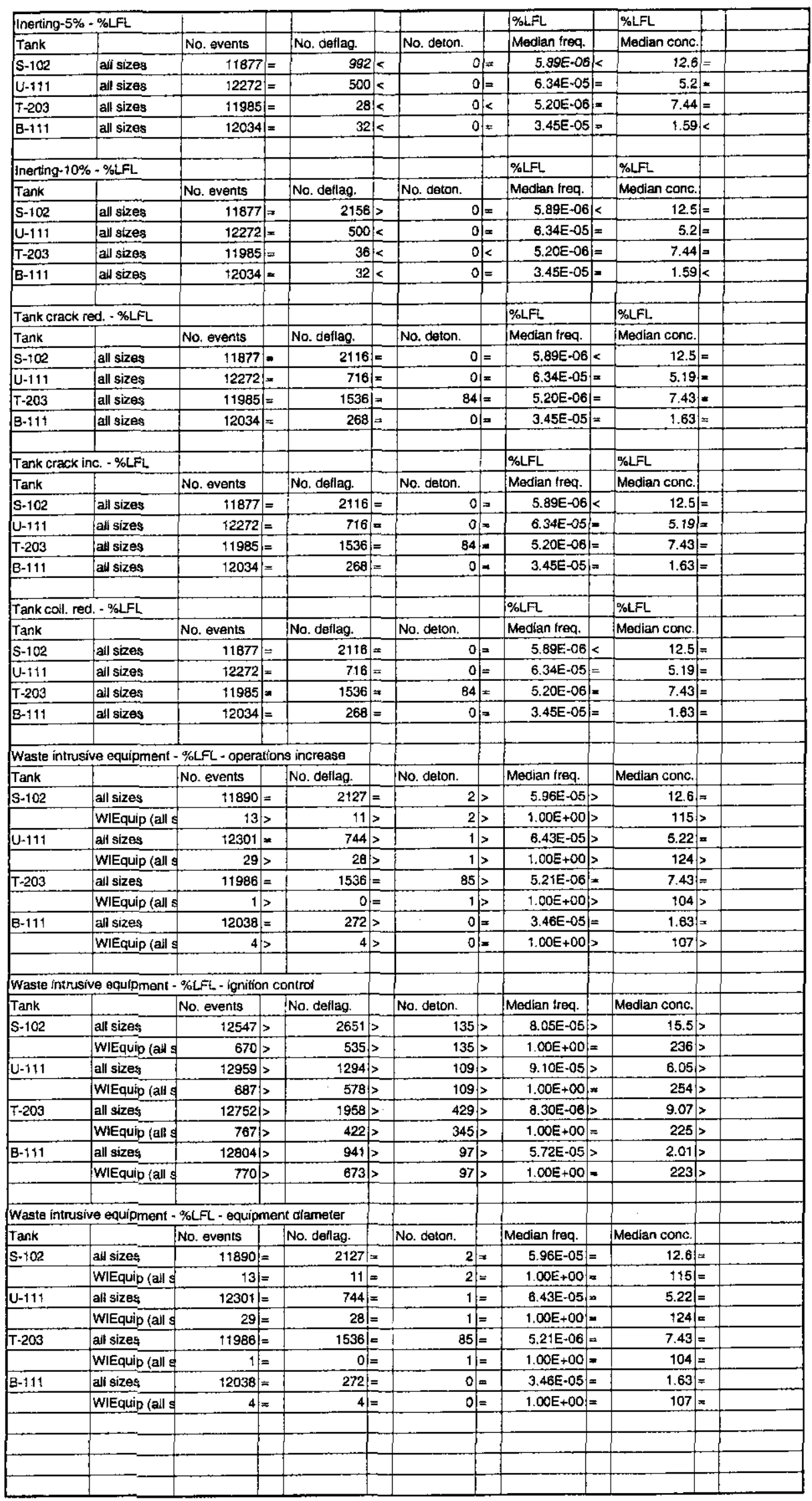




\begin{tabular}{|c|c|c|c|c|c|c|c|c|c|c|c|c|}
\hline \multicolumn{6}{|c|}{ Waste intrusive equipment - \%LFL - equipment length } & \multirow[b]{2}{*}{ No. deton. } & & \multirow[b]{2}{*}{ Median frea. } & & \multirow[b]{2}{*}{ Median conc. } & \multirow{2}{*}{\multicolumn{2}{|c|}{1}} \\
\hline Tank & & No. events & & No. deflag. & & & & & & & & \\
\hline \multirow[t]{2}{*}{$5-102$} & all sizes & 11890 & $\mathbf{z}$ & 2127 & $\bar{x}$ & 2 & $=$ & 5.96E-05 & $=$ & 12.6 & & \\
\hline & WIEquip (ail s & 13 & n & 11 & 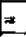 & 2 & $=$ & $1.00 E+00$ & $=$ & +15 & $=$ & \\
\hline \multirow[t]{2}{*}{$\underline{U}-1 \pm 1$} & all sizes & 12301 & $=$ & 744 & $=$ & & $=$ & $6.43 E-05$ & $\pi$ & $5.22=$ & $=$ & \\
\hline & WIEquip (alls & 29 & $=$ & 28 & $=$ & 1 & $=$ & $1.00 \mathrm{E}+00$ & 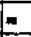 & $124=$ & $=$ & \\
\hline \multirow[t]{2}{*}{$T-203$} & ail sizes & 11986 & $=$ & 1536 & $=$ & 85 & $=$ & $5.21 \mathrm{E}-06$ & \pm & 7.43 & $=$ & \\
\hline & WIEquip <all s & 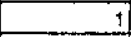 & $=$ & 야 & $=$ & & $*$ & $1.00 E+\infty$ & $=$ & $104=$ & $=$ & \\
\hline \multirow[t]{2}{*}{ B-111 } & all sizes & 12038 & $=$ & 272 & 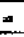 & 0 & $=$ & $3.46 E-05$ & $x$ & $1.63=$ & I & \\
\hline & WiEquip (ail s & 4 & $=$ & 4 & 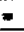 & 이 & $=$ & $1.00 E+00$ & $=$ & $107=$ & 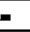 & \\
\hline & & & & & & & & & & & & \\
\hline & & & & & & & & & & & & \\
\hline & & & & & & & & & & & & \\
\hline & & & & & & & & & & & & \\
\hline & & & & & & & & & & & & \\
\hline & & & & & & & & & & & & \\
\hline & & & & & & & & & & & & \\
\hline & & & & & & & & & & & & \\
\hline & & & & & & & & . & & & & \\
\hline
\end{tabular}




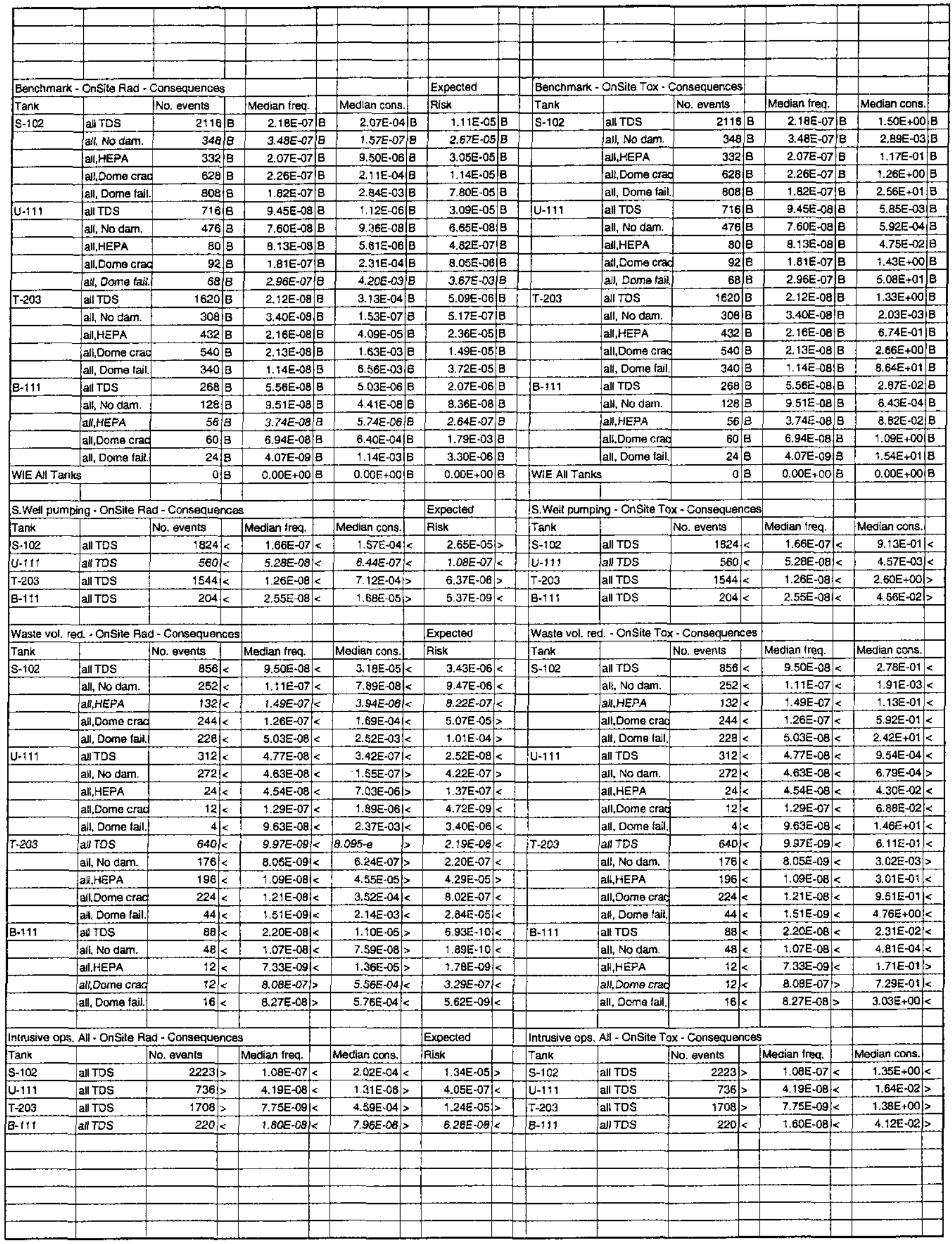




\begin{tabular}{|c|c|c|c|c|c|c|c|c|c|c|c|}
\hline \multicolumn{4}{|c|}{ Intrusive ops. Non - OnSite Rad - Consequences } & \multirow[b]{2}{*}{ Median cons. } & \multirow{2}{*}{\begin{tabular}{|l} 
Expected \\
Pisk
\end{tabular}} & \multicolumn{4}{|c|}{ Intrusive aps. Non - OnSite Tox - Consequences } & \multirow{2}{*}{\multicolumn{2}{|c|}{ Median cons. }} \\
\hline Tank & & No. events & Median Ireq. & & & Tank & & No. events & Median freq. & & \\
\hline S-102 & all TDS & $2116=$ & $2.19 \mathrm{E}-07$ & $2.07 \varepsilon-04$ & $1.15 \mathrm{E}-05$ & $S-102$ & all TDS & 2116 & \begin{tabular}{|r|}
$2.19 E-07$ \\
\end{tabular} & $1.50 \mathrm{E}+00$ & \\
\hline $\mathrm{U}-111$ & all TDS & $716=$ & $9.45 \mathrm{E}-08 \mathrm{x}=$ & $1.12 \mathrm{E}-06$ & $3.09 E-05=$ & U-111 & ail TDS & $716=$ & $9.45 E-08$ & $5.85 E-03$ & \\
\hline $\mathrm{T}-203$ & all TDS & $10201=$ & $2.18 E-08$ & $3.13 \mathrm{E}-04$ & $5.11 \mathrm{E}-06$ & $T-203$ & all TDS & 1620 & $2.18 E-08$ & $1.39 E+00$ & $\mid=$ \\
\hline B-111 & all TDS & $268=$ & $5.67 \mathrm{E}-08$ & $5.03 E-06=$ & 2.07E-06 $=$ & $B-111$ & all TDS & 268 & $5.67 \mathrm{E}-08$ & $2.87 E-02$ & \\
\hline \multicolumn{4}{|c|}{ Intrusive ops. locat - OnSilte Aad - Consequences } & & Expected & & \multicolumn{3}{|c|}{ Intrusive ops. local - OnSite Tox - Consequences } & & \\
\hline $\begin{array}{l}\text { Intrusive } \\
\text { Tank } \\
\end{array}$ & & No. events & Median freq. & Median cons. & $\begin{array}{l}\text { Expecited } \\
\text { Risk }\end{array}$ & Tank & & Na. events & Median freq. & Median cons. & \\
\hline S-102 & ail TDS & $2096=$ & $2.35 E-07$ & $1,63 E-04$ & $3.61 \mathrm{E}-05>$ & S-102 & aA TDS & $2096=$ & $2.35 E-07$ & $8.86 \mathrm{E}-01$ & T< \\
\hline $\mathrm{U}-111$ & all TOS & $768>$ & $1.27 E-07$ & $1.24 E-067$ & $8.99 \mathrm{E}-08 \mathrm{C}$ & $U=111$ & all TDS & 788 & $1.27 E-07$ & $7.73 E-03$ & $3>$ \\
\hline$T-203$ & all TOS & $1668>$ & $1.88 \mathrm{E}-08 \mathrm{~S}_{\mathrm{S}}$ & $4.28 E-04]$ & $5.97 \mathrm{E}-06$ & $T-203$ & all TDS & 1668 , & $1.88 \mathrm{E}-08$ & $1.91 E+\infty$ & $\mid>$ \\
\hline B-111 & all TOS & $252 \mid<$ & $7.16 E-08$ & $3.38 \mathrm{E}-06$ & $1.86 \mathrm{E}-07 \mathrm{~K}_{\mathrm{S}}$ & $\mathrm{B}-111$ & aal TDS & $252<$ & $7.16 \mathrm{E}-00$ & $3.54 \mathrm{E}-02$ & $>$ \\
\hline & & & & & & & & & & & \\
\hline \multicolumn{4}{|c|}{ Intrusive ops. Glob - OnSite Rad - Consequences } & & Expected & Intusive & s. Giob - 0 & te Tox-Conseque & ences & & \\
\hline Tank & & No. events & Median freq. & Median cons. & Risk & Tank & & No. events & Median freq. & Median cons. & \\
\hline S-102 & all TOS & $2228>$ & $9.05 E-08<$ & $1.74 E-04$ & $1.82 \mathrm{E}-04 \mathrm{~S}$ & s-102 & aH TDS & $22281>$ & $9.05 \mathrm{E}-08$ & $1.28 \mathrm{E}+00$ & \\
\hline U-111 & ail TDS & $807]>$ & $2.49 \mathrm{E}-0 \mathrm{0B}<$ & $1.28 \mathrm{E}-06$ & $4.23 \mathrm{E}-07 \mid<$ & $\mathrm{U}-111$ & all TDS & 807 & $2.49 E-08$ & $9.23 \mathrm{E}-03$ & \\
\hline$T-203$ & all TDS & $1672>$ & $6.28 \mathrm{E}-09 \mathrm{~S}$ & $|4.29 \mathrm{E}-04|>$ & $4.98 E-06<$ & $T-203$ & ail TDS & $1672>$ & $6.28 E-09$ & $1.47 E+00$ & \\
\hline B-111 & at1 TOS & $268=$ & $1.49 \mathrm{E}-08 \mathrm{C}$ & $4.65 E-06<$ & $4.64 \mathrm{E}-08 \mathrm{C}$ & $B-111$ & all TDS & $268=$ & 1.49E-08 & $9.22 \mathrm{E}-02$ & \\
\hline & & & & & & & & & & & \\
\hline Intrusive & Ex-Tank & nSite Rad - Conse & quences & & Expected & intrusive & 8. Ex-Tank & Insite Tox - Conse & aquences & & \\
\hline Tank & & \begin{tabular}{|l|} 
No. events \\
\end{tabular} & Median freq. & Median cons. & Risk & Tank & & No. events & Median freq. & Median cons. & \\
\hline S-102 & at TDS & $2116=$ & $|2.41 \mathrm{E}-07|>$ & $|2.07 E-04|=$ & $\mid 1.15 \mathrm{E}-05>$ & $S-102$ & all TDS & $2116=$ & $2.41 \mathrm{E}-07$ & $1.51 \mathrm{E}+00$ & $=$ \\
\hline $\mathrm{U}-111$ & ail TDS & $716=$ & $1.01 \mathrm{E}-07>$ & $1.12 E-06=$ & $3.09 E-05 \mid=$ & U-111 & all TDS & $716=$ & $1.01 E-07$ & $5.85 E-03$ & $=$ \\
\hline$T-203$ & all TOS & $1620=$ & $2.63 E-08>$ & $3.13 \mathrm{E}-04$ & $5.28 E-06>$ & $T-203$ & all TOS & $1620=$ & $2.63 E-09$ & $1.38 E+00$ & \\
\hline $\mathrm{B}-111$ & all TOS & $268 \mid=$ & $5.67 \mathrm{E}-08 \mid>$ & $5.03 E-0 B=$ & $2.07 E-06=$ & $B \cdot 111$ & all TDS & $268 \mid=$ & $5.67 \mathrm{E}-08$ & $2.87 \mathrm{E}-02$ & \\
\hline & & & & & & & & & & & \\
\hline Ign. Ctri. & - In-Tank & Site Rad - Consec & quences & & Expected & Ign. Ctrl. & t-In-Tank & ISite Tox - Conser & quences & & \\
\hline Tank & & \begin{tabular}{|l|} 
No. events \\
\end{tabular} & |Medlan freq. & Median cons. & Aisk & Tank & & \begin{tabular}{|l|} 
No. events \\
\end{tabular} & Meclan frea. & Modian cons. & \\
\hline S-102 & all TDS & $2116=$ & $\mid 2.86 E-07>$ & $2.07 \mathrm{E}-04=$ & $|1.37 \mathrm{E}-05\rangle$ & S-102 & all TDS & 2116 & $\mid 2.86 \mathrm{E}-07$ & $1.55 E+00$ & \\
\hline $\mathrm{U}-111$ & all TDS & $716=$ & $|1.61 \mathrm{E}-07|>$ & $1,12 \mathrm{E}-0 \mathrm{~B}=$ & $3.52 E-05>$ & Tu-111 & tall TDS & $71 B=$ & $\mid 1.6 \mathrm{TE}-07$ & $7.40 E-03$ & \\
\hline$T .203$ & all TDS & $1620=$ & $2.46 \mathrm{E}-08$ & $3.13 E-04=$ & $6.21 \mathrm{E}-06>$ & $T-203$ & all TOS & $1620=$ & $2.46 E-08=$ & $1,36 \mathrm{E}+00$ & \\
\hline B-111 & ail TDS & $268=$ & B.99E-08> & $5.03 E-06=$ & $2.66 \mathrm{E}-06 \mid>$ & B-111 & ail TDS & $268=$ & $6.99 E-08$ & 3.36E-02 & \\
\hline & & & & & & & & & & & \\
\hline & & & & & & & & & & & \\
\hline & & & & & & & & & & & \\
\hline Ign. Cir. & - InVEx-Tar & OnSite Rad - Con & sequences & & Expacted & Ign. Cth. & $t-\operatorname{In} / E x-T a$ & Onsite Tox - Cor & isequences & & \\
\hline Tank & & No. events & Median freq. & IMedian cons. & Fisk & Tank & & No. events & Median freg. & Medlan cons. & \\
\hline S-102 & ail TDS & $2116=$ & $2.48 E-07>$ & $2.07 \mathrm{E}-04=$ & 1.10E-05 = & $s-102$ & all TOS & $2116=$ & $\mid 1.82 \mathrm{E}-07$ & $1.50 \mathrm{E}+00$ & \\
\hline$U-111$ & all TOS & $716 \times$ & $1.61 \mathrm{E}-07>$ & . $12 \mathrm{E}-06=$ & $3.52 E-05>$ & $U-111$ & all TOS & $716=$ & $1.61 \mathrm{E}-07>$ & $7.40 E-03$ & \\
\hline$T-203$ & all TOS & $1620=$ & $2.15 E-08>$ & $3.13 \mathrm{E}-04=$ & $6.17 E-06 \mid>$ & $T-203$ & all TDS & $1620=$ & $2.15 E-08>$ & $1.38 \mathrm{E}+00=$ & \\
\hline B-111 & all TOS & $268=$ & $5.43 \mathrm{E}-0 \mathrm{OB}<\mathrm{C}$ & $5.03 E-06=$ & 2.07E.06 $=$ & $\mathrm{B}+111$ & all TOS & $268=$ & $5.43 E-08$ & $2.87 E-02]=$ & \\
\hline & & & & $\therefore \quad-1$ & & & & & & & \\
\hline Vent. Aa & od. - Onsite & ad - Consequence & & & Expacted & Vent. Ra & ed. - Onsit & ox - Consequence & & & \\
\hline Tank & & No. events & Median freq. & Median cons. & Fisk & Tank & & No. events & Median freq. & Median cons. & \\
\hline S-102 & at TDS & $|2114|=$ & $3.05 \mathrm{E}-07>$ & $2.07 \mathrm{E}-04=$ & $|1.15 E-05\rangle$ & S-102 & al TOS & $2114=$ & $3.05 E-07>$ & $1.51 \mathrm{E}+00$ & \\
\hline U-11t & all TOS & $728>$ & $1,15 \mathrm{E}-07>$ & $1.15 \mathrm{E}-06>$ & $3.09 E-05 \mid=$ & U.11t & all TDS & $728 ?$ & $1.15 E \cdot 07>$ & 8.16E-03, & \\
\hline$T-203$ & all TDS & $1700>$ & $3.57 \mathrm{E}-0.01>$ & $3.10 \mathrm{E}-04$ & $5.79 E-06 \mid>$ & $T-203$ & all TDS & $1700>>$ & $3.57 E-08>$ & $1.42 E+\infty$ & \\
\hline B-111 & all TDS & $268=$ & $7.13 E-08>$ & $5.03 E-06=$ & $2.07 \mathrm{E}-08 \mid \mathrm{a}$ & $B-111$ & ail TDS & $268=$ & $7.13 \mathrm{E}-0 \mathrm{\theta}>$ & $3.36 E-02$ & \\
\hline & & & & & & & & & & & \\
\hline Vent. Aa & c. - OnSile & d - Consequences & & & Expected & Vent. Rat & nc. - OnSite & $x$ - Consequence & & & \\
\hline Tank & & No. events & Median freq. & Medlan cons. & Fisk & Tank & & No. events & Modian freq. & Modian cons. & \\
\hline S-102 & all TDS & $1932<$ & \begin{tabular}{|r|r|} 
& $1.35 E-07<$ \\
\end{tabular} & $2.32 E-04>$ & $\mid 1.10 E-05=$ & $\mathrm{S}-102$ & all TDS & $\mid 1932<$ & $1.35 \mathrm{E}-07 \mathrm{~K}$ & $1.50 \mathrm{E}+00$ & \\
\hline$U-111$ & all TDS & $660<$ & $7.53 \mathrm{E}-0 \mathrm{OB}<$ & $7.93 \mathrm{E}-07<$ & $3.09 \mathrm{E}-05=$ & $U-111$ & a!l TDS & $660<$ & $7.53 E-08<$ & $3.99 E-03$ & \\
\hline T-203 & all TDS & $1312<$ & $1.34 \mathrm{E}-08 \mathrm{~K}<$ & $2.51 E-04<$ & $2.87 E-06<<$ & $T-203$ & all TDS & $1312 k$ & $1.34 \mathrm{E}-08<$ & $1.22 E+\infty 0$ & \\
\hline B-111 & ail TDS & $240<\leq$ & 5.09E-08|< & $3.53 E-06<$ & $2.06 \mathrm{E}-06=$ & $B-111$ & all TDS & $240 \mathrm{~K}$ & $5.09 \mathrm{E}-08 \mathrm{C}$ & $2.20 E-02$ & \\
\hline & & & & & & & & & & & \\
\hline Inerting-5 & Onsite $\mathrm{Fla}$ & Consequences & & & Expectad & Inerting. & - OnSite To & Consequencer & & & \\
\hline Tank & & \begin{tabular}{|l|} 
No. events \\
\end{tabular} & Median freq. & Modian cons. & \begin{tabular}{|l|l|l|} 
Risk \\
\end{tabular} & Tank & & No. avents & Median ireq. & Median cons. & \\
\hline S-102 & all TDS & \begin{tabular}{rl|r|}
$992<$ \\
\end{tabular} & $9.35 E-08<$ & $|7.39 E-05|_{<}$ & $3.09 E-06<$ & S-102 & all TOS & 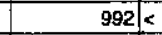 & $\mid 9.35 \mathrm{E}-0 \mathrm{~B}$ & $4.14 \mathrm{E}-01$ & \\
\hline$U-111$ & all TOS & $500<$ & $7.57 \mathrm{E}-08<<$ & $3.13 E-07<<$ & $2.15 E-05<<$ & $\mathrm{U}-111$ & ail TOS & $500 \mid<$ & $7.57 E-08<$ & $1.16 E-03$ & \\
\hline$T-203$ & all TDS & $28 /<$ & $3.01 E-08>$ & $3.92 E-03>$ & $1.17 \mathrm{E}-08<$ & $T-203$ & all TOS & $28<$ & $3.01 E-0.08$ & $2.10 E+01>$ & \\
\hline E.111 & all TDS & $32<$ & $3.40 \mathrm{E}-08 \mathrm{~K}$ & $\begin{array}{l}1.00 E-08< \\
\end{array}$ & $7.48 E-11<$ & $B-111$ & all TDS & $32 \mathrm{~K}$ & $3.40 E-0 B<$ & $2.86 \mathrm{E}-03<$ & \\
\hline & & & & & & & & & & & \\
\hline Inerting- & - Onsiter & Consequences & & & Expected & Inerting-1 & OnSite 7 & - Consequences & & & \\
\hline Tank & & No. events & Medlan freq. & Median cons. & \begin{tabular}{|l|l|} 
Risk \\
\end{tabular} & Tank & & No. events & Median freq. & Medlan cons. & \\
\hline S-102 & all TOS & $2156>$ & $|2.25 E-07|>$ & $1.84 \mathrm{E}-04 / \mathrm{K}$ & $1.27 \mathrm{E}-05>$ & S. 102 & all TDS & $2156>$ & $\mid 2.25 \mathrm{E}-07>$ & $9.01 E-01$ & \\
\hline$U-\$ 11$ & all TOS & $500<$ & $7.57 E-08<\leq$ & \begin{tabular}{|l|l|l|}
$3.13 E-07 /<$ \\
\end{tabular} & $2.15 E-05<$ & $U-111$ & all TDS & $500<$ & $7.57 \mathrm{E}-08<$ & $1.18 \mathrm{E}-03$ & \\
\hline$T-203$ & ant TDS & $36<$ & $8.82 E-09<<$ & $5.30 \mathrm{E}-03 \mid>$ & $1.69 \mathrm{E}-08 \mid<$ & $T-203$ & all TOS & $38<$ & $8,82 \mathrm{E}-09<$ & $3.41 \mathrm{E}+01>$ & \\
\hline-111 & all TDS & $32<$ & $3.40 E-08<<$ & $1.00 \mathrm{E}-08 \mathrm{i}_{\mathrm{K}}$ & $7.48 E-11 /<$ & $B-111$ & all TOS & $32<$ & $3.40 E-08<$ & $2.86 \mathrm{E}-03<$ & \\
\hline
\end{tabular}




\begin{tabular}{|c|c|c|c|c|c|c|c|c|c|c|c|}
\hline \multicolumn{4}{|c|}{ Tank crack red. - OnSite Rad - Consequences } & \multirow[b]{2}{*}{ Median cons. } & \multirow{2}{*}{\begin{tabular}{|l|} 
Expected \\
Risk
\end{tabular}} & \multicolumn{3}{|c|}{ Tank crack red. - OnSite Tox - Consequences } & \multirow{2}{*}{ Mecilan freq. } & \multirow[b]{2}{*}{ Median cons. } & \\
\hline Tank & & No. events & Median freq. & & & Tank & & No. events & & & \\
\hline \multirow[t]{5}{*}{$\mathrm{s}-102$} & all TDS & $2116=$ & $2.1 \mathrm{AE}-07=$ & $2.14 E-04>$ & $9.97 \mathrm{E}-06<$ & s. 102 & all TOS & $2116=$ & $2.18 \mathrm{E}-07$ & $1.70 E+00=$ & \\
\hline & all, No dam. & $348=$ & $1.80 \mathrm{E}+02>$ & $1.57 E-07=$ & $2.67 \mathrm{E}-055=$ & & all, No dam. & 348 & $3.48 E-07$ & $2.89 E-03$ & \\
\hline & all,HEPA & $180 / 5$ & $7,80 E+02>$ & $1.03 E-06<$ & $1.56 \mathrm{E}-06 \mathrm{~K}$ & & all,YHEPA & $180<5$ & $2.01 E-07$ & $6.11 E-02$ & \\
\hline & all, Dome craed & $780 \mid \geq$ & $8.08 \mathrm{E}+02>$ & $2.03 E-04<<$ & $1.45 E-06 / 5$ & & ail,Dome crad & 780 ? & $2.26 E-07$ & $1.12 \mathrm{E}+00 \mathrm{a}$ & \\
\hline & all, Dome fail. & 808 & $1.82 E-07=$ & $2.84 \mathrm{E}-03=$ & $7.80 E-05=$ & & ah, Dome tail: & $808=$ & $1.82 \mathrm{E}-07$ & $2.56 \mathrm{E}+01=$ & \\
\hline \multirow[t]{5}{*}{$U-111$} & all TDS & $716=$ & $9.45 E-08:=$ & $1.17 \mathrm{E}-06$ & $3.09 E-05=$ & $U-111$ & ant TDS & $716=$ & $9.45 E-08=$ & $9.14 \mathrm{E}-03$ & \\
\hline & all, No dam. & $476=$ & $7.60 \mathrm{E}-0 \mathrm{8}=$ & $9.36 \mathrm{E}-08=$ & $6.65 E-08=$ & & all, No dam. & 476 & $7,60 \mathrm{E}-08$ & $5.92 \mathrm{E}-04$ & \\
\hline & aAl.MEPA & $56<$ & $6.53 \mathrm{E}-08 / \mathrm{K}$ & $1.09 E-05>$ & $8.74 \mathrm{E}-07>$ & & \begin{tabular}{|l|l|l} 
All,HEPA \\
\end{tabular} & $58<<$ & $6.59 \mathrm{E}-08$ & $4.75 \mathrm{E}-02=$ & \\
\hline & all.Dome crac & $116>$ & 1.81E-07 $=$ & $1.36 E-04<$ & $7.23 \mathrm{E}-06<<$ & & all,Dome crac & $116>$ & $1.81 E-07$ & $9.86 E-01$ & \\
\hline & all, Dome fail. & $68=$ & $2.96 \mathrm{E}-07=$ & $4.20 E-03=$ & $3.67 \mathrm{E}-03=$ & & all, Dome fail. & 68 & $2.96 \mathrm{E}-07$ & $5.08 \mathrm{E}+01=$ & \\
\hline$T-203$ & all TOS & $1620=$ & $2.12 E \cdot 08=$ & $5.81 E-04>$ & $5.24 \mathrm{E}-06\rangle$ & $T-203$ & \begin{tabular}{|l|l|l|} 
TDS \\
\end{tabular} & $1620=$ & $2.12 E-08=$ & $2.04 E+000$ & \\
\hline & all, No dam. & $308=$ & $3.40 \mathrm{E}-0 \mathrm{OB}=$ & $1.53 E-07=$ & $5.17 \mathrm{E}-07=$ & & all, No dam. & $308=$ & $3.40 \mathrm{E}-08$ & $2.30 E-03,7$ & \\
\hline & all,HEPA & $252<$ & $3.72 E-08 \mid>$ & $5.40 E-05>$ & $2.09 E-05 /<$ & & all,HEPA & $252 k$ & $3.72 E-08$ & $9.12 E-01]=$ & \\
\hline & all,Dome crac & $720>$ & $1.95 \mathrm{E}-08 \mathrm{~S}$ & $|1.70 \mathrm{E}-03|>$ & $|1.42 E-05| \mathrm{K}$ & & all,Dome crac & $7201>$ & $1.95 \mathrm{E}-08$ & $3.73 E+00>$ & \\
\hline & all, Dome fail. & $340=$ & $1.14 \mathrm{E}-08=$ & $6.56 \mathrm{E}-03=$ & $3.72 E-05=$ & & all, Dome fail. & $340=$ & $1.14 E-08=$ & $8.64 E+01=$ & \\
\hline B-111 & aal TDS & $268=$ & $5.56 \mathrm{E}-08=$ & $5.03 \mathrm{E}-06=$ & $2.00 E-06=$ & B-111 & all TDS & 268. & $5.56 \mathrm{E}-08=$ & $2.296-02$ & \\
\hline & all, No darn. & $126=$ & $9.51 \mathrm{E}-0 \mathrm{0} \times$ & $4.41 \mathrm{E}-08=$ & $8.36 E-08 \mathrm{x}$ & & all, No dam. & $128=$ & $9.51 E-08=$ & $6.43 E-04=$ & \\
\hline & all:HEPA & $24<$ & $3.74 E-08=$ & $5.53 E-06<$ & 3.11E-07>> & & all,HEPA & $24<$ & $3.74 E-08=$ & $3.48 E-02$ & \\
\hline & ali,Dome crag & $92>$ & $5.56 \mathrm{E}-\left.08\right|_{<}$ & $1.99 E-04<$ & $1,78 \mathrm{E}-03=$ & & all,Dome crad & $92>$ & $5.56 \mathrm{E}-08$. & 4.72E-01 & \\
\hline & all, Dome fail. & $24=$ & $4.07 \mathrm{E}-09=$ & $1.14 \mathrm{E}-03=$ & $3.30 \mathrm{E}-06=$ & & all, Dome tail. & $24=$ & $4.07 E-09=$ & $1.54 E+01$ & \\
\hline & & & & & & & & & & & \\
\hline Tank cr & c. - OnSite $\mathrm{Rad}$ & d - Consequences & & & Expected & Tank cr & c. - OnSite Tox & $x$ - Consequence & & & \\
\hline Tank & & No events & Median freq. & Median cons. & Risk & Tank & & No. events & Median freq. & Median cons. & \\
\hline S-102 & all TOS & $2116=$ & $2.18 \mathrm{E}-07=$ & $|1.92 E-04|<$ & $|1.05 E-05| \mathrm{K}$ & s-102 & all TDS & $2116=$ & $2.18 \mathrm{E}-07$ & $1.38 \mathrm{E}+00$ & \\
\hline & all, No dam. & $348=$ & $3.48 E-07=$ & $1.57 E-07=$ & $2.87 E-05=$ & & all, No dam. & $348=$ & $3.48 \mathrm{E}-07=$ & $2.89 \mathrm{E}-03=$ & \\
\hline & \begin{tabular}{|l|} 
ail,HEPA \\
\end{tabular} & $520>$ & $2.28 E-07>$ & $1.11 E-05>$ & $2.23 \mathrm{E}-05<<$ & & all, HEPA & $520>$ & $2.28 \mathrm{EE}-07$ & $6.11 \mathrm{E}-02$ & \\
\hline & all,Dome crad & $440<5$ & $\begin{array}{l}1.96 \mathrm{E}-07<< \\
\end{array}$ & $\mid 1.93 \mathrm{E}-04<$ & 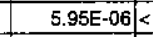 & & all,Dome crac & $440<$ & $1.96 \mathrm{E}-07$ & $1.12 E+\infty$ & \\
\hline & all, Dome fail. & $808=$ & $1.82 E-07=$ & $2.84 \mathrm{E}-03 \mathrm{j} \mid \mathrm{F}$ & $7.80 \mathrm{E}-05=$ & & all, Dome fail. & $800=$ & $1.82 \mathrm{E}-07=$ & $2.56 \mathrm{E}+01$ & \\
\hline $\mathrm{U}-111$ & at TDS & $\left.716\right|_{x}$ & $9.45 \mathrm{E}-08=$ & 9.27E-07< & 3.09E-05 $=$ & $U-111$ & ail TDS & $716=$ & $9.45 E-08=$ & $4.94 E-03$ & \\
\hline & ail, No dam. & $476=$ & $7.60 \mathrm{E}-00=$ & $9.36 \mathrm{E}-08=$ & $6.65 E-\left.08\right|_{-}$ & & all, No dam. & $476=$ & $7.60 \mathrm{E}-08=$ & $5.92 \mathrm{E}-04=$ & \\
\hline & anl,HEPA & $112>$ & $1.00 \mathrm{E}-07>$ & $5.61 \mathrm{E}-06=$ & $3.06 E-07<<$ & & all,HEPA & $112>$ & $1.00 \mathrm{E}-07$ & $5.31 \mathrm{E}-02=$ & \\
\hline & all,Dome crac & $60<$ & $1,81 \mathrm{E}-07=$ & $3.78 \mathrm{E} \cdot 05<\mathrm{K}$ & $5.61 E-08<$ & & all,Dome crac & $60 k$ & $1.81 \mathrm{E} \cdot 07=$ & $5.12 \mathrm{E}-01$ & \\
\hline & all, Dorne fail. & $68=$ & $2.96 \mathrm{E} \cdot 07=$ & $4.20 \mathrm{E}-03=$ & $3.67 E-03=$ & & all, Dome fail. & $68=$ & $2.96 E-07=$ & $5.08 E+01=$ & \\
\hline$T-203$ & ail TDS & $1620=$ & $2.12 E-08=$ & $1.07 E-04<$ & $4.55 E-06 \mid<$ & $T-203$ & all TDS & $1620=$ & $2.12 \mathrm{E}-08=$ & $9.34 E-01$ & \\
\hline & all, No dam. & $308=$ & $3.40 \mathrm{E}-00=$ & $\mid 1.53 \mathrm{E}-07=$ & $5.17 E-07 \mid=$ & & all. No dam. & $308=$ & $3.40 \mathrm{E}-0 \mathrm{OB}=$ & $2.30 \mathrm{E} \cdot 03$ & \\
\hline & all, HEPA & $772>$ & $2.45 E-08>$ & $4.83 \mathrm{E}-05>$ & $1.86 E-\left.05\right|_{\mathrm{K}}$ & & All.MEPA & $772>$ & $2.45 \mathrm{E}-08$ & $6.57 E-01$ & \\
\hline & all,Dome crac & $200<$ & $1.46 \mathrm{E}-08<$ & $1.77 \mathrm{E}-03 \mid>$ & $6.89 E-06<$ & & ali, Dome crac & $200 / \mathrm{K}$ & $\therefore .46 \mathrm{E}-08$ & $3.70 E+\infty$ & \\
\hline & atl, Dome fail. & $340=$ & $1.14 \mathrm{E}-08=$ & $6.56 \mathrm{E}-03=$ & $3.72 \mathrm{E}-05=$ & & all, Dome fail. & $340=$ & $1.14 \mathrm{E}-08=$ & $8.64 \varepsilon+01=$ & \\
\hline B.111 & ant TDS & $268=$ & $5.56 \mathrm{E}-08=$ & $3.57 \mathrm{E}-06<$ & $2.07 E-06$ & e-111 & all TDS & $268=$ & $5.56 \mathrm{E}-08=$ & $2.02 E-02$ & \\
\hline & ail, No dam. & $128=$ & $9.51 \mathrm{E}-08=$ & $4.41 \mathrm{E}-08=$ & 8. $36 E-08=$ & & all, No dam. & $128=$ & $9.51 \mathrm{E}-08=$ & $6.43 \mathrm{E}-04=$ & \\
\hline & ail,HEPA & 765 & $3.94 \mathrm{E}-09 \mid>$ & $6.04 \mathrm{E}-06>$ & $2.04 \mathrm{E}-07 \mathrm{~K}$ & & all,HEPA & $76 \mid$ & $3.94 E-08$ & $3.48 E-02$ & \\
\hline & all, Dome crac & $40 \mathrm{k}$ & $8.14 \mathrm{E}-0 \theta>$ & $-9.20 \mathrm{E} \cdot 04>$ & $1.80 E-03=$ & & all,Dome crad & $40 \mathrm{k}$ & $8.14 \mathrm{E}-08$ & $1.78 \mathrm{E}+00$ & \\
\hline & all, Dome tail. & $24=$ & $4.07 E-09=$ & $1.14 E-03=$ & $3.30 \mathrm{E}-06=$ & & all, Dome tail. & $24=$ & 4.07E-09:= & $1.54 \varepsilon+01=$ & \\
\hline & & & & & & & & & & & \\
\hline Tank co & 1. Onsite frad- & 1- Consequences & & & Expected & Tank co & 1. OnSile Tox - & - Consequences & & & \\
\hline Tank & & No. events & Median ireq. & Median cons. & \begin{tabular}{|l|l|l|l|l|} 
Risk & \\
\end{tabular} & Tank & & \begin{tabular}{|l|l|} 
No. events \\
\end{tabular} & Median freq & Median cons. & \\
\hline S-102 & all TDS & $2116=$ & $2.18 \mathrm{E}-07 \mid=$ & $5.41 \mathrm{E}-04>$ & $9.67 E-05>$ & S-102 & all TDS & $2116=$ & 2. $38 E-07$ & $5.24 E+00$ & \\
\hline & all, No dam. & $348=$ & $3.48 E-07 \mid=$ & 1.57E-07 $=$ & 2.67E-05 & & all, Na dam. & $348=$ & 3.4BE-07 & $2.89 \mathrm{E}-03=$ & \\
\hline & all,HEPA & $520>>$ & $2.07 \mathrm{E}-07 \mid=$ & $9.50 \mathrm{E}-06=$ & $3.05 E-05=$ & & all.HEPA & $520 \mid>$ & $2.07 E-07=$ & $1.17 E-01=$ & \\
\hline & all,Dome crad & $332<<$ & $2.99 \mathrm{E}-07>$ & $2.27 \mathrm{E}-04>$ & $2.52 E-05>$ & & all. Dome crac & $332 \leq \leq$ & $2.99 E-07$ & $1.36 \mathrm{E}+00$ & \\
\hline & all, Dome fail. & $976<$ & $1.925-07>$ & $3.49 E-03>$ & $8.46 \mathrm{E}-04>$ & & all, Dome fail. & $176<<$ & $1.92 \mathrm{E}-07$ & $3.38 E+01$ & \\
\hline $\mathrm{U}-111$ & all TDS & $716 \mid=$ & $9.45 E-08=$ & $1.31 E-06>$ & $3.11 \mathrm{E}-05=$ & U-111 & all TOS & $716=$ & $9.45 \mathrm{E}-08=$ & $5.85 E-03$ & \\
\hline & ail, No dam. & $476=$ & $7.60 E-08=$ & $9.36 \mathrm{E}-08=$ & $6.65 \mathrm{E}-0 \mathrm{~B}=$ & & all, No dam. & $476=$ & $7.60 \mathrm{E}-08$ & $5.92 E-04$ & \\
\hline & All, HEPA & $80=$ & $8.13 \mathrm{E}-00=$ & $5.61 \mathrm{E}-06$ & $4.82 \mathrm{E}-07=$ & & all,HEPA & $80=$ & $8.13 \mathrm{E}-08$ & $4.75 E-02$ & \\
\hline & all,Dome crac & $20<$ & $1.01 \mathrm{E}-07<$ & $8.18 E-04 \mid>$ & $3.46 \mathrm{E}-06 \mathrm{~s}$ & & all,Dome crac & $20<$ & $1.01 \mathrm{E}-07$ & $2.86 \mathrm{E}+00$ & \\
\hline & ail, Dome fail. & $140>$ & $2.32 \mathrm{E}-07<$ & $4.00 \mathrm{E}-03 \mathrm{~K}$ & $1.53 \mathrm{E}-03<$ & & all, Dome fail. & $140>$ & $2.32 \mathrm{E}-07$ & $2.94 E+01$ & \\
\hline $\mathrm{T}-203$ & all TDS & $1620=$ & $2.12 \mathrm{E}-0 \mathrm{a}=$ & $1.03 E-03>$ & $1.69 \mathrm{E}-05>$ & $T-203$ & all TDS & $1620=$ & $2.12 E-08=$ & $4.35 E+00$ & \\
\hline & all, No dam. & $308=$ & $3.40 E-08=$ & $1.53 \mathrm{E}-07=$ & $5.17 E-07=$ & & all, No dam. & $308:=$ & $3.40 E-08=$ & $2.30 \mathrm{E}-03$ & \\
\hline & all,HEPA & $432=$ & $2.45 \mathrm{E}-00 \mid>$ & $4.09 \mathrm{E}-05=$ & $2.36 \mathrm{E}-\left.05\right|_{=}$ & & all,HEPA & $432=$ & $2.45 E-08$ & $6.74 \mathrm{E}-01$ & \\
\hline & all,Dome crac & $88<$ & $1.46 \mathrm{E}-08$ & $1.18 E-03<$ & $5.36 \mathrm{E}-06 \mid<$ & & all,Dome crac & $88<5$ & $1.46 E-08$ & $1.90 \mathrm{E}+00$ & \\
\hline & all. Domra faii. & $792>$ & $1.14 \mathrm{E}-08=$ & $1.95 \mathrm{E}-02>$ & 9.87E-05i> & & all, Dome fail. & $792>$ & $1,14 E-08$ & $9.98 \mathrm{E}+01$ & \\
\hline $8-111$ & all TOS & $268 i=$ & $5.56 \mathrm{E}-08 \mid=$ & $5.03 \mathrm{E}-06=$ & $9.26 \mathrm{E}-07 / \mathrm{K}$ & B-111 & all TDS & $268=$ & $5.56 \mathrm{E}-08=$ & $2.87 \mathrm{E}-02$ & \\
\hline & all, No dam. & $128=$ & $9.51 \mathrm{E}-08=$ & $4.41 \mathrm{E}-0 \mathrm{O}=$ & $8.36 \mathrm{E}-08=$ & & all, No dam. & $128=$ & $9.51 E-08=$ & $6.43 \mathrm{E}-04$ & \\
\hline & all,HEPA & $56=$ & $3.74 \mathrm{E}-08=$ & $5.74 \mathrm{E}-06=$ & $2.64 \mathrm{E}-07$ & & ail,,HEPA & 58 & $3.74 E-08=$ & $8.82 E-02$ & \\
\hline & all, Dome crac & $16<$ & $3.99 E-08 \mid<$ & $3.70 \mathrm{E}-04<$ & $9.76 \mathrm{E}-05<<$ & & all,Dome crac & 16 < & $3.99 \mathrm{E}-08$ & $8.98 E-01$ & \\
\hline & all, Dome fail. & $68>$ & $4.31 E-08>$ & $3.48 E-03>$ & $6.08 \mathrm{E}-04>$ & & ail, Dome fail. & $68>$ & $4.31 \mathrm{E}-08$ & $1.83 E+01$ & \\
\hline & & & & & & & & & & & \\
\hline & & & & & & & & & & & \\
\hline
\end{tabular}




\begin{tabular}{|c|c|c|c|c|c|c|c|c|c|c|c|}
\hline \multicolumn{4}{|c|}{ Waste intrusive equipment - OnSite Rad - Consequences } & & \multirow{2}{*}{ Expected } & \multicolumn{4}{|c|}{ Waste intrusive equipment - Onsit Tox - Consequences } & \multirow{2}{*}{\multicolumn{2}{|c|}{ Meclas cons }} \\
\hline Tank & & No. events & Median treq. & Median cons. & & Tank & & INo. wants & Medlar freq. & & \\
\hline \multirow[t]{2}{*}{ S-102 } & Tail TOS & $2129=$ & $2.26 \mathrm{E}-07$ & $2.075-04$ & $1.15 E-05$ & $5=102$ & all ToS & 2129 & $2.2 B E-07$ & $\geq$ & $1.46 E+\infty]<$ \\
\hline & WIEquip (all ? & 131 & $4.47 E-03$ & $3.31 E-08$ & $3.28 E-05$ & & WIEquip (all ) & 13 & $4,47 E-03$ & 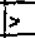 & $1.00 \mathrm{E} \cdot 01 \mathrm{2}$ \\
\hline \multirow[t]{2}{*}{$u-111$} & an TOS & 7455 & $1.34 E-67$ & $9.17 E-06$ & 8.59E-04 & Uu-119 & all TOS & 745 & $1.34 E-07$ & 2 & $9.48 E-033$ \\
\hline & WiEguip (all ? & 29 & $1.97 \mathrm{E}-02$ & $7.31 E-05$ & $3.00 E-02$ & & WiEguip (ail & $29:$ & $7.31 E-05$ & $\geq$ & $1.82 \mathrm{E}+01>$ \\
\hline \multirow[t]{2}{*}{ T-203 } & All TDS & $1621=$ & $2.14 E-08=$ & $3.13 E-04$ & $5.29 E-06$ & $T \cdot 203$ & alt TDS & 1621 & $2.14 E-08$ & $=$ & $1.34 E+00=$ \\
\hline & WiEquip \{all & $1>$ & $5.03 E-02$ & $2.02 E-03$ & $2.03 E-04$ & & WIEquip (ali 1 & $1=$ & $5.03 E-02$ & $\geq$ & $1.73 E+025$ \\
\hline \multirow[t]{3}{*}{$8-111$} & aㅐ TDS & $2721>$ & 5.92E-09: & $5.03 E-06=$ & 2.11E-06 & $0-111$ & all TDS & 2727 & $5.92 E-08$ & $\geq$ & $3.36 \mathrm{E}-02 \mathrm{P}$ \\
\hline & WIEguip (all ? & -4) & $1.29 E-01\}$ & $1.26 E-05$ & $1.03 E-05$ & & WIEquip (all ? & $4)$ & $1.29 E-01$ & $\sqrt{2}$ & $3.35 \varepsilon+\infty)>$ \\
\hline & & & & & & & & & & & \\
\hline \multicolumn{4}{|c|}{ Waste intrusive equipment - OnSite Fad - Consequences } & & Expected & Waste if & isive exuipment - & - OnSile Tox - & Sonsequances & & \\
\hline Tank & & No. events & Median treq. & Median cons. & Fisk & Tank & & No. events & Median freq. & & Median cons. \\
\hline S. 102 & all TOS & $27861>$ & $1.65 E-06$ & $1.56 \mathrm{E}-04$ & $1.37 \varepsilon-03$ & s-102 & Tall TOS & T2786 & $1.65 \mathrm{E}-00$ & $>$ & $2.79 E+00$ \\
\hline & WiEquip (all) & $670>$ & $7.68 \mathrm{E}-03$ & $1.69 \mathrm{E}-0.5$ & $3.63 E-0.9$ & & WIEquip (all 7 & 670 & $7.68 \mathrm{E}-03$ & $>$ & $1.11 \mathrm{E}+01 ?$ \\
\hline $\mathrm{U}-111$ & all TOS & $1403>$ & $4.97 \mathrm{E}-05$ & $4.41 E-08$ & $1.18 E-0.91$ & U.111 & aul TDS & 1403 & $4.97 E-05$ & $\geq$ & $5.11 E-01>$ \\
\hline & WIEquip (afr & $687>$ & $9.54 E-03 / 5$ & 1.65E-05 & $2.85 E-03$ & & WIEquip (al ? & 6875 & $9.54 \mathrm{E} .03$ & $\geq$ & $7.89 E+\infty 0<<$ \\
\hline$T-203$ & allos & 2387 ? & $8.20 \mathrm{E}-07$ & $2.09 E-04$ & $1.01 E-02$ & $T-203$ & all TDS & $2387>$ & $6.20 \mathrm{E}-07$ & $>$ & $2.60 E+00>$ \\
\hline & WiEquip (all & $767>>$ & $8.89 \mathrm{E}-03 \mathrm{~S}$ & 5.91E-05 & $2.33 E-02$ & & WIEquip \&all & $767>$ & $8.89 E-03$ & & $1.38 \mathrm{E}+01 \mathrm{~K}$ \\
\hline$z-1+1$ & ail TOS & $1038>$ & $3.44 E-0317$ & $1.46 E-05$ & $1.15 E-03$ & B-111 & all TDS & 1038 & $3.44 E-03$ & & $2.22 E+003$ \\
\hline & WiEquip (all & $770>>$ & $8.17 \mathrm{E}-0.3 \mathrm{~S}$ & $2.43 E-05$ & 2. $64 E-03$ & & WIEquip (all 1 & 770 & $8.17 \mathrm{E}-03$ & & $7.29 E+\infty)>$ \\
\hline & & 1 & & & & & 1 & & & & \\
\hline Waste if & ive equipment - & Onsite Rad-C & ingequences & & Expected & Waste in & sive equipment - & - Onsite Tox-C & insequences & & \\
\hline Tank & & No. events & Median freq. & Madian cons. & Risk & Tank & & No. events & Median freq & & Median cons, \\
\hline S-102 & all TOS & $2129=$ & $2.26 \mathrm{E}-07$ & $2.07 \mathrm{E}-04$ & $1.14 E-05$ & $s-102$ & ali TOS & $2129=$ & $|\quad 2.26 E-07|$ & $\approx$ & $1.46 E+\infty 0=$ \\
\hline & WIEquip (all 7 & $13=$ & $4.47 \mathrm{E} \cdot 03=$ & $2.31 E-08.5$ & $2.27 \mathrm{E}-05$ & & WIEquip (all 7 & $13=$ & $4.47 E-03$. & 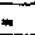 & $7.61 \mathrm{E}-02<<$ \\
\hline$u-111$ & Tal Tos & $745=$ & $1.34 \mathrm{E}-07=$ & $1.17 E-06=$ & $6.07 E-04$ & $U-111$ & all TOS & 745 is & $1.34 E-07=$ & $=$ & $9.48 E-03=$ \\
\hline & WiEquip (all I) & $29=$ & $1.97 E-02=$ & $5.11 \mathrm{E}-05 \mathrm{~K}$ & $2.09 E-02$ & & WIEquip (all 7 & $29=$ & 1.97E-02: & $>$ & $1.27 \mathrm{E}+01<\mathrm{C}$ \\
\hline $\mathrm{T}-203$ & all TOS & $1621=$ & $2.14 E-08$ & $3.13 \mathrm{E}-\mathrm{C} 4=$ & $5.23 E-08$ & $T-203$ & aallTOS & $1621=$ & $2.14 E-08$ & $\equiv$ & $1.34 \mathrm{E}+00=$ \\
\hline & | WIEquip (anl 7 & $1=$ & $5.03 \mathrm{E}-02=$ & $1.42 E-03<\mathrm{C}$ & $1.43 E-04$ & & WIEquip (all 7 & $1 / x$ & $5.03 E-02$ & \pm & $0.21 E+0313$ \\
\hline B. +111 & allitos & $272=$ & $5.92 E-08=$ & $5.03 E \cdot 06=$ & $2.10 E-06$ & $\mathrm{~B}-111$ & all TOS & $272=$ & $5.92 E-08$ & & 3.36E-02 $=$ \\
\hline & WIEquip (all? & $4=$ & $1.29 \mathrm{E}-01=$ & $8.84 \mathrm{E}-06<$ & $7.23 E-08$ & & WIEquip (all t] & $4=$ & $1.29 \mathrm{E}-01$ & $=$ & $2.36 \mathrm{E}+00<$ \\
\hline & 1 & & & & & & & & & & \\
\hline Waste in & ive equipment - & - OnSite Rad-Co & onsequences & & Expected & Waste in & ive equipment- & - OnSite Tox - co & onsequances & & \\
\hline Tank & & No. events & Median treq. I & Medlan cons & \begin{tabular}{|l|l|} 
Fisk & \\
\end{tabular} & Trank & $T$ & No, events & Median freq. & & Madian cons. \\
\hline $5-102$ & aid TDS & $2129=$ & $2.26 \mathrm{E}-07$ & $2.07 E-0 \mathrm{a}=$ & $1.15 E-05$ & $8-102$ & all Tos & $2129=$ & $2.26 \mathrm{E}-07$ & 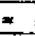 & $1.46 E+\infty=$ \\
\hline & wiEquip (all 7 & $13=$ & $4.47 \mathrm{E}-03=$ & $2.98 \mathrm{E}-0 \mathrm{~B}<\mathrm{S}$ & $2.94 E-05$ & & WiEquip (atl 7 & 13 & $4.47 E-03=$ & & $9.82 \mathrm{E}-02<\mathrm{C}$ \\
\hline$U-111$ & all TDS & $745=$ & $1.34 \mathrm{E}-0 \mathrm{r}=$ & $1.17 E-06$ & $7.61 E-045$ & $u-11 ?$ & all TOS & $745=$ & $7.34 E-07)$ & $=$ & $9.48 E-09)=$ \\
\hline & WiE quip (ant & $29=$ & $1.97 E-02=$ & $5.11 \mathrm{E}-05 \mathrm{~K}$ & $2.65 E-02$. & & WIEquip fail 1 & $29=$ & $\mid 1.97 \mathrm{E}-02$ & & $1.61 \mathrm{E}+01 \mathrm{~K}$ \\
\hline$T-203$ & all TDS & $+621=$ & $2.14 \mathrm{E}-00 \mathrm{O}=$ & 3.13E-04 = & $5.32 E-06=$ & $T-203$ & all TOS & $1621=$ & $2.14 \mathrm{E}-0 \mathrm{0}$ & & $1.34 E+00=$ \\
\hline & WIEquip (al) & $1=$ & $5.03 \mathrm{E}-02 . \mathrm{x}$ & $2.37 E-03>$ & $2.386-04$ & & WIEquip lail & 1 & $5.03 E-02=$ & & $2.01 E+037>$ \\
\hline B-111 & all Tos & $272=$ & $5.92 \varepsilon-08=$ & $5.03 E-06=$ & $2.11 E-06$ & B-111 & all TDS & $272=$ & $5.92 \mathrm{E}-08=$ & & $3.36 E-02=$ \\
\hline & WIEquip (all T] & 4 & $1.29 \mathrm{E}-01 \mathrm{I}$ & $1.12 E-05<$ & $9.15 E-06<5$ & & wiEquip (all) & $4=$ & $1.29 \mathrm{E}-01$ & & $2.98 E+00 \mid<$ \\
\hline & & & & $\therefore \quad 1$ & & & & & & & \\
\hline & & & & & & & & & & & \\
\hline & & & & & & & & & & & \\
\hline & & & & & & & & & & & \\
\hline & & & & & & & & & & & \\
\hline & & & & & & & & & & & \\
\hline & & & & & & & & & & & \\
\hline & & & & & & & & & & & \\
\hline & & & & & & & & & & & \\
\hline & & & & & & & & & & & \\
\hline & & & & & & & & & & & \\
\hline & & & & & & & & & & & \\
\hline & & & & & & & & & & & \\
\hline & & & & & & & & & & & \\
\hline & & & & & & & & & & & \\
\hline & & & & & & & & & & & \\
\hline & & & & & & & & & & & \\
\hline & & & & & & & & & & & \\
\hline & & & & & & & & & & & \\
\hline & & & & & & & & & & & \\
\hline & & & & & & & & & & & \\
\hline & & & & & & & & & & & 1 \\
\hline & & & & & & & & & & & 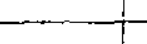 \\
\hline & & & & & & & & & & & \\
\hline & & & & & & & & & & & \\
\hline
\end{tabular}


RPP-6888, Rev. 0

This page intentionally left blank 
Percent

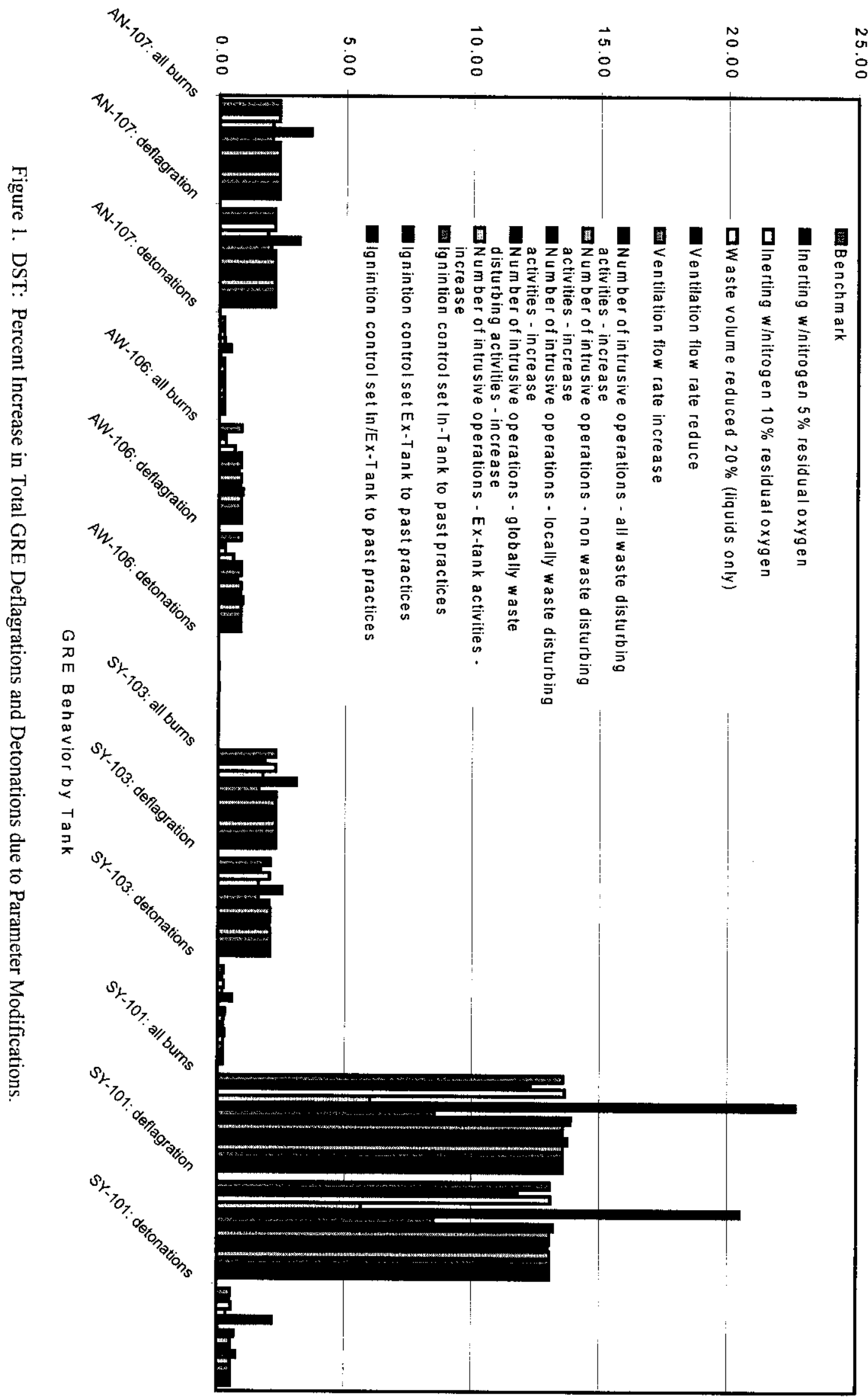

0
0
0
0
3
0
0
-1
0
0
0
10
0
0
2
0
3
3
0
0
0
0
0
0 


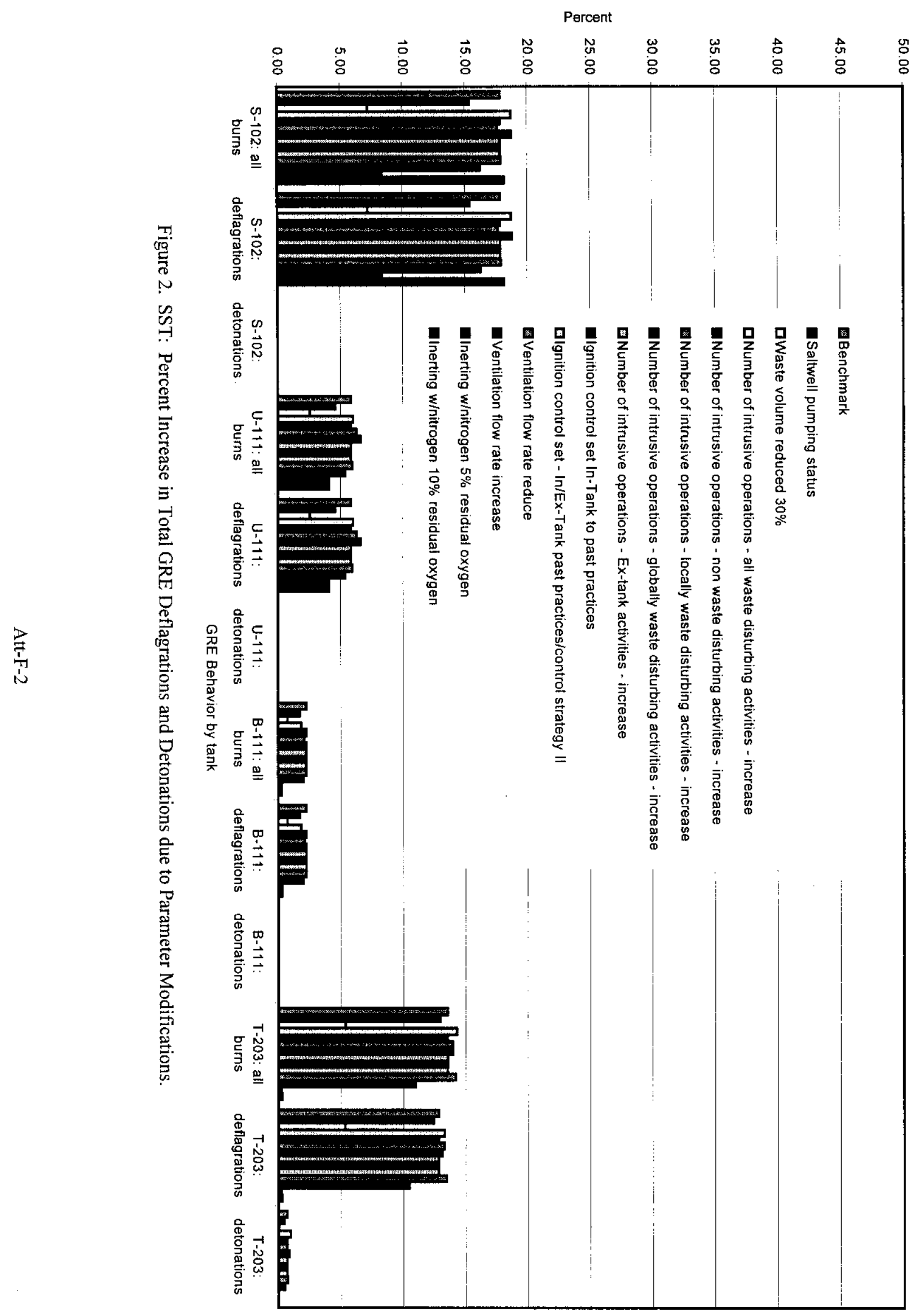

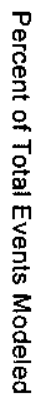




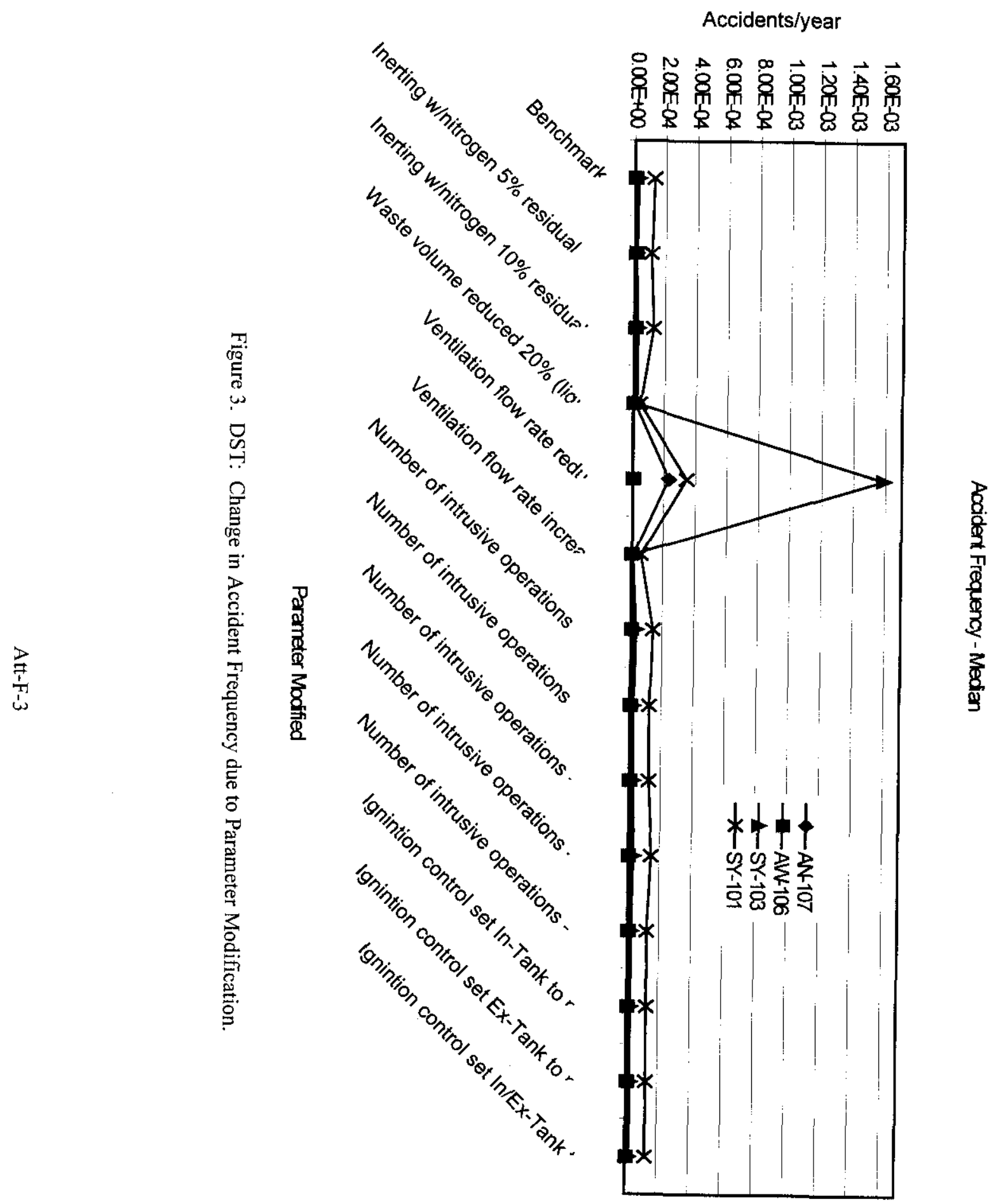

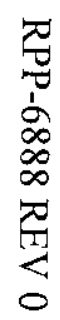




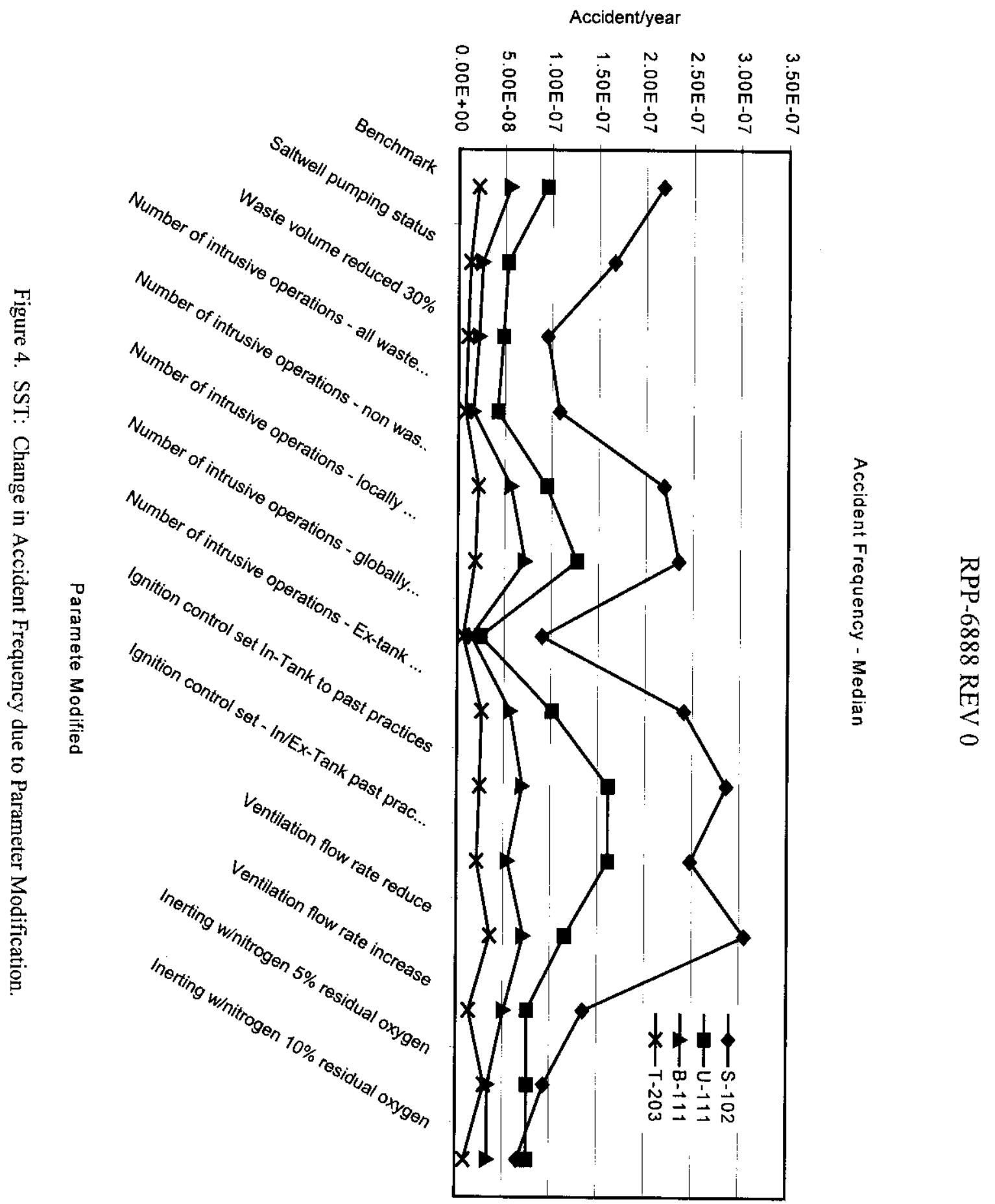




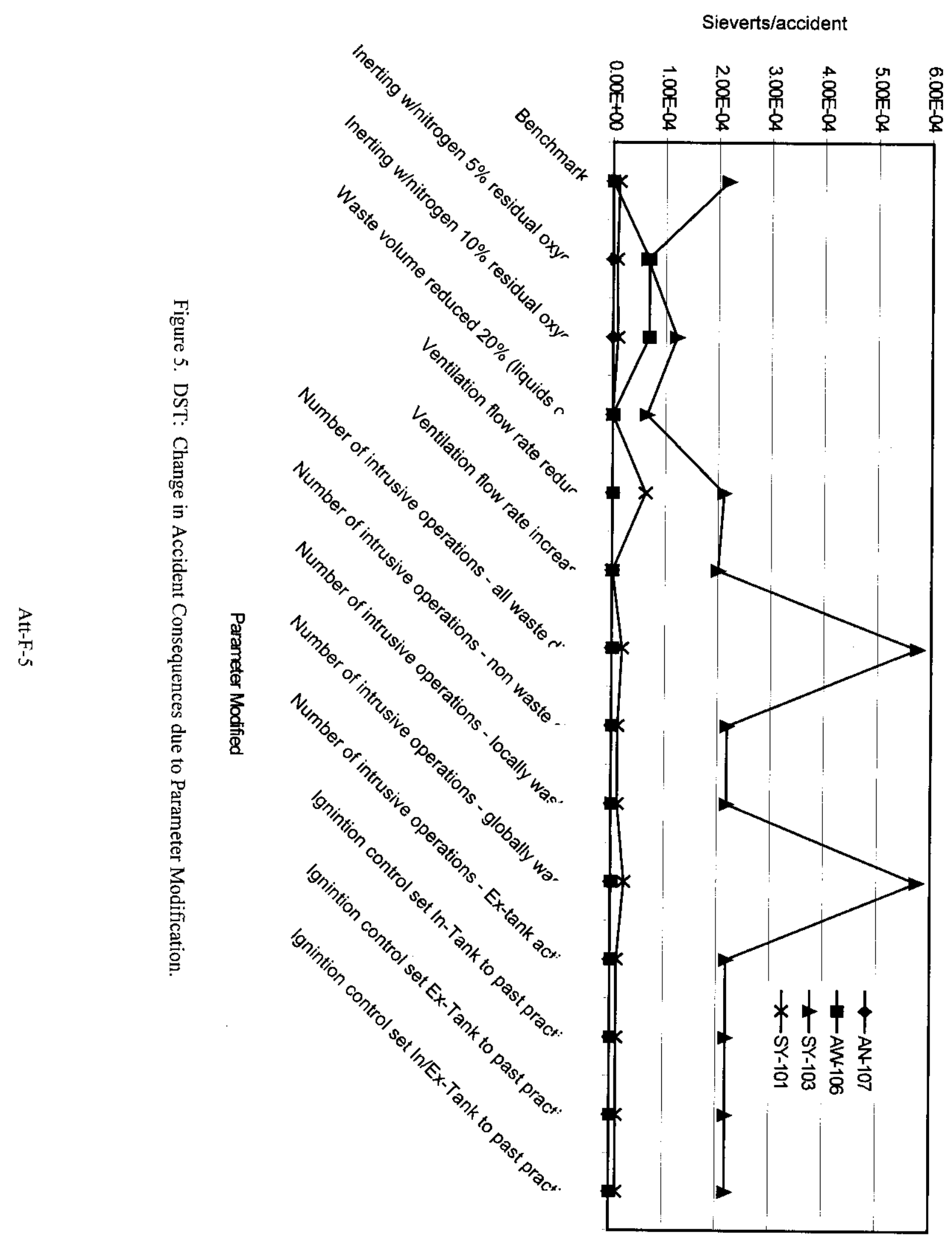

8
$\frac{8}{8}$
$\frac{3}{8}$
8
$\frac{8}{8}$
$\frac{5}{5}$
$\frac{8}{8}$
$\frac{1}{8}$
$\frac{8}{9}$ 
$\frac{2}{\frac{1}{1}}$

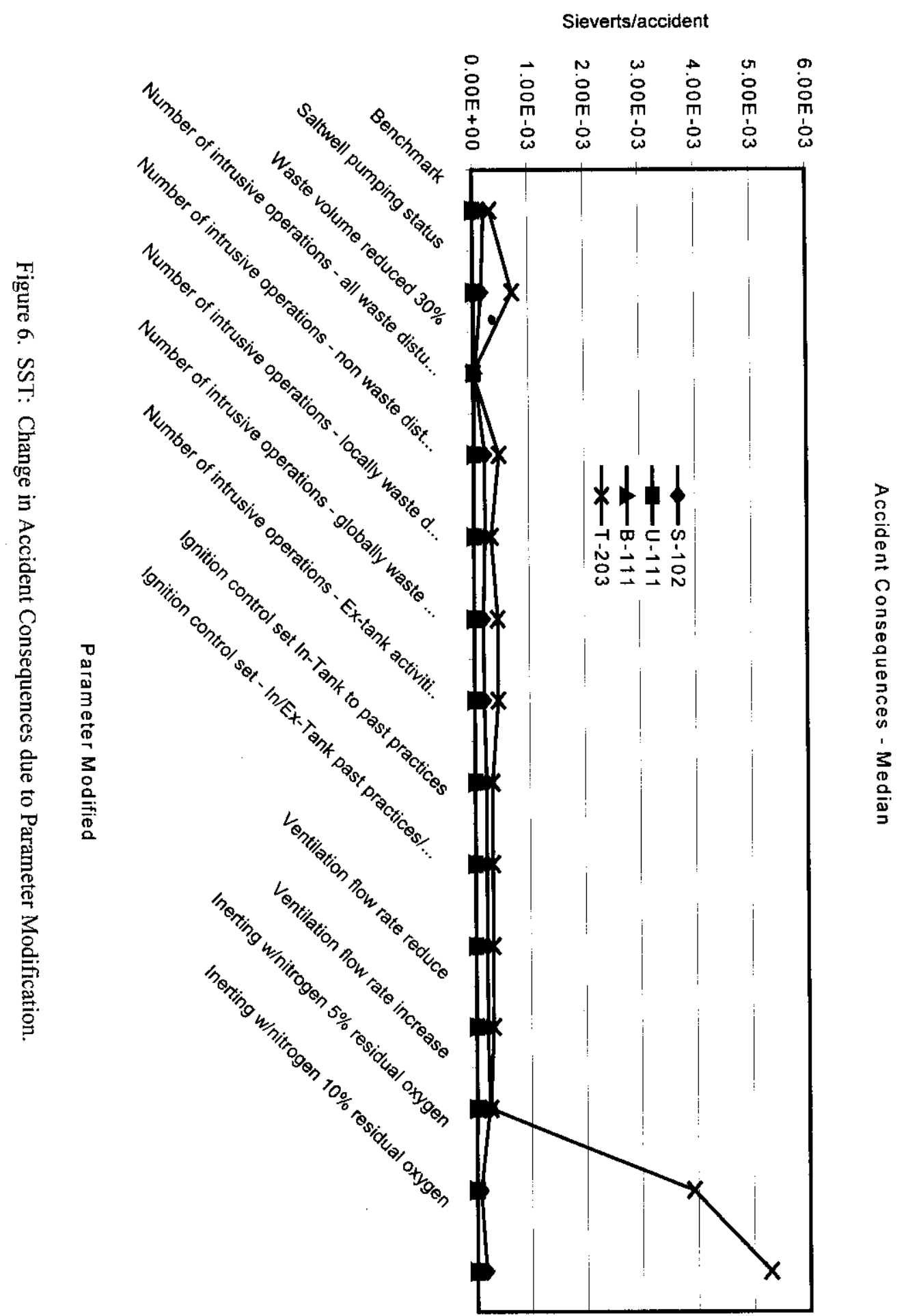




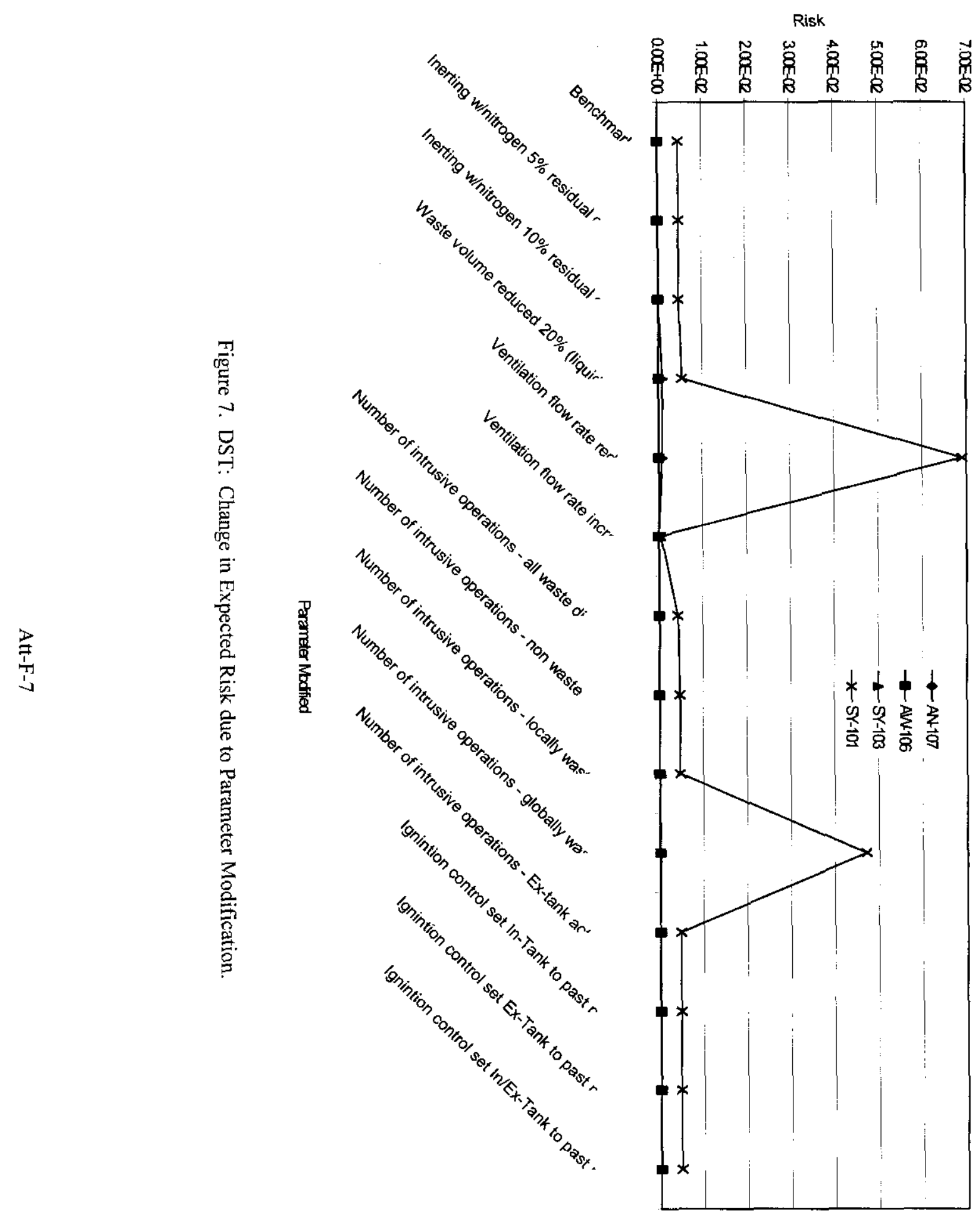

2
0
0
$\infty$
$\infty$
$\infty$
2
0
0
0 







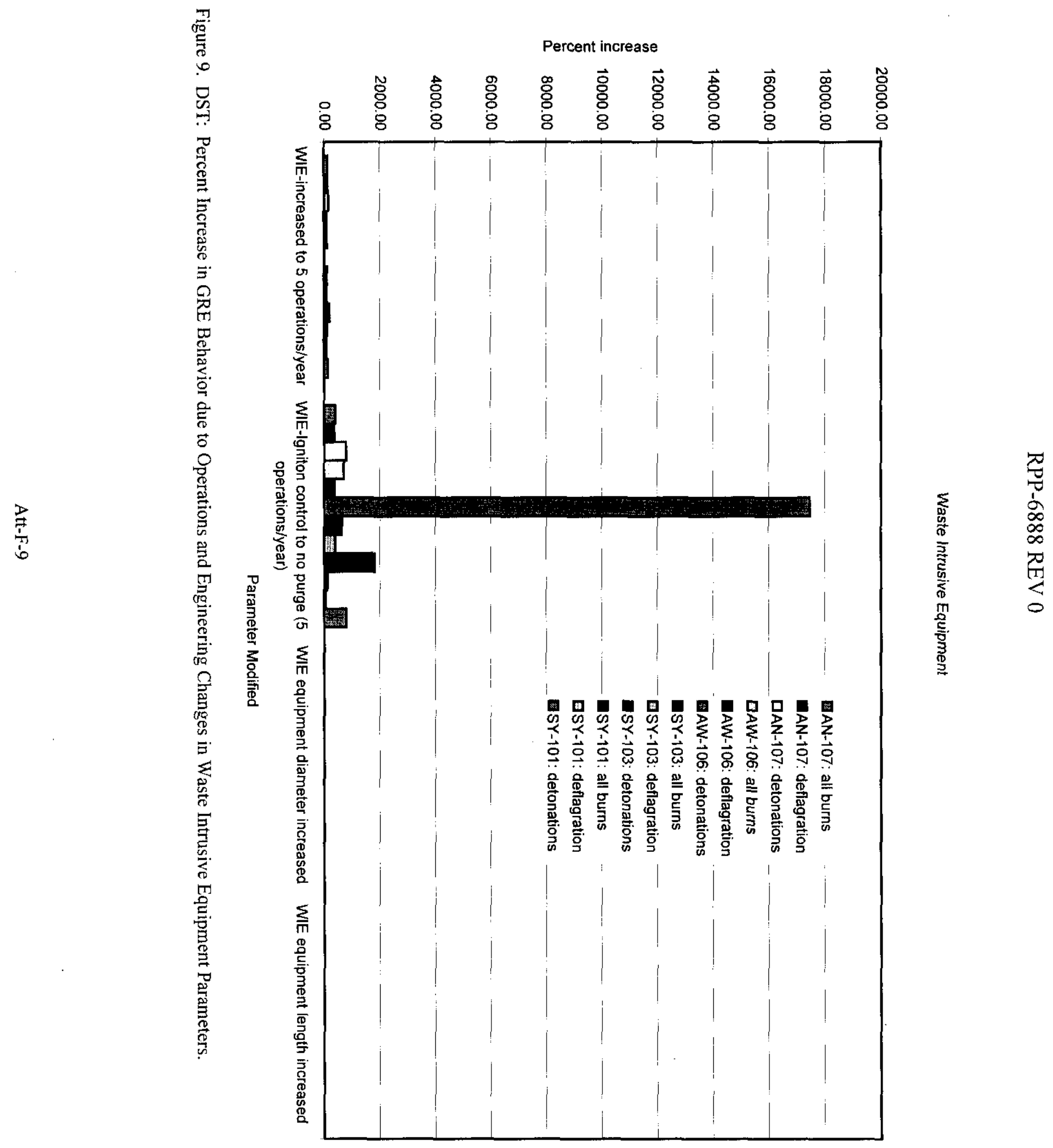




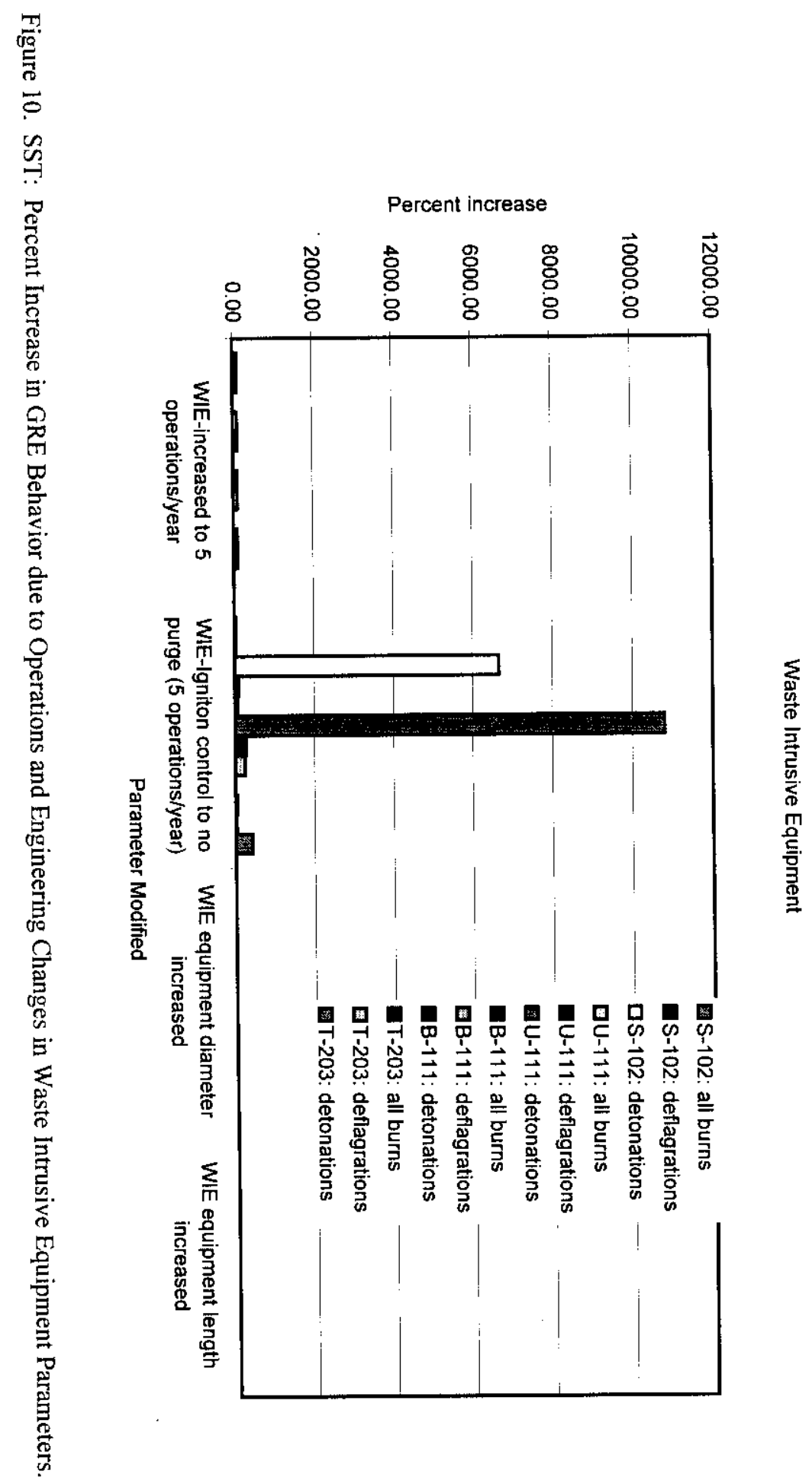




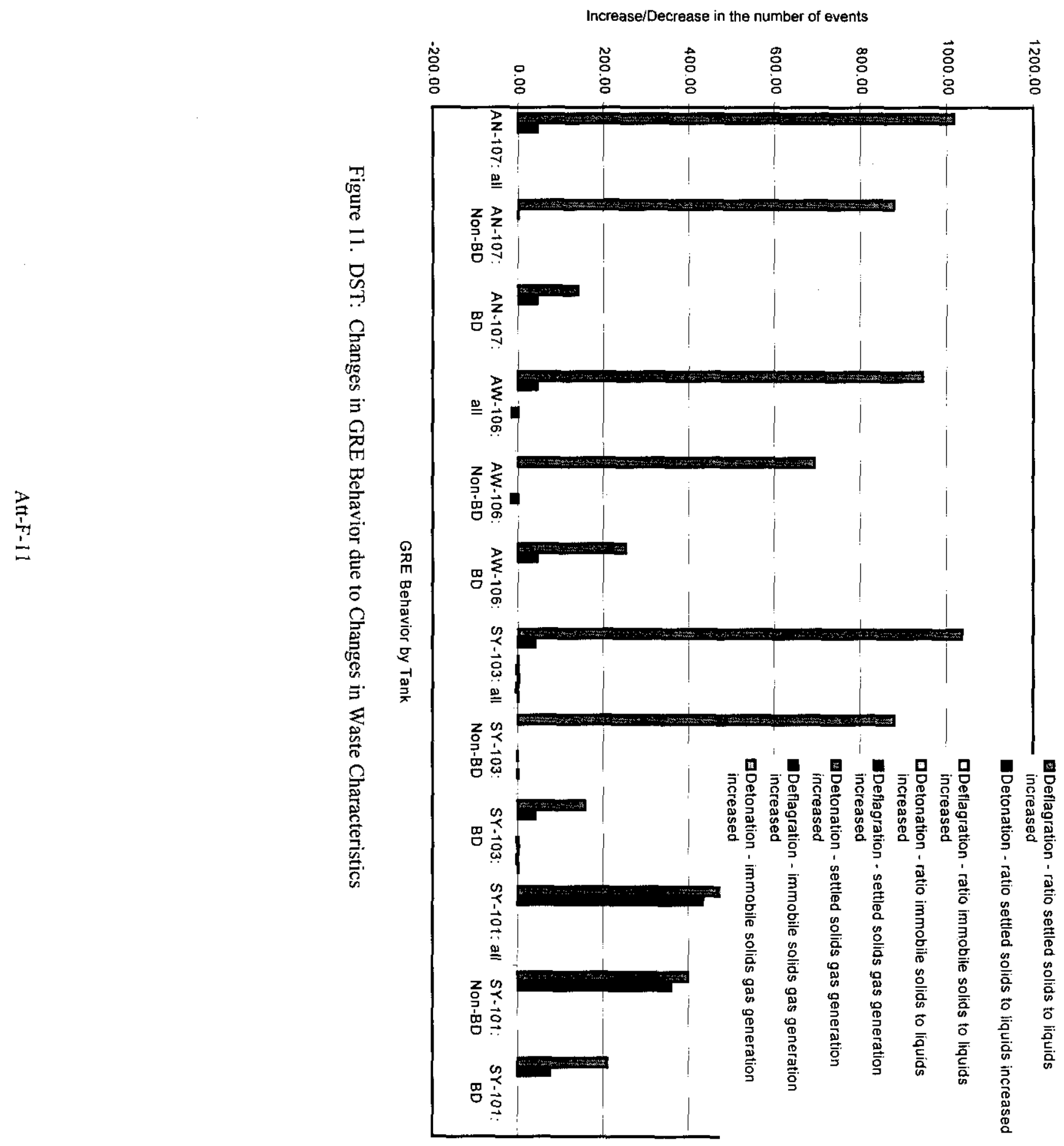




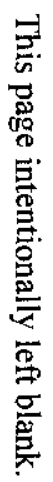

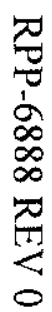

